\title{
ELECTROKINETIC METHODS FOR PREPARATIVE ELECTROPHORESIS ON A CHIP
}

Dawid R. Zalewski 
The research described in this thesis was carried out at the Mesoscale Chemical Systems group and the Biochip group of the MESA+ Institute for Nanotechnology at the University of Twente. The project was partially supported by The Netherlands Organisation for Scientific Research - NWO.

Graduation committee:

\section{Chairman}

Prof. dr. Gerard van der Steenhoven Universiteit Twente Secretary

Prof. dr. Gerard van der Steenhoven Universiteit Twente

\section{Promotor}

Prof. dr. Han J.G.E. Gardeniers

Universiteit Twente

Assistant promotor

Dr. ir. Richard B.M. Schasfoort

Universiteit Twente

Members

Prof. dr. Vinod Subramaniam

Universiteit Twente

Dr. Jan C.T. Eijkel

Universiteit Twente

Prof. dr. ir. Gert Desmet

Vrije Universiteit Brussel

Prof. dr. Thomas Hankemeier

Universiteit Leiden

Prof. dr. ir. Jaap M.J. den Toonder

Technische Universiteit Eindhoven

Electrokinetic Methods for Preparative Electrophoresis on a ChipDawid R. Zalewski

PhD thesis, University of Twente, Enschede, The Netherlands

ISBN: 978-90-365-2722-4

Copyright (C) 2008 by Dawid R. Zalewski, Enschede, The Netherlands 


\title{
ELECTROKINETIC METHODS \\ FOR PREPARATIVE ELECTROPHORESIS ON A CHIP
}

\section{DISSERTATION}

\author{
to obtain
}

the degree of doctor at the University of Twente, on the authority of the rector magnificus,

$$
\text { prof. dr. W. H. M. Zijm, }
$$

on account of the decision of the graduation committee, to be publicly defended on Friday, the $24^{\text {th }}$ of October 2008 at 15.00 hrs

$$
\text { by }
$$

\section{Dawid Radosław Zalewski}

born on the $11^{\text {th }}$ of January 1979

$$
\text { in Sulęcin, Poland }
$$


This dissertation has been approved by:

Prof. dr. Han J. G. E. Gardeniers (promotor)

Dr. Richard B. M. Schasfoort (assistant promotor) 



\section{Table of Contents}

\section{ChAPTER I}

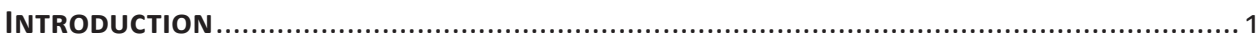

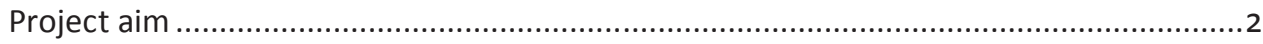

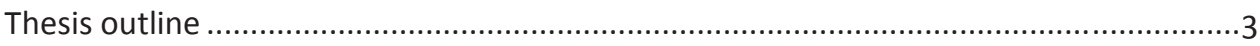

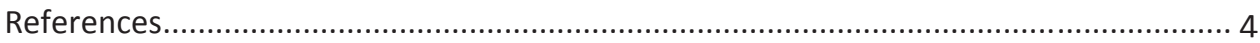

\section{CHAPTER II}

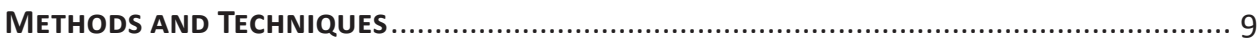

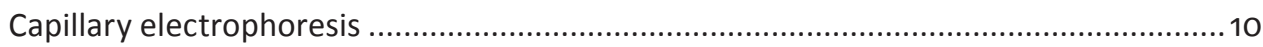

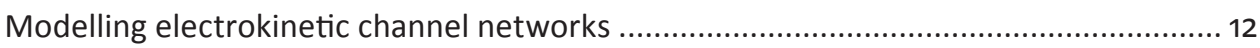

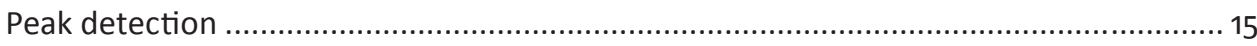

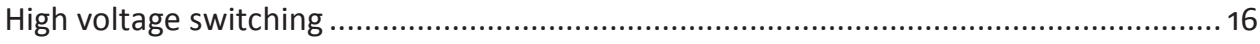

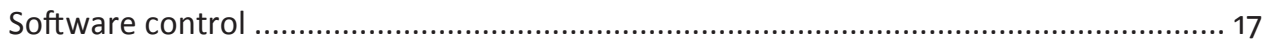

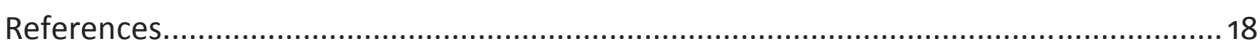

\section{CHAPTER III}

Electrokinetic Manipulation of CE Separated Fractions

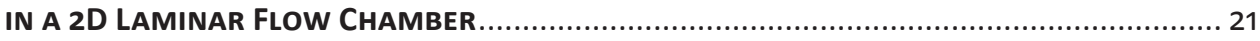

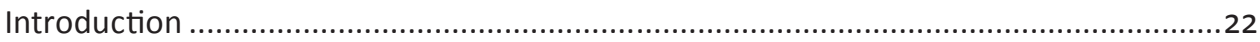

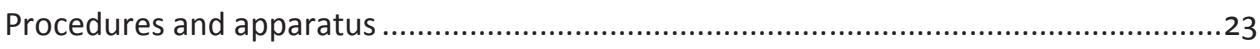

Chemicals $\cdot 23 \mid$ Chip fabrication $\cdot 24 \mid$ Setup and chip operation $\cdot 24$

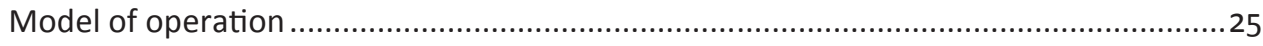

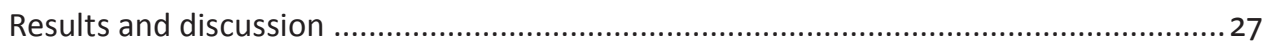

Sample stream positioning $\cdot 27 \mid$ Manipulation of separated fractions $\cdot 30$

Performance with high sample load · 31 | Manipulation with sample recirculation $\cdot 33$ | Conclusions $\cdot 34$

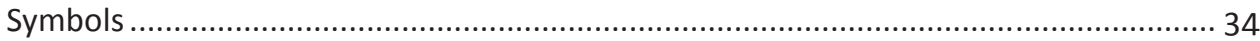

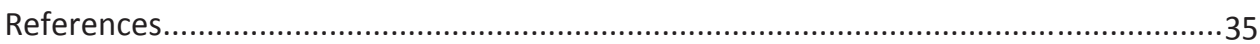

CHAPTER IV

FORCED SPLITTING OF Fractions In CAPILLARY EleCtrophoresis ...............................39

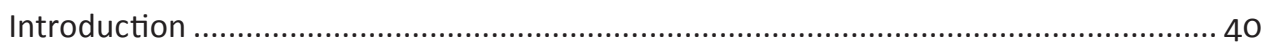

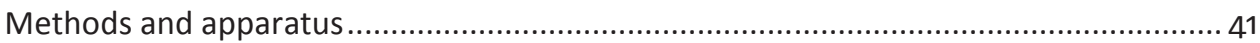

Principle of forced splitting $\cdot 41 \mid$ Materials $\cdot 42$ | Device fabrication $\cdot 42$

Instrumentation and modelling $\cdot 42$

Results and discussion

Preparative CE chip · 43 | Optimal splitting conditions · 44 | Experimental validation of forced splitting $\cdot 47 \mid$ Conclusions $\cdot 52$

References. 


\section{ChAPTER V}

ELECTROKINETIC SORTING AND COLLeCtion OF FRACTIONS

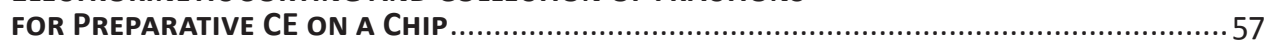

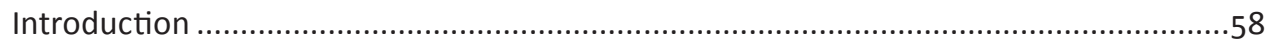

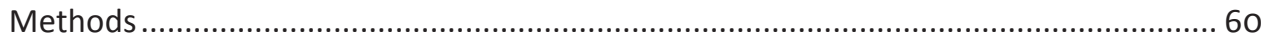

General system considerations $\cdot 61 \mid$ Model of operation $\cdot 63$

Performance measures $\cdot 65$

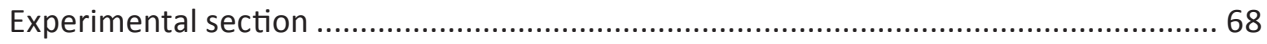

Materials $\cdot 68 \mid$ Device fabrication $\cdot 69 \mid$ Instrumentation $\cdot 70$

Chip operation $\cdot 70$

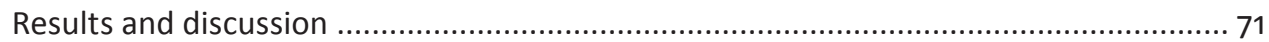

Fraction collection and accumulation $\cdot 71 \mid$ Overlapping fractions $\cdot 73$

Time stability and repeatability $\cdot 75 \mid$ Conclusions $\cdot 75$

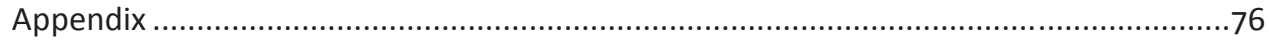

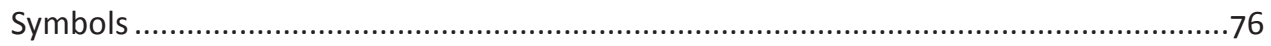

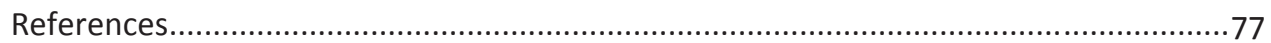

CHAPTER VI

SYNCHRONIZED, CONTINUOUS-FLOW ZONE ELECTROPHORESIS .................................. 83

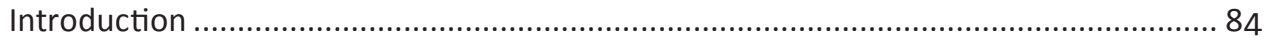

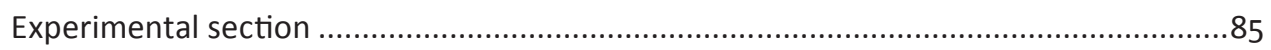

Theory of operation $\cdot 85 \mid$ Synchronized steering $\cdot 87 \mid$ Microchip

fabrication $\cdot 88 \mid$ Chemicals $\cdot 88 \mid$ Apparatus and procedures $\cdot 88$

Results and discussion

Chip devices $\cdot 89 \mid$ Separation $\cdot 90 \mid$ Synchronized collection

and purification $\cdot 92$ | Contamination $\cdot 94 \mid$ Conclusions $\cdot 98$

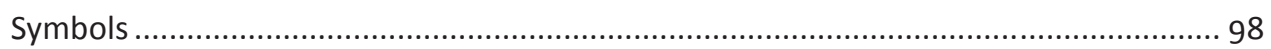

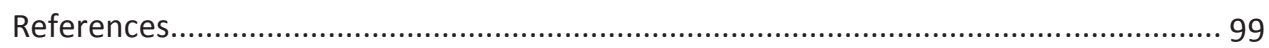

ChAPTER VII

Fractionation Of A TWo-CoMponent MixtURE by SCFZE ................................... 103

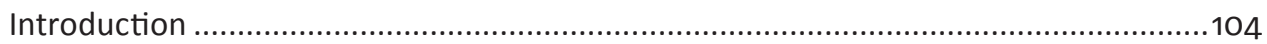

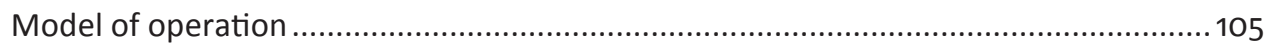

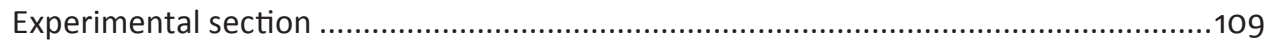

Microchip fabrication $\cdot 109 \mid$ Chemicals and procedures $\cdot 109$

Results and discussion .110

Chip device $\cdot 110 \mid$ Sample fractionation $\cdot 111 \mid$ CE of fractionated sample $\cdot 116$

Performance measures of fractionation $\cdot 117 \mid$ Conclusions $\cdot 119$ 
Symbols

References.

\section{ChAPTER VIII}

CONCLUSIONS AND OUTLOOK

Hardware performance. 124

Junctions optimization.... 124

Electric field in SCFZE .... 126

Outlook for SCFZE 130

References. 130

APPendix A 133

APPENDIX B

File 'SCFZE.m' 133

\section{APPENDIX C}

File 'DSCFZE.m' $139 \mid$ File 'TemporarySaveBeginWrite.m' 146

File 'SaveDataHelper.m' 147

SUMMARY

SAMENVATTING .151

ACKNOWLEDGEMENTS 155 



\section{Chapter I Introduction}

His CHAPTER PRESEnts the objectives of the project as
well as background information on prior developments of capillary electrophoresis with special emphasis on preparative techniques. 


\section{Project aim}

The concept of capillary electrophoresis was introduced by Hjerten in 1967. ${ }^{1}$ However, the idea had not been further researched until late 1970s when Mikkers ${ }^{2}$ and later Jorgenson ${ }^{3,4}$ presented their experiments on free zone electrophoresis in narrow tubes. Nowadays, capillary electrophoresis is an established analytical technique in biological sciences. ${ }^{5-11}$ Certainly, its development was greatly accelerated during the Human Genome Project. ${ }^{12}$ Upon sequencing of the human DNA $^{13,14}$ CE instruments became standard laboratory equipment. Nowadays it used not only for DNA fragment analysis, but also for e.g. proteins and metabolites, and in clinical and forensic applications.

The first microfluidic system integrated on a chip device was presented by Manz in $1990 .{ }^{15}$ Its demonstration was preceded - several pages earlier in the same journal - by the introduction of the term miniaturized total chemical analysis system ${ }^{16}$ ( $\mu$-TAS, nowadays: micro total analysis system). The idea of combining multiple analytical techniques in a single microdevice attracted considerable attention and soon many such systems appeared. ${ }^{17-21}$ Among them was a miniaturized capillary electrophoresis device shown in 1992 by Harrison and Manz. ${ }^{22,}{ }^{23}$ Since the pioneering demonstration, microchip capillary electrophoresis has grown to become an important branch of analytical sciences ${ }^{24,25}$ and a dynamic increase in the number of publications targeting its development and applications has been observed in the recent years. ${ }^{26}$

The main purpose of capillary electrophoresis has always been analysis and thus most of the research effort is targeted towards improvement of its analytical powers. Yet, the attractiveness of the approach triggered the development of preparative methods utilizing CE. The first demonstration of micropreparative CE by Hjerten and Zhu was done in $1985 .{ }^{27}$ The manual switching of a capillary outlet between collection tubes was later investigated by others. ${ }^{28,29}$ Rose and Jorgenson introduced some level of automation into the collection process ${ }^{30}$ and after years of development a fully-automated preparative CE apparatus was described by Muller ${ }^{31}$ - the idea was brought to even higher sophistication level by Irie et al. in $2000 .^{32}$

Three years after the demonstration of a microchip CE device Effenhauser showed the possibility of manipulating separated fractions in a simple microfluidic network. ${ }^{33}$ Soon, other reports were published showing different methods of performing nearly-preparative CE. ${ }^{34-36}$ Nearly, because in all designs retrieval and 
pooling of only one fraction at once was possible. Also, only the device presented by Tullock ${ }^{36}$ utilized an automated collection procedure.

There are many causes for the poor development of preparative CE techniques. Hempe states that CE methods are essentially nonpreparative and fraction collection remains technically challenging. ${ }^{37} \mathrm{He}$ also identifies the main problem that constitutes this statement: very limited amount of sample material per separation. Consequently he writes: Peaks from multiple CE runs can be pooled to increase sample recovery but consistent fraction collection requires highly reproducible run to run separations and accurate prediction of post-detector elution from the capillary. ${ }^{37}$

In this study, the aim is the development of microfluidic electrokinetic-only strategies for microchip preparative CE. Particularly, the techniques for single fraction manipulation in planar chambers, as well as in complex channel networks are researched and methods that allow for fully automated control of such procedures are presented. Furthermore, an approach for continuously operating, preparative zone electrophoresis is introduced and investigated.

\section{Thesis outline}

\section{Chapter 2}

In this chapter, the theoretical background of capillary electrophoresis and electrokinetic flow control in channel networks is introduced. Emphasis is put on a general understanding of the concepts used later in the thesis. Also a description of the commonly used tools is given.

\section{Chapter 3}

Electrokinetic transport of separated fractions is usually achieved in channel networks incorporating many junctions. In this chapter an alternative way is described. A microfluidic planar chamber is used instead and paths of the fractions are controlled by fast electrokinetic flow switching. A theoretical description is followed by an experimental validation of the device functioning as a preparative tool.

\section{Chapter 4}

The miniaturization of CE brings one important drawback: the resolution of separation is very limited as compared to traditional instruments. Consequently, separated fractions often overlap or are closely spaced at the end of a separation 
channel, making precise handling of individual peaks virtually impossible. In this chapter a method for forced electrokinetic splitting of adjacent fractions is proposed, which can be straightforwardly integrated into a micropreparative CE chip design.

\section{Chapter 5}

A micropreparative capillary electrophoresis chip is described in this chapter. The principle of operation is based on the splitting principle described in chapter 4 . The device is operated automatically - that is with no user interaction during operation. It allows for identical fractions pooling and discarding of undesired peaks. Also the theoretical limits of such an approach are given.

\section{Chapter 6}

A method of performing preparative CE in a continuous-flow device is introduced. The system is controlled electrokinetically and allows for zone electrophoretic fractionation of a complex mixture. Pooling and recovery of one fraction is possible during a single run. A detailed theoretical description of the method is provided together with a discussion on fractionation limits.

\section{Chapter 7}

This chapter describes a follow-up development of the method described in chapter 6. Two fractions can be simultaneously pooled during a single run. Moreover, nearly contamination-free fractionation is achievable with proper operating parameters.

\section{Chapter 8}

Micropreparative CE techniques require further development. In this chapter some critical aspects that need to be addressed are identified together with potential solutions.

\section{References}

1. Hjertén, S., Free zone electrophoresis. Chromatographic Reviews, 1967. 9(2): p. 122-219.

2. Mikkers, F.E.P., F.M. Everaerts, and T. Verheggen, High-Performance Zone Electrophoresis. Journal of Chromatography, 1979. 169(FEB): p. 11-20.

3. Jorgenson, J.W. and K.D. Lukacs, Zone Electrophoresis in Open-Tubular GlassCapillaries. Analytical Chemistry, 1981. 53(8): p. 1298-1302. 
4. Jorgenson, J.W. and K.D. Lukacs, Free-zone electrophoresis in glass capillaries. Clinical Chemistry, 1981. 27(9): p. 1551-3.

5. Huang, Y.F., C.C. Huang, C.C. Hu, and H.T. Chang, Capillary electrophoresis-based separation techniques for the analysis of proteins. Electrophoresis, 2006. 27(18): p. 3503-22.

6. Kraly, J., M.A. Fazal, R.M. Schoenherr, R. Bonn, M.M. Harwood, E. Turner, M. Jones, and N.J. Dovichi, Bioanalytical applications of capillary electrophoresis. Analytical Chemistry, 2006. 78(12): p. 4097-4110.

7. Dolnik, V., Capillary electrophoresis of proteins 2003-2005. Electrophoresis, 2006. 27(1): p. 126-141.

8. Huck, C.W., R. Bakry, L.A. Huber, and G.K. Bonn, Progress in capillary electrophoresis coupled to matrix-assisted laser desorption/ionization - time of flight mass spectrometry. Electrophoresis, 2006. 27(11): p. 2063-2074.

9. Kasicka, V., Recent developments in capillary electrophoresis and capillary electrochromatography of peptides. Electrophoresis, 2006. 27(1): p. 142-175.

10. Klampfl, C.W., Recent advances in the application of capillary electrophoresis with mass spectrometric detection. Electrophoresis, 2006. 27(1): p. 3-34.

11. Kostal, V., J. Katzenmeyer, and E.A. Arriaga, Capillary Electrophoresis in Bioanalysis. Analytical Chemistry, 2008.

12. Dovichi, N.J. and J.Z. Zhang, How capillary electrophoresis sequenced the human genome. Angewandte Chemie-International Edition, 2000. 39(24): p. 4463-4468.

13. International Human Genome Sequencing Consortium, I.H.G.S., Finishing the euchromatic sequence of the human genome. Nature, 2004. 431(7011): p. 931-945.

14. International Human Genome Sequencing Consortium, I.H.G.S., Initial sequencing and analysis of the human genome. Nature, 2001. 409(6822): p. 860-921.

15. Manz, A., Y. Miyahara, J. Miura, Y. Watanabe, H. Miyagi, and K. Sato, Design of an Open-Tubular Column Liquid Chromatograph Using Silicon Chip Technology. Sensors and Actuators B, 1990. 1(1-6): p. 249-255.

16. Manz, A., N. Graber, and H.M. Widmer, Miniaturized Total Chemical-Analysis Systems - a Novel Concept for Chemical Sensing. Sensors and Actuators B, 1990. 1(1-6): p. 244-248.

17. Reyes, D.R., D. Iossifidis, P.A. Auroux, and A. Manz, Micro total analysis systems. 1. Introduction, theory, and technology. Analytical Chemistry, 2002. 74(12): p. 2623-2636. 
18. Auroux, P.A., D. Iossifidis, D.R. Reyes, and A. Manz, Micro total analysis systems. 2. Analytical standard operations and applications. Analytical Chemistry, 2002. 74(12): p. 2637-2652.

19. Vilkner, T., D. Janasek, and A. Manz, Micro total analysis systems. Recent developments. Analytical Chemistry, 2004. 76(12): p. 3373-3385.

20. Dittrich, P.S., K. Tachikawa, and A. Manz, Micro total analysis systems. Latest advancements and trends. Analytical Chemistry, 2006. 78(12): p. 3887-3907.

21. West, J., M. Becker, S. Tombrink, and A. Manz, Micro Total Analysis Systems: Latest Achievements. Analytical Chemistry, 2008.

22. Harrison, D.J., A. Manz, Z.H. Fan, H. Ludi, and H.M. Widmer, Capillary Electrophoresis and Sample Injection Systems Integrated on a Planar Glass Chip. Analytical Chemistry, 1992. 64(17): p. 1926-1932.

23. Manz, A., D.J. Harrison, E.M.J. Verpoorte, J.C. Fettinger, A. Paulus, H. Ludi, and H.M. Widmer, Planar Chips Technology for Miniaturization and Integration of Separation Techniques into Monitoring Systems - Capillary Electrophoresis on a Chip. Journal of Chromatography A, 1992. 593(1-2): p. 253-258.

24. Dolnik, V. and S. Liu, Applications of capillary electrophoresis on microchip. Journal of Separation Science, 2005. 28(15): p. 1994-2009.

25. Peng, Y.Y., A. Pallandre, N.T. Tran, and M. Taverna, Recent innovations in protein separation on microchips by electrophoretic methods. Electrophoresis, 2008. 29(1): p. 157-178.

26. Handbook of Capillary and Microchip Electrophoresis and Associated Microtechniques. 3 ed, ed. J.P. Landers. 2007.

27. Hjerten, S. and M.-D. Zhu, Micropreparative version of high-performance electrophoresis : The electrophoretic counterpart of narrow-bore high-performance liquid chromatography. Journal of Chromatography A, 1985. 327: p. 157-164.

28. Cohen, A.S., D.R. Najarian, A. Paulus, A. Guttman, J.A. Smith, and B.L. Karger, Rapid Separation and Purification of Oligonucleotides by High-Performance Capillary Gel Electrophoresis. Proceedings of the National Academy of Sciences of the United States of America, 1988. 85(24): p. 9660-9663.

29. Guttman,A., A.S. Cohen, D.N. Heiger, and B.L. Karger, Analytical and Micropreparative Ultrahigh Resolution of Oligonucleotides by Polyacrylamide-Gel High-Performance Capillary Electrophoresis. Analytical Chemistry, 1990. 62(2): p. 137-141.

30. Rose, D.J. and J.W. Jorgenson, Fraction collector for capillary zone electrophoresis. Journal of Chromatography A, 1988. 438: p. 23-34. 
31. Muller, O., F. Foret, and B.L. Karger, Design of a High-Precision Fraction Collector for Capillary Electrophoresis. Analytical Chemistry, 1995. 67(17): p. 2974-2980.

32. Irie, T., T. Oshida, H. Hasegawa, Y. Matsuoka, T. Li, Y. Oya, T. Tanaka, G. Tsujimoto, and H. Kambara, Automated DNA fragment collection by capillary array gel electrophoresis in search of differentially expressed genes. Electrophoresis, 2000. 21(2): p. 367-374.

33. Effenhauser, C.S., A. Manz, and H.M. Widmer, Manipulation of Sample Fractions on a Capillary Electrophoresis Chip. Analytical Chemistry, 1995. 67(13): p. 2284-2287.

34. Khandurina, J., T. Chovan, and A. Guttman, Micropreparative fraction collection in microfluidic devices. Analytical Chemistry, 2002. 74(7): p. 1737-1740.

35. Lin, R., D.T. Burke, and M.A. Burns, Selective extraction of size-fractioned DNA samples in microfabricated electrophoresis devices. Journal of Chromatography A, 2003. 1010(2): p. 255-268.

36. Tulock, J.J., M.A. Shannon, P.W. Bohn, and J.V. Sweedler, Microfluidic separation and gateable fraction collection for mass-limited samples. Analytical Chemistry, 2004. 76(21): p. 6419-6425.

37. Hempe, J.M., Protein Analysis by Capillary Electrophoresis, in Handbook of Capillary and Microchip Electrophoresis and Associated Microtechniques, J.P. Landers, Editor. 2007. p. 75-107. 



\title{
Chapter II \\ Methods and Techniques
}

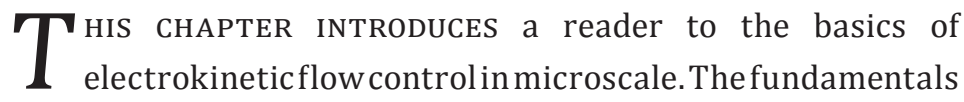 \\ of capillary electrophoresis are briefly presented, followed by \\ a short overview of methods used throughout the experiments \\ presented in this thesis.
}




\section{Capillary electrophoresis}

Capillary electrophoresis (CE) is a term coined to separation of charged species in narrow capillaries containing buffer solution under an applied electric field. To perform CE a capillary or a microfluidic channel is filled with an appropriate separation buffer and a sample is loaded at one of the outlets. Subsequently, high voltage - typically up to $30 \mathrm{kV}$ for traditional systems and several $\mathrm{kV}$ for microchip devices - is applied to the system and species start to migrate. The velocity and the direction of migration is determined by the charge to mass ratio of a component and is given by

$$
v=\frac{q}{6 \pi \eta r} E_{s}=\mu_{e p} E_{s}
$$

where $q$ is the charge of the particle, $\eta$ the viscosity of the buffer and $r$ the radius of the particle. The symbol $\mu_{e p}$ on the right hand side of equation 1 denotes the electrophoretic mobility, which is a commonly used measure used in electrophoresis.

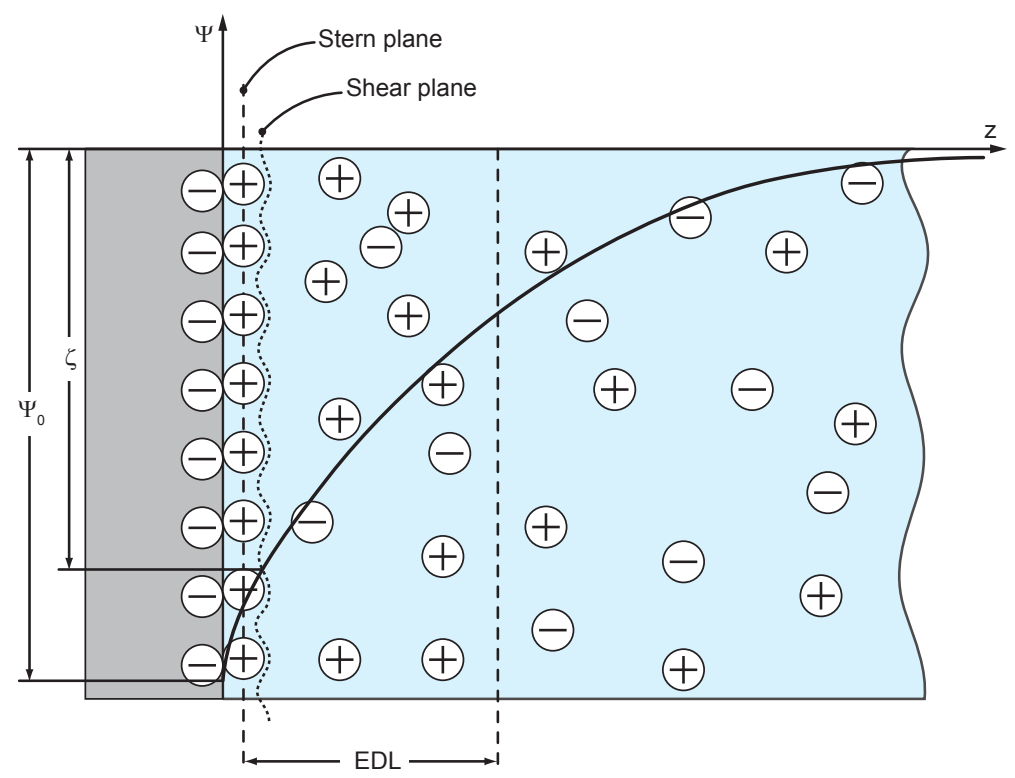

Figure 1. Schematic representation of the interface between a negatively charged capillary wall and an aqueous solution.

When brought in contact with an electrolyte, many materials develop surface charge. In case of glass, the charge is a result of deprotonation of silanol groups. To compensate it, electrolyte ions migrate towards the surface, adsorb to it and become 
immobile. This immobilized layer of ions is called Stern layer. Directly next to it, there is a diffusive layer composed of ions attracted to the surface but still mobile; the distribution of these ions is determined by the electric forces and Brownian motion. Both layers together are referred to as electrical double layer (EDL). When an electric field is applied parallel to the channel surface the ions in the diffusive layer migrate in the direction determined by their net charge, dragging the fluid in the channel. This phenomenon is called electroosmotic flow.

The velocity of the EOF is calculated in a way similar to electrophoretic migration:

$$
v=\frac{\varepsilon_{0} \varepsilon_{r} \zeta}{\eta} E_{S}=\mu_{e o} E_{S}
$$

where $\varepsilon_{0}$ is the permittivity of vacuum, $\varepsilon_{r}$ the relative permittivity of the electrolyte, $\zeta$ - zeta potential (see Figure 1). The symbol $\mu_{e p}$ is referred to as electroosmotic mobility.

The importance of EOF in electrophoretic systems becomes obvious if the fact that different species can carry either positive or negative charge is considered. This would render electrophoretic separation impossible in many scenarios because fractions with opposite charges move in opposite directions upon the application of an electric field. Certainly, there is a way to detect them on both sides of a capillary - the collection is, however, technically challenging. When EOF is combined with electrophoretic migration the species migrate according to the net mobility

$$
v=\left(\mu_{e o}+\mu_{e p}\right) E_{S}
$$

Thus it is possible to observe the migration of the negatively charged species towards the cathode - the phenomena commonly used in capillary zone electrophoresis (Figure 2). 

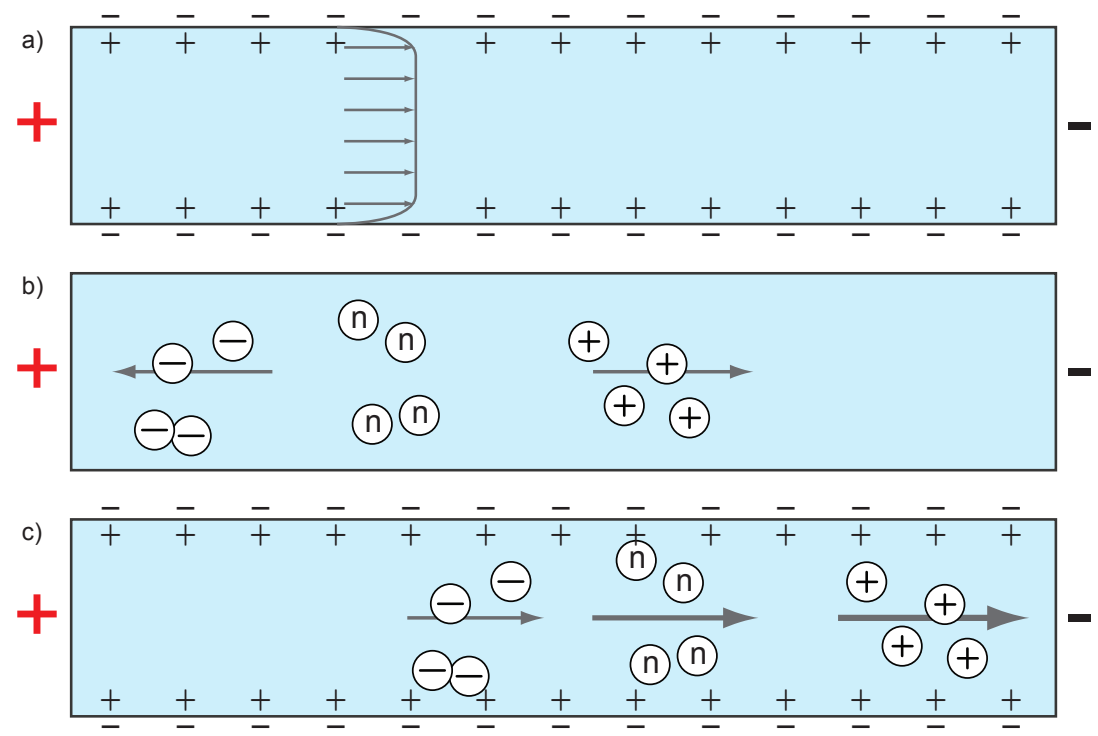

Figure 2. The driving forces of electrokinetic flow: (a) electroosmotic flow; (b) electrophoresis; (c) combination of electroosmotic flow and electrophoresis.

\section{Modelling electrokinetic channel networks}

In contrast to lab-scale instruments, preparative electrophoresis on a chip usually requires more than one channel, unless all operations, including sample injection and retrieval are to be performed manually with a pipette. The absolute minimum is thus an addition of two channels crossed with the main separation channel: one for sample injection and one for pooling the selected fraction. The complexity of the network increases if additional tasks are to be performed. To control the flow direction and velocity in all branches of such fluidic network, an analytical model of the system must be built. There are several detailed studies on modelling electrokinetic networks; ${ }^{1,2}$ the most approach common is to derive an equivalent electric circuit of the device (Figure 3a). ${ }^{3}$ The channels are treated as perfect ohmic conductors and thus usual circuit analysis methods can be applied to solve the model, e.g. if the fluxes in all the channels are known, the electric potentials that need to be applied to induce them can be calculated. 
a)

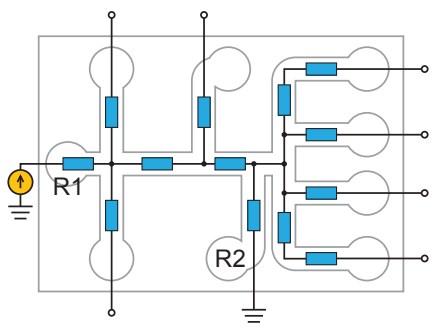

b)

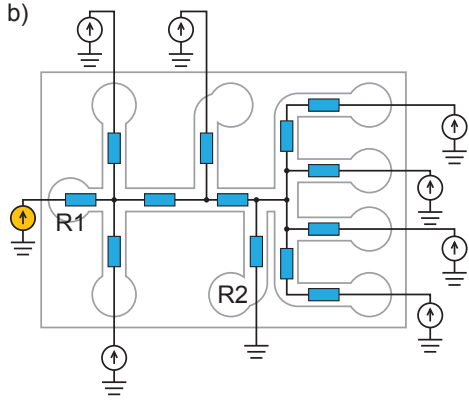

Figure 3. Electrical equivalent circuit representation of a microfluidic network. Flow is supposed to occur from the reservoir R1 to the reservoir R2. (a) Simple case. (b) Biasing of all junctions.

However, such analysis usually doesn't yield the expected results. A common example here is an electrokinetic injection of a sample into a separation channel in a CE chip. In Figure 4a a fluorescence photograph of the injection is shown. The voltages are applied only at the outlets of the loading channel (i.e. outlets of the separation channel are electrically floating). Instead of migrating in the region confined within the junction region, the sample also spreads into the separation channel. This phenomenon is a result of the simplifications used in the electric circuit analogy, which doesn't consider the finite dimensions of the channels at the junctions (circuit nodes) and the diffusional processes. The electrokinetic injection schemes in microchips has been a subject of a number of studies ${ }^{4-6}$ - one of the solutions commonly in use is called a 'gated injection' - that is applying some predefined bias-voltage during an injection to the outlets of the separation channel. A resulting plug shape when using this technique is shown in Figure $4 \mathrm{~b}$.
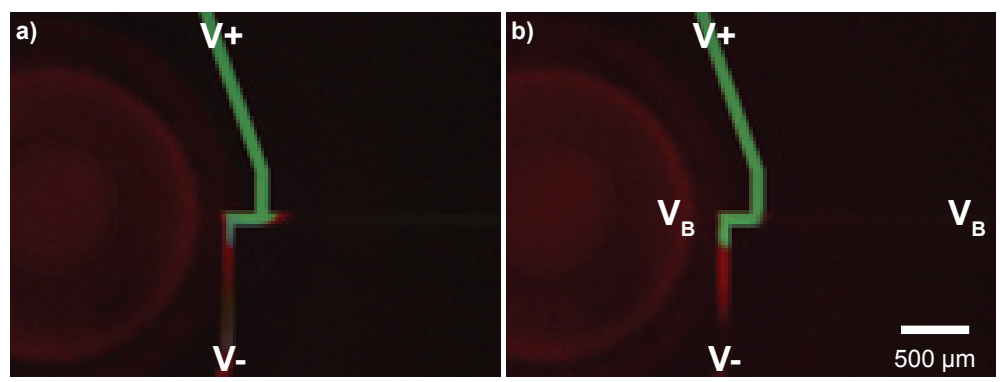

Figure 4. Injection in microchip capillary electrophoresis: (a) unbiased and (b) gated injection.

It is evident that electric circuit analogy must be redefined. In principle, during the operation of a complex electrokinetically controlled channel networks no outlet 
can be left electrically floating -even for a simple operation shown in Figure 3, the biasing scheme presented in Figure $3 \mathrm{~b}$ is more relevant.

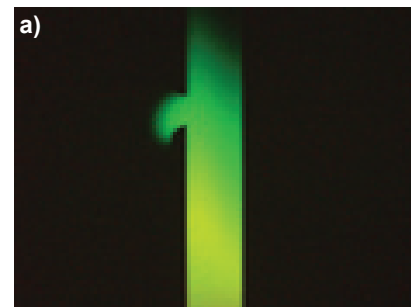

d)

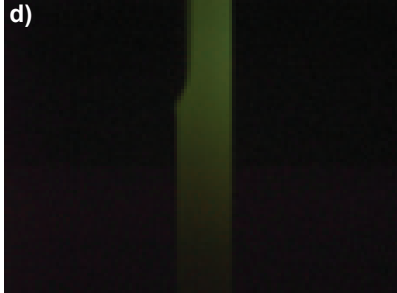

g)

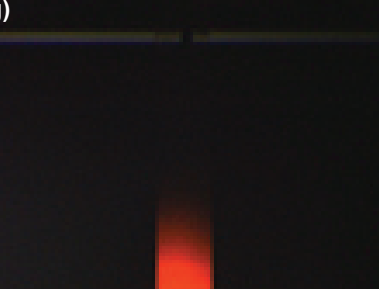

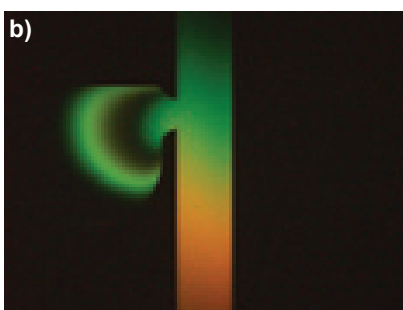

e)

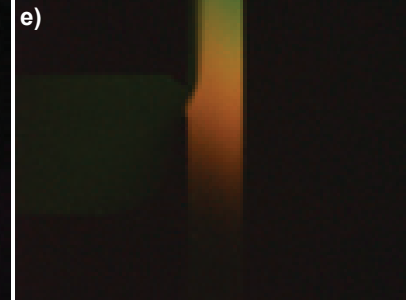

h)

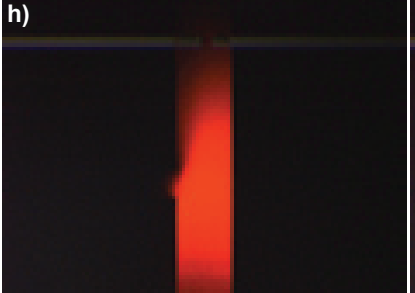

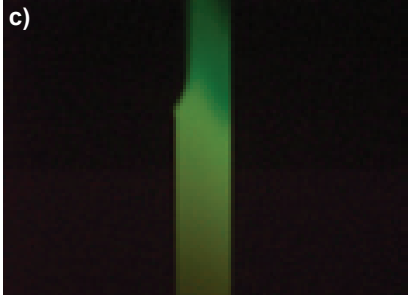

f)

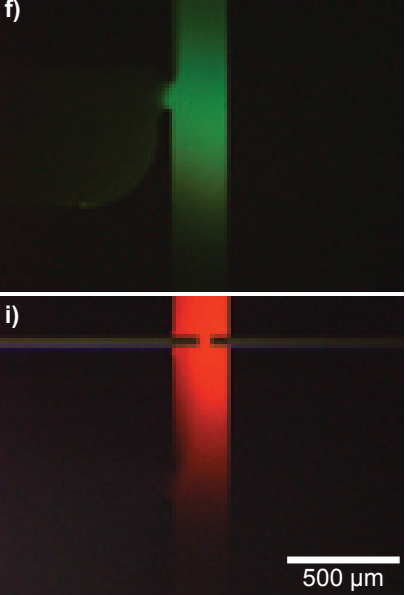

Figure 5. Junction passing by sample fractions. (a,b) No side-channel biasing. (c,d) Overbiasing of the side channel. (e,f) Using same biasing for more mobile (e) and less mobile (f) fraction. (g,h) Application of side channel biasing in real system - the time gap between the frames is $100 \mathrm{~ms}$.

In preparative CE chip devices the situation is even more challenging - the system has to deal with fast moving analyte bands, not a steady flow as during an injection. Figure 5a,b shows fluorescence photographs of a separated fraction passing a T-junction when no biasing of the side-channel takes place. The distortion of the peak and resulting loss of material are clearly visible - if the peak was smaller such undesired behaviour could result in virtually vanishing of the plug. The voltage that must be applied to counteract this process cannot be easily calculated - basically it should be a little higher than a potential measured at the interconnection of the channels when no biasing is applied. However the presence of a new fixed potential in the electric network changes the currents in all branches, thus the circuit must be re-analyzed. Quite commonly overbiasing is observed (Figure 5c,d) which also contributes to the sample plug dispersion. Even if a proper biasing voltage is found, there is still another challenge that remains unsolved - the fractions have different 
mobilities, and a scheme that works perfectly for a more mobile fraction can cause a less mobile fraction to distort. Such situation is illustrated in Figure 5e,f - this effect is not usually a great contributor to the overall plug dispersion unless peak widths are comparable with a width of the junction. Unfortunately, there is no easy analytical way to derive the needed biasing voltages in complex networks and most of solutions need to be determined experimentally.

\section{Peak detection}

In principle, preparative electrophoresis requires continuous real-time information about the locations of all fractions. However, such data cannot be acquired and thus partial information must be sufficient - it is provided by a single detector positioned at some distance from the injection point along the separation channel. There exists a number of detection methods for on-a-chip CE. In the current research fluorescence detection with a photomultiplier tube was employed.

The main challenge is not the choice of the detection method but the real-time peak recognition and derivation of fractions parameters. Obviously, if the migration time and the width of a passing band are known its mobility and diffusivity can be calculated effortlessly. Little research has been devoted to fast peak detection algorithms. Unfortunately, the techniques used commonly for electrophoretic analysis can be hardly applied because they rely on processing of the whole electropherogram. One method described in literature for active systems is the use of the first derivative of a detector signal as a peak presence indicator. ${ }^{7}$ Yet, it yields false results if the signal is noisy.

Instead, we decided to use another algorithm - for the detection of a peak, a weighted running variance computed real-time is compared with the variance of a whole signal acquired to the moment. When a predefined difference between both values is detected the area is marked as a peak-containing region. 


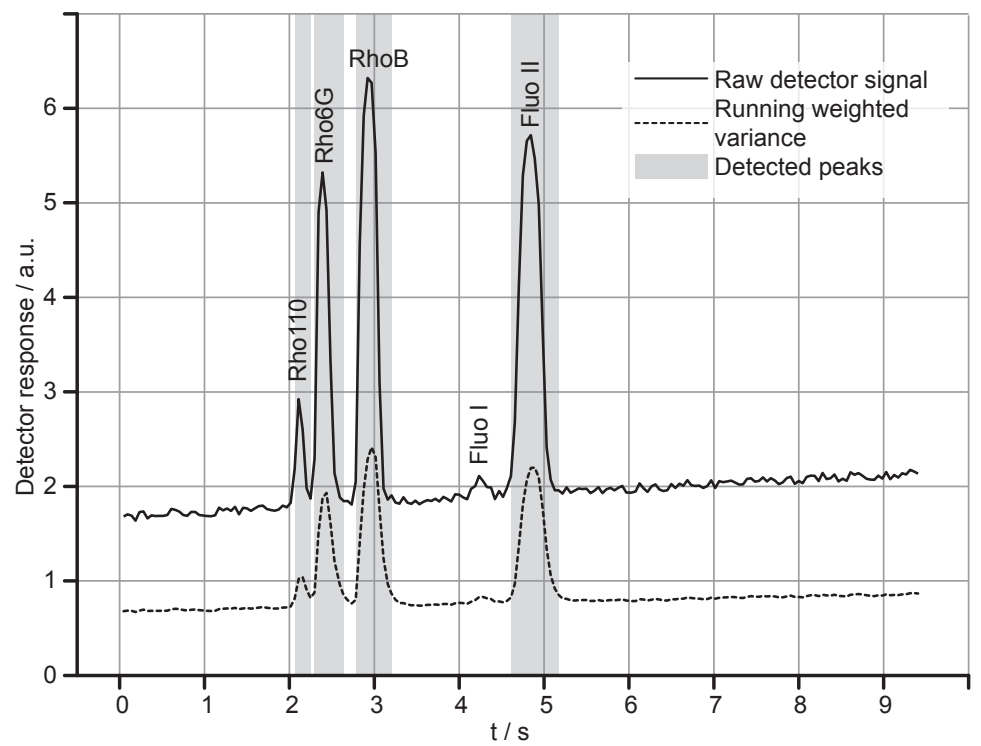

Figure 6. Real-time peak detection in preparative capillary electrophoresis.

\section{High voltage switching}

Additionally to reliable peak detection, there should be a method to deliver fast changing voltages. In this research the high-voltage power supplies manufactured by IBIS BV were used. These instruments are controlled over the RS-232 serial data interface. The overhead of the communication using this protocol, combined with the latency of the power supplies was a serious obstacle in timely switching. Quite commonly in fast burst modulation, the duration of a single period was up to fifty percent longer than pre-programmed.

However, after measuring dynamic responses of the instruments to the requests, it was possible to partly compensate for these effects by appropriate shifting of the switching requests in time. Finally, the accuracy around 40-50 ms could be achieved. 


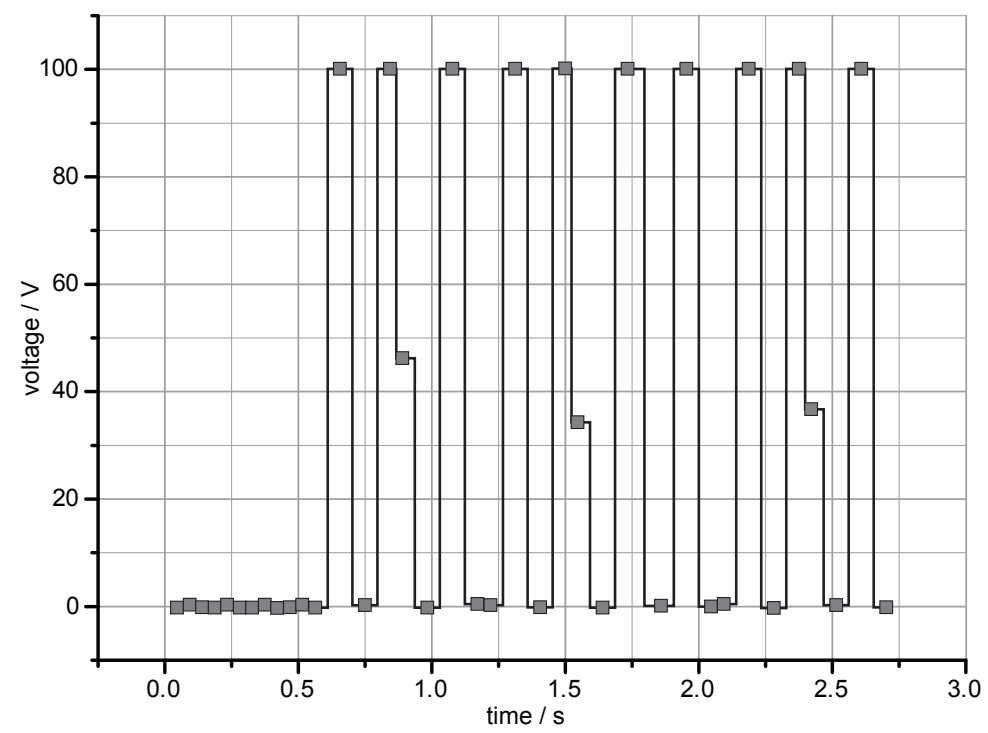

Figure 7. Fast switching in high-voltage power supply.

\section{Software control}

All chip devices described in the following chapters were controlled by $\mu$ Exec - a home-made application written in .NET C\#. The program has a modular composition (see Figure 8) allowing for easy addition of new supported chips and hardware instruments. The main advantage of $\mu \mathrm{Exec}$ is the easiness of operating the chips the only user input that is usually required is defining the connections (i.e. showing which chip outlets are connected to the voltage lines) and writing a steering script in a high-level language. An example of such a script used for operating a micropreparative CE device is shown in Appendix A to this thesis.

After submission, scripts are translated into code understood by the execution module (MXU). The MXU runs on a separate high-priority thread and uses Windows high-resolution performance counters for precise time control - resolution of less than $1 \mathrm{~ms}$ can be easily achieved this way. ${ }^{8}$ If possible, all instrument requests (i.e. commands sent to instruments) are predefined during the script translation, thus there is no overhead in calculations and library calls during the execution. 


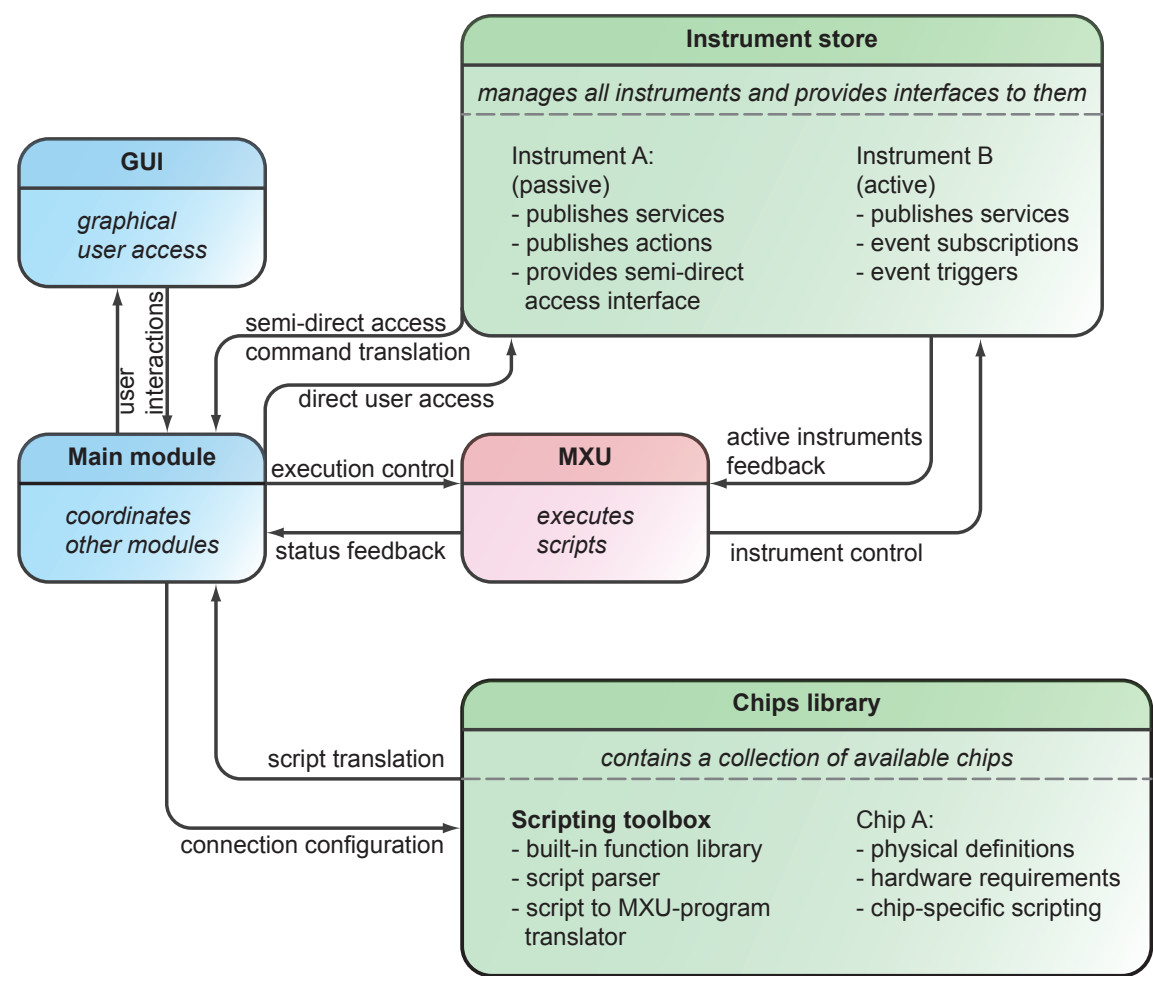

Figure 8. Schematic representation of the main modules of the control application $\mu$ Exec.

\section{References}

1. Xuan, X.C. and D.Q. Li, Analysis of electrokinetic flow in microfluidic networks. Journal of Micromechanics and Microengineering, 2004. 14(2): p. 290-298.

2. Berli, C.L.A., Theoretical modelling of electrokinetic flow in microchannel networks. Colloids and Surfaces a-Physicochemical and Engineering Aspects, 2007.301(1-3): p. 271-280.

3. Berli, C.L.A., Equivalent circuit modeling of electrokinetically driven analytical microsystems. Microfluidics and Nanofluidics, 2008. 4(5): p. 391-399.

4. Tsai, C.H., R.J. Yang, C.H. Tai, and L.M. Fu, Numerical simulation of electrokinetic injection techniques in capillary electrophoresis microchips. Electrophoresis, 2005. 26(3): p. 674-686.

5. Blas, M., N. Delaunay, and J.L. Rocca, Comparative study of floating and dynamic injection modes in electrokinetic separative microsystems. Electrophoresis, 2007. 28(24): p. 4629-4637. 
6. Bias, M., N. Delaunay, and J.L. Rocca, Electrokinetic-based injection modes for separative microsystems. Electrophoresis, 2008. 29(1): p. 20-32.

7. Tulock, J.J., M.A. Shannon, P.W. Bohn, and J.V. Sweedler, Microfluidic separation and gateable fraction collection for mass-limited samples. Analytical Chemistry, 2004. 76(21): p. 6419-6425.

8. About timers. MSDN documentation. Available from: http://msdn.microsoft.com. 



\section{Chapter III \\ Electrokinetic Manipulation of CE Separated Fractions in a 2D Laminar Flow Chamber}

$\mathrm{T}$

HIS CHAPTER PRESENTS an alternative to recently published planar microfluidic devices for post-separation sample manipulation. A method is described by which post-separation sample handling in a two-dimensional planar microfluidic chamber can be performed with a reduced number of steering channels. Contrary to other designs, flow direction is not changed during sample transfer, and the sample is sandwiched between two sheath streams which are adjusted to control position and width of the sample stream. As a result sample fractions are guided one by one into different parallel lanes. The width of fractions during transfer is determined by the separation channel width and focusing rather than by injection volume and diffusion, by which cross-over between collection lanes can be avoided. The presented concept may be applied to deliver a separated sample to a secondary separation column, but also to enable in-situ measurements of separated fractions with optical techniques, where both considerable amount of sample and long measurements time are required. The behaviour of the system under high sample load as well as the feasibility of performing in-chamber sample recirculation by integrated electrodes will be discussed. It is demonstrated that high sample loads are feasible, but are limited by the design geometry. 


\section{Introduction}

Complex samples can rarely be satisfactorily separated by a single method. ${ }^{1,2}$ The problem becomes even more pronounced in microscale separation devices, where resolution is limited due to short separation column lengths. ${ }^{3,4}$ To overcome this difficulty separation techniques are often combined orthogonally to form multidimensional separation systems. Several groups have presented these techniques in miniaturized formats, successful demonstrations include CGE-MEKC, ${ }^{5}$ MEKC-EC, ${ }^{6}$ IEF-CGE, ${ }^{7,8}$ IEF-CZE, ${ }^{9,}{ }^{10}$ EC-CZE. ${ }^{11}$ These multidimensional separation concepts utilize either serial coupling, where only a part of the sample separated in the first dimension is injected into the second dimension (one-to-one transfer), or follow the traditional two-dimensional approach and employ parallelization to achieve one-to-many sample transfer. In either case the first separation dimension serves as a preparative tool for the secondary.

Usually the transport between separation dimensions is achieved by simple mechanical coupling and very little, if any, manipulation of separated fractions occurs. On the other hand microfluidics offers a handful of techniques for precise liquid handling. Most of them rely on either mechanical actuation ${ }^{12,13}$ or droplet transport ${ }^{14-16}$ and very few examples of integrated systems based on these concepts have been shown. ${ }^{17-19}$ Yet, there is the alternative of using electrokinetic flow, and some methods of performing preparative CE in purely electrokinetic devices have been demonstrated. ${ }^{20-24}$ Mostly, channel networks are applied, where separated sample is pooled into individual reservoirs, after passing at least one junction. ${ }^{21,22,25}$ Several problems specific to this format have been addressed and the presented solutions included a.o. optimization of the junction geometry, ${ }^{25}$ electric biasing protocols, ${ }^{26}$ alternative junction passing techniques ${ }^{27}$ and integration of microelectrodes into a junction to reduce dispersion. ${ }^{28}$ The continuous effort to build electrokinetic-only devices is driven by the overall simplicity of such an approach. ${ }^{29,30}$ Since there are no mechanical, moving parts in a miniaturized system, the manufacturing of the device is less complicated, moreover a lack of external actuation, besides electric power, greatly simplifies interfacing and reduces the costs of equipment needed to operate the setup.

Recently, a planar-format microfluidic device was suggested, ${ }^{31,32}$ as an alternative way of post-separation sample manipulation. In this proposal, a traditional junction network is replaced with a planar 2D chamber. After separation, a sample is transferred into the chamber with an aid of electrokinetic focusing and subsequently, 
by reconfiguring the steering voltages, is pushed in the transverse direction towards a parallel channel structure. Such an approach gives more flexibility, as the lane into which the sample goes, can be actively selected (however, with increasing number of components in the sample the choice becomes restricted), and to some extent helps avoiding sample dispersion - a common problem occurring at channel junctions in electrokinetically driven systems.

Here, we present an alternative method of post-separation sample handling in a two-dimensional planar chamber, with a reduced number of steering channels. As opposed to the previous design, the direction of the flow is not changed during the transfer. After entering the chamber the sample is sandwiched in-between two sheath streams. By adjusting the fluxes of the sheath streams, the vertical position and the width of the sample stream can be varied. As a result sample fractions are guided into different parallel lanes, one by one. Since the fractions are handled serially, it is possible to discard some of them, i.e. guide the unwanted portion into a waste lane. Another advantage of this method is that the width of the fractions during the transfer is determined by the separation channel width and focusing, rather than by the injection volume and diffusion process. This way the contamination between the second dimension lanes can be avoided.

We discuss and validate the steering principle of the device and demonstrate its operation as a preparative tool for post-separation sample manipulation. The initial development of the current system was aimed at delivering not only a method for transporting the separated sample to a secondary separation principle, but also to provide a platform which would enable in-situ measurements of separated fractions with optical techniques, where both considerable amount of sample and long measurements time were required. Therefore we also show the behaviour of the system run under high sample load conditions and demonstrate the feasibility of performing in-chamber sample recirculation by integrated electrodes. ${ }^{33}$

\section{Procedures and apparatus}

\section{Chemicals}

Chemicals were obtained from Sigma-Aldrich-Fluka. A $40 \mathrm{mM}$ solution of $\mathrm{N}$-[2-Hydroxyethyl] piperazine-N -[2-. ethanesulfonic acid] (Hepes) at pH 6.35 was used as a buffer. Tween 20 was added $(0.05 \% \mathrm{w} / \mathrm{v})$ to reduce surface tension and help filling the device. The sample consisted of $6 \mathrm{mM}$ fluorescein and $4 \mathrm{mM}$ rhodamine $\mathrm{B}$ in buffer solution. For visualization of sample stream positioning a solution of $10 \mathrm{mM}$ 
fluorescein in buffer was used. Directly before starting experiments solutions were filtered through a $0.22-\mu \mathrm{m}$ membrane filter and degassed for $15 \mathrm{~min}$ in a vacuum chamber.

\section{Chip fabrication}

The chip consisted of two bonded $1.1 \mathrm{~mm}$ thick borosilicate glass plates (Schott Borofloat 33). The top plate contained the fluidic network which was etched in $10 \%$ hydrofluoric acid through a patterned $\mathrm{Cr} / \mathrm{Au}$ mask and reservoir openings which were micro-powder blasted with $29 \mu \mathrm{m} \mathrm{Al}_{2} \mathrm{O}_{3}$ through a thick polymer photoresist foil. The bottom plate contained sputtered $\mathrm{Pt} / \mathrm{Cr}$ electrodes.

\section{Setup and chip operation}

The chip device was placed in a custom-made holder, which provided both electrical and fluidic connections. Two high voltage power supplies (CU-411, IBIS BV, The Netherlands), with four independently operated channels each, delivered the potentials needed for electrokinetic flow generation. The power supplies were connected to a personal computer and controlled by an in-house written native Windows application with time resolution of $40 \mathrm{~ms}$. An inverted microscope (Olympus IX-51) equipped with UV light source and fluorescence filter set (XF-57) was used for visualization. The images were captured with a digital camera (ColorView II) mounted to the microscope and controlled by Analysis software. The numerical simulations were carried out in ESI-CFD software; the chip device was simplified to a 2-D model. The electroosmotic mobility for the purpose of simulations was assumed to be $5 \cdot 10^{-8} \mathrm{~m}^{2} \cdot(\mathrm{Vs})^{1}$.

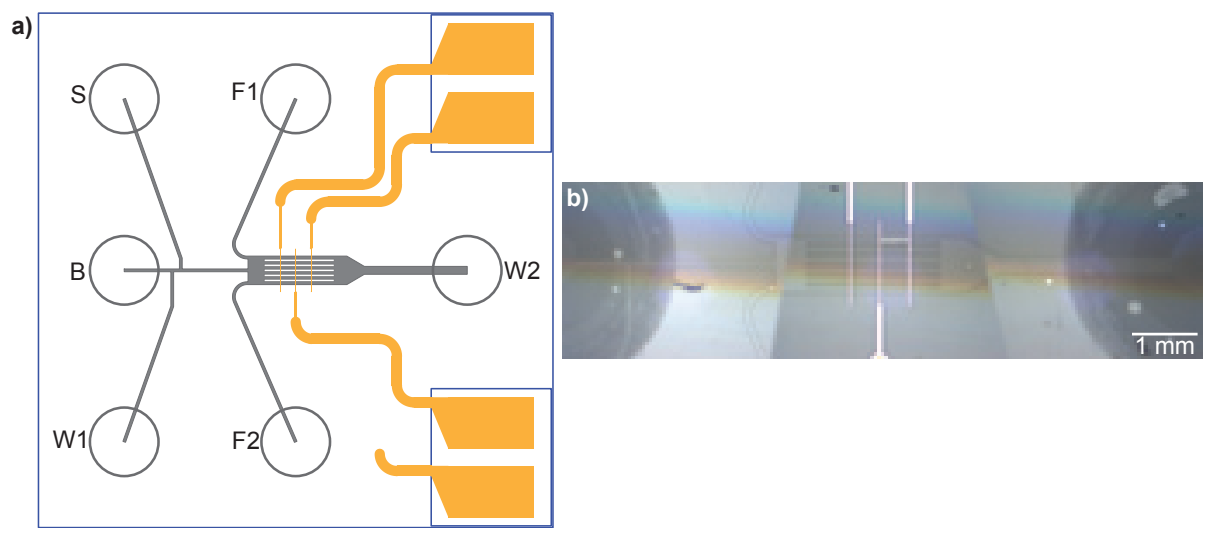

Figure 1. (a) Schematic of the microfluidic device consisting of a CE part and a sample manipulation region. (b) Transmitted light image of the central part of the fabricated device. 
A schematic of the microfluidic chip is shown in Figure 1. The channels were 100 $\mu \mathrm{m}$ wide and $10 \mu \mathrm{m}$ deep, except the channel connected to the W2 reservoir, which was $250 \mu \mathrm{m}$ wide. The planar chamber was $850 \mu \mathrm{m}$ wide and consisted of four sections: the $500 \mu \mathrm{m}$ long entrance section, the parallel lanes structure of $2000 \mu \mathrm{m}$ in length, the tapered region of $500 \mu \mathrm{m}$ in length, and the $400 \mu \mathrm{m}$ long exit section.

The device can be considered as a CE separation channel coupled to a laminar flow chamber with controllable vertical sample stream position. CE separation was performed by first injecting the sample into the separation channel (using $\mathrm{S}$ as a sample reservoir and $\mathrm{W} 1$ as a waste sink) and then establishing the electric field by applying a potential difference between the buffer source (B) and the waste reservoir (W2). Gated injections were used unless otherwise indicated with loading time of $0.75 \mathrm{~s}$ and the separation field strength was $333 \mathrm{~V} \cdot \mathrm{cm}^{-1}$. During the separation, sample progressed along the channel towards the chamber and after entering it was guided into one of the horizontal lanes that are present in the chamber. The switching of the sample stream position (i.e. varying the lane into which the sample was directed) was possible by sandwiching it between sheath streams with varying fluxes. ${ }^{34}$ This process was controlled, based on the computational model, by applying appropriate potentials to the reservoirs F1 and F2. To achieve spatial separation of fractions resolved in a single CE run, by guiding them into different lanes, the sample stream position was changed rapidly as the fractions appeared at the entrance of the chamber.

\section{Model of operation}

The electric potentials applied to the reservoirs, needed to produce a given vertical sample stream position were derived with a simplified model of the device. Figure 2 shows a schematic of the laminar flow chamber with six parallel lanes and an equivalent electric circuit ${ }^{35}$ used for calculations.

The following assumptions were made in the model: (i) the density, electrokinetic mobility and electric conductivity of all fluids present in the device are constant; (ii) the electric field at the chamber entrance is uniform and contains the longitudinal component only, thus this part is modelled by a single resistor $R_{C 1}$ (iii) the crosssections of the channels are rectangular; (iv) the flow switching is instantaneous. 
a)

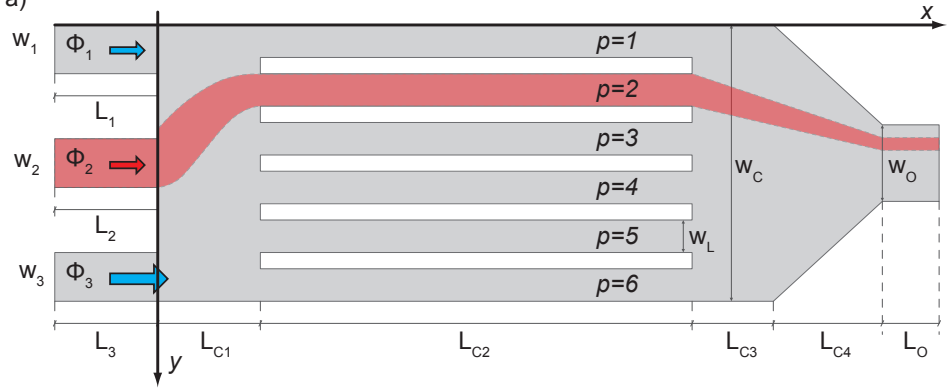

b)

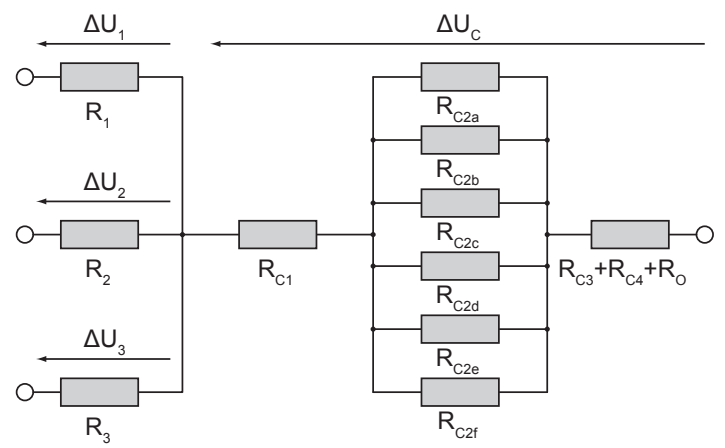

Figure 2. (a) Schematic of a 2D sample manipulation region with corresponding dimensions. (b) Electric equivalent circuit used for flow control.

The following operating parameters must be provided for the calculations: the average flow velocity in the lanes $u_{L}$, the sample stream position $\mathrm{p}$ (i.e. the lane through which the sample stream flows - see Figure 2a) and the sample stream confinement coefficient $\alpha$-defined as a fraction of the width of an in-chamber lane, for $\alpha=1$ the sample stream occupies the full width of the lane. With these parameters known, the average flux through a lane can be defined

$$
\phi_{L}=u_{L} \cdot w_{L} \cdot d
$$

where $w_{L}$ is the width of the lane and $d$ is the depth of the channels. On the assumption that all fluid densities in the chip are equal, application of the law of conservation of mass then yields the fluxes for the chamber inlet channels

$$
\begin{aligned}
& \phi_{1}=\left(p-1+\frac{1-\alpha}{2}\right) \phi_{L} \\
& \phi_{2}=\alpha \phi_{L} \\
& \phi_{3}=\left(N-p+\frac{1-\alpha}{2}\right) \phi_{L}
\end{aligned}
$$


where $\mathrm{N}$ is the number of the parallel lanes in the chamber. The voltage drop along the channel needed to produce the appropriate electrokinetic flow velocity for a given flux can be calculated with

$$
\Delta U=\frac{\Phi L}{\mu_{0} A}
$$

where $\Phi$ is the flux through a channel, $L$ - the length of a channel, $A$ - cross-sectional area of the channel and $\mu_{0}$ - the electroosmotic mobility of the buffer. After calculating the voltage drops along the inlet channels $\Delta U_{1}, \Delta U_{2}$ and $\Delta U_{3}$, the total electric current flowing through the chamber is given as a sum of all the inlet currents:

$$
i_{0}=\sum_{i=1}^{3} \frac{\Delta U_{i}}{R_{i}}
$$

With all the currents known, it is straightforward to derive the potentials that are needed to be applied to the inlet channels by using the Kirchhoff's laws and the Ohm's law. ${ }^{35}$

\section{Results and discussion}

\section{Sample stream positioning}

The validation of the steering model was carried out with a sample containing fluorescein in buffer solution. The sample was placed in reservoir B1 and continuous flow of the sample through the separation channel was forced by applying a potential difference between B1 and W2. The position of the sample stream in the chamber was controlled by applying voltages derived from the model to the reservoirs B, F1, F2 and W2. Figure 3 shows a comparison of the measured sample stream positions versus the predicted values for different steering voltages. The experimental data was obtained by fitting concentration profiles measured at a distance $x=2900 \mu \mathrm{m}$ from the beginning of the chamber. The difference between the observed and the predicted positions increases as the sample stream was located further from the middle of the chamber and reached a maximum of around $\Delta y=0.018$ - a relatively small value. However, even such a small displacement error during the manipulation of separated fractions can be significant, as it can lead to serious contamination of adjacent lanes. 


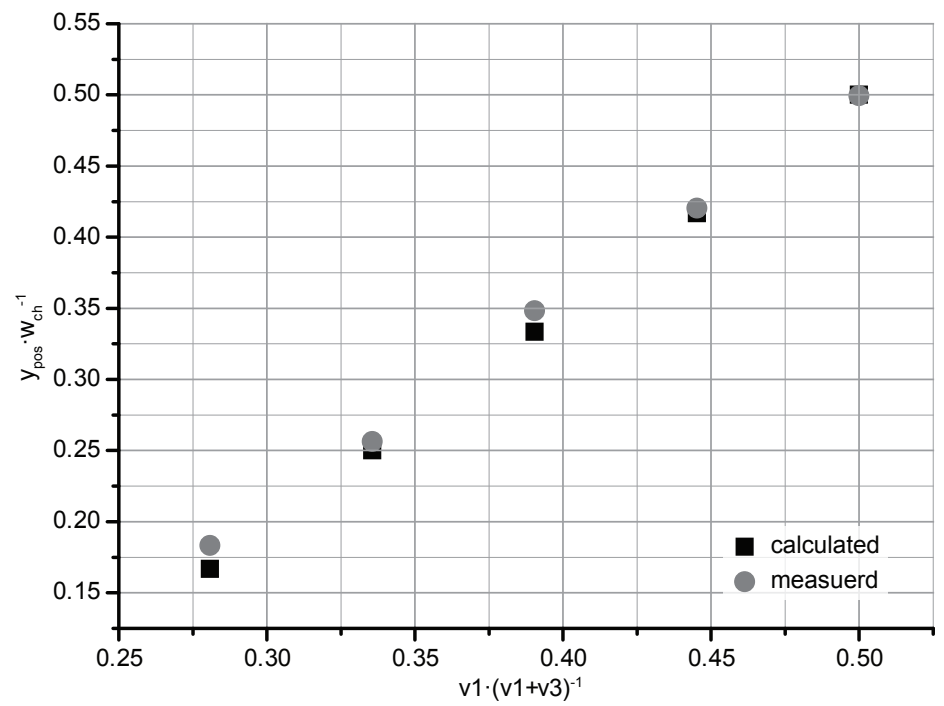

Figure 3. Calculated and measured sample stream position for varying sheath streams voltages. The difference between the predicted and measured values increases with the stream deflection. V1 and V 3 are potentials applied the sheath streams reservoirs $\mathrm{F} 1$ and $\mathrm{F} 2$, respectively.

The differences between the measurements and the theoretical values are mostly caused by the simplifications used in the model of the device. In the calculations it was assumed that the electric field in the entire chamber is uniform and has a longitudinal component only. However, simulations of potential distribution and streamlines (Figure 4a) reveal that the electric field varies greatly in the vicinity of the chamber entrance and the sample stream does not reach its position directly at the beginning of the chamber but rather slowly deflects towards the correct position. Moreover molecular diffusion, which is not restricted by walls in the entrance region of the chamber, contributes to the overall sample dispersion and worsens the situation.

To overcome the problem of positioning, we conducted a series of experiments, trying to find the optimal steering parameters. The following values of the position parameter $p$ used in equation (2) produced a proper, contamination-free guiding of the sample stream: lane $2-p=1.92$; lane $3-p=2.96$; lane $4-p=4.04$; lane $5-p=5.09$. Moreover the sample stream was focused to 0.74 of its original width to counteract the diffusion related spreading. Figure 5 shows fluorescence images of sample stream positioning for parameters as calculated from the model and for the corrected parameter values. 
a)

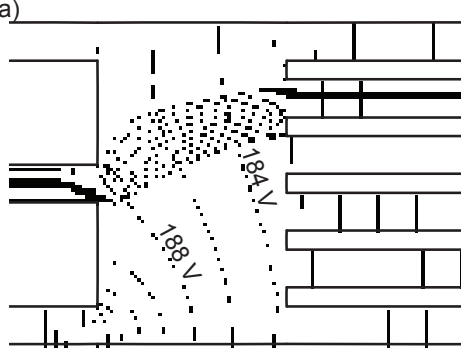

b)

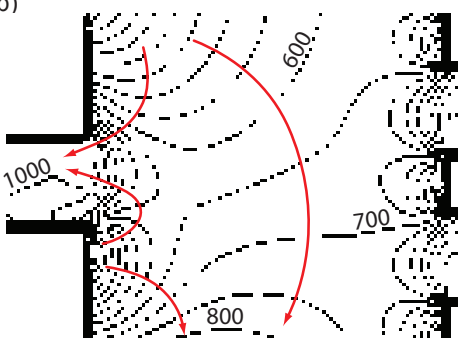

Figure 4. (a) Simulation of the equipotential lines and streamlines for position $p=2$. Great variations in the electric field strength in the chamber entrance region are observed - a contradiction to the model, where uniformity of the electric field is assumed. (b) Simulation of isovelocity lines in the vicinity of the sample inlet in the chamber (numeric values in $\mu \mathrm{m} \cdot \mathrm{s}^{-1}$ ) - arrows point in the direction of the increasing velocity magnitude. The sample stream moves much faster than the fluid in the chamber.

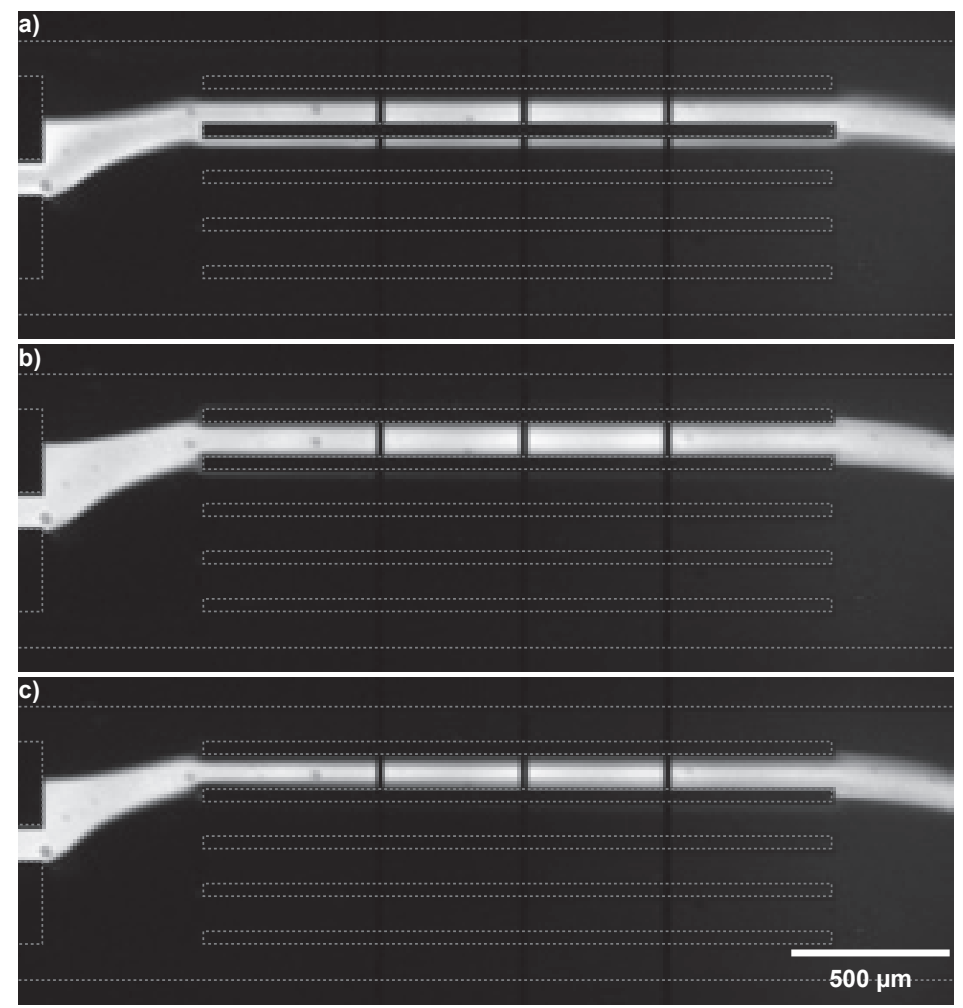

Figure 5. Fluorescence images of sample stream positioning. (a) Voltages applied as derived directly from the model for position $p=2$. (b) Voltages calculated for a corrected position value $p=1.9$. (c) Voltages applied for a corrected position value $p=1.9$ and sample stream confinement $\alpha=0.74$. 


\section{Manipulation of separated fractions}

The manipulation of the separated fractions was demonstrated on a rhodamine B / fluorescein mixture. First a sample plug of $325 \mu \mathrm{m}$ in length was injected into the separation channel. After applying an electric field of $333 \mathrm{~V} \cdot \mathrm{cm}^{-1}$, separation started. At this moment pull-back voltages were applied to the S1 and $\mathrm{W} 1$ reservoirs. The separation times of both fractions were measured at the moment of arrival of a fraction at the chamber entrance. Based on this information, the timing in the steering script was set - the separation with simultaneous guiding of the sample stream into the second lane lasted for $2.25 \mathrm{~s}$ and was followed by directing the sample stream into the third lane for $2.5 \mathrm{~s}$. The in-lane velocity during these steps was $13 \mathrm{~mm} \cdot \mathrm{s}^{-1}$ and $11 \mathrm{~mm} \cdot \mathrm{s}^{-1}$ respectively. Similar instructions were repeated for a following separation, with guiding the fractions into the fourth and fifth lanes. Figure 6 shows a sequence of fluorescence images taken during this experiment. Guiding of the separated fractions into different lanes for two consecutive separations is shown. Upon entering the chamber, the fraction shapes distorted (see e.g. Figure 6a,c,g,h) but eventually they focused and entered the preprogrammed lanes without contaminating the adjacent channels. The deformation of the plugs during the injection into the chamber is caused by variations in electric field strength in this region. Especially, the difference in the velocity magnitudes (Figure $4 \mathrm{~b}$ ) between the fast moving sample stream and relatively slow moving fluid in the chamber causes the plug to take an arrowhead-like shape observed e.g. in Figure 6c. The contribution of this effect to the overall lengthening of fraction plugs can be estimated, on the assumption that plug concentration profiles adhere to the Gaussian function, from

$$
\sigma_{\text {total }}^{2}=\sigma_{\text {init }}^{2}+\sigma_{d}^{2}+\sigma_{f}^{2}+\sigma_{c h}^{2}
$$

where $\sigma_{\text {total }}^{2}$ is the total variance of a plug; $\sigma_{\text {init }}^{2}$ is the initial variance measured at the end of the separation channel; $\sigma_{d}^{2}$ is the variance due to diffusion; $\sigma_{f}^{2}$ is the variance due to focusing and $\sigma_{c h}{ }^{2}$ is the component that includes all the effects not included in the preceding terms, that contribute to the distortion of the plug during entering the chamber. We found that in the case of the experiments presented here the initial variance of a plug, measured at the end of the separation channel, just before entering the chamber, increased on average for the plugs positioned in the middle of the lanes by a factor of $\sigma_{\text {total }}{ }^{2} \cdot \sigma_{\text {init }}{ }^{-2}=4.15$ for the lanes 3 and 4 , and $\sigma_{\text {total }}{ }^{2} \cdot \sigma_{\text {init }}{ }^{-2}=4.52$ for the lanes 2 and 5 . In both cases the variance due to manipulation in the entrance region equaled 0.57 of the total variance increase. 


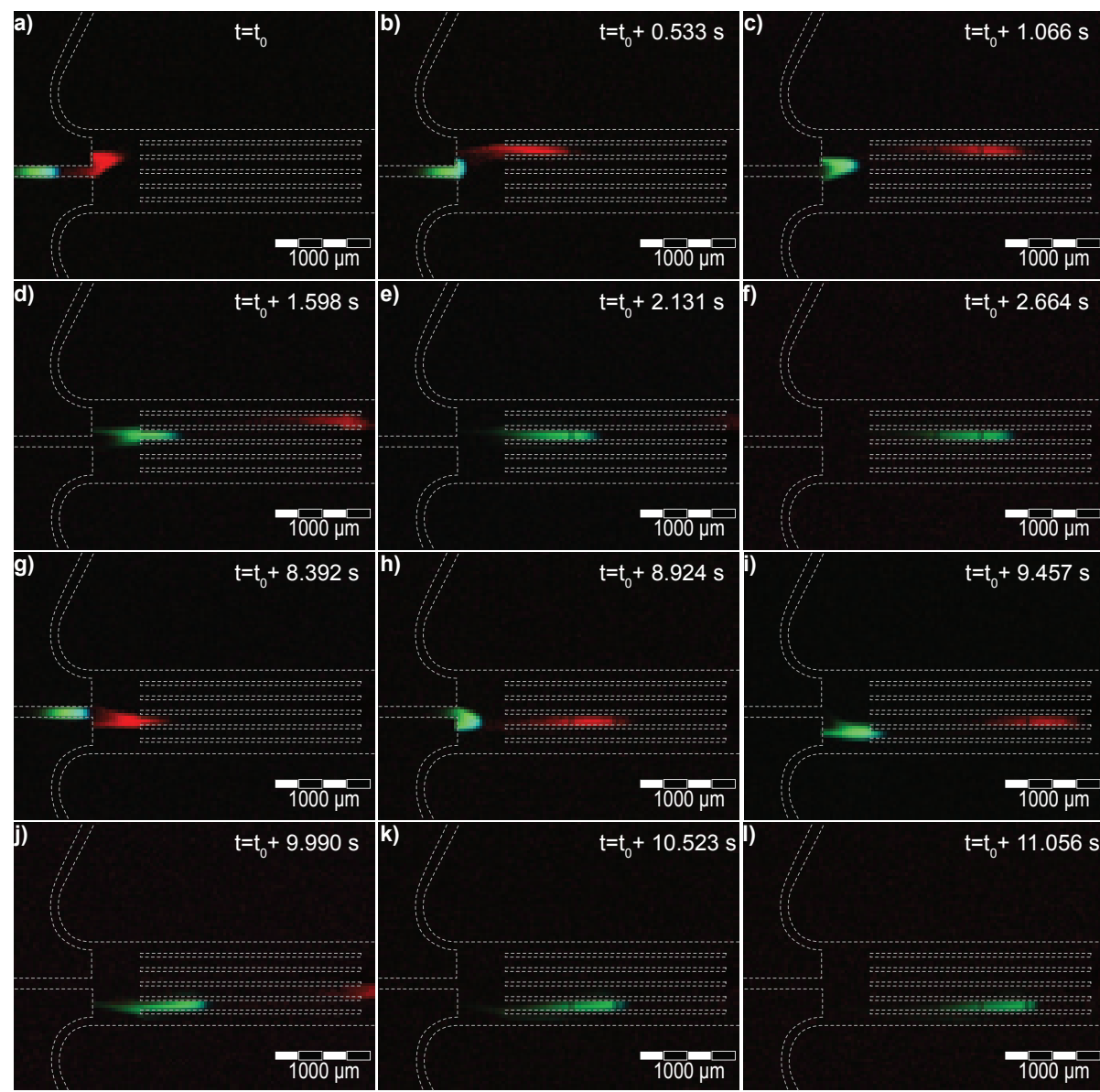

Figure 6. Sequence of fluorescence images of 2D manipulation of CE separate. Two consecutive separations are shown (the delay between the separation was $\mathbf{2 . 5}$ s). (a-c) Component I of separation I directed into $2^{\text {nd }}$ lane. (d-f) Component II of separation I directed into $3^{\text {rd }}$ lane. ( $\left.\mathrm{g}-\mathrm{i}\right)$ Component I of separation II directed into $4^{\text {th }}$ lane. $(j-l)$ Component II of separation II directed into $5^{\text {th }}$ lane.

This is a relatively large ratio, and taking into account the high diffusivity of the model mixture used in the experiments, it can grow even larger when another sample is used. Therefore, the lengthening of the separated plugs due to the manipulation must always be taken into account when this technique is used.

\section{Performance with high sample load}

One important factor that determines the quality of an electrophoretic separation is the sample volume that is injected into a separation channel. Usually increasing the sample load reduces the separation resolution. However, in preparative techniques the quantity of the fraction material obtained in a single run is often of 
greater importance than the ability to resolve all the sample components. To test the sample loading limits of the device, we disabled biasing during the injection procedure (i.e., the side channels during the sample injection were electrically floating). Additionally the injected plug was allowed to spread before the separation started for a fixed amount of time. This way the length of the injected plug could be controlled without altering the device geometry. By systematically increasing this length, it was found that when it reached a value of around $400 \mu \mathrm{m}$, correct guiding of the fractions became difficult. Figure 7 shows a sequence of images taken during the manipulation of fractions separated with an injected plug length of $450 \mu \mathrm{m}$. The main challenge in this situation is to switch the sample stream position at a proper instant, presumably after the first fraction has completely entered its lane.

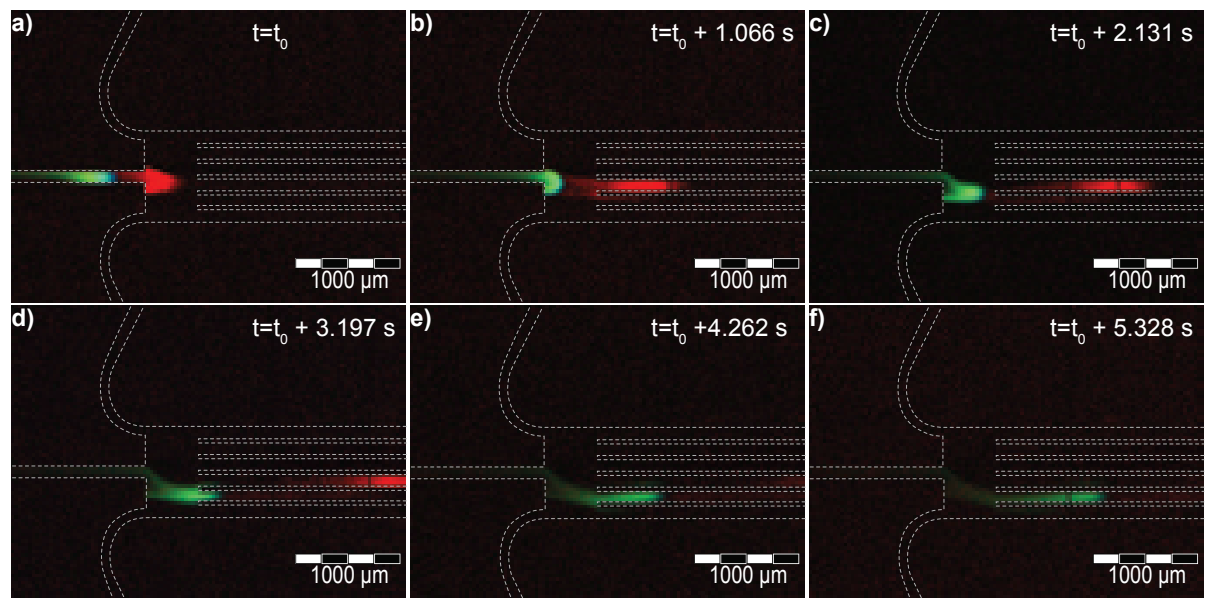

Figure 7. Sequence of fluorescence images of 2D manipulation of a CE separated sample with sample overloading. The much larger fractions cannot be steered properly, causing contamination of adjacent channels during manipulation.

However, in the case depicted, this switching is impossible: the fractions nearly overlap and their length much exceeds the length of the entrance region of the chamber. The only procedure that is applicable in such a scenario is to start switching the lanes at the latest moment possible, when the preceding fraction did not fully enter its lane but the second component advanced in the chamber already and must be guided into another lane. Nevertheless, the result of manipulation was unsatisfactory, because switching started before the transfer of the first plug was finished, parts of it entered the lane designated for the upcoming fraction, and also some material of the second component was pushed into the channel belonging to the first one. The results clearly show that the assumption about instantaneous flow switching is not valid - particularly the desired change of the sample position per 
time unit cannot be larger than the maximum lateral velocity of the sample under given conditions

$$
\frac{d y}{d t} \leq \mu E_{y}
$$

Therefore it becomes evident that the geometrical arrangement becomes one of the most important design considerations in system following the manipulation method described here. Particularly, the length of the chamber entrance region should be adjusted to the length of injected sample plugs and to the diffusional dispersion occurring during the separation.

\section{Manipulation with sample recirculation}

The three integrated electrodes that crossed the chamber: one in the middle of the chamber and two others positioned $450 \mu \mathrm{m}$ to the right and to the left of it (see Figure 1) were used to engage sample recirculation. Such a technique enables mixing of a sample in a well-defined channel segment, which is beneficial if only small sample volumes are available and prolonged residence time is required for the detection ${ }^{33}$ (e.g. following binding kinetics by optical methods). During the transfer of the separated fractions, after the first component reached the middle of its lane, the flow in the device was stopped and the recirculation started by applying a potential of $2 \mathrm{~V}$ to the outer electrodes and grounding the middle one. ${ }^{33}$ After a predefined recirculation time elapsed, the remaining fraction was pushed into another lane and the recirculation procedure was repeated. Unfortunately, a performance test showed that our design is unsuitable for such an approach. After the first fraction was positioned in the recirculation region, the second component already entered the chamber, and because of lack of mechanical barriers, diffused significantly. Our efforts to squeeze it back to its original width by employing forced focusing with sheath streams proved unsuccessful and therefore contamination was unavoidable. Figure 8 shows a sequence of fluorescence images taken during one of these experiments - the recirculation of rhodamine B fraction (Figure 8b,c) lasted only 2 seconds; yet the fluorescein plug diffused considerably. The 'squeezing' sequence applied to reshape this plug included rapid focusing with confinement coefficient of $\alpha=0.5$ and guiding into the $4^{\text {th }}$ lane for 0.6 seconds (i.e. pushing the fraction one lane lower than its target lane), followed by guiding into lane 3 with $\alpha=0.7$. However, this procedure did not fully counteract the diffusion and serious contamination of the second lane occurred. 

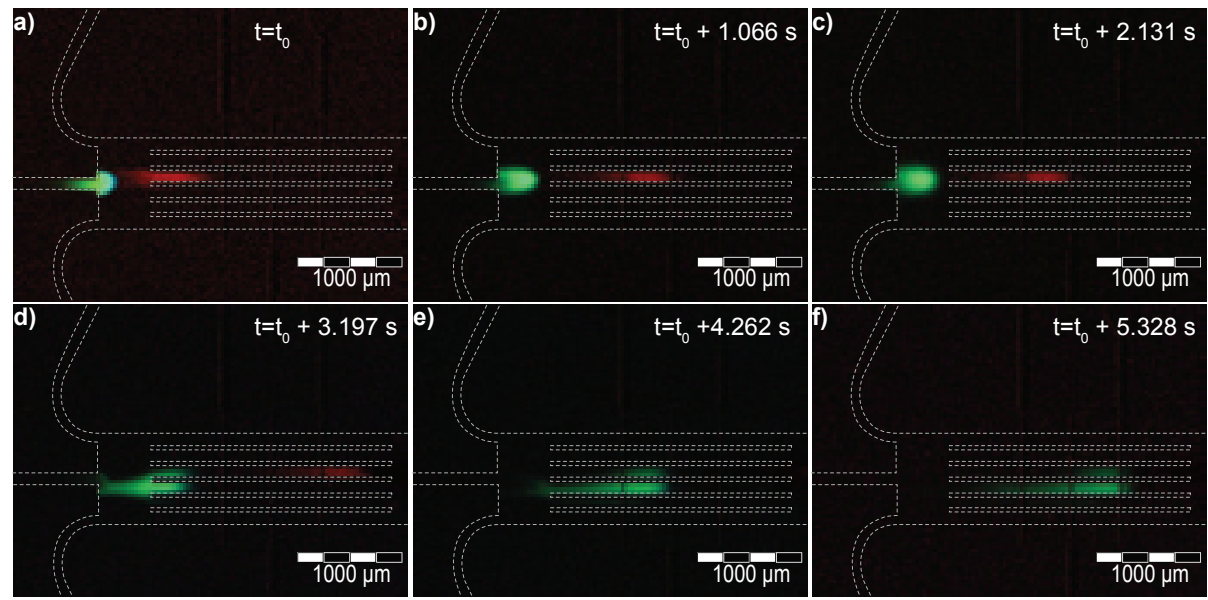

Figure 8. Sequence of fluorescence images of 2D manipulation of a CE separated sample with stopping of the components. After the first component reaches the position between the electrodes the flow is stopped (all electric connections floating) and the sample is recirculated for $2 \mathrm{~s}$. The flow is then resumed and the second component is directed into the $3^{\text {rd }}$ channel.

\section{Conclusions}

In conclusion, we demonstrated a method of planar electrokinetic sample transport and manipulation after electrophoretic separation. In the proposed approach only two additional steering channels are required to achieve a flexible serial handling mechanism. Additionally the method has an advantage of being able to deal with much longer fractions than previously known designs, and the ability to do so is mainly limited by the design geometry.

\section{Symbols}

$\Phi_{L}$ - flux through a lane

$u_{L}$ - velocity of flow in a lane

$w_{L}$-width of a lane

$d$ - depth of the fluidic network

$\Phi_{1,3}$ - inlet sheath streams fluxes

$\Phi_{2}$ - inlet sample stream flux

$p$ - lane number to which the sample stream is steered

$\alpha$-sample stream confinement in a lane

$\mu_{0}$-electroosmotic mobility of a buffer 
$A$ - cross-sectional area of a channel

$i_{0}$-electric current flowing through the chamber

\section{References}

1. Adkins, J.N., S.M. Varnum, K.J. Auberry, R.J. Moore, N.H. Angell, R.D. Smith, D.L. Springer, and J.G. Pounds, Toward a human blood serum proteome - Analysis by multidimensional separation coupled with mass spectrometry. Molecular \& Cellular Proteomics, 2002. 1(12): p. 947-955.

2. Nice, E.C., J. Rothacker, J. Weinstock, L. Lim, and B. Catimel, Use of multidimensional separation protocols for the purification of trace components in complex biological samples for proteomics analysis. Journal of Chromatography A, 2007. 1168(1-2): p. 190-210.

3. Culbertson, C.T., S.C. Jacobson, and J.M. Ramsey, Microchip devices for high-efficiency separations. Analytical Chemistry, 2000. 72(23): p. 5814-5819.

4. Das, C. and Z.H. Fan, Effects of separation length and voltage on isolectric focusing in a plastic microfluidic device. Electrophoresis, 2006. 27(18): p. 3619-3626.

5. Shadpour, H. and S.A. Soper, Two-dimensional electrophoretic separation of proteins using poly(methyl methacrylate) microchips. Analytical Chemistry, 2006. 78(11): p. 3519-3527.

6. Rocklin, R.D., R.S. Ramsey, and J.M. Ramsey, A microfabricated fluidic device for performing two-dimensional liquid-phase separations. Analytical Chemistry, 2000. 72(21): p. 5244-5249.

7. Griebel, A., S. Rund, F. Schonfeld, W. Dorner, R. Konrad, and S. Hardt, Integrated polymer chip for two-dimensional capillary gel electrophoresis. Lab on a Chip, 2004. 4(1): p. 18-23.

8. Li, Y., J.S. Buch, F. Rosenberger, D.L. DeVoe, and C.S. Lee, Integration of isoelectric focusing with parallel sodium dodecyl sulfate gel electrophoresisformultidimensional protein separations in a plastic microfludic network. Analytical Chemistry, 2004. 76(3): p. 742-748.

9. Herr, A.E., J.I. Molho, K.A. Drouvalakis, J.C. Mikkelsen, P.J. Utz, J.G. Santiago, and T.W. Kenny, On-chip coupling of isoelectric focusing and free solution electrophoresis for multidimensional separations. Analytical Chemistry, 2003. 75(5): p. 1180-1187.

10. Cong, Y.Z., L.H. Zhang, D.Y. Taol, Y. Liang, W.B. Zhang, and Y.K. Zhang, Miniaturized two-dimensional capillary electrophoresis on a microchip for analysis of the tryptic digest of proteins. Journal of Separation Science, 2008. 31(3): p. 588-594. 
11. Gottschlich, N., S.C. Jacobson, C.T. Culbertson, and J.M. Ramsey, Two-dimensional electrochromatography/capillary electrophoresis on a microchip. Analytical Chemistry, 2001. 73(11): p. 2669-2674.

12. Grover, W.H., R.H.C. Ivester, E.C. Jensen, and R.A. Mathies, Development and multiplexed control of latching pneumatic valves using microfluidic logical structures. Lab on a Chip, 2006. 6(5): p. 623-631.

13. Oh, K.W. and C.H. Ahn, A review of microvalves. Journal of Micromechanics and Microengineering, 2006. 16(5): p. R13-R39.

14. Pollack, M.G., R.B. Fair, and A.D. Shenderov, Electrowetting-based actuation of liquid droplets for microfluidic applications. Applied Physics Letters, 2000. 77(11): p. $1725-1726$.

15. Chiou, P.Y., H. Moon, H. Toshiyoshi, C.J. Kim, and M.C. Wu, Light actuation of liquid by optoelectrowetting. Sensors and Actuators a-Physical, 2003. 104(3): p. 222-228.

16. Teh, S.Y., R. Lin, L.H. Hung, and A.P. Lee, Droplet microfluidics. Lab on a Chip, 2008. 8(2): p. 198-220.

17. Li, M.W. and R.S. Martin, Integration of continuous-flow sampling with microchip electrophoresis using poly(dimethylsiloxane)-based valves in a sealed device reversibly sealed device. Electrophoresis, 2007. 28(14): p. 2478-2488.

18. Wang, Y.C., M.N. Choi, and J.Y. Han, Two-dimensional protein separation with advanced sample and buffer isolation using microfluidic valves. Analytical Chemistry, 2004. 76(15): p. 4426-4431.

19. Dodge, A., E. Brunet, S.L. Chen, J. Goulpeau, V. Labas, J. Vinh, and P. Tabeling, PDMSbased microfluidics for proteomic analysis. Analyst, 2006. 131(10): p. 1122-1128.

20. Kaniansky, D., M. Masar, M. Dankova, R. Bodor, R. Rakocyova, M. Pilna, M. Johnck, B. Stanislawski, and S. Kajan, Column switching in zone electrophoresis on a chip. Journal of Chromatography A, 2004. 1051(1-2): p. 33-42.

21. Sun, K., Z.Y. Li, K. Ueno, S. Juodkazis, S. Noji, and H. Misawa, Electrophoretic chip for high-fidelity fractionation of double-stranded DNA. Electrophoresis, 2007. 28(10): p. $1572-1578$.

22. Zalewski, D.R., S. Schlautmann, R.B.M. Schasfoort, and H.J.G.E. Gardeniers, Electrokinetic sorting and collection of fractions for preparative capillary electrophoresis on a chip. Lab on a Chip, 2008. 8(5): p. 801-809.

23. Lin, R., D.T. Burke, and M.A. Burns, Selective extraction of size-fractioned DNA samples in microfabricated electrophoresis devices. Journal of Chromatography A, 2003. 1010(2): p. 255-268. 
24. Khandurina, J., T. Chovan, and A. Guttman, Micropreparative fraction collection in microfluidic devices. Analytical Chemistry, 2002. 74(7): p. 1737-1740.

25. Li, G., R. Ran, J.L. Zhao, and Y.S. Xu, Design, simulation, and optimization of a miniaturized device for size-fractioned DNA extraction. Electrophoresis, 2007. 28(24): p. 4661-4667.

26. Yang, S., J. Liu, and D.L. DeVoe, Optimization of sample transfer in two-dimensional microfluidic separation systems Lab on a Chip, 2008. 8: p. 1145-1152.

27. Tulock, J.J., M.A. Shannon, P.W. Bohn, and J.V. Sweedler, Microfluidic separation and gateable fraction collection for mass-limited samples. Analytical Chemistry, 2004. 76(21): p. 6419-6425.

28. Lin, R., D.T. Burke, and M.A. Burns, Addressable Electric Fields for Size-Fractioned Sample Extraction in Microfluidic Devices. Analytical Chemistry, 2005. 77(14): p. 4338-4347.

29. Wu, D.P., J.H. Qin, and B.C. Lin, Electrophoretic separations on microfluidic chips. Journal of Chromatography A, 2008. 1184(1-2): p. 542-559.

30. Peng, Y.Y., A. Pallandre, N.T. Tran, and M. Taverna, Recent innovations in protein separation on microchips by electrophoretic methods. Electrophoresis, 2008. 29(1): p. 157-178.

31. Lerch, M.A. and S.C. Jacobson, Electrokinetic fluid control in two-dimensional planar microfluidic devices. Analytical Chemistry, 2007. 79(19): p. 7485-7491.

32. Lerch, M.A., M.D. Hoffman, and S.C. Jacobson, Influence of channel position on sample confinement in two-dimensional planar microfluidic devices. Lab on a Chip, 2008. 8(2): p. 316-322.

33. Lammertink, R.G.H., S. Schlautmann, G.A.J. Besselink, and R.B.M. Schasfoort, Recirculation of nanoliter volumes within microfluidic channels. Analytical Chemistry, 2004. 76(11): p. 3018-3022.

34. Besselink, G.A.J., P. Vulto, R.G.H. Lammertink, S. Schlautmann, A. van den Berg, W. Olthuis, G.H.M. Engbers, and R.B.M. Schasfoort, Electroosmotic guiding of sample flows in a laminar flow chamber. Electrophoresis, 2004. 25(21-22): p. 3705-3711.

35. Berli, C.L.A., Equivalent circuit modeling of electrokinetically driven analytical microsystems. Microfluidics and Nanofluidics, 2008. 4(5): p. 391-399. 



\section{Chapter IV \\ Forced Splitting of Fractions in \\ Capillary Electrophoresis}

Iractions of analytes on a microfluidic chip, without the need for a longer separation channel, we propose and demonstrate a preparative electrokinetic procedure by which overlapping or closely spaced fractions are automatically split. The method involves an extra T-junction at the end of a separation channel and detector-triggered reconfiguration of voltages at channel outlets. Forced splitting of a separated four-component mixture is demonstrated, and possible sources of errors leading to contamination of split fractions is also discussed in detail and illustrated both by computational fluid dynamics and experiments. The splitting method can be applied in preparative CE-on-a-chip systems, for which it greatly simplifies downstream fraction manipulation and helps in reducing crosscontamination between collected fractions. 


\section{Introduction}

Capillary electrophoresis, CE, is one of the most widespread techniques in analytical sciences. It is utilized for separation of biological samples including DNA, RNA, proteins and peptides. ${ }^{1-5}$ The introduction of microfluidic CE devices in the early nineties ${ }^{6,7}$ revolutionized the field - since then the miniaturization has been a growing area, especially in life science applications. The advantages of the small-scale systems, i.e., low sample consumption and fast analysis times, greatly contributed to the popularity of this approach. ${ }^{8}$ Yet, despite clear benefits many challenges had to be addressed to make miniaturized CE successful. New materials, ${ }^{9}$ sample handling procedures ${ }^{10,11}$ and detection techniques ${ }^{12-16}$ were proposed to target problems specific to the microscale format.

As the miniaturized systems development matures the need to integrate more functions on a chip emerges. Consequently, CE often becomes part of a larger microfluidic system, finding its place as an analytical ${ }^{17-19}$ or preparative p $^{20}$ tool. CE employed in preparative mode can be used to provide separated sample for e.g., multidimensional separations, (bio)chemical reactions, identification, or sequencing. ${ }^{21}$ The ability to select and manipulate a single (pure) fraction is a prerequisite for performing any post-separation processing of a sample. A few systems demonstrating such handling have been described, ${ }^{22-26}$ most of them showing extraction of selected fractions into individual containers.

However, such devices usually have to deal with problems not encountered in common microfluidic CE applications. For example, the high speed of separation, or rather, the high velocity of fractions moving through the separation column, makes precise handling difficult due to the required fast switching of liquid streams. Furthermore, a high sample load, which is preferred for preparative purposes, will decrease separation resolution; this, however, may be solved by performing many consecutive injections and separations in one channel, ${ }^{27}$ or performing separations in parallel channels, requiring reproducibility in timing or manufacturing, respectively. Finally, complex channel networks require special electrokinetic valving techniques and a high level of automation and real-time active control of the system are unavoidable.

In preparative CE it may happen that separation is not satisfactory and sample fractions overlap or are too closely spaced for timely handling in a consecutive step (e.g. re-direction in a side channel, or a 2 nd dimension separation). In such scenarios it is advantageous to perform a pre-fractionation step to increase the 
distance between neighboring sample fractions. In this paper we present a method for automated splitting of adjacent fractions separated by CE. The proposed technique requires an additional T-junction at the end of a separation channel. Splitting is achieved by detector-triggered reconfiguration of voltages as the fraction passes the junction, ${ }^{28,29}$ which resembles the heart-cut technique used in liquid chromatography. ${ }^{30,31}$ We give optimization tips, show the forced splitting of a separated four-component mixture and discuss problems still to solve. The advantage of this method of forced splitting is that it increases spatial separation between fractions without the need for a longer separation channel, therewith reducing the footprint of the chip. In preparative CE-on-a-chip systems the method greatly simplifies downstream fraction manipulation and helps reducing crosscontamination between collected fractions.

\section{Methods and apparatus}

\section{Principle of forced splitting}

For performing the automated spatial splitting of CE separated sample fractions a junction is added at the end of a separation channel. Also a detector is placed at the centre of the junction (at $x=0$ in Figure 1 ).

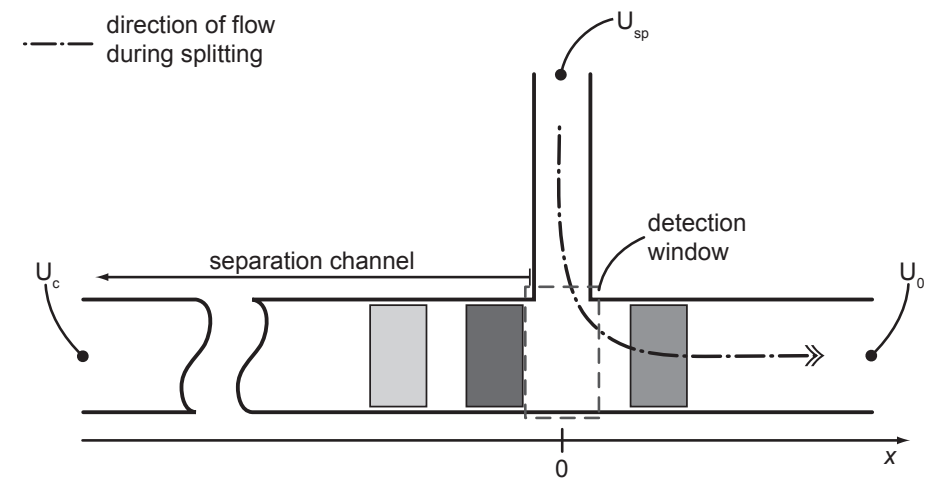

Figure 1. A schematic representation of the forced splitting technique. Fractions residing in the separation channel to the left of the junction $(x<0)$ are idle, while the fraction at the right side of the junction $(x>0)$ is being split by applying an electric field between $U_{s p}$ and $U_{0}$.

During the separation the voltages $U_{c}$ and $U_{0}$ are applied to establish the separation field $E_{s}$, where for the purpose of this study it is assumed that $U_{c}>U_{0}$. When a fraction reaches the end of the separation channel $(x=0)$ a change in the signal is registered by the detector and the fraction is recognized by an appropriate 
algorithm. After the fraction passes the junction completely, the separation is stopped and voltages are reconfigured to produce an electric field between $U_{s p}$ and $U_{0}$ and induce flow from the side channel towards the outlet (see Figure 1). At this moment no voltage is applied to $U_{c}$. The newly established electric field distribution forces the fraction that passed the junction to move further downstream, while the rest of the separated components stay idle in the separation channel. After a desired spacing between the moving fraction and the junction is reached, the voltages are switched back to their original values and the separation is resumed. Subsequently, the whole procedure may be repeated for the remaining fractions.

\section{Materials}

All chemicals were obtained from Sigma-Aldrich-Fluka. Stock solutions of $10 \mathrm{mg} / \mathrm{mL}$ of rhodamine $\mathrm{B}$, fluorescein, $5 \mathrm{mg} / \mathrm{mL}$ of rhodamine $6 \mathrm{G}$ and $2.5 \mathrm{mg} / \mathrm{mL}$ of rhodamine 110, $100 \mathrm{mM}$ 2-(N-morpholino)ethanesulfonic acid (MES) and histidine were prepared in demineralized water. The stock solutions were used to make $20 \mathrm{mM}$ MES/histidine buffer at pH 6.3 and $0.1 \mathrm{mM}$ rhodamine 110, rhodamine $\mathrm{B}$, rhodamine 6G, $0.05 \mathrm{mM}$ fluorescein in $20 \mathrm{mM}$ MES/histidine sample solution, which also contained $3 \%$ ethanol $(\mathrm{v} / \mathrm{v})$ to improve the solubility of the components. ${ }^{32,33}$ Prior to experiments all mixtures were filtered with a $0.22 \mu \mathrm{m}$ membrane filter and degassed in vacuum for 15 minutes.

\section{Device fabrication}

The preparative CE chip was made of two $1.1 \mathrm{~mm}$ thick borosilicate glass plates, which were thermally bonded at $600^{\circ} \mathrm{C}$. The top plate contains the fluidic channels, etched $10 \mu \mathrm{m}$ deep in 10\% hydrofluoric acid through a $\mathrm{Cr} / \mathrm{Au}$ mask layer, and reservoir openings, powderblasted with $29 \mu \mathrm{m} \mathrm{Al}_{2} \mathrm{O}_{3}$ particles. The bottom plate is used as a transparent cover and contains no structures.

\section{Instrumentation and modelling}

The chip was placed in a custom-made holder that provided both electrical and fluidic connections. High-voltage, four-channel power supplies (CU 411, IBIS Technologies, Hengelo, the Netherlands) served as voltage sources. The fluorescence intensity measured by a photomultiplier tube at the point marked with symbol ' $\mathrm{D}$ ' in Figure 2 was used for detection. The detector signal was acquired with a multimeter (Agilent 34401A). All instruments were controlled by a native Windows application, written in-house, and the whole setup operated at $25 \mathrm{~Hz}$ frequency. An inverted microscope (Olympus IX51) equipped with a mercury lamp, fluorescent filter set 
(XF57, Omega Optical, USA and 11012v2, Chroma Technology, USA) and a 32 bit colour CCD camera (ColorView II, Olympus) controlled by Analysis software package (Olympus Soft Imaging Solutions) was used for visualization. Numerical modelling was carried out in the ESI-CFD software package. The following assumptions were made in the simulations: (i) A 2D model was used - since the electric field, velocity and sample concentration are nearly uniform in the $z$ direction, such simplification does not introduce noticeable errors and greatly reduces computation time. (ii) Thermal effects (e.g. Joule heating) were completely neglected, as heat transport in microfluidic devices is usually fast. ${ }^{34}$ (iii) The initial concentration profiles of the sample plugs had box-car shapes instead of more realistic Gaussian shapes. (iv) The conductivity of all fluids was equal.

\section{Results and discussion}

\section{Preparative CE chip}

Figure 2 shows the channel network structure of the device (a) and a microscopic photograph of a section of the chip with the splitting T-junction and marked detector aperture (b). The separation channel is $8.36 \mathrm{~mm}$ long, $100 \mu \mathrm{m}$ wide and $10 \mu \mathrm{m}$ deep. Pinched injections were used with a sample injection volume of $120 \mathrm{pL}$. The $\mathrm{S}$ and W1 reservoirs are used as sample source and waste respectively. The reservoir B1 contains buffer. The channel marked with SP is used for performing forced splitting (with reservoir B2 serving as a buffer source). After the splitting the fractions are driven into the reservoir W2. The fluorescence detector aperture is shown in Figure $2 \mathrm{~b}$ as a dashed circle.
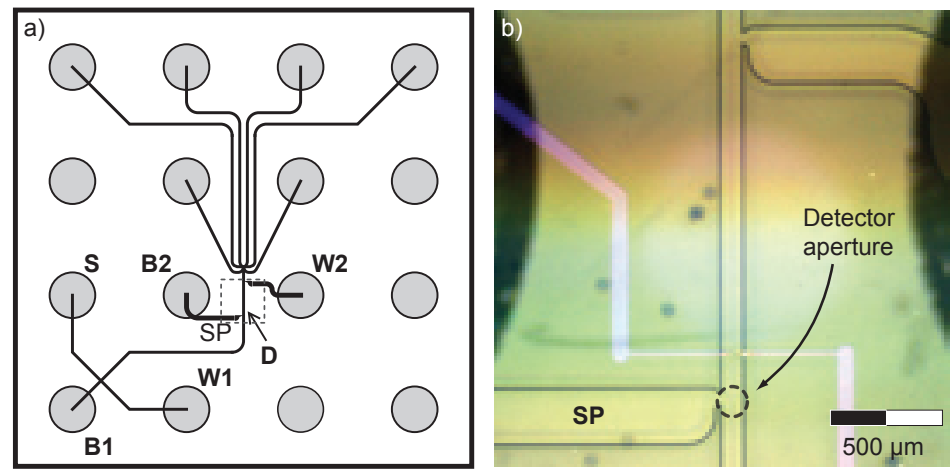

Figure 2. Chip device used in the experiments. (a) Channel network structure. (b) Microscopic photograph of the section of the chip where splitting occurs. The detector aperture is marked with a dashed circle and the splitting channel is marked with 'SP'. 


\section{Optimal splitting conditions}

First we investigated the optimal operation conditions. A proper electric potential balance at the junction is crucial for successful splitting. The electric field penetration to the electrically floating channels at a T-junction ${ }^{10,35,36}$ affects $^{2}$ the sample plug shape of the fraction residing in the vicinity of the junction, and as a result can cause an increase of plug width and loss of analyte. To research this phenomenon in the configuration used for splitting, examine its influence on the performance and choose optimal conditions, a series of numerical simulations was carried out. We modeled the system as a simple T-junction with dimensions shown in Figure 3.

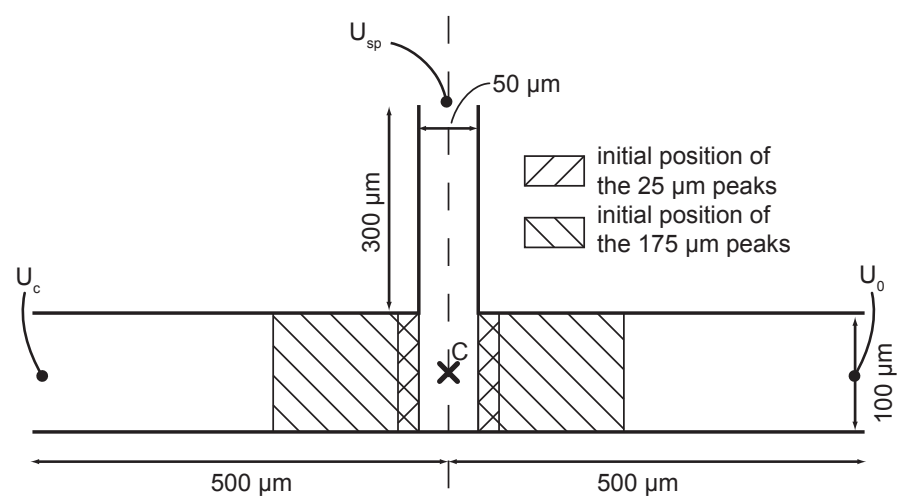

Figure 3. T-junction geometry used in the numerical simulations.

The simulations were performed for peaks with boxcar concentration profiles. Two cases were studied: for the initial peak widths of (i) $25 \mu \mathrm{m}$ and (ii) $175 \mu \mathrm{m}$. The EO (electroosmotic) mobility was assumed to be $1 \cdot 10^{-8} \mathrm{~m}^{2} \cdot(\mathrm{Vs})^{-1}$. The fractions had molar mass $\mathrm{M}=479 \mathrm{~g} \cdot \mathrm{mol}^{-1}$, diffusion coefficient $D=1 \cdot 10^{-10} \mathrm{~m}^{2} \cdot \mathrm{s}^{-1}$, and carried one positive electric charge. The fractions were initially positioned as shown in Figure 3. The transient behaviour of the fractions after application of the splitting potentials was simulated for several voltage configurations. The $U_{s p}$ voltage was set to $250 \mathrm{~V}$, the $\mathrm{U}_{0}$ voltage to $0 \mathrm{~V}$ (these values were chosen to mimic the splitting electric field strength in the separation channel present in the experimental setup), and the $U_{c}$ voltage was varied over the simulations in the range of $U_{c} \cdot U_{j}^{-1}=0 \div 1.5$, where $U_{j}$ is the potential measured at point $\mathrm{C}$ in Figure 3 for the situation when no voltage is applied to the left inlet of the separation channel (i.e. $U_{c}$ floating).

In Figure 4 contour plots of peak positions and concentrations at $\Delta t=100 \mathrm{~ms}$ after starting the splitting procedure are shown. Fraction concentration profiles are 
depicted as colour-gradient values. The distance along the channel $x$ is normalized by the junction width and thus dimensionless. The compensation voltage values $U_{c}$ are divided by the voltage $U_{j}$ defined above. It can be seen, particularly in Figure $4 \mathrm{a}$, that when no compensation voltage is applied ( $U_{c}$ floating) the fraction in the left channel is dragged from its initial position and enters the right channel. Figure 5 shows the electric potential distribution in the vicinity of the junction and the stream lines in such a situation.
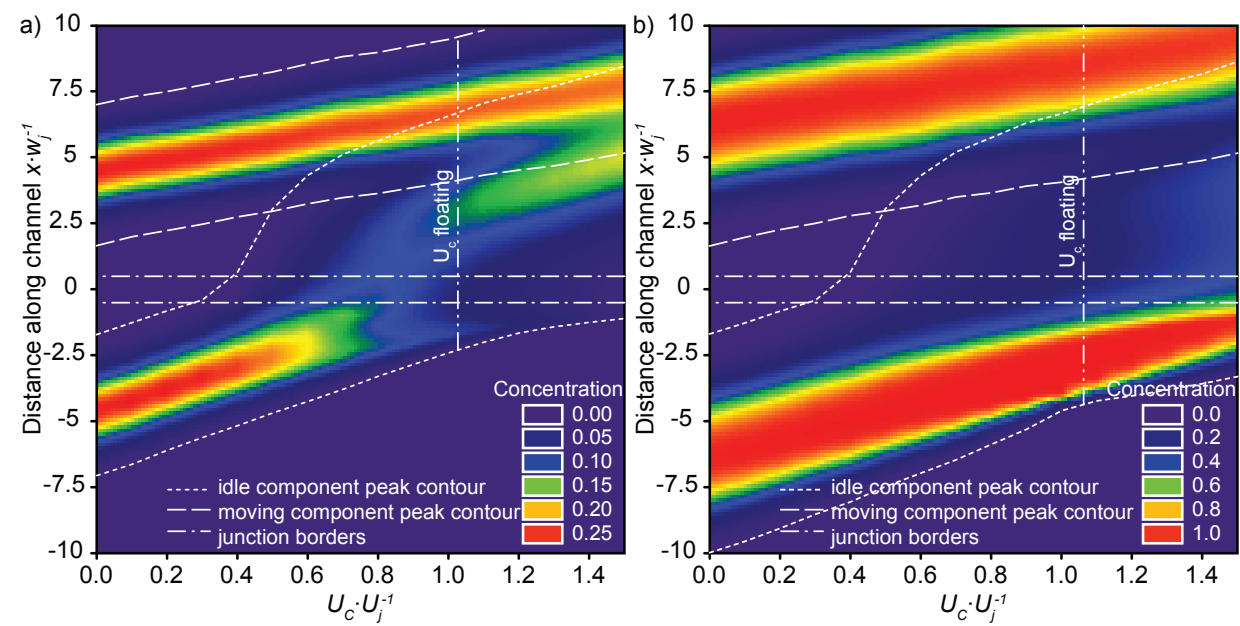

Figure 4. Concentration contour plots of the fractions in the channel after $100 \mathrm{~ms}$ from the moment of the application of the splitting voltage. The concentrations are shown for the compensation voltage range $U_{c} \cdot U_{i}^{-1}=0 \div 1.5$. Situations are shown for (a) $25 \mu \mathrm{m}$ and (b) $\mathbf{1 7 5} \mu \mathrm{m}$ initial plug widths. (Concentration values are presented as fractions of the initial concentration; the differences in intensities between plots (a) and (b) are due to use of a different concentration scale)

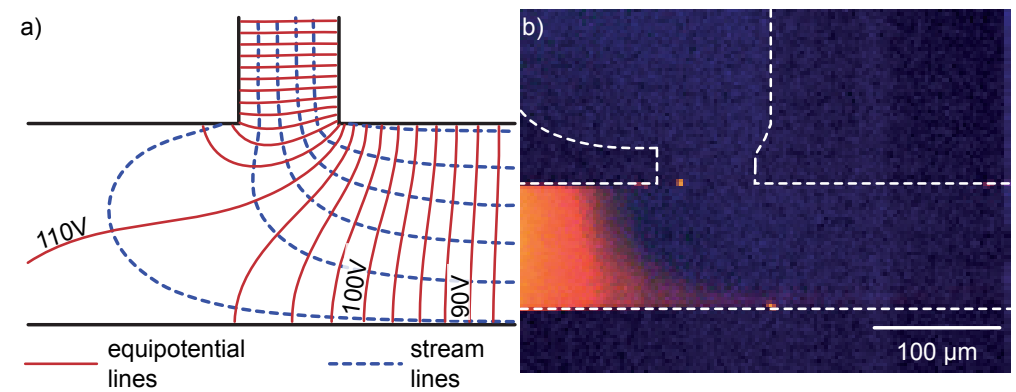

Figure 5. Splitting of the fractions with no pull-back voltage applied. (a) Electric potential distribution and streamlines at the junction. (b) Microscopic picture of Rhodamine B fraction staying idle in the left channel during splitting - the concentration dispersion caused by the electric field spreading out in the left channel can clearly be observed. 
The electric field penetrates into the left channel and thus induces electrokinetic flow in this region. As a result, the fraction supposed to stay idle, is being dragged into the junction area and dispersed. The diffusion significantly contributes to this effect: the fraction of material that diffuses towards the junction is also being swept away, causing even greater dispersion. To avoid this, a pull-back compensation voltage must be applied to the left channel to force the idle fraction to move away from the junction. The required magnitude of the potential depends on several parameters: the mobility and diffusivity of the component, its initial position and the duration of the splitting.

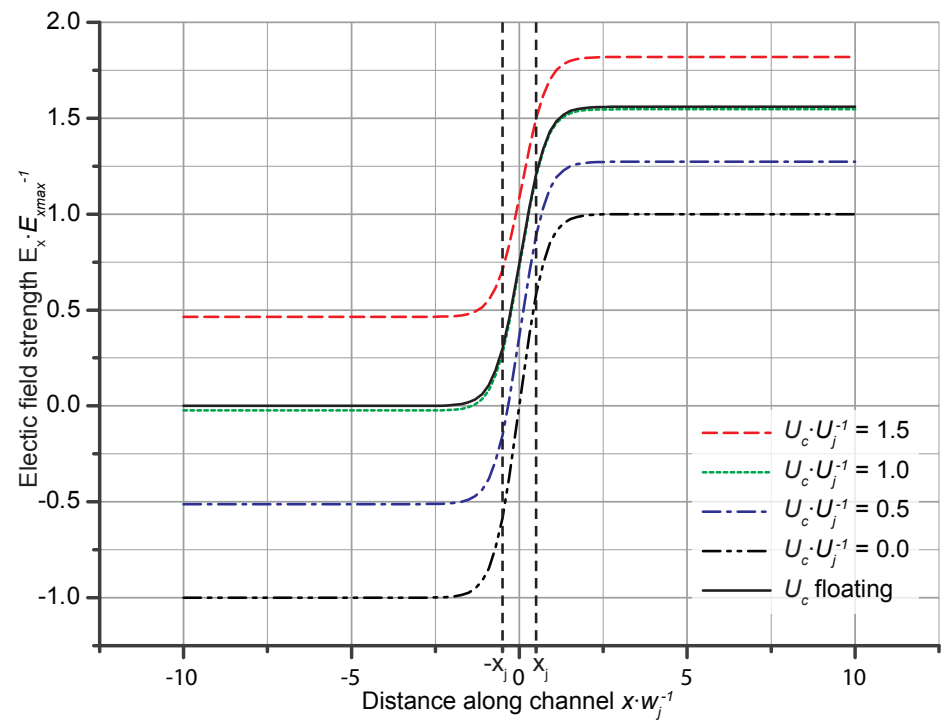

Figure 6. Plots of electric field strength along the separation channel for different potential configurations. The values are scaled by the maximum electric field for $U_{c} \cdot U_{j}^{-1}=0$. The junction area is contained within the $x=-x_{j} . . x_{j}$ range.

Figure 6 shows plots of the electric field strength along the separation channel for different potential configurations. For the optimal splitting the field for $x>x_{j}$ should be positive, and for $x<-x_{j}$ negative. Under such conditions the fraction being split moves towards the right outlet, while the idle fraction is pulled to the left. In the case of the numerical model presented here these conditions are valid for $\mathrm{U}_{\mathrm{c}}=0.6 U_{j}$. However even then the contamination occurs, as shown in Figure 4, because of the presence of the diffusion-related spreading. To counterbalance also this, the $U_{c}$ must be lowered to the value of $0.28 U_{j}$ - only then no contamination takes place at $\Delta t=100 \mathrm{~ms}$. It must be noted that the pull-back voltage doesn't have to be present during the whole splitting process (i.e., during the time when voltage is 
applied to $U_{s p}$ ). In some situations such action may cause unnecessary repositioning of the fractions waiting in the separation channel. Often it is sufficient to apply the compensation voltage only for a fraction of the splitting time to move the idle components into a safe distance, where no electric field is present and diffusion has no effect.

\section{Experimental validation of forced splitting}

First, a standard CE separation of the sample mixture as specified in the materials section was performed with separation field strength $E_{S}=870 \mathrm{~V} \cdot \mathrm{cm}^{-1}$. Under these conditions the first component of the sample passed the detection point at $\Delta t=2.67 \mathrm{~s}$ after starting the separation. The total elution time of the components, measured between the front of the first peak and the tail of the last peak, was $\Delta t=3.16 \pm 0.06 \mathrm{~s}$. The average time gaps between the following fractions are given in Table 1 . The data was obtained by fitting electropherograms with a Gaussian function in OriginPro 8. Figure 7 shows a typical electropherogram registered by the detector positioned at the junction (three consecutive separations are shown). The time span between the fractions of rhodamine 110 and rhodamine 6G measures 280 ms (magnification in Figure 7) which renders the splitting procedure non-trivial.

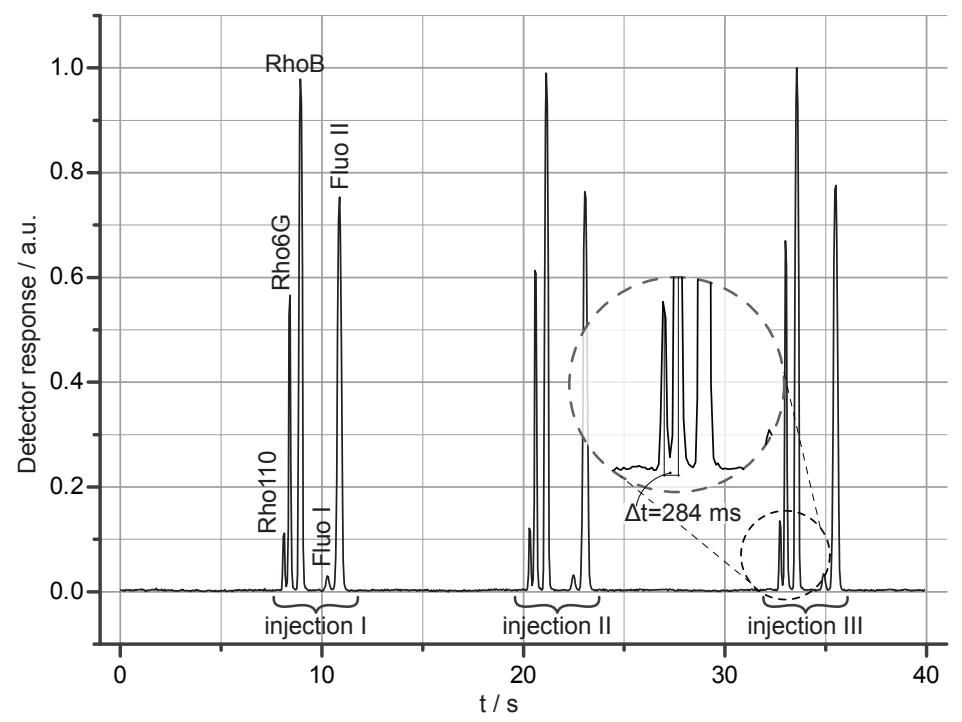

Figure 7. Electropherogram registered during standard CE separation of the sample mixture under a separation field strength $E_{s}=870 \mathrm{~V} \cdot \mathrm{cm}^{-1}$. (Three consecutive injections / separations are shown.) 
Table 1. Average time gaps between the peak maxima $\left(\Delta t_{\text {migration }}\right.$ denotes the time interval between the stated peak and the proceeding peak) and full-widthat-half-maximum (FWHM) of the peaks registered during normal sample separation. (Average values for 70 consecutive separations obtained by fitting electropherograms with a Gaussian function; for the rhodamine $\mathbf{1 1 0}$ fraction $\Delta t_{\text {migration }}$ is the time from the start of the separation.)

Rho. 110 Rho. 6G Rho. B Fluor.

\begin{tabular}{cllll}
\hline$\Delta \mathbf{t}_{\text {migration }}$ & 2.67 & 0.28 & 0.53 & 1.84 \\
$\mathbf{S}$ & \pm 0.36 & \pm 0.05 & \pm 0.06 & \pm 0.05 \\
$\mathbf{F W H M}$ & 0.103 & 0.116 & 0.194 & 0.223 \\
$\mathbf{S}$ & \pm 0.015 & \pm 0.013 & \pm 0.019 & \pm 0.015
\end{tabular}

Following a standard CE separation, automated splitting of the fractions was carried out. An electropherogram recorded during three consecutive separation/ splitting runs is shown in Figure 8.

The spacing between the fractions increased significantly. The preprogrammed durations of the splitting were: $\Delta \mathrm{tRho} 110=\Delta \mathrm{tRho6G}=2.6 \mathrm{~s}$ and $\Delta \mathrm{tRhoB}=2.8 \mathrm{~s}$. The inclusion of additional time gaps caused lengthening of the whole separation procedure - the total time between elution of the first and the last component was now $\Delta t=12.46 \pm 0.11 \mathrm{~s}$. The divisions of the fractions are clear and even the partly overlapping rhodamine 110 and rhodamine 6G fractions split properly.

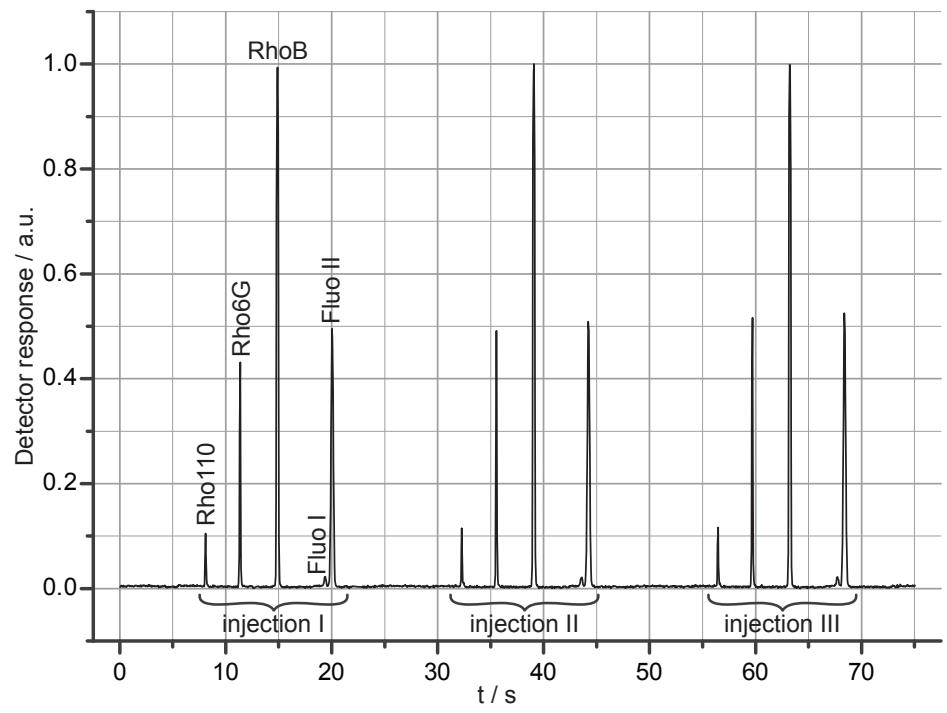

Figure 8. Electropherogram registered by the detector positioned over the junction during the CE separation with forced splitting of the fractions.

To test the time stability and the accuracy of the splitting procedure, we performed a series of separation tests. Each series consisted of 10 independent 
separation / splitting runs (50 runs in total). The interval between the series, during which the system was idle, was 5 minutes and the runs in series were performed with 10 seconds gaps.

Table 2 contains detailed data of calculated migration times and FWHM of the peaks. A detailed plot of FWHM vs. migration time difference for the first series is shown in Figure 9.

Table 2. Average time gaps between the peak maxima $\left(\Delta t_{\text {migration }}\right.$ denotes the time interval between the stated peak and the proceeding peak) and full-width-athalf-maximum (FWHM) of the peaks registered during sample separation with splitting. The mean values for five series, each consisting of 10 consecutive injections / separations, are shown.

\begin{tabular}{|c|c|c|c|c|c|c|}
\hline & & Series 1 & Series 2 & Series 3 & Series 4 & Series 5 \\
\hline \multirow{2}{*}{ 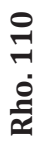 } & $\Delta t_{\text {migration }}$ & $\begin{array}{l}2.87 \\
\pm 0.02\end{array}$ & $\begin{array}{l}2.81 \\
\pm 0.05\end{array}$ & $\begin{array}{l}2.87 \\
\pm 0.07\end{array}$ & $\begin{array}{l}2.82 \\
\pm 0.04\end{array}$ & $\begin{array}{l}2.83 \\
\pm 0.04\end{array}$ \\
\hline & $\underset{S}{\text { FWHM }}$ & $\begin{array}{l}0.097 \\
\pm 0.006\end{array}$ & $\begin{array}{l}0.110 \\
\pm 0.016\end{array}$ & $\begin{array}{l}0.103 \\
\pm 0.004\end{array}$ & $\begin{array}{l}0.098 \\
\pm 0.005\end{array}$ & $\begin{array}{l}0.100 \\
\pm 0.006\end{array}$ \\
\hline \multirow{2}{*}{ 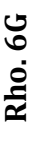 } & $\Delta t_{\text {migration }}$ & $\begin{array}{l}3.25 \\
\pm 0.01\end{array}$ & $\begin{array}{l}3.26 \\
\pm 0.03\end{array}$ & $\begin{array}{l}3.26 \\
\pm 0.02\end{array}$ & $\begin{array}{l}3.25 \\
\pm 0.02\end{array}$ & $\begin{array}{l}3.26 \\
\pm 0.02\end{array}$ \\
\hline & $\underset{S}{\text { FWHM }}$ & $\begin{array}{l}0.109 \\
\pm 0.002\end{array}$ & $\begin{array}{l}0.109 \\
\pm 0.002\end{array}$ & $\begin{array}{l}0.108 \\
\pm 0.004\end{array}$ & $\begin{array}{l}0.106 \\
\pm 0.001\end{array}$ & $\begin{array}{l}0.106 \\
\pm 0.004\end{array}$ \\
\hline \multirow{2}{*}{ 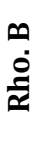 } & $\Delta t_{\text {migration }}$ & $\begin{array}{l}3.54 \\
\pm 0.02\end{array}$ & $\begin{array}{l}3.53 \\
\pm 0.02\end{array}$ & $\begin{array}{l}3.52 \\
\pm 0.02\end{array}$ & $\begin{array}{l}3.51 \\
\pm 0.01\end{array}$ & $\begin{array}{l}3.52 \\
\pm 0.02\end{array}$ \\
\hline & $\underset{\text { SW }}{\text { FWHM }}$ & $\begin{array}{l}0.206 \\
\pm 0.004\end{array}$ & $\begin{array}{l}0.205 \\
\pm 0.004\end{array}$ & $\begin{array}{l}0.202 \\
\pm 0.002\end{array}$ & $\begin{array}{l}0.199 \\
\pm 0.006\end{array}$ & $\begin{array}{l}0.197 \\
\pm 0.001\end{array}$ \\
\hline \multirow{2}{*}{$\stackrel{\ddot{\Xi}}{\stackrel{\Xi}{\Xi}}$} & $\Delta t_{\text {migration }}$ & $\begin{array}{l}5.15 \\
\pm 0.02\end{array}$ & $\begin{array}{l}5.14 \\
\pm 0.02\end{array}$ & $\begin{array}{l}5.14 \\
\pm 0.02\end{array}$ & $\begin{array}{l}5.09 \\
\pm 0.02\end{array}$ & $\begin{array}{l}5.06 \\
\pm 0.02\end{array}$ \\
\hline & $\underset{S}{\text { FWHM }}$ & $\begin{array}{l}0.245 \\
\pm 0.003\end{array}$ & $\begin{array}{l}0.247 \\
\pm 0.010\end{array}$ & $\begin{array}{l}0.240 \\
\pm 0.001\end{array}$ & $\begin{array}{l}0.238 \\
\pm 0.002\end{array}$ & $\begin{array}{l}0.235 \\
\pm 0.005\end{array}$ \\
\hline
\end{tabular}

The obtained results confirm very good accuracy of the method and repeatability of the procedure for 50 consecutive separation / splitting runs, during which the maximum average spread of the migration time differences was $26 \mathrm{~ms}$ (the data for the first fraction, rhodamine 110 , was not used for calculating this number, as the migration time of this component is not affected by splitting. 


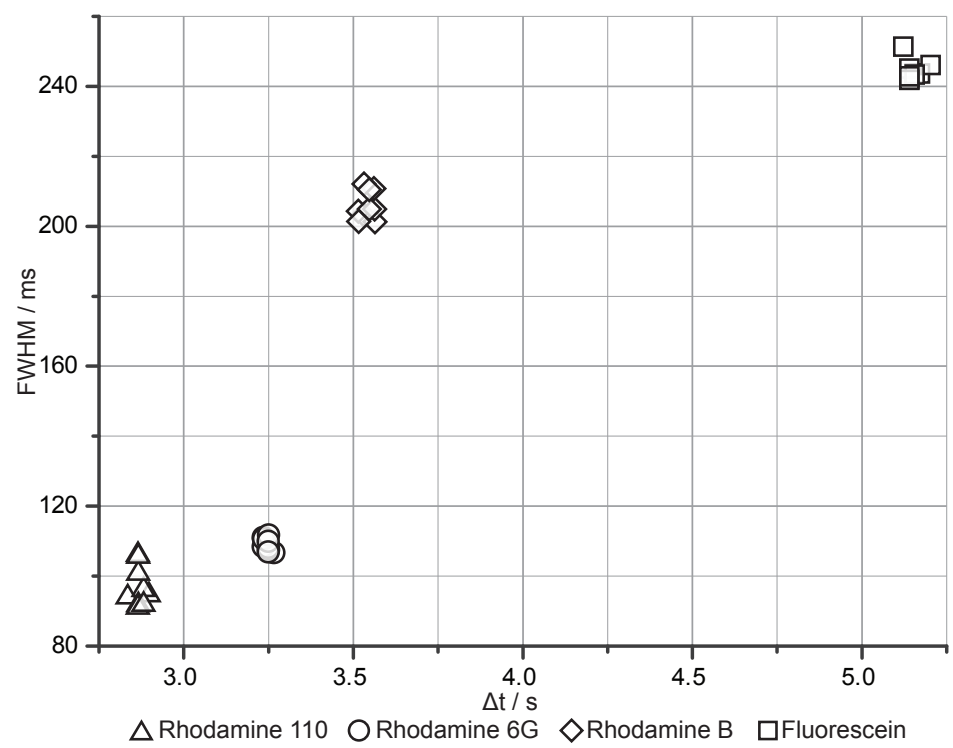

Figure 9. Scatter plot of observed peak widths vs. migration time for 10 consecutive separation-splitting runs.

Comparison of data obtained for normal separations (Table 1) and separations with forced splitting (Table 2) shows that the splitting procedure did not affect the width of detected peaks. However, the spacing between the peaks increased significantly. As a result the separation resolution enhanced. The average ratios of separation resolution calculated for electropherograms registered during separations with forced splitting and normal separations were: for rhodamine 110, rhodamine $6 \mathrm{G}$ peaks pair: $12.0 \pm 2.5$; for rhodamine $6 \mathrm{G}$, rhodamine B peaks pair: 6.7 \pm 0.9 ; and for rhodamine $B$, fluorescein peaks pair: $2.6 \pm 0.2$.

The splitting of fractions was performed with floating $U_{c}$ and it was found experimentally that no pull-back voltage was needed to prevent contamination (the idle fractions were positioned far enough from the junction during the splitting). However, to demonstrate the effect of undesired dragging of the waiting components during the splitting, we performed additional separations/ splitting during which the compensation voltage was applied. 


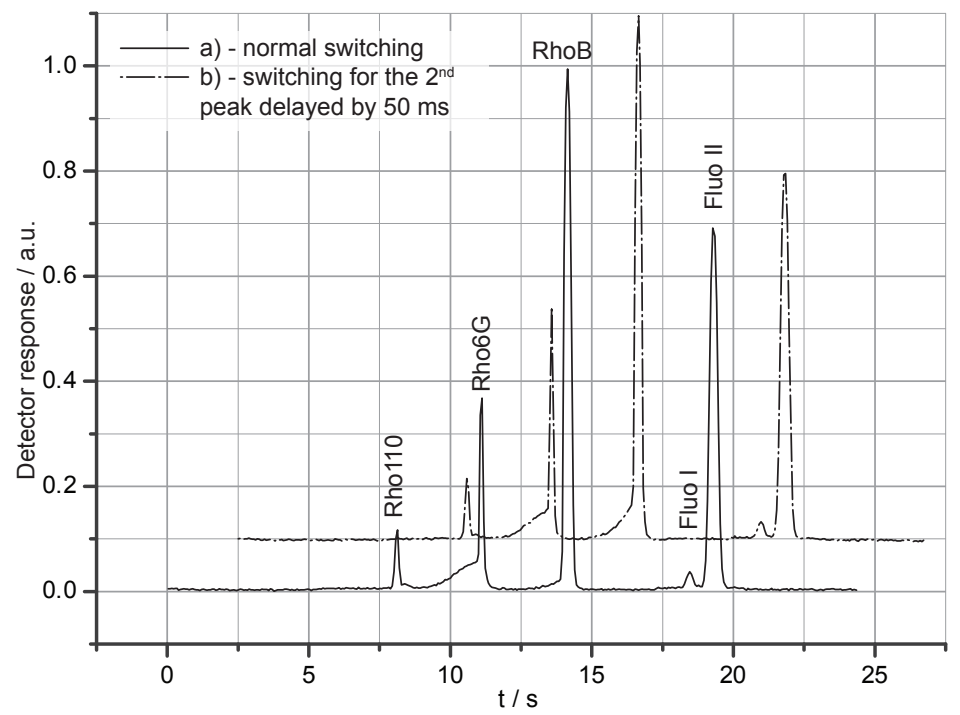

Figure 10. Electropherograms registered during splitting with improper voltage configuration $\left(U_{c}=1.05 \cdot U_{j}\right)$. Fronting of the rhodamine $6 \mathrm{G}$ fraction occurs on both electropherograms. Delaying the reaction of the system by $50 \mathrm{~ms}$ after detecting rhodamine $6 \mathrm{G}$ fraction (thus letting the idle fraction stop closer to the junction) results in fronting of the rhodamine B peak.

Figure 10 shows electropherograms recorded during splitting of the sample mixture with $U_{c}=1.05 \cdot U_{j}$. In both electropherograms an increase in the recorded intensity can be observed after the rhodamine 110 fraction passes the detector and splitting is performed. This fronting is caused by dragging the idle rhodamine 6G fraction out of the separation channel into the junction area, where the detector is located, and further downstream. This effect is almost not observed for the rhodamine $B$ peak in electropherogram a) in Figure 10, as the spacing between the rhodamine $6 \mathrm{G}$ and rhodamine $\mathrm{B}$ fractions $(\Delta t=529 \mathrm{~ms})$ is much greater than that between rhodamine 110 and rhodamine $6 \mathrm{G}$ fractions ( $\Delta t=250 \mathrm{~ms}$ ). Thus, during the splitting of the rhodamine $6 \mathrm{G}$ fraction, the rhodamine B peak resides far enough from the junction. However an increase of $50 \mathrm{~ms}$ in the separation time of rhodamine $6 \mathrm{G}$ (i.e. an inclusion of a pre-programmed delay in the splitting procedure, so that reconfiguration of voltages for splitting is delayed by $50 \mathrm{~ms}$ after detecting the rhodamine $6 \mathrm{G}$ peak) results in a significant difference. Since the reaction of the system was suspended, the rhodamine B fraction travelled further in the separation channel and stopped closer to the junction than in an unaffected experiment. As shown in electropherogram b) in Figure 10, this caused a similar fronting of the rhodamine B peak as of the rhodamine $6 \mathrm{G}$ peak. 


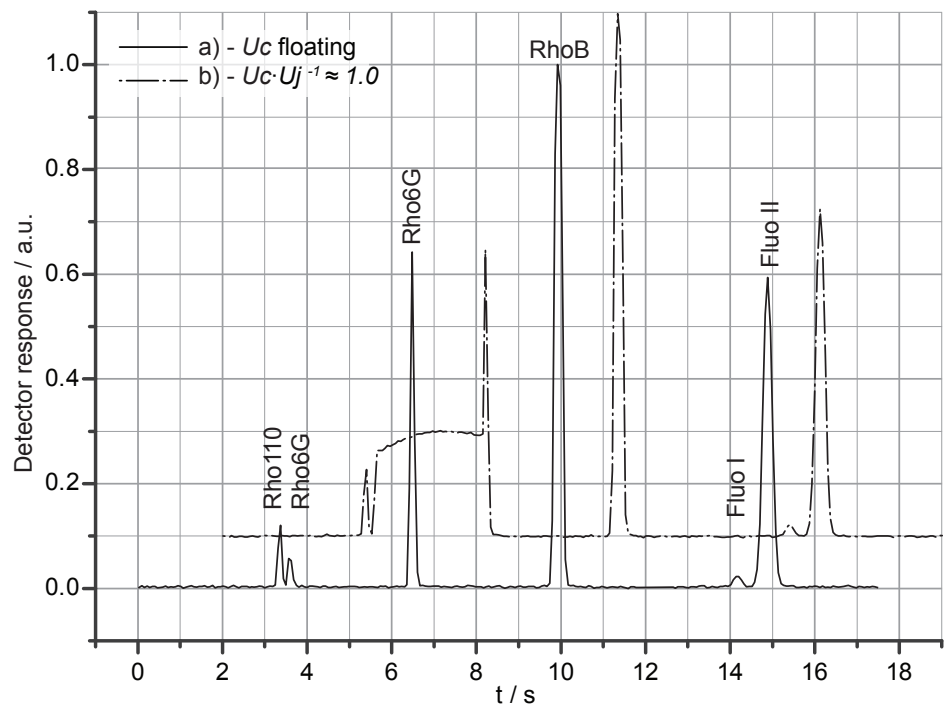

Figure 11. Electropherograms of splitting with hardware-related splitting error. A lag in the splitting procedure caused improper cutting of the rhodamine $6 \mathrm{G}$ fraction (a). In the case of a non-optimal potential configuration during splitting (b) such delay causes significant contamination.

The phenomenon of dragging the idle fractions can have a devastating effect when an error in splitting occurs. Figure 11 shows an electropherogram a) recorded during the splitting, when a hardware lag occurred and delayed the response of the system. As a result a part of the rhodamine 6G peak was cut and split together with the rhodamine 110 fraction. Such delayed reaction can cause significant contamination of the split component. The contamination is even larger when the lag occurs while the electric potential at the junction is not properly balanced. The electropherogram b) in Figure 11 shows such a situation for $U_{c}=1.01 U_{j}$ - a much greater portion of the rhodamine 110 fraction is dragged causing more contamination and undesired dilution of the sample. This experiment shows the importance of proper voltage configuration during splitting, which is in accordance with the numerical modelling results.

\section{Conclusions}

We proposed a method for performing precise spatial splitting of the adjacent fractions separated by CE in microfluidic format. Application of this technique in preparative CE devices may greatly simplify the manipulation of fractions, also reducing the risk of contamination between components. The significant spatial spacing, resulting from the use of this method, makes it possible to handle fractions individually in complex electrokinetically controlled channel networks. However, 
it is of great importance, to perform the splitting under pre-defined optimal conditions - doing otherwise may lead to undesired spreading, contamination and loss of analyte. It is also substantial, that the automation hardware used for the splitting performs reliably - even a difference of few tens of millisecond results in significant splitting errors. In the worst case faulty, hardware-error caused, splitting combined with operation under non-optimal conditions may result in significant contamination.

\section{References}

1. Huang, Y.F., C.C. Huang, C.C. Hu, and H.T. Chang, Capillary electrophoresis-based separation techniques for the analysis of proteins. Electrophoresis, 2006. 27(18): p. 3503-22.

2. Kasicka, V., Recent developments in capillary electrophoresis and capillary electrochromatography of peptides. Electrophoresis, 2006. 27(1): p. 142-175.

3. Kostal, V., J. Katzenmeyer, and E.A. Arriaga, Capillary Electrophoresis in Bioanalysis. Analytical Chemistry, 2008.

4. Kraly, J., M.A. Fazal, R.M. Schoenherr, R. Bonn, M.M. Harwood, E. Turner, M. Jones, and N.J. Dovichi, Bioanalytical applications of capillary electrophoresis. Analytical Chemistry, 2006. 78(12): p. 4097-4110.

5. Peng, Y.Y., A. Pallandre, N.T. Tran, and M. Taverna, Recent innovations in protein separation on microchips by electrophoretic methods. Electrophoresis, 2008. 29(1): p. 157-178.

6. Harrison, D.J., A. Manz, Z.H. Fan, H. Ludi, and H.M. Widmer, Capillary Electrophoresis and Sample Injection Systems Integrated on a Planar Glass Chip. Analytical Chemistry, 1992. 64(17): p. 1926-1932.

7. Manz, A., D.J. Harrison, E.M.J. Verpoorte, J.C. Fettinger, A. Paulus, H. Ludi, and H.M. Widmer, Planar Chips Technology for Miniaturization and Integration of Separation Techniques into Monitoring Systems - Capillary Electrophoresis on a Chip. Journal of Chromatography A, 1992. 593(1-2): p. 253-258.

8. Dolnik, V. and S. Liu, Applications of capillary electrophoresis on microchip. Journal of Separation Science, 2005. 28(15): p. 1994-2009.

9. Kim, M.S., S.I. Cho, K.N. Lee, and Y.K. Kim, Fabrication of microchip electrophoresis devices and effects of channel surface properties on separation efficiency. Sensors and Actuators B-Chemical, 2005. 107(2): p. 818-824. 
10. Tsai, C.H., R.J. Yang, C.H. Tai, and L.M. Fu, Numerical simulation of electrokinetic injection techniques in capillary electrophoresis microchips. Electrophoresis, 2005. 26(3): p. 674-686.

11. Bias, M., N. Delaunay, and J.L. Rocca, Electrokinetic-based injection modes for separative microsystems. Electrophoresis, 2008. 29(1): p. 20-32.

12. Matysik, F.M., Advances in amperometric and conductometric detection in capillary and chip-based electrophoresis. Microchimica Acta, 2008. 160(1-2): p. 1-14.

13. Guijt, R.M., C.J. Evenhuis, M. Macka, and P.R. Haddad, Conductivity detection for conventional and miniaturised capillary electrophoresis systems. Electrophoresis, 2004. 25(23-24): p. 4032-4057.

14. Viskari, P.J. and J.P. Landers, Unconventional detection methods for microfluidic devices. Electrophoresis, 2006. 27(9): p. 1797-1810.

15. Gotz, S. and U. Karst, Recent developments in optical detection methods for microchip separations. Analytical and Bioanalytical Chemistry, 2007. 387(1): p. 183-192.

16. Mogensen, K.B., H. Klank, and J.P. Kutter, Recent developments in detection for microfluidic systems. Electrophoresis, 2004. 25(21-22): p. 3498-3512.

17. Burns, M.A., B.N. Johnson, S.N. Brahmasandra, K. Handique, J.R. Webster, M. Krishnan, T.S. Sammarco, P.M. Man, D. Jones, D. Heldsinger, C.H. Mastrangelo, and D.T. Burke, An integrated nanoliter DNA analysis device. Science, 1998. 282(5388): p. 484-487.

18. Belder, D., M. Ludwig, L.W. Wang, and M.T. Reetz, Enantioselective catalysis and analysis on a chip. Angewandte Chemie-International Edition, 2006. 45(15): p. 2463-2466.

19. Lee, S.H., S.I. Cho, C.S. Lee, B.G. Kim, and Y.K. Kim, Microfluidic chip for biochemical reaction and electrophoretic separation by quantitative volume control. Sensors and Actuators B-Chemical, 2005. 110(1): p. 164-173.

20. Silvertand, L.H.H., E. Machtejevas, R. Hendriks, K.K. Unger, W.P. van Bennekom, and G.J. de Jong, Selective protein removal and desalting using microchip CE. Journal of Chromatography B, 2006. 839(1-2): p. 68-73.

21. Strausbauch, M.A. and P.J. Wettstein, Fraction Collection with Micro-Preparative Capillary Electrophoresis, in Handbook of capillary electrophoresis, J.P. Landers, Editor. 1997, CRC Press. p. 841-864.

22. Lin, R., D.T. Burke, and M.A. Burns, Selective extraction of size-fractioned DNA samples in microfabricated electrophoresis devices. Journal of Chromatography A, 2003. 1010 (2): p. 255-268. 
23. Sun, K., Z.Y. Li, K. Ueno, S. Juodkazis, S. Noji, and H. Misawa, Electrophoretic chip for high-fidelity fractionation of double-stranded DNA. Electrophoresis, 2007. 28(10): p. $1572-1578$.

24. Li, G., R. Ran, J.L. Zhao, and Y.S. Xu, Design, simulation, and optimization of a miniaturized device for size-fractioned DNA extraction. Electrophoresis, 2007. 28(24): p. 4661-4667.

25. Lerch, M.A. and S.C. Jacobson, Electrokinetic fluid control in two-dimensional planar microfluidic devices. Analytical Chemistry, 2007. 79(19): p. 7485-7491.

26. Tulock, J.J., M.A. Shannon, P.W. Bohn, and J.V. Sweedler, Microfluidic separation and gateable fraction collection for mass-limited samples. Analytical Chemistry, 2004. 76(21): p. 6419-6425.

27. Zalewski, D.R., S. Schlautmann, R.B.M. Schasfoort, and H.J.G.E. Gardeniers, Electrokinetic sorting and collection of fractions for preparative capillary electrophoresis on a chip. Lab on a Chip, 2008. 8(5): p. 801-809.

28. Khandurina, J., T. Chovan, and A. Guttman, Micropreparative fraction collection in microfluidic devices. Analytical Chemistry, 2002. 74(7): p. 1737-1740.

29. Effenhauser, C.S., A. Manz, and H.M. Widmer, Manipulation of Sample Fractions on a Capillary Electrophoresis Chip. Analytical Chemistry, 1995. 67(13): p. 2284-2287.

30. Kahle, V., V. Kost'al, and M. Zeisbergerova, Sample injection in capillary electrochromatography by heart-cut technique. Journal of Chromatography A, 2004. 1044(1-2): p. 259-265.

31. Killgore, J.K. and S.R. Villasenor, Systematic approach to generic matrix elimination via "heart-cut" column-switching techniques. Journal of Chromatography A, 1996. 739(1-2): p. 43-48.

32. Kadirvelu, K., C. Karthika, N. Vennilamani, and S. Pattabhi, Activated carbon from industrial solid waste as an adsorbent for the removal of Rhodamine-B from aqueous solution: Kinetic and equilibrium studies. Chemosphere, 2005. 60(8): p. $1009-1017$.

33. Mogensen, K.B., N.J. Petersen, J. Hubner, and J.P. Kutter, Monolithic integration of optical waveguides for absorbance detection in microfabricated electrophoresis devices. Electrophoresis, 2001. 22(18): p. 3930-3938.

34. Swinney, K. and D.J. Bornhop, Quantification and evaluation of Joule heating in onchip capillary electrophoresis. Electrophoresis, 2002. 23(4): p. 613-620.

35. Hu, L.G., J.D. Harrison, and J.H. Masliyah, Numerical model of electrokinetic flow for capillary electrophoresis. Journal of Colloid and Interface Science, 1999. 215(2): p. 300-312. 
36. $\mathrm{Wu}, \mathrm{C} . \mathrm{H}$. and R.J. Yang, Improvements on the electrokinetic injection technique for microfluidic chips. Electrophoresis, 2006. 27(24): p. 4970-4981. 


\section{Chapter V \\ Electrokinetic Sorting and Collection of Fractions for Preparative CE on a Chip}

$\int \begin{aligned} & \text { N THIS CHAPTER a microfabricated device capable of selecting } \\ & \text { and collecting multiple components from a mixture separated }\end{aligned}$ by capillary electrophoresis (CE) is described. This collection is automated and can be easily controlled by a set of rules defined by an operator, enabling fast and consistent operation. The device consists of an electrokinetically steered fluidic network that can be divided into three sections: a CE part, a fractions distribution region and a set of storage channels. Sample fractions leave the CE channel and are detected in the interfacial region by fluorescence intensity measurements. If an upcoming peak is detected, separation is withheld and the potentials are reconfigured to force the fraction into one of the collection channels, where they become available for further processing or analysis. The sequence of separation and collection is repeated until all the bands of interest are captured. A mixture of three fluorescent dyes (Rhodamine 6G, Rhodamine B and Fluorescein) was used to demonstrate the principle. The components were repeatedly separated by means of CE and pooled in their respective storage channels. In comparison to previous developments, the system presented in this paper offers automatic collection of all fractions in a single run. Furthermore, it is possible to run the system in a repetitive mode for accumulative pooling if more fractionated sample is required.

This chapter is published in Lab on a Chip, 2008, 8, 801-809. DOI: $10.1039 / b 717785 b$ 


\section{Introduction}

Since their introduction in the late 1970 s/early $1980 s,{ }^{1,2}$ capillary electrophoresis and its derivatives belong to the main analytical techniques in biological sciences. ${ }^{3-5}$ The potential of the method was demonstrated in the Human Genome Project ${ }^{6}$ and today CE serves as the dominant DNA sequencing method. New directions in biological research, such as proteomics, require improved analytical techniques and extensive post-separation sample processing. ${ }^{7,8}$ The development of effective procedures for the manipulation and collection of separated fractions is thus of increasing importance.

The first demonstration of fraction collection for capillary electrophoresis was performed by Hjerten and Zhu in $1985^{\circ}$ on nucleosides, $\mathrm{pH}$ markers and IEF ampholytes. In their system, sample was eluted from an open tubular capillary by buffer sheath flow and directed into a number of collection tubes. In the approach of Cohen et al,. collection of separated oligonucleotides was achieved by simply inserting the capillary together with an electrode into the tube containing buffer; ${ }^{10}$ slab gel electrophoresis was used subsequently to confirm the purity of a collected sample. This method was further extended by applying a field programming technique by Guttman and colleagues, ${ }^{11}$ which lowers the separation field during collection, enabling more precise operation.

The disadvantages of manual handling (high voltage hazard; inaccurate timing) were addressed by several groups, which concentrated on process automation. Rose and Jorgenson presented an apparatus that could perform preparative CE with collection into an array of vials by means of a capillary machine-moved at pre-programmed times. ${ }^{12}$ Lee et al. used an autosampler for collection of separated synthetic peptides. A critical aspect of timing was avoided by Huang and Zare who performed direct collection of amino acids onto a rotating drum using a frit structure for elution. ${ }^{13,14}$ In the following designs, fractionated proteins were collected onto a rotating circular membrane ${ }^{15}$ or onto a moving blotting membrane strip. ${ }^{16}$ In both cases, the membranes served as electrical interfaces between the end of the capillary and an electrode, and enabled easy post-processing of collected fractions by sequencing or immunological identification. More recently Minarik and co-workers designed a system for separating DNA fragments using 12 parallel separation capillaries and collection onto a moving microwell gel plate, the position of the fractions was reconstructed after analysis from the electropherograms and collected samples were subjected to PCR and sequencing. ${ }^{17}$ 
Muller et al. constructed a system capable of fully automated detector-triggered fractionation of DNA into a series of machine-switched collection capillaries. ${ }^{18} \mathrm{~A}$ similar concept was presented by Irie and co-workers who performed capillary array electrophoresis and collected the sample in individually addressed vial trays. ${ }^{19}$ The preparative mode of CE was also demonstrated on oligosaccharides using a sheath flow cell detector. ${ }^{20}$ This configuration was used in drug analysis in a simple preprogrammed manner. ${ }^{21}$

The concept of a modern micro total analysis system, introduced by A. Manz et al. in $1990,{ }^{22}$ together with a presentation of the first chip-based analytical separation device $^{23}$ led to the development of miniaturized electrophoretic systems in $1992 .^{24,25}$ Due to the limitations imposed by the nature of electrokinetic control and problems with force balancing at the microscale, most of the focus shifted towards improvement of the microchip CE rather than full system integration. Reports presenting CE as part of an integrated analytical microsystem ${ }^{26,27}$ are considered milestones and have drawn the attention of the scientific community. ${ }^{28}$

In the first microchip CE fractionator presented by Effenhauser et al,. ${ }^{29}$ the separation channel had two exits: one of them was used for collection while the other served as a waste channel. By alternating the applied potentials at predetermined times and closing an electric circuit, fractions could be drawn through either channel. Khandurina et al. performed micropreparative separation of DNA fragments in a simple crossed-channels device, employing manual reconfiguration of potentials in order to stop the separation and drive a single fraction into a reservoir for subsequent retrieval. ${ }^{30}$ In a device presented by Lin and colleagues sample fractions were redirected to the side channel at the T-junction and electrically captured downstream by integrated electrodes. ${ }^{31}$ Several difficulties concerning the peak dispersion that appeared during manipulation at the junction were later successfully addressed by introducing in-junction electrodes for local shaping of the electric field and thus minimizing undesirable effects. ${ }^{32}$ In the fully automated device described by Tulock et al., dispersion was avoided by utilizing a double layer channel system with polycarbonate track-etched membrane serving as fluidic isolation at the junction but still permitting forced fraction collection after reconfiguration of the steering potentials. ${ }^{33}$

Preparative CE cannot compete with the free-flow electrophoretic techniques if the amount of sample is significant or high-speed operation is required. However, it offers superior resolution, allows the use of capillary fillings and requires no 
pressure driven flow, so minimizing hardware requirements. A lack of hydraulic components usually implies minimization of dead-volume in the setup and thus reduces the amounts of both sample and analytes needed. A sample fractionated by means of preparative CE can be utilized in numerous ways including but not limited to subsequent analysis e.g. amino acid composition, sequencing, MS; or it can be used as a substrate for PCR, functional assays or labeling. ${ }^{34}$

In this paper, we report on the design and operation of a micropreparative CE chip capable of fast fractionation of multiple components from a complex sample. Following the separation, the sample fractions are sorted and stored in individually addressable collection channels, of which the number determines the number of unique collectable components. Real-time, dynamic computer control enables fast and time-stable operation and minimizes samples losses. Timing of the fractionation is triggered by the fluorescence intensity signal resolved by a photomultiplier at the end of the collection channel. Unlike with lab-scale instruments, the device incorporates no mechanical parts to assist the collection; the whole process is controlled electrokinetically. The method described in this report can be utilized as a primary or intermediate step in complex microfluidic systems. The on-a-chip integration of post-fractionation processing overcomes the difficulties with handling very small volumes and avoids the question of sufficiency of the limited amounts of product delivered by a single microscale separation. Lab-scale post-processing requires larger quantities of material. Sufficient amount of a sample can be provided by running the separation/collection procedure multiple times. Repetitive operation causes the accumulation of fractionated sample. As a result, the amount of collected material increases from femtomolar range - a usual product yield achieved in a single run for typical sample concentration - to picomols, a quantity sufficient for most analytical methods..$^{35-37}$

\section{Methods}

A schematic drawing of a micropreparative CE chip and a corresponding analyte concentration graph along the channel is shown in Figure 1. The chip geometry is described in terms of characteristic dimensions: separation channel length $-L_{S}$; collection channel length $-L_{C}$; and, for double-T injection - initial analyte plug width - $2 h$. The half-width $w$ of an analyte band is defined by its standard deviation $\sigma$ and equals: $3 \sigma$ and $h+3 \sigma$ for Gaussian and boxcar plug profiles respectively. It is also assumed that a method exists for manipulating the electric field independently in 
the separation and collection channels, i.e. performing separation while withholding collection and vice versa. This method will be discussed later.

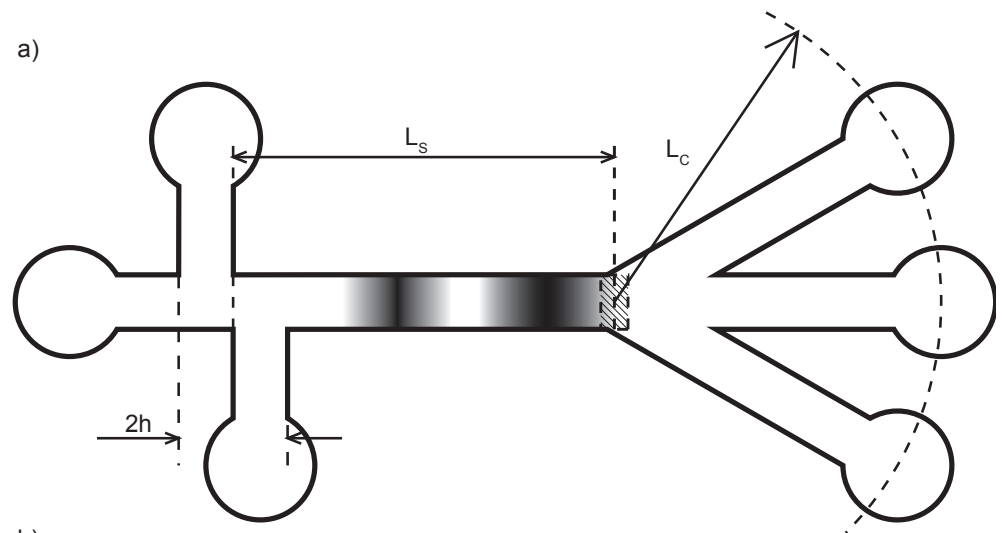

b)

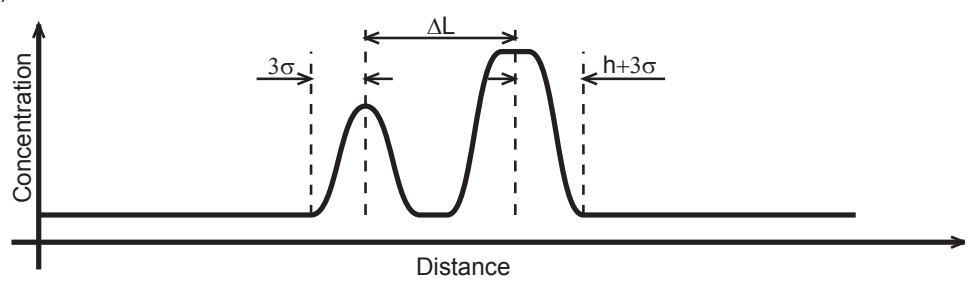

Figure 1. Schematic representation of a micropreparative CE chip device: a) characteristic dimensions; b) concentration profile of two plugs

\section{General system considerations}

Scaling laws of miniaturization ${ }^{38}$ i.e. reduction of distances and decrease of duration of processes as compared to traditional systems require more precise timing and higher spatial resolution of sample handling. Limits imposed by these requirements can be defined by a minimum operating frequency $f_{S}$ needed for an accurate spatial manipulation of fractions such that:

$$
f_{s}>\frac{u_{\max }}{\min \left(2 \cdot w_{\min }, \Delta L_{\min }\right)}
$$

where $u_{\max }$ is the maximum average velocity of a sample, $2 w_{\min }$ the minimum sample zone width and $\Delta L_{\min }$ the minimum distance between adjacent plugs. The feasibility of manipulation is usually determined by the full separation of the fractions:

$$
\Delta L_{\min }>2 \cdot w_{\min }
$$


For practical use in micropreparative CE systems, equation (1) combined with the assumption (2) can be written as:

$$
f_{s} \cdot t_{D 1}>\frac{1}{\gamma} \frac{L_{s}}{\sigma_{1}}
$$

where $t_{D 1}$ denotes the time needed for the most mobile fraction to reach the interface between the separation and collection parts, $\sigma_{1}^{2}$ is the variance of the fraction at time $t_{D 1}, L_{S}$ is a length of the separation channel and $\gamma$ is a parameter describing the desired spatial separation, e.g. for peaks with Gaussian concentration profiles it can be any number between 4 and 6 depending on the criterion used. Since $f_{S}$ usually depends on the hardware used and is limited by the computing speed and the response times of detectors and actuators, it cannot easily be altered. Thus, having fixed $f_{S}$, other parameters such as buffer composition, electric field strength and chip geometry need to be adjusted to obey the condition (3).

Information about the exact locations of fractions at any instant during separation and collection is an important prerequisite for the successful operation of micropreparative CE. In passive systems, ${ }^{29-31}$ such information is obtained from preliminary separations and an assumption is then made that the separation conditions instability' over time does not result in positional errors $\Delta s$ that lead to erroneous manipulation and sample misplacement, thus:

$$
\Delta s \ll \frac{u_{\max }}{f_{s}}
$$

Active systems ${ }^{33}$ collect their data during the separation through detection sites located at the channel and process it in order to reconstruct real-time information about the state of the process. In the simplest configuration, only one sensor is required at the interface between the separation and collection parts of the system. Usually a detector provides only data about the concentration profile of a passing band transformed by the detector's function. Based on this and given the position of an analyte at time $t_{0}$, the distance from an injection point to a detector $L_{s}$ and the electric field strength, the fraction properties such as mobility and diffusivity and its exact position can be calculated. Therefore on the assumptions that: a) external physical conditions are stable during a single run; b) electric field can be fully controlled and defined for any instant; and c) the system meets the minimum $f_{S}$ criterion; then this information can be later used for guiding the sample into an appropriate collection channel. Usually, active systems are able to deliver better 
results than passive devices and surpass later in adaptability. However, active systems are more complex than passive devices and require longer development times.

\section{Model of operation}

The performance of the device is strongly affected by the model of operation used internally to reconstruct sample parameters (mobility, diffusivity) and values of system variables (the operation's progress, positions of the fractions). If the conditions of time stability and definability of the potential distribution during the operation are met, the micropreparative CE device can be considered as a system that operates at three distinguishable electric field strengths: $E_{S}$ - the electric field of separation, that is field applied over separation channel length during the separation phase; $E_{C}$ - the electric field of collection, that is the field applied over the collection channel length during the collection phase and acting on the currently collected fraction, $E_{B}$ - the electric field applied over the separation channel during the collection phase and acting on the fractions that have not yet been collected. The electric field function of $\mathrm{i}$-th sample (that is the sample with the $i$-th greatest total mobility) is then given by:

$$
\begin{aligned}
E_{i}(t)= & E_{S} \cdot H(t)+ \\
& \left(E_{S}-E_{B}\right) \sum_{j=1}^{i-1}\left[H\left(t-t_{C j}\right)-H\left(t-t_{D j}\right)\right]+ \\
& \left(E_{C}-E_{S}\right) \cdot H\left(t-t_{D i}\right)-E_{C} \cdot H\left(t-t_{C i}\right)
\end{aligned}
$$

where $t_{D i}$, $t_{D j}$ is the time at which an $i$-th (or $j$-th) sample reaches the interface between the separation and collection parts of the system; $t_{C i}, t_{C j}$ - the time at which $i$-th (or $j$-th) sample reaches its final collection position and $H(\tau)$ is the Heaviside function. The times $t_{C}$ and $t_{D}$ can be obtained by applying following equations:

$$
\begin{aligned}
& t_{D i}=\left[\frac{\mu_{1}}{\mu_{i}}+\frac{\alpha}{\beta}(1-\eta) \sum_{j=1}^{i-1} \frac{\mu_{1}}{\mu_{j}}\right] \cdot t_{D 1} \\
& t_{C i}=\left[\frac{\mu_{1}}{\mu_{i}}\left(1+\frac{\alpha}{\beta}\right)+\frac{\alpha}{\beta}(1-\eta) \sum_{j=1}^{i-1} \frac{\mu_{1}}{\mu_{j}}\right] \cdot t_{D 1}
\end{aligned}
$$

where symbols $\alpha, \beta$ and $\eta$ are defined as: 


$$
\left\{\begin{array}{l}
L_{S}=L \\
L_{C}=\alpha L \\
E_{S}=E \\
E_{C}=\beta E \\
E_{B}=\eta E
\end{array}\right.
$$

and:

$$
t_{D 1}=\frac{L_{s}}{\mu_{1} \cdot E_{S}}
$$

The position of a sample at any instant can be determined by integrating equation (5) over time and then multiplying the result by sample mobility:

$$
x_{i}(t)=\mu_{i} \int_{0}^{t} E_{i}(\tau) d \tau
$$

The result of the integration is presented in Appendix section. The concentration profile of the sample is then given for Gaussian (11) and boxcar (12) plugs as:

$$
\begin{gathered}
C_{i}(x, t)=\frac{C_{0 i}}{\sigma_{i} \sqrt{2 \pi}} \exp \left(-\frac{\left(x-\mu_{i} \int_{0}^{t} E_{i}(\tau) d \tau\right)^{2}}{2 \sigma_{i}^{2}}\right) \\
C_{i}(x, t)=\frac{C_{0 i}}{2}\left[\begin{array}{c}
\operatorname{erf}\left(\frac{h-x+\mu_{i} \int_{0}^{t} E_{i}(\tau) d \tau}{2 \sqrt{D_{i} t}}\right)+ \\
\operatorname{erf}\left(\frac{h+x-\mu_{i} \int_{0}^{t} E_{i}(\tau) d \tau}{2 \sqrt{D_{i} t}}\right)
\end{array}\right]
\end{gathered}
$$

where: $\sigma_{i}^{2}=\sigma_{i n j}{ }^{2}+2 D_{i} t$ and $\sigma_{i n j}^{2}$ denotes the initial injection related variance of a plug.

It can be further related to the response of a detector by convolving (11), (12) with a detector response function. ${ }^{39}$ Applying the model equations to data obtained from detectors and known system parameters produces information needed for proper, dynamic control of the collection process. 


\section{Performance measures}

Many well established measures of the quality of separation exist such as resolution, plate height or plate number. ${ }^{39}$ Despite their widespread use they provide limited feedback when applied to preparative techniques, because they either depend on spatial separation as such, which in fraction collection is defined by system geometry, or because the quantities they define do not contain any information about the effectiveness of the collection process. As a consequence, the number of collectable fractions is introduced as an important performance metric.

\section{Number of collectable fractions}

The collection of two adjacent fractions is limited by the system's ability to recognize them at the interface between the separation and collection parts and to handle them independently. If the system operates at the minimum frequency $f_{S^{\prime}}$ this requirement can be easily met when there is no overlapping of the fractions:

$$
x_{i}(t)-x_{i+1}(t)>\gamma \cdot \frac{\sigma_{i}(t)+\sigma_{i+1}(t)}{2}
$$

Here $\gamma$ is an arbitrarily chosen number describing the quality of separation, e.g. for Gaussian plugs $\gamma=6$ defines a full baseline separation. On the assumption that the diffusivity of the analyte components is constant over the whole set, this general condition can be rewritten as:

$$
\begin{aligned}
& \frac{\mu_{i}-\mu_{i+1}}{\mu_{i}} \cdot L_{S}>\gamma \sqrt{\sigma_{i n j}^{2}+2 D t_{D i}} \\
& \frac{\mu_{i}-\mu_{i+1}}{\mu_{i}} \cdot L_{S}>\gamma\left(\frac{\sigma_{i n j}}{\sqrt{3}}+\sqrt{2 D t_{D i}}\right)
\end{aligned}
$$

for Gaussian (14) and boxcar (15) injection profiles respectively. The right side of equation (15) is the result of taking the integration limits for concentration profile function consistent with those for Gaussian plugs, i.e. such that for $\gamma=6$ (15) defines the full baseline separation. It is easy to show that, for any instant at least $99.7 \%$ of mass fraction of a boxcar concentration profile plug is contained within the range expressed below:

$$
\left(x_{i}-\left(h+3 \sigma_{D}\right), x_{i}+\left(h+3 \sigma_{D}\right)\right)
$$


For calculation of the number of collectable fractions a sample mixture that contains a limited number of components with equal diffusivities and mobilities $\mu_{i}$ that form an arithmetic series is defined as:

$$
\begin{aligned}
& \mu_{i}-\mu_{i+1}=\text { const }=\Delta \mu \\
& \mu_{i}=\mu_{1}-(i-1) \Delta \mu
\end{aligned}
$$

By using the definition of the dimensions (8) and applying (17) to (14) and (15), the collectability criterion can be reduced to a non-dimensional form given for Gaussian (18) and boxcar (19) injection profiles as follows:

$$
\begin{aligned}
& \frac{\varphi}{1-(i-1) \varphi} \sqrt{\frac{P e^{*}}{\chi P e^{*}+2 \zeta(i)}}>\gamma \\
& \frac{\varphi}{1-(i-1) \varphi} \frac{\sqrt{P e^{*}}}{\sqrt{\frac{\chi P e^{*}}{3}}+\sqrt{2 \zeta(i)}}>\gamma
\end{aligned}
$$

The $\zeta(i)$ is a function obtained from (6) and (17) that describes the relation between the collection time of the first fraction $t_{D 1}$ and $i$-th fraction $t_{D i}$ :

$$
t_{D i}=t_{D 1} \cdot \zeta(i)
$$

It is defined as:

$$
\begin{aligned}
& \zeta(i)=\zeta(i, \varphi, \alpha, \beta, \eta)= \\
& \left\{\begin{array}{cc}
1 & i=1 \\
\frac{1}{1-(i-1) \varphi}+\frac{\alpha}{\beta}(1-\eta) \sum_{j=0}^{i-2} \frac{1}{1-j \varphi} & i>1
\end{array}\right.
\end{aligned}
$$

The dimensionless parameters used in (18) and (19) are: $\varphi$ - dependent on the analyte mixture composition, $P e^{*}$ - Péclet number related to the separation path length and $\chi$ - a number characterizing the initial plug variance related to the square of separation path length. 


$$
\left\{\begin{array}{l}
\varphi=\frac{\Delta \mu}{\mu_{1}} \\
P e^{*}=\frac{\mu_{1} E L}{D} \\
\chi=\frac{\sigma_{i n j}^{2}}{L^{2}}
\end{array}\right.
$$

Equation (19) can be rewritten in a form closely resembling that of (18):

$$
\frac{\varphi}{1-(i-1) \varphi} \Lambda(\vartheta) \sqrt{\frac{P e^{*}}{\chi P e^{*}+2 \zeta(i)}}>\gamma
$$

where $\Lambda(\vartheta)$ given by:

$$
\left\{\begin{array}{l}
\Lambda(\vartheta)=\frac{\sqrt{3+\vartheta}}{1+\sqrt{\vartheta}} \\
\vartheta=\frac{6 \zeta(i)}{\chi P e^{*}}
\end{array}\right.
$$

It can be seen that for large $\vartheta$ (i.e. long time, small initial peak widths, high diffusion coefficient, long separation channels, thus everything that makes the diffusional dispersion significant) $\Lambda$ (७) tends to 1 and (19) is reduced to the form given for Gaussian plugs equation (18). In further analysis, equation (23) will be used as the main collectability criterion with $\Lambda(\vartheta)=1$ for Gaussian plugs and $\Lambda$ ( $७)$ defined by (24) for boxcar plugs.

The number of theoretically collectable fractions can be obtained by calculating the first positive root $i$ of a function:

$$
f\left(i, \varphi, P e^{*}, \chi, \alpha, \beta, \zeta\right)=\frac{\varphi}{1-(i-1) \varphi} \Lambda(\vartheta) \sqrt{\frac{P e^{*}}{\chi P e^{*}+2 \zeta(i)}}-\gamma
$$

Solving this problem analytically is not trivial, but the solution can also be obtained by a numerical search for the smallest $\mathrm{n}$ such that:

$$
\begin{aligned}
& n \in N:(\left.f\left(n, \varphi, P e^{*}, \chi, \alpha, \beta, \zeta\right)>0\right) \wedge \\
&\left(f\left(n+1, \varphi, P e^{*}, \chi, \alpha, \beta, \zeta\right) \leq 0\right)
\end{aligned}
$$

If found, $n$ denotes the maximum number of theoretically collectable fractions from a sample mixture with properties defined in (17). 

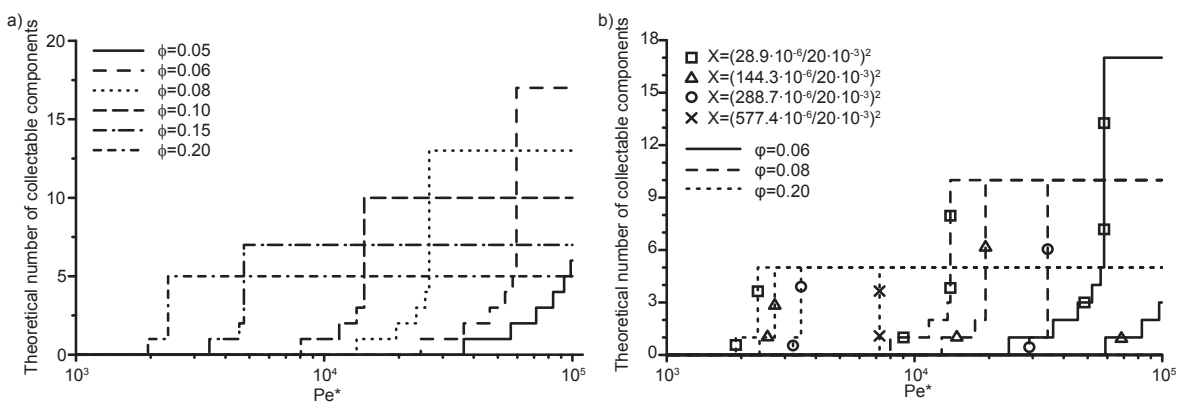

Figure 2. Graphs of calculated theoretical number of collectable components for: a) varying $\varphi$ and b) varying $\chi$; for boxcar injection concentration profile.

In Figure 2, the results of calculation of the theoretical number of collectable components for boxcar injection profile are shown. Parameters used in calculations were: $\alpha / \beta=1 / 1.5, \eta=0$ and additionally in Figure $2 \mathrm{a} \chi=\left(\left(28.9 \cdot 10^{-6}\right) / 2 \cdot 10^{-2}\right)^{2}$ (plug length $2 h=100 \mu \mathrm{m})$. It is clear that for large $P e^{*}$, the number of collectable fractions is limited only by the analyte composition, i.e. all the fractions can be collected, whereas for lower $P e^{*}$ values the collection is diffusion-limited, the transition region width depends on the value of $\varphi$. It can be also noted that increasing the value of $\chi$ greatly decreases the number of collectable components for a given $P e^{*}$. This effect is most noticeable in complex samples. It is an important design consideration as, depending on other parameters and needs, it has to be decided whether to increase throughput by maximizing the injection volume or to focus on the system flexibility and its possible application in multicomponent sample analysis. Additionally it must be noted, that however the number of theoretically collectible components is relatively large, in practice it is limited by the number of collection channels. To accommodate for larger quantity of fractions to be collected, the chip design must incorporate corresponding number of supplementary collection channels.

\section{Experimental section}

\section{Materials}

Rhodamine B, rhodamine 6G, fluorescein, 2-(N-morpholino)ethanesulfonic acid (MES), histidine, $\mathrm{NaOH}$ and isopropyl alcohol (IPA) were obtained from SigmaAldrich. Stock solutions of $10 \mathrm{mg} / \mathrm{mL}$ of rhodamine B, rhodamine $6 \mathrm{G}, 6 \mathrm{mg} / \mathrm{mL}$ of fluorescein, $100 \mathrm{mM}$ MES and histidine were prepared in demineralised water. Prior to experiments, stock solutions were used to prepare $20 \mathrm{mM}$ MES/histidine buffer at $\mathrm{pH} 6.35$ and $0.1 \mathrm{mM}$ rhodamine $\mathrm{B}$, rhodamine $6 \mathrm{G}$, fluorescein in $20 \mathrm{mM}$ MES/ 
histidine sample solution. All mixtures were filtered with $0.22 \mu \mathrm{m}$ membrane filters and degassed in vacuum for 30 minutes before transferring onto a chip.

\section{Device fabrication}

A photograph of the chip is shown in Figure 3. The fluidic network was filled with a red dye for visualization. The conductivity detector, of which electrodes are marked with symbol ' $D$ ' on the picture, was not used in experiments.
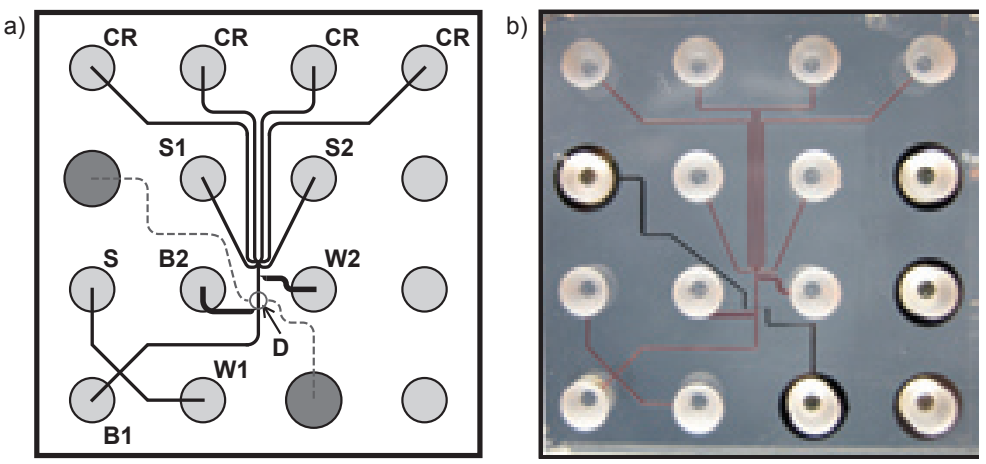

Figure 3. Photograph of a chip with visible fluidic network.

The chip consists of two bonded $1.1 \mathrm{~mm}$ thick borosilicate glass plates. The top plate contains the fluidic channels as well as reservoir openings. To create the fluidic channels, a $10 \mathrm{~nm}$ chromium adhesion layer followed by a $140 \mathrm{~nm}$ gold layer was sputtered on a $100 \mathrm{~mm}$ glass wafer (Schott Borofloat 33). The $\mathrm{Cr} /$ $\mathrm{Au}$ layer is resistant to hydrofluoric acid and acts as a mask during wet-etching of the channels. The transfer of the fluidic network design into the $\mathrm{Cr} / \mathrm{Au}$ mask was carried out by photolithography with Olin 907/17 photoresist, followed by removal of gold and chromium in the exposed areas by wet-etching. Subsequently, a $10 \%$ solution of hydrofluoric acid was used to etch $10 \mu \mathrm{m}$ deep channels into the glass. The reservoir openings were fabricated by micro-powderblasting with $29 \mu \mathrm{m} \mathrm{Al}_{2} \mathrm{O}_{3}$ particles through a patterned $100 \mu \mathrm{m}$ thick polymer photoresist foil as described by Wensink et $a l^{40}$ After stripping of the photoresist layers in acetone and removal of the $\mathrm{Cr} / \mathrm{Au}$ mask by wet-etching, the top and bottom glass wafers were cleaned in $100 \% \mathrm{HNO}_{3}$ for $15 \mathrm{~min}$., followed by rinsing and dry spinning. Next the wafers were brought into close contact to obtain a pre-bond. Light pressure was applied to spread the pre-bonded area across the entire wafer. Irreversible bonding was achieved by annealing at $600{ }^{\circ} \mathrm{C}$ for one hour. Finally, the bonded wafer stack was diced into separate $20 \mathrm{~mm} \times 20 \mathrm{~mm}$ chip devices. 


\section{Instrumentation}

A custom-made holder was used to provide electrical and fluidic connections to the chip. Three high-voltage, four-channels power supplies (CU 411, IBIS Technologies, Hengelo, the Netherlands) served as voltage sources. The fluorescence intensity measured by a photomultiplier tube at the point marked with symbol ' $D$ ' in Figure 3 was used for fractions detection. The amplified detector signal was acquired with a multimeter (Agilent 34401A). Utilization of a digital multimeter with an independent detector allows for use of other detection methods employed commonly in CE. The instruments were controlled by a native Windows ${ }^{\mathrm{TM}}$ application, written in-house, with a time resolution of at least $40 \mathrm{~ms}$. An inverted microscope (Olympus IX51) equipped with a mercury burner, fluorescent filter set (XF57, Omega Optical, USA) and a 32 bit colour CCD camera (ColorView II, Olympus) controlled by Analysis software package (Olympus Soft Imaging Solutions) was used for visualization.

\section{Chip operation}

For a description of the operation of fractionation, we refer to the symbols in Figure 3. The operation starts after submitting a steering script that contains injection and separation fields, injection time, sheath flow magnitudes, collection velocities and a set of fractions selected for collection, all to the control application. First, a quick flashing procedure is performed by forcing buffer flow between B1 and $\mathrm{W} 2$, then between B2 and CR, and finally between S1, S2 and CR reservoirs. Subsequently a buffer is allowed to flow from B1 to W2 under pre-defined separation conditions and the data for establishing background fluorescence magnitude is collected. The separation starts with an injection of a sample into the separation channel by applying voltages to sample reservoir (S) and waste (W1). Next, the voltages are switched to establish a separation field by applying a potential difference between the buffer source (B1) and the waste (W2). On the decision of an operator, a pre-fractionation run can be made at this point. This step is completely optional and meant only for choosing the fractions to be collected or checking the separation conditions. If the pre-fractionation is omitted, the operator must know which fractions should be collected in advance or let the system collect the components in arrival order. The pre-fractionation is achieved by allowing all the fractions to go to the waste (W2) passing the detector; an electropherogram recorded during this step and mobilities calculated from it are used in the subsequent runs as a base for fractionation. Alternatively, a standard separation/collection cycle is performed. The collection procedure is triggered by threshholding the detector signal. The separation field is kept until a fraction passes the detection point, next the potentials 
are reconfigured; separation is withheld and in the case of a fraction pre-selected for waste, the voltages are applied to buffer source (B2) and waste (W2) forcing the sample to enter waste reservoir W2. In the case of collection, migration of a sample is forced between B2 and one of the collection channels (CR). Additionally a sheath flow from S1 and S2 is switched on by applying appropriate voltages, resulting in electrokinetic focusing of a sample to assist the collection and prevent diffusional cross-contamination of the collection channels. During the collection, an electric field distribution is established, based on electrophoretic mobility of a fraction calculated from a recorded migration time and a desired collection velocity value provided by an operator. In the case of accumulative collection, when fractions already reside in the storage channels, at least one of these channels must be used as a waste sink for a buffer volume preceding the sample being collected. Directing unwanted buffer to the waste channel prevents dilution of the pooled fractions and possible contamination.

\section{Results and discussion}

\section{Fraction collection and accumulation}

Three fluorescent standards were used to demonstrate collection of a CE separated sample. Figure 4 a shows a composite image of collection channels after a series of 30 consecutive full separation/collection cycles. The device was operated in an automated fashion with only three field strengths $\left(E_{S}=750 \mathrm{~V} \cdot \mathrm{cm}^{-1}, E_{C}=550 \mathrm{~V} \cdot \mathrm{cm}^{-1}\right.$, $\left.E_{i n j}=1250 \mathrm{~V} \cdot \mathrm{cm}^{-1}\right)$ and the injection time $\left(t_{i n j}=3 \mathrm{~s}\right)$ defined in a steering script. Three channels containing separated sample fractions are visible; there is also a waste channel, which cannot be clearly distinguished due to very low fluorescent dyes concentration within it. Fluorescence intensity profiles measured along all four collection channels are shown in Figure 4b. Gaussian-like peaks can be observed in the channels used for collection, with broader and lower peaks to the right due to their longer residence in the channels and thus greater diffusional dispersion. The magnitude of measured fluorescence of the waste channel is significantly lower as compared to the other channels; a slight increase of intensity can, however, be noticed as the position along the channel progresses. The purity of the collected fractions and the nature of the contamination in the waste channel were assessed by mapping the fluorescence image into the sRGB colour space. 


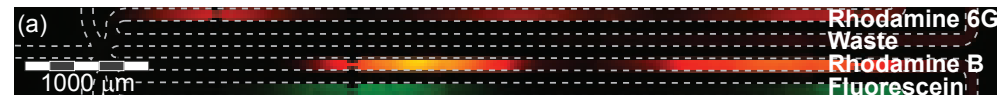

(b)

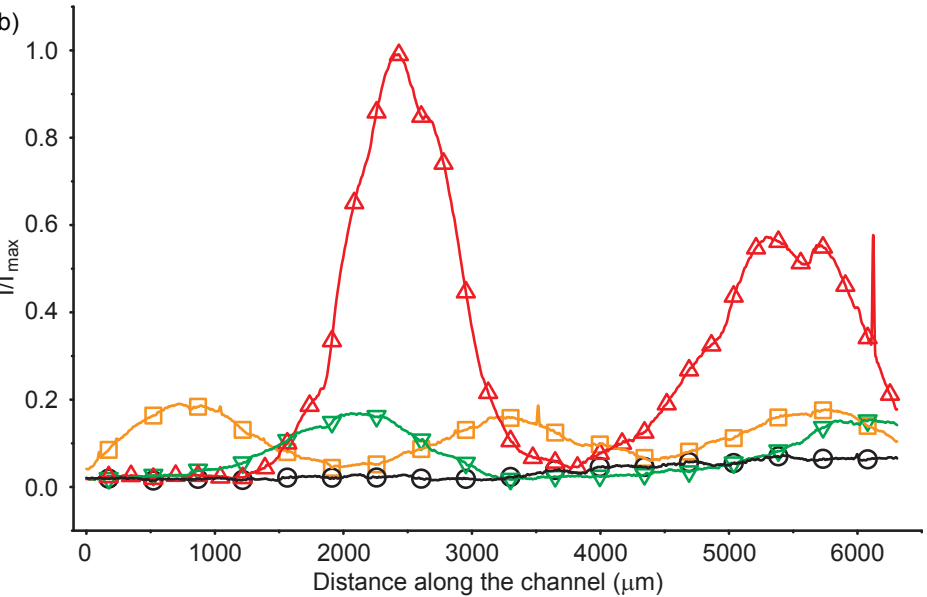

$\square$ Rhodamine 6G $\odot$ Waste (empty) $\triangle$ Rhodamine B $\nabla$ Fluorescein (c)
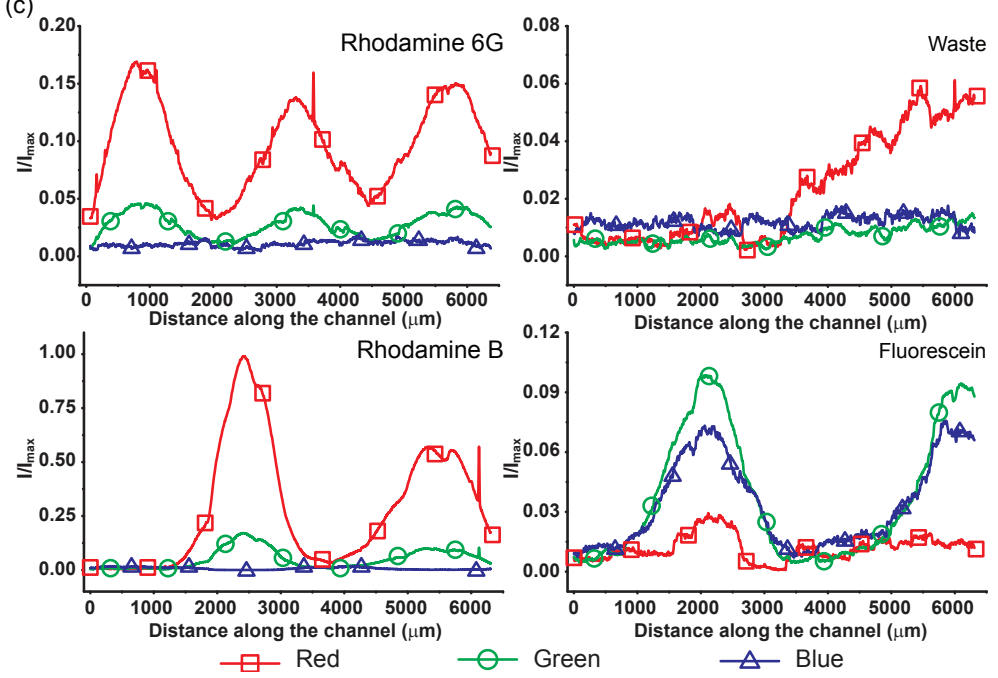

Figure 4. Results of separation/collection of fluorescent standards; a) composite fluorescence image of collection channels with stored fractions (brightness has been enhanced for visualization); b) fluorescence intensity graphs along the channels; c) intensity profiles of primary colours in the SRGB colour space along the channels.

Figure 4c shows the intensity plots of the primary colours of the sRGB colour space (red, green, blue). The differences between the plots of the fractions are clearly visible. The most pronounced colour component in rhodamine B and rhodamine 6G, red, is not noticeable in fluorescein, also blue, plainly visible in fluorescein, is virtually absent from both rhodamines. The fluorescence observed 
in the waste channel is most likely a result of contamination by either rhodamine B or rhodamine 6G; this conclusion can be drawn from the sRGB intensity profiles and the location of the impurity.

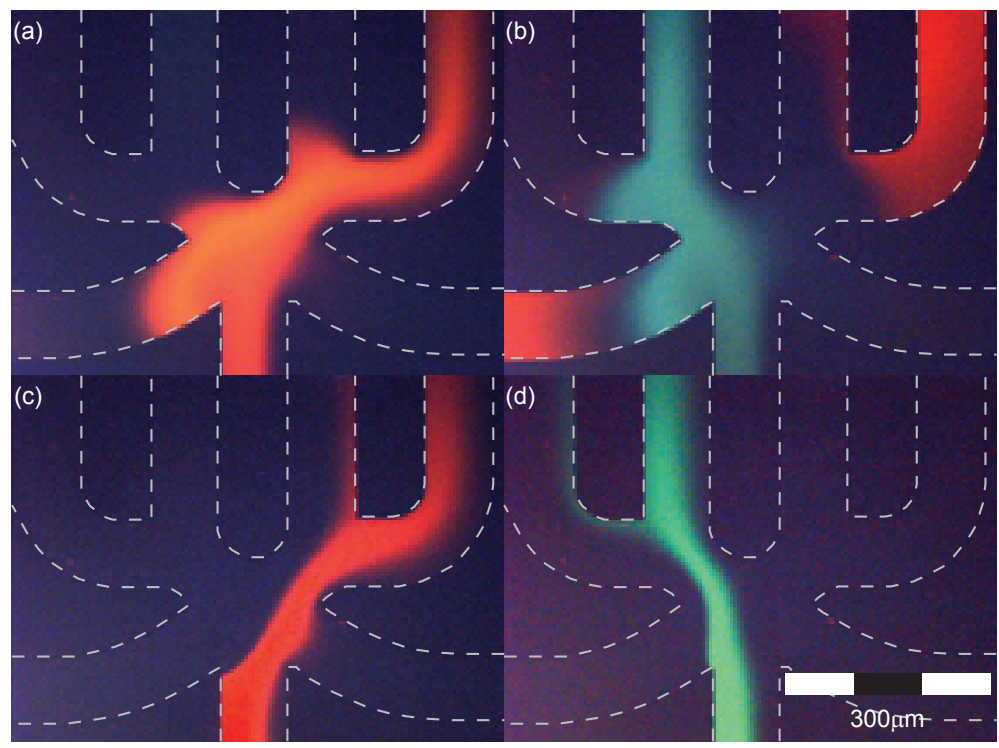

Figure 5. Fractions guiding: rhodamine B entering the 4th collection channel a) without electrokinetic focusing, c) with electrokinetic focusing; fluorescein entering the 2nd collection channel after rhodamine B has been stored: b) without electrokinetic focusing, d) with electrokinetic focusing.

The cross-contamination between adjacent channels, seen in the waste channel, although very small, is almost impossible to avoid; this is due to the lack of techniques for incorporating adjustable mechanical barriers into electrokinetically operated device, drag force acting on steady fluid when in contact with moving stream and penetration of the electric field into floating side channels at the crossings. The results of the final phenomena can be minimized to some extent during the collection by utilizing electrokinetic focusing of the sample stream, as shown in Figure 5. The unwanted spreading of fractions demonstrated in Figure 5a,b is easily avoided as shown in Figure 5c,d; the fill coefficient of the focusing (i.e. a fraction of the stream width occupied by a sample) was $85 \%$. A visible, non-significant flow of parts of the fractions to the left-sided adjacent collection channels is caused by an unbalanced leak from the waste channel W2.

\section{Overlapping fractions}

In some scenarios, it may be difficult to achieve full electrophoretic separation of adjacent fractions. However, it is still advantageous to collect them individually 
even if some contamination is unavoidable. Those situations can be addressed by a technique of forced electrokinetic splitting of overlapping components.
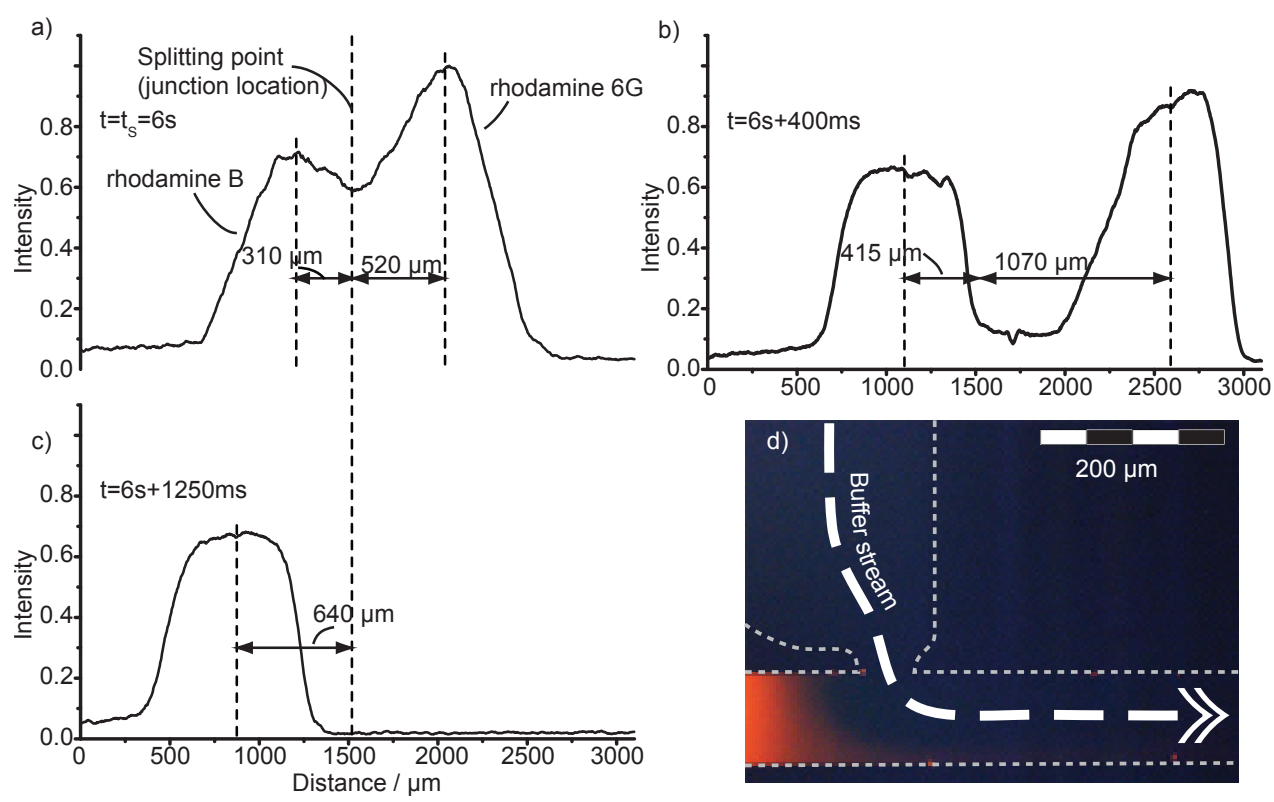

Figure 6. Forced splitting of overlapping fractions of rhodamine B and rhodamine $6 \mathrm{G}$. Fluorescence intensity profiles taken after a) 0 ms, b) $400 \mathrm{~ms}$, c) $1250 \mathrm{~ms}$ from the beginning of operation; $d$ ) - fluorescence image of the front of rhodamine B peak at the junction.

For performing forced division, the fractions must be positioned at a crossing (or a T-junction) in such a way that the minimum between the peaks is located exactly in the middle of a junction. At this point, the potentials are reconfigured so that the flow is forced from the side channel into the channel that contains the sample. This results in fractions being pushed aside and forced to separate. Such a scheme may be also useful in electrokinetic column coupling, e.g. in the case of blood analysis by $\mathrm{CE}$, where a low-abundant peak of $\mathrm{Li}^{+}$is cut off from a large concentration $\mathrm{Na}^{+}$zone. ${ }^{41}$ To demonstrate application of this technique in preparative CE, a separation of a mixture of rhodamine B/ rhodamine 6G was performed. The injection plug width was $500 \mu \mathrm{m}$ and the separation and collection fields were both lowered to around $375 \mathrm{~V} \cdot \mathrm{cm}^{-1}$; performing separation under these conditions resulted in overlapping of the rhodamines' fractions at the arrival to the detection point. The fluorescence intensity profiles taken during the consecutive steps of artificial splitting are show in Figure 6a-c. A clear division of the peaks can be seen as well as distortion of their shapes due to the applied conditions. A microscopic fluorescent picture of 
rhodamine B fraction front at the junction taken around $400 \mathrm{~ms}$ after splitting is shown in Figure 6d.

\section{Time stability and repeatability}

The reproducibility of the separation/collection process was tested in two series of experiments with 30 minutes in between. Each series consisted of ten full runs separated by 30 second breaks; six middle experiments in each series were utilized for measurements. The positions and standard deviations of the fractions were determined by least-square fitting to the Gaussian function (11) of the intensity profiles computed from the fluorescence images taken directly after a collection of each fraction. As seen in Figure 7, the reproducibility of collection between the series and the runs is very good. The fractions were pre-programmed to stop at the distance of $2000 \mu \mathrm{m}$ (rhodamine B, 0.33 of the length of collection channels) and $1500 \mu \mathrm{m}$ (fluorescein, 0.25 of the length of collection channel). Misplacement of the samples falls into the theoretical limits defined by the operating frequency (4); with $u_{\max }=3100 \mu \mathrm{m} \cdot \mathrm{s}^{-1}$ and $f_{s}=25 \mathrm{~Hz}$ the maximum theoretical positional error is $\Delta s=124 \mu \mathrm{m}$, that is 0.021 of the length of collection channels.

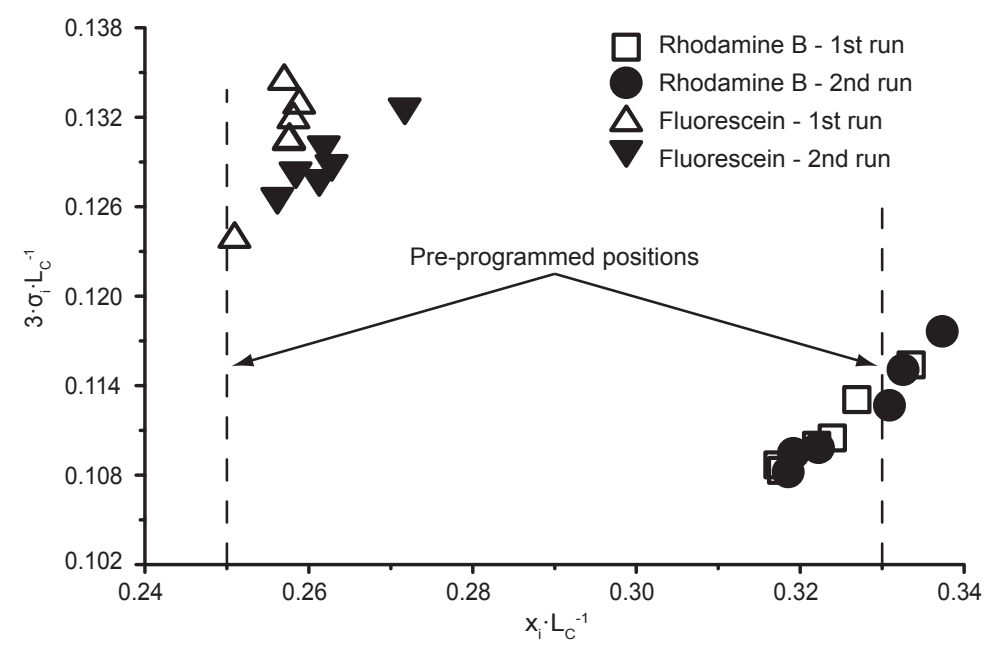

Figure 7. Reproducibility of the operation. Positions in collection channels versus half-widths of peaks directly after storing for two consecutive series are plotted.

\section{Conclusions}

A method was proposed for performing micro-preparative CE on a chip that allows for the accumulative pooling of all fractions. Furthermore, the model of 
operation and a set of parameters were introduced, resulting in optimization guidelines and considerations for the setup and chip design.

The system based on the described principles was built and microfluidic preparative capillary electrophoresis was successfully performed in an automated fashion on a sample mixture. The decoupling of separation and collection processes, realized by individual addressing of both sections of the chip, enabled collection of all the components in a single run and pooling of identical fractions in tight series. Also, the application of the electrokinetic focusing for prevention of cross-contamination between adjacent channels in fluidic manifolds was demonstrated, with very good results. By utilizing active, real-time computer control of the operation excellent reproducibility and time stability of the operation was achieved. It was also shown that automatic control combined with a proper fluidic network design allows for 'mechanical' splitting of overlapping fractions separated by CE. The techniques, described in this paper, for fast and precise electrokinetic manipulation of individual sample zones in aqueous solution may also be applicable in other systems, e.g. multidimensional separation, accurate sample delivery and weighting for labelling or reaction studies, positioning for local surface-dependent studies or localized measurements.

\section{Appendix}

The result of calculating the integral (10)

$$
x_{i}(t)=\mu_{i}\left\{\begin{array}{l}
E_{S} \cdot t \cdot H(t)+ \\
\left(E_{S}-E_{B}\right) \sum_{j=1}^{i-1}\left[\left(t-t_{C j}\right) \cdot H\left(t-t_{C j}\right)-\left(t-t_{D j}\right) \cdot H\left(t-t_{D j}\right)\right]+ \\
\left(E_{C}-E_{S}\right)\left(t-t_{D i}\right) \cdot H\left(t-t_{D i}\right)-E_{C}\left(t-t_{C i}\right) \cdot H\left(t-t_{C i}\right)
\end{array}\right\}
$$

\section{Symbols}

$L_{S}$ - separation channel length

$L_{C}-$ collection channel length

$2 h$ - initial analyte plug width for double-T injection

$\sigma-$ peak spatial standard deviation

$f_{S}-$ minimum operating frequency of a system

$\mathrm{u}_{\max }-$ maximum average velocity of a sample 
$2 w_{\text {min }}$ - minimum sample zone width

$\Delta L_{\text {min }}-$ minimum distance between adjacent plugs

$t_{D 1}$ - time needed for the most mobile fraction to reach a detector

$\gamma-$ parameter describing the desired spatial separation

$\Delta s$ - positional error

$E_{S}-$ electric field strength during separation

$E_{C}-$ electric field strength during collection

$E_{B}$ - electric field applied over a separation channel during the collection phase

$t_{D i}-$ time at which an $i$-th sample reaches an interface between the separation and collection parts

$t_{C i}-$ time at which an $i$-th sample reaches its final collection position

$H(\tau)$ - the Heaviside function

$\alpha$ - ratio between $L_{C}$ and $L_{S}$

$\beta$ - ratio between $E_{C}$ and $E_{S}$

$\eta$ - ratio between $E_{B}$ and $E_{S}$

$x_{i}(t)$ - position of an $i$-th sample at time $t$

$E_{i}(t)$ - electric field function of an $i$-th sample

$\mu_{i}-$ mobility of an $i$-th sample

$t$ - time

$C_{o i}$ - initial sample concentration

$\sigma_{i n j}^{2}-$ initial injection spatial variance of a plug

$D_{i}$ - diffusion coefficient of an $i$-th sample

$\Delta \mu$ - average difference between mobilities of adjacent plugs

$\varphi-$ ratio of $\Delta \mu$ and mobility of the most mobile plug

$P e^{*}$ - Péclet number related to the separation path length

$\chi$ - number characterizing the initial plug variance related to the square of separation path length.

\section{References}

1. Mikkers, F.E.P., F.M. Everaerts, and T. Verheggen, High-Performance Zone Electrophoresis. Journal of Chromatography, 1979. 169(FEB): p. 11-20. 
2. Jorgenson, J.W. and K.D. Lukacs, Zone Electrophoresis in Open-Tubular GlassCapillaries. Analytical Chemistry, 1981. 53(8): p. 1298-1302.

3. Huang, Y.F., C.C. Huang, C.C. Hu, and H.T. Chang, Capillary electrophoresis-based separation techniques for the analysis of proteins. Electrophoresis, 2006. 27(18): p. 3503-22.

4. Kraly, J., M.A. Fazal, R.M. Schoenherr, R. Bonn, M.M. Harwood, E. Turner, M. Jones, and N.J. Dovichi, Bioanalytical applications of capillary electrophoresis. Analytical Chemistry, 2006. 78(12): p. 4097-4110.

5. Liu, B.F., B. Xu, G. Zhang, W. Du, and Q.M. Luo, Micro-separation toward systems biology. Journal of Chromatography A, 2006. 1106(1-2): p. 19-28.

6. Dovichi, N.J. and J.Z. Zhang, How capillary electrophoresis sequenced the human genome. Angewandte Chemie-International Edition, 2000. 39(24): p. 4463-4468.

7. Haleem, J.I., The role of separation science in proteomics research. Electrophoresis, 2001. 22(17): p. 3629-3638.

8. Hancock, W.S., S.L. Wu, and P. Shieh, The challenges of developing a sound proteomics strategy. Proteomics, 2002. 2(4): p. 352-359.

9. Hjerten, S. and M.-D. Zhu, Micropreparative version of high-performance electrophoresis : The electrophoretic counterpart of narrow-bore high-performance liquid chromatography. Journal of Chromatography A, 1985. 327: p. 157-164.

10. Cohen, A.S., D.R. Najarian, A. Paulus, A. Guttman, J.A. Smith, and B.L. Karger, Rapid Separation and Purification of Oligonucleotides by High-Performance Capillary Gel Electrophoresis. Proceedings of the National Academy of Sciences of the United States of America, 1988. 85(24): p. 9660-9663.

11. Guttman,A., A.S. Cohen, D.N.Heiger, and B.L. Karger, Analytical and Micropreparative Ultrahigh Resolution of Oligonucleotides by Polyacrylamide-Gel High-Performance Capillary Electrophoresis. Analytical Chemistry, 1990. 62(2): p. 137-141.

12. Rose, D.J. and J.W. Jorgenson, Fraction collector for capillary zone electrophoresis. Journal of Chromatography A, 1988. 438: p. 23-34.

13. Huang, X.H. and R.N. Zare, Continuous sample collection in capillary zone electrophoresis by coupling the outlet of a capillary to a moving surface. Journal of Chromatography A, 1990. 516(1): p. 185-189.

14. Huang, X.H. and R.N. Zare, Use of an on-Column Frit in Capillary Zone Electrophoresis - Sample Collection. Analytical Chemistry, 1990. 62(5): p. 443-446. 
15. Cheng, Y.-F., M. Fuchs, D. Andrews, and W. Carson, Membrane fraction collection for capillary electrophoresis. Journal of Chromatography A, 1992. 608(1-2): p. 109-116.

16. Eriksson, K.O., A. Palm, and S. Hjerten, Preparative Capillary Electrophoresis Based on Adsorption of the Solutes (Proteins) onto a Moving Blotting Membrane as They Migrate out of the Capillary. Analytical Biochemistry, 1992. 201(2): p. 211-215.

17. Minarik, M., K. Klepárník, M. Gilár, F. Foret, A.W. Miller, Z. Sosic, and B.L. Karger, Design of a fraction collector for capillary array electrophoresis. Electrophoresis, 2002. 23(1): p. 35-42.

18. Muller, O., F. Foret, and B.L. Karger, Design of a High-Precision Fraction Collector for Capillary Electrophoresis. Analytical Chemistry, 1995. 67(17): p. 2974-2980.

19. Irie, T., T. Oshida, H. Hasegawa, Y. Matsuoka, T. Li, Y. Oya, T. Tanaka, G. Tsujimoto, and H. Kambara, Automated DNA fragment collection by capillary array gel electrophoresis in search of differentially expressed genes. Electrophoresis, 2000. 21(2): p. 367-374.

20. Minarik, M., F. Foret, and B.L. Karger, Fraction collection in micropreparative capillary zone electrophoresis and capillary isoelectric focusing. Electrophoresis, 2000. 21(1): p. 247-254.

21. Altria, K.D. and Y.K. Dave, Peak homogeneity determination and micro-preparative fraction collection by capillary electrophoresis for pharmaceutical analysis. Journal of Chromatography A, 1993. 633(1-2): p. 221-225.

22. Manz, A., N. Graber, and H.M. Widmer, Miniaturized Total Chemical-Analysis Systems - a Novel Concept for Chemical Sensing. Sensors and Actuators B-Chemical, 1990. 1(1-6): p. 244-248.

23. Manz, A., Y. Miyahara, J. Miura, Y. Watanabe, H. Miyagi, and K. Sato, Design of an Open-Tubular Column Liquid Chromatograph Using Silicon Chip Technology. Sensors and Actuators B-Chemical, 1990. 1(1-6): p. 249-255.

24. Manz, A., D.J. Harrison, E.M.J. Verpoorte, J.C. Fettinger, A. Paulus, H. Ludi, and H.M. Widmer, Planar Chips Technology for Miniaturization and Integration of Separation Techniques into Monitoring Systems - Capillary Electrophoresis on a Chip. Journal of Chromatography A, 1992. 593(1-2): p. 253-258.

25. Harrison, D.J., A. Manz, Z.H. Fan, H. Ludi, and H.M. Widmer, Capillary Electrophoresis and Sample Injection Systems Integrated on a Planar Glass Chip. Analytical Chemistry, 1992. 64(17): p. 1926-1932. 
26. Lee, S.H., S.I. Cho, C.S. Lee, B.G. Kim, and Y.K. Kim, Microfluidic chip for biochemical reaction and electrophoretic separation by quantitative volume control. Sensors and Actuators B-Chemical, 2005. 110(1): p. 164-173.

27. Belder, D., M. Ludwig, L.W. Wang, and M.T. Reetz, Enantioselective catalysis and analysis on a chip. Angewandte Chemie-International Edition, 2006. 45(15): p. 2463-2466.

28. Haswell, S.J., Chemical technology - All together now. Nature, 2006. 441(7094): p. 705-705.

29. Effenhauser, C.S., A. Manz, and H.M. Widmer, Manipulation of Sample Fractions on a Capillary Electrophoresis Chip. Analytical Chemistry, 1995. 67(13): p. 2284-2287.

30. Khandurina, J., T. Chovan, and A. Guttman, Micropreparative fraction collection in microfluidic devices. Analytical Chemistry, 2002. 74(7): p. 1737-1740.

31. Lin, R., D.T. Burke, and M.A. Burns, Selective extraction of size-fractioned DNA samples in microfabricated electrophoresis devices. Journal of Chromatography A, 2003. 1010(2): p. 255-268.

32. Lin, R., D.T. Burke, and M.A. Burns, Addressable Electric Fields for Size-Fractioned Sample Extraction in Microfluidic Devices. Analytical Chemistry, 2005. 77(14): p. 4338-4347.

33. Tulock, J.J., M.A. Shannon, P.W. Bohn, and J.V. Sweedler, Microfluidic separation and gateable fraction collection for mass-limited samples. Analytical Chemistry, 2004. 76(21): p. 6419-6425.

34. Strausbauch, M.A. and P.J. Wettstein, Fraction Collection with Micro-Preparative Capillary Electrophoresis, in Handbook of capillary electrophoresis, J.P. Landers, Editor. 1997, CRC Press. p. 841-864.

35. Woolley, A.T. and R.A. Mathies, Ultra-High-Speed DNA-Sequencing Using Capillary Electrophoresis Chips. Analytical Chemistry, 1995. 67(20): p. 3676-3680.

36. Miyashita, M., J.M. Presley, B.A. Buchholz, K.S. Lam, Y.M. Lee, J.S. Vogel, and B.D. Hammock, Attomole level protein sequencing by Edman degradation coupled with accelerator mass spectrometry. Proceedings of the National Academy of Sciences of the United States of America, 2001. 98(8): p. 4403-4408.

37. Chen, W., X. Yin, J. Mu, and Y. Yin, Subfemtomole level protein sequencing by Edman degradation carried out in a microfluidic chip. Chemical Communications, 2007(24): p. 2488-2490.

38. Dittrich, P.S. and A. Manz, Lab-on-a-chip: microfluidics in drug discovery. Nature reviews. Drug discovery, 2006. 5(3): p. 210-218. 
39. Bharadwaj, R., J.G. Santiago, and B. Mohammadi, Design and optimization of onchip capillary electrophoresis. Electrophoresis, 2002. 23(16): p. 2729-2744.

40. Wensink, H., H.V. Jansen, J.W. Berenschot, and M.C. Elwenspoek, Mask materials for powder blasting. Journal of Micromechanics and Microengineering, 2000. 10(2): p. $175-180$.

41. Vrouwe, E.X., R. Luttge, W. Olthuis, and A. van den Berg, Microchip analysis of lithium in blood using moving boundary electrophoresis and zone electrophoresis. Electrophoresis, 2005. 26(15): p. 3032-3042. 



\section{Chapter VI \\ Synchronized, Continuous-Flow \\ Zone Electrophoresis}

$\lceil$ electrophoretic separation of complex mixturesin microscale devices is proposed. Unlike in free-flow electrophoresis devices, no mechanical pumping is required - both fluid transport and separation are driven electrokinetically. This gives the method a great potential for on-a-chip integration in multistep analytical systems. The method enables to collect fractionated sample and tens-fold purification is possible. The model of the operation is presented and a detailed description of the optimal conditions for performing purification is given. The chip devices with $10 \mu \mathrm{m}$ deep separation chamber of $1.5 \mathrm{~mm} \times 4 \mathrm{~mm}$ in size were fabricated in glass. A standard microchip electrophoresis setup was used. Continuous separation of rhodamine B, rhodamine $6 \mathrm{G}$ and fluorescein was accomplished. Purification was demonstrated on a mixture containing rhodamine $B$ and fluorescein, and the recovery of both fractions was achieved. The results show the feasibility of the method.

This chapter is published in Analytical Chemistry, 2008, 80(16), 6228-6234. 


\section{Introduction}

The separation of multicomponent samples plays an important role in (bio) analytical sciences. Many traditional separation methods have been downscaled to microfluidic format and numerous applications of such miniaturized systems have been shown. ${ }^{1,2}$ Continuous separation techniques offer clear advantages over batch-type systems ${ }^{3}$ and various microscale, continuous separation devices have been demonstrated, ${ }^{4-10}$ including both scaled-down counterparts of traditional instruments and systems based on newly discovered phenomena. Among them, free-flow zone electrophoresis ${ }^{11}$ (FFZE) and free-flow isoelectric focusing ${ }^{12}$ (FFIF) have recently gained attention and several groups reported their progress with these methods. ${ }^{13-17}$ Yet, despite their development, the free-flow electrophoretic techniques share a weakness - both mechanical pumping and high-voltage supply are required for their operation. Usually additional time is required to perform preparation steps specific to a hydraulic setup (e.g. cleaning of external fluidic system, making pressure-resistant connections). Moreover the presence of the pressure driven flow within the device may lead to difficulties during the on-a-chip integration with follow-up electrokinetic postprocessing (e.g. orthogonal separation methods).

In this paper we demonstrate a new method of continuous sample separation by zone electrophoresis. Unlike in the free-flow electrophoresis, no mechanical pumping is required; the device relies on electrokinetic flow control only, greatly reducing its complexity. The separation is performed in a microfluidic rectangular chamber, having three inlets on one side and three outlets on the opposite side. A constant-velocity flow is forced in the chamber by applying high voltages (up to $1 \mathrm{kV}$ ) to the inlets and the outlets. A sample is injected into the chamber through the middle inlet channel, and is electrokinetically focused ${ }^{18}$ to form a narrow stream, sandwiched between two sheath streams containing a buffer solution only. The lateral position of the sample stream at the chamber entrance is varied over time in a predefined way, similarly to the continuous electrophoresis in rectangular channels method. ${ }^{19,} 20$ This, combined with an axial electric field, produces a wavelike sample stream pattern in the chamber. Due to the differences in apparent mobilities of the sample components, a separation of the sample in axial direction occurs. This is observed as travelling waves with different periods, each wave belonging to one component. By employing synchronized switching of the voltages applied to the outlet channels, as explained in the experimental section, one of the 
separated components can be collected into the middle outlet channel. This new method, called by us a synchronized, continuous-flow zone electrophoresis has a number of potential applications as a pre-fractionation or purification method in multidimensional separation systems. It also can be applied in integrated chemical microreactors ${ }^{21}$ for monitoring reaction rate or removal of unwanted products. Its great advantage is a lack of pressure driven flow which simplifies interfacing with other purely electrokinetic systems.

\section{Experimental section}

\section{Theory of operation}

To explain the principle of the method, we refer to Figure 1. The basic device assembly consists of a microfluidic laminar-flow chamber with three inlets and three outlets. The flow within the device is induced electrokinetically and controlled by adjusting high voltages applied to the inlets and the outlets in an appropriate manner. The middle inlet is used for introducing a sample into the chamber, whereas the two outer inlets provide sheath streams for manipulating the position and the width of the sample stream by electrokinetic steering. ${ }^{22}$ The presence of the transverse electric field in the vicinity of the entrance and the exit of the chamber is neglected. The electric field throughout the separation chamber $E_{S}$ is assumed to be uniform; also the flow within the chamber is considered to be a fully developed plug-flow. During the operation the starting position of the sample stream $y_{S}$ is varied periodically according to:

$$
y_{s}(t)=A \sin (\omega t)
$$

where $A$ - the amplitude, $\omega$ - the angular frequency of the oscillation. This, combined with the flow in the axial direction $(x)$, driven by the electric field $E_{S}$, results in a wavelike sample stream path, which takes the theoretical form:

$$
y(x, t)=-A \sin \left(\omega\left(\frac{x}{\mu_{S} E_{S}}-t\right)\right)
$$

Here $\mu_{s}$ denotes the apparent mobility of the sample. The real shape of this curve near the chamber exit is determined by the flow-rates of the outlet streams. If, in the case of the sample stream pattern shown in Figure 1a, the sheath outlet fluxes are equal and much greater than the middle outlet flux, the sample stream will be bent towards the upper outlet. However, it is possible to adjust the steering voltages in 
such a way, that the sample stream will exit through the middle outlet. To achieve this, a switching pattern similar to at the inlet of the chamber must be employed at the outlet. In the case presented in Figure $1 \mathrm{a}$, increasing the flux $\phi_{6}$, while lowering the flux $\phi_{4}$ and keeping the middle outlet flux at the level of the sample inlet flux should result in guiding the sample stream into the middle outlet.
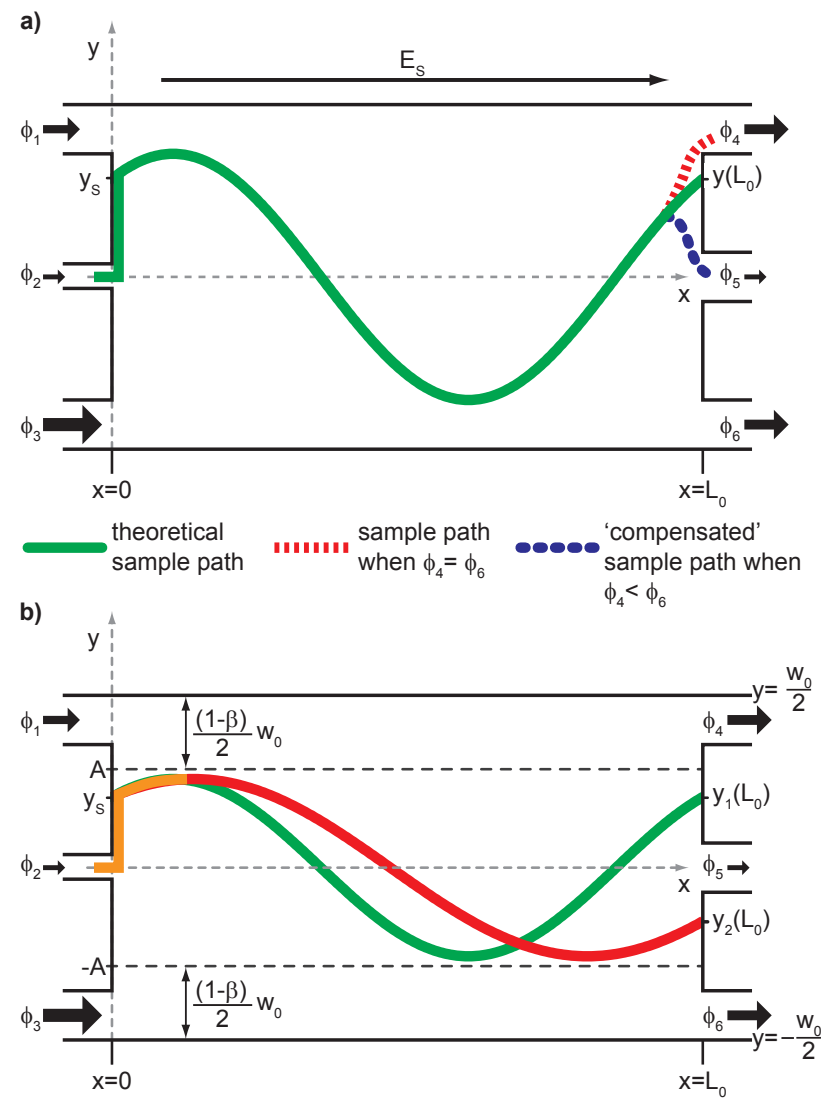

Figure 1. Principle of synchronized, continuous-flow zone electrophoresis; a) altering outlet fluxes for continuous sample collection; b) two components case.

If a multicomponent mixture is used as a sample, and the components differ in apparent mobilities, additional sample waves, with shapes adhering to (2) will be present in the chamber (Figure 1b). A steering scheme, where the outlet fluxes are continuously altered to guide one of the exiting component streams into the middle outlet channel, results in the collection of its purified fraction. We refer to this effect as to synchronized, continuous-flow zone electrophoresis (SCFZE). 


\section{Synchronized steering}

For controlling the separation process, the running electrolyte properties (conductivity $\sigma_{0}$ and mobility $\mu_{0}$ ), as well as exact dimensions of the chamber, inlet and outlet channels must be known. Prior to operation the following values should be also provided: the electric field strength $-E_{S}$, the sample stream width given as a fraction of the chamber width $-\alpha$, the sample confinement coefficient $-\beta$ (i.e. a fraction of the chamber width, where a sample wave is present - see Figure $1 b$ ), the angular frequency $\omega$ at which the sample stream starting position varies according to (1), and the apparent mobility of the component to be collected $-\mu_{s}$, which is the sum of the electrophoretic and electroosmotic mobilities. The total flux through the chamber is then given as:

$$
\phi_{0}=\mu_{0} E_{S} S_{0}
$$

where $S_{0}$ is the cross-sectional area of the chamber. Consequently, the fluxes through the inlets and outlets, as functions of time, can be derived:

$$
\begin{aligned}
& \phi_{2}(t)=\phi_{5}(t)=\alpha \phi_{0} \\
& \phi_{1}(t)=\phi_{\min }+\frac{A-y_{i}(0, t)}{2 A}(\beta-\alpha) \phi_{0} \\
& \phi_{3}(t)=\phi_{\min }+\frac{A+y_{i}(0, t)}{2 A}(\beta-\alpha) \phi_{0} \\
& \phi_{4}(t)=\phi_{\min }+\frac{A-y_{i}\left(L_{0}, t\right)}{2 A}(\beta-\alpha) \phi_{0} \\
& \phi_{6}(t)=\phi_{\min }+\frac{A+y_{i}\left(L_{0}, t\right)}{2 A}(\beta-\alpha) \phi_{0}
\end{aligned}
$$

The function $y_{i}(x, t)$ defined in (2) is evaluated for the mobility of a collected component, and $L_{0}$ denotes the length of the chamber. The amplitude $A$ of the wave equals:

$$
A=\frac{\beta-\alpha}{2} w_{0}
$$

where $w_{0}$ is the width of the chamber. The minimum sheath stream flux $\phi_{\min }$ is derived from the confinement coefficient:

$$
\phi_{\min }=\frac{1-\beta}{2} \phi_{0}
$$


The electric currents flowing through the inlet and outlet channels can be obtained from the fluxes by using the dependency:

$$
i_{i}(t)=\phi_{i}(t) \frac{\sigma_{0}}{\mu_{0}}
$$

Then, the relations between the steering voltages that need to be applied to the channels are derived by using Kirchhoff's laws ${ }^{23,24}$ and resistance values of the channels and the chamber evaluated from then known device dimensions. Since the system of equations produced by such analysis in unsolvable, the lowest applied potential (one of the side outlet channels) is assumed to equal zero, which allows evaluating the remaining potentials.

\section{Microchip fabrication}

The chip was manufactured in borosilicate glass, utilizing standard microfabrication techniques. ${ }^{25}$ Briefly, two glass plates were used; the top plate contains the fluidic channels as well as reservoir openings. The channels were created by etching in hydrofluoric acid through a patterned $\mathrm{Cr} / \mathrm{Au}$ mask. The reservoirs openings were fabricated by powderblasting with $\mathrm{Al}_{2} \mathrm{O}_{3}$ particles through a patterned polymer photoresist foil. Next, the resting masking material was removed and the wafers were thermally bonded. Finally the bonded wafer stack was diced into separate chip devices.

\section{Chemicals}

All chemicals were purchased from Sigma-Aldrich-Fluka. A 20 mM MES/Histidine at pH 6.35 was used as a buffer. The two-component sample contained $500 \mu \mathrm{M}$ rhodamine $\mathrm{B}, 750 \mu \mathrm{M}$ fluorescein in a buffer solution. The three component sample consisted of $100 \mu \mathrm{M}$ rhodamine $\mathrm{B}, 50 \mu \mathrm{M}$ fluorescein and $100 \mu \mathrm{M}$ rhodamine $6 \mathrm{G}$ in a buffer solution. All fluids contained 3\% (v/v) ethanol to improve solubility of sample components and $0.05 \%(\mathrm{w} / \mathrm{v}$ ) Tween 20 to overcome difficulties with filling the separation chamber. Solutions were filtered through a $0.22 \mu \mathrm{m}$ membrane filter and degassed for 5 minutes in a vacuum chamber.

\section{Apparatus and procedures}

The chips were stored in demineralised water. Prior to experiments, they were placed in a custom-made holder and flushed for 5 minutes with the buffer solution. Then, the buffer in the reservoirs was exchanged and a sample mixture was introduced into the middle inlet reservoir. Following this, the experiments 
were started immediately. Two computer-controlled, high-voltage power supplies (IBIS B.V., Hengelo, the Netherlands) were used to steer the device. They were controlled by a native Windows ${ }^{\mathrm{TM}}$ application, written in-house, with $40 \mathrm{~Hz}$ frequency (i.e. the voltages were updated in $25 \mathrm{~ms}$ intervals). The images were captured with a digital colour camera ColorView II (Olympus) mounted to a fluorescence microscope (Olympus IX51) equipped with a mercury lamp and a fluorescent filter sets (XF57, Omega Optical, USA and 11012v2, Chroma Technology, USA). The exposure time for the images was $100 \mathrm{~ms}$ unless otherwise stated. Numerical computations were performed in Matlab 7 software package using built-in numerical integration functions and a self-written trapezoidal rule integration function.

\section{Results and discussion}

\section{Chip devices}

A fabricated chip is shown in Figure 2. The chips are $20 \mathrm{~mm} \times 15 \mathrm{~mm}$ in size. The etched channels depth is $10 \mu \mathrm{m}$. The side inlets and outlets are $500 \mu \mathrm{m}$ wide and $4300 \mu \mathrm{m}$ long. Both the sample inlet channel and the sample outlet channel consist of two parts with different dimensions. For the inlet they are: $1200 \mu \mathrm{m}$ long, $200 \mu \mathrm{m}$ wide and $900 \mu \mathrm{m}$ long, $100 \mu \mathrm{m}$ wide respectively; and for the outlet: $750 \mu \mathrm{m}$ long, $100 \mu \mathrm{m}$ wide and $1350 \mu \mathrm{m}$ long, $500 \mu \mathrm{m}$ wide. The separation chamber dimensions are $4000 \mu \mathrm{m} \times 1500 \mu \mathrm{m}$.

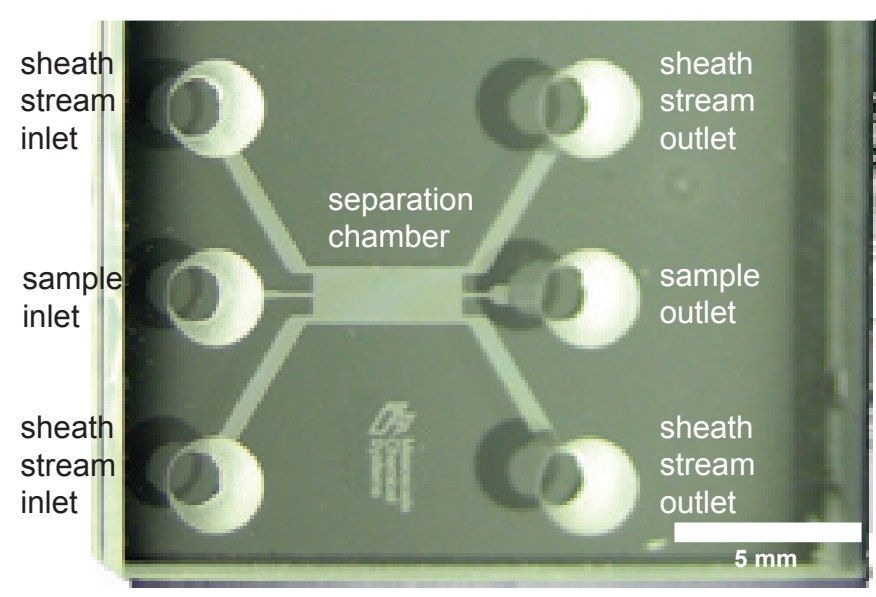

Figure 2. A photograph of the fabricated chip devices. 


\section{Separation}

Table 1 summarizes the parameters values used in the experiments.

Table 1. List of parameters used during the separation and purification experiments

\begin{tabular}{|c|c|c|c|c|c|}
\hline & $\begin{array}{l}E_{S} \\
V^{\prime} \cdot \mathbf{c m}^{-1}\end{array}$ & $\begin{array}{l}\mu_{S_{2}} \cdot(V \cdot s)^{-1} \\
m^{2}\end{array}$ & $\begin{array}{l}\omega \\
\mathrm{rad} \cdot \mathrm{s}^{-1}\end{array}$ & $\alpha$ & $\beta$ \\
\hline $\begin{array}{l}\text { Separation, } \\
2 \text { components }\end{array}$ & 300 & $1.97 \cdot 10^{-8}$ & $\pi$ & 0.01 & 0.9 \\
\hline $\begin{array}{l}\text { Separation, } \\
3 \text { components }\end{array}$ & 350 & $3.55 \cdot 10^{-8}$ & $0.5 \cdot \pi$ & 0.01 & 0.8 \\
\hline $\begin{array}{l}\text { No } \\
\text { synchronization }\end{array}$ & 400 & $2.35 \cdot 10^{-8}$ & $0.5 \cdot \pi$ & 0.005 & 0.4 \\
\hline $\begin{array}{l}\text { Rhodamine B } \\
\text { synchronization }\end{array}$ & 400 & $1.97 \cdot 10^{-8}$ & $0.5 \cdot \pi$ & 0.005 & 0.4 \\
\hline $\begin{array}{l}\text { Fluorescein } \\
\text { Synchronization }\end{array}$ & 400 & $1.02 \cdot 10^{-8}$ & $0.5 \cdot \pi$ & 0.01 & 0.4 \\
\hline
\end{tabular}

The separation tests were performed on both sample mixtures, one containing two components and another three components. Figure 3 shows a sequence of images taken near the chamber entrance during the separation of rhodamine B and fluorescein mixture. The separation of the sample occurs as predicted by the theory. However, the amplitude of the fluorescein wave is lower than the rhodamine wave amplitude. This phenomenon is caused by the difference in the mobilities of the components (rhodamine B is neutral, while fluorescein is negatively charged, thus the net mobility of rhodamine B is greater than of fluorescein ${ }^{26}$ ). In fact, to modulate the sample wave properly, the following condition must be valid:

$$
\frac{d y_{S}(t)}{d t} \leq \mu_{s} E_{T}
$$

where $E_{T}$ is the transient, transverse electric field, present in a small region near the chamber entrance, ${ }^{22}$ which causes the sample stream starting position to move up and down. This effect is not considered in the presented theory of SCFZE, where uniformity of the electric field in the chamber is assumed. Its influence can be avoided by lowering the modulation frequency or the amplitude to obey the condition in equation (8). The visible, relatively large width of the components streams is partially caused by long exposure times that were used due to the equipment limitations. 

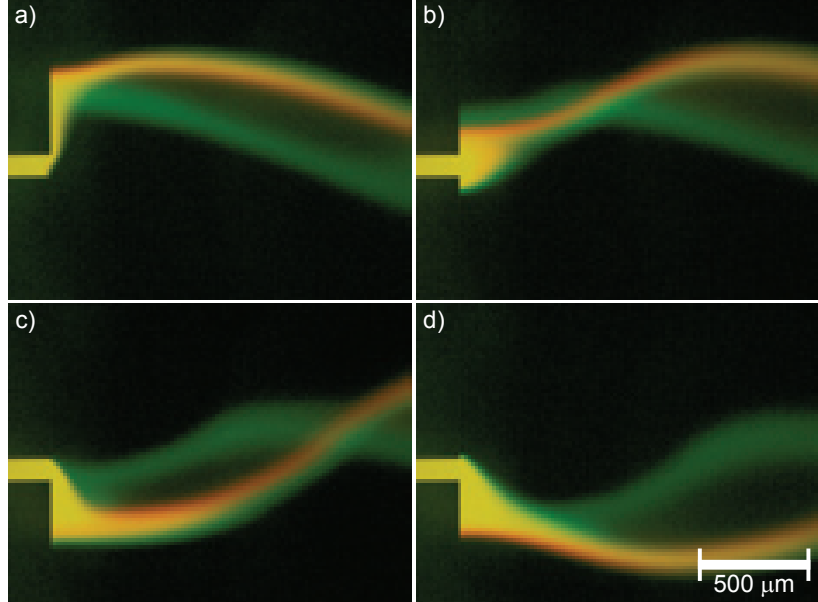

Figure 3. Separation of rhodamine B (red/orange) and fluorescein (green) mixture. The amplitude of fluorescein wave is visibly smaller than that of rhodamine due to its lower mobility.

To test the separation efficiency for species with smaller difference in mobilities, a three component mixture was used containing fluorescein, rhodamine $\mathrm{B}$ and rhodamine $6 \mathrm{G}$.
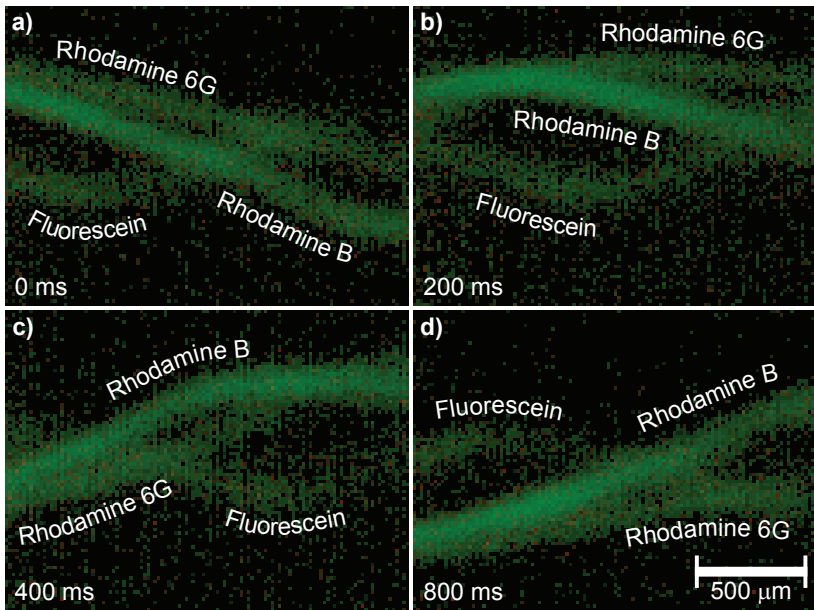

Figure 4. Separated component streams of rhodamine B, rhodamine $6 \mathrm{G}$ and fluorescein. The separation between the rhodamine dyes is significantly worse near the waves maxima. (Different colour scheme of this image is caused by the use of a different filter set - necessary, due to low dyes concentrations)

Figure 4 shows pictures of separated sample streams taken approximately at $1 / 3$ of the chamber length. The differences in the net mobilities of the components $\left(\left|\mu_{\text {rho6G }}-\mu_{\text {rhoB }}\right|<\left|\mu_{\text {rhoB }}-\mu_{\text {fluo }}\right|\right)$ result in significant differences in spatial separation 
between the streams. Moreover the spacing between the two rhodamine dyes is not evident near the maxima of the waves. This effect is caused both by the diffusion and the shape of the waves, and can be minimized by changing the amplitude modulation function (1).

\section{Synchronized collection and purification}

The demonstration of SCFZE was performed on a sample containing fluorescein and rhodamine $\mathrm{B}$. The values of the mobilities of the components, needed for accurate synchronization were found experimentally by starting the process with synchronization to the sample mobility $\mu_{s}=4.0 \cdot 10^{-8} \mathrm{~m}^{2} \cdot(\mathrm{Vs})^{-1}$ and lowering its value in $0.05 \cdot 10^{-8} \mathrm{~m}^{2} \cdot(\mathrm{Vs})^{-1}$ steps. Figure $5 \mathrm{a}-\mathrm{d}$ shows typical paths of the components streams in the chamber exit region, when neither component is synchronized. Both component streams are being swept over the whole width of the chamber. As a result, the components enter all three outlets periodically and no collection of a purified component occurs. Corresponding images of the sample collection channel can be seen in Figure 5e,f, where similar amount of both components enters the channel and is collected. This is observed as alternating strips of fractions separated by pure buffer volumes. The broadening of the streams in the vicinity of the outlets, most pronounced in Figure $5 \mathrm{a}$, is caused by the electric field distribution present in the electrokinetic guiding scheme ${ }^{22}$ and the diffusion of the sample.

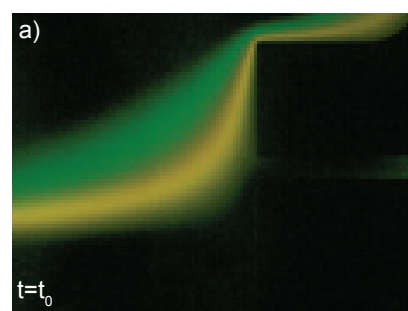

d)

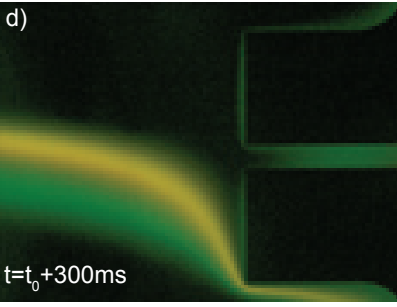

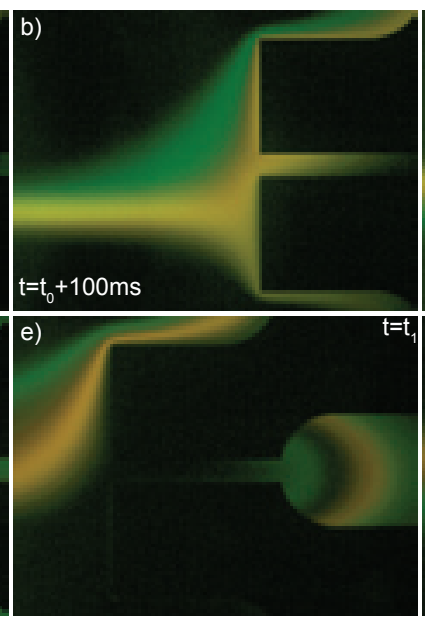

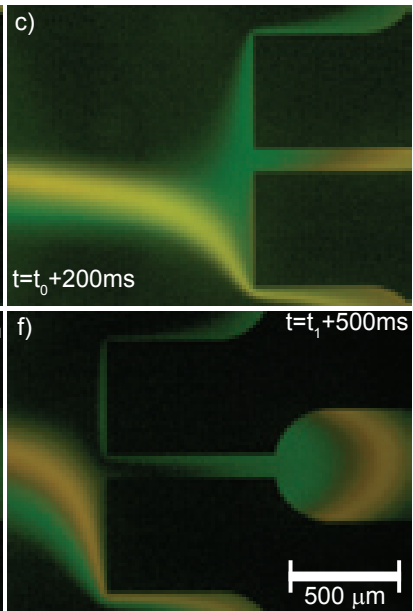

Figure 5. Unsynchronized fractionation of two-component sample; a-d - typical component streams; $\mathrm{e}, \mathrm{f}-$ collection of both components into the sample outlet channel occurs.

The values of the apparent mobilities for which the synchronization was observed were $1.97 \cdot 10^{-8} \mathrm{~m}^{2} \cdot(\mathrm{Vs})^{-1}$ for rhodamine B and $1.02 \cdot 10^{-8} \mathrm{~m}^{2} \cdot(\mathrm{Vs})^{-1}$ for fluorescein. The 
remaining parameters can be found in Table 1 . The image sequence taken during the synchronized collection of rhodamine B is shown in Figure $6 \mathrm{a}-\mathrm{d}$. The most distinct difference, as compared to the unsynchronized fractionation case shown in Figure 5, is that the rhodamine fraction stream, despite its original position, now always enters the middle collection channel. This is accomplished by adjusting the magnitudes of the outlet fluxes according to equation (4). The result of this action is observed, near the end of the chamber, as a deflection of the rhodamine stream towards the middle outlet. The remaining fraction (fluorescein) is still being swept over the whole chamber width as in the case of unsynchronized fractionation.

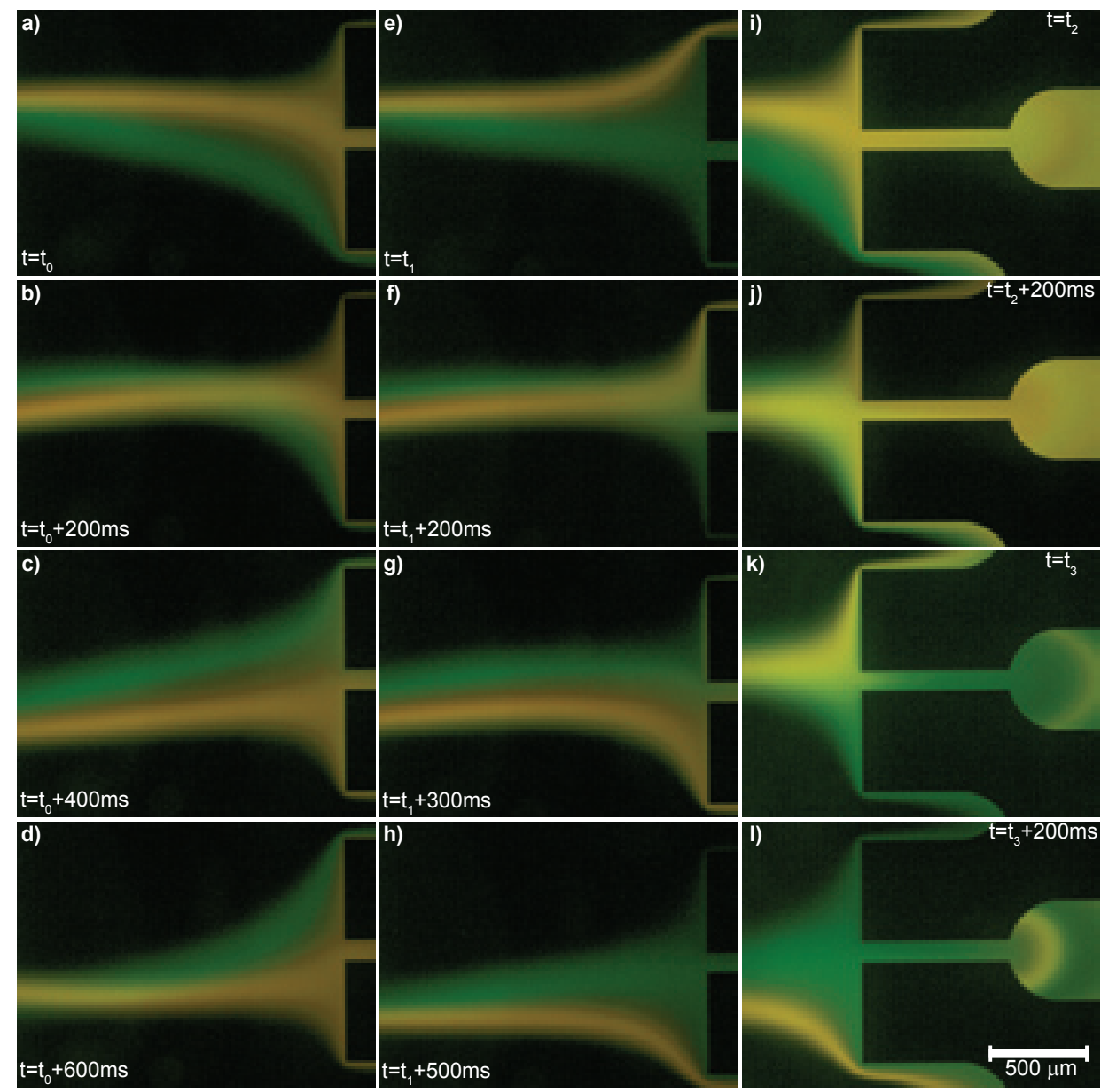

Figure 6. Sequence of images taken during the synchronized fractionation of a rhodamine $B$, fluorescein mixture: $a-d-$ collection of rhodamine $B$ and $e-h-$ collection of fluorescein. Corresponding images of outlet channel for $i, j$ - rhodamine B collection and $\mathrm{k}, \mathrm{I}-$ fluorescein collection. 
Likewise, the collection of purified fluorescein fraction is shown in Figure 6e-h. Again a characteristic deflection can be seen.

The corresponding images of the sample outlet channel are presented in Figure 6i,j and Figure 6k,l for the synchronized collection of rhodamine B and fluorescein, respectively. The collected fractions are visually pure with an exception for small contamination rings (better visible, due to the used optical filter set characteristics, in the case of fluorescein collection).

The broadening of the collected sample streams, seen in Figure 6, is caused by the electric field distribution, similarly as observed for Figure 5. Despite some sample loss caused by this effect, it is negligible for purification or fractionation purposes.

\section{Contamination}

The contamination of a collected component by unsynchronized fractions, as seen in Figure 6, is unavoidable. In principle, the contamination occurs when the lateral positions at the end of the chamber of both, the synchronized and the unsynchronized sample streams are equal:

$$
y_{s}\left(L_{0}, t\right)=y_{u s}\left(L_{0}, t\right)
$$

where $y\left(L_{0^{\prime}} t\right)$ is defined by equation (2). When it happens, the unsynchronized sample stream is forced to enter the middle outlet together with the collected component. Equation (9) has two solutions. One of them is given by:

$$
\frac{1}{\mu_{s}}-\frac{1}{\mu_{u s}}=\frac{2 k \pi E_{s}}{\omega L_{0}} \quad k=0, \pm 1, \pm 2, \ldots
$$

This solution is independent of time. It means that, when the mobility of the collected component $\mu_{s}$ and the mobility of the unsynchronized fraction $\mu_{u s}$ satisfy the condition given in (10), the streams overlap at any instant of time at the end of the chamber, thus the contamination occurs continuously. Equation (10) can be used to generate, by varying $k$, a set of mobilities for a given sample mobility $\mu_{s}$ and parameters $E_{S}, \omega, L_{0}$. The fractionation of a mixture, containing components with mobilities belonging to such set, is impossible under the conditions described by the parameters used to generate it. Yet, they can be still fractionated by changing either the separation field $E_{S}$ or the angular frequency of modulation $\omega$, in such a way, that the condition defined in (10) is not valid. 
Another solution can be obtained by solving (9) for time $t$. It shows that for the samples and the fractionation conditions that do not satisfy (10) the contamination occurs periodically with the frequency:

$$
f_{\text {cont }}=\frac{\omega}{\pi}
$$

In the experiments of synchronized fractionation of the rhodamine B / fluorescein mixture, described in this report, $f_{\text {cont }}=0.5 \mathrm{~Hz}$. The interpretation of equation (11) can be misleading. It shows that lowering the modulation frequency results in less frequent occurrence of the unsynchronized stream entering the sample outlet. However, the initial width of the streams and the lateral diffusion are not considered in equation (9), and thus in its solutions - the sample streams are assumed to be infinitesimally narrow. As a consequence, $f_{\text {cont }}$ does not provide any quantitative information about the contamination level. To assess the amount of contamination we performed numerical computation.

On the assumption, that the electric field in the chamber $E_{s}$ is uniform, the concentration function of a sample with the apparent mobility $\mu_{i}$ and the stream path $y_{i}(x, t)$ defined in equation (2) is given by ${ }^{27}$ :

$$
C_{i}(x, y, t)=\frac{C_{0, i}}{2}\left[\operatorname{erf}\left(\frac{\frac{\alpha w_{0}}{2}-y+y_{i}(x, t)}{2 \sqrt{D_{i} \frac{x}{\mu_{i} E_{S}}}}\right)+\operatorname{erf}\left(\frac{\frac{\alpha w_{0}}{2}+y-y_{i}(x, t)}{2 \sqrt{D_{i} \frac{x}{\mu_{i} E_{S}}}}\right)\right]
$$

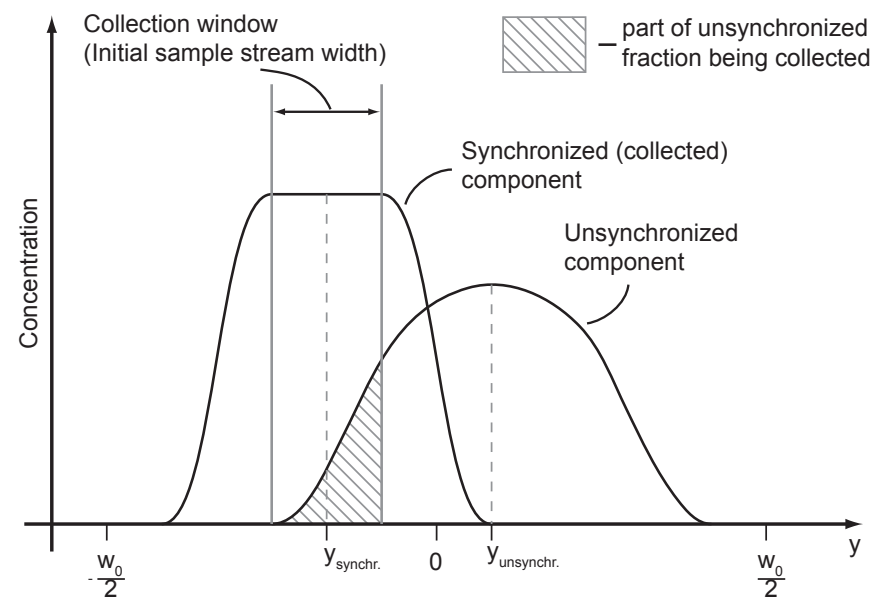

Figure 7. Schematic representation of concentration profiles of two diffusing sample streams at the end of the separation chamber. 
Figure 7 shows a schematic plot of lateral concentration profiles of two components at the end of the separation chamber $\left(x=L_{0}\right)$. The middle point $y_{\text {unsynchr. }}$ of the unsynchronized component lies outside the collection window. However, due to the diffusion, a part of it is collected together with the synchronized fraction, causing contamination.

For a mixture containing n components, the total collected amount of material of the $i$-th component $m_{i}$, during the synchronized collection of the $j$-th component, in the time interval $T$, can be calculated by solving the double integral:

$$
m_{i}=\mu_{i} E_{S} \cdot \int_{T} \int_{y_{j}-0.5 \alpha_{0}}^{y_{j}+0.5 \alpha_{w 0}} C_{i}\left(L_{0}, y, t\right) d y d t
$$

where $y_{j}$, present in the integration limits, is the position function (2) of the synchronized component evaluated for $x=L_{0}$. The numerical computations of the integral (13) were performed for a two component mixture. The parameters used for the calculations were: the mobilities of the components $\mu_{1}=1.97 \cdot 10^{-8} \mathrm{~m}^{2} \cdot(\mathrm{Vs})^{-1}$, $\mu_{2}=1.02 \cdot 10^{-8} \mathrm{~m}^{2} \cdot(\mathrm{Vs})^{-1}$, the diffusivities of the components $D_{1}=D_{2}=1 \cdot 10^{-10} \mathrm{~m}^{2} \cdot \mathrm{s}^{-1}$, the separation field $E_{S}=400 \mathrm{~V} \cdot \mathrm{cm}^{-1}$, and the stream width coefficient $\alpha=0.01$. The physical dimensions of the chip device described in this article were used. Figure 8 shows the results of the calculations. The fractionation selectivity versus the modulation frequency is plotted, for the collection of the faster (Figure 8a) and the slower (Figure 8b) components. The fractionation selectivity was calculated as:

$$
S=\frac{M_{u s} \cdot m_{s}}{m_{u s} \cdot M_{s}}
$$

where $M_{s}, M_{u s}$ are the total masses entering the separation chamber of the synchronized and unsynchronized components respectively, and $m_{s}, m_{u s}$ are the collected masses of the synchronized and unsynchronized components. When the selectivity drops below 1, a bigger part of the unsynchronized component flowing through the chamber is collected than of the synchronized component.

As can be seen in Figure 8, the selectivity exhibits periodic variations, and reaches the maximum values for:

$$
\omega=\frac{(2 k+1) \pi \mu_{s} \mu_{u s} E_{s}}{L_{0}\left|\mu_{s}-\mu_{u s}\right|} \quad k=0,1,2, \ldots
$$



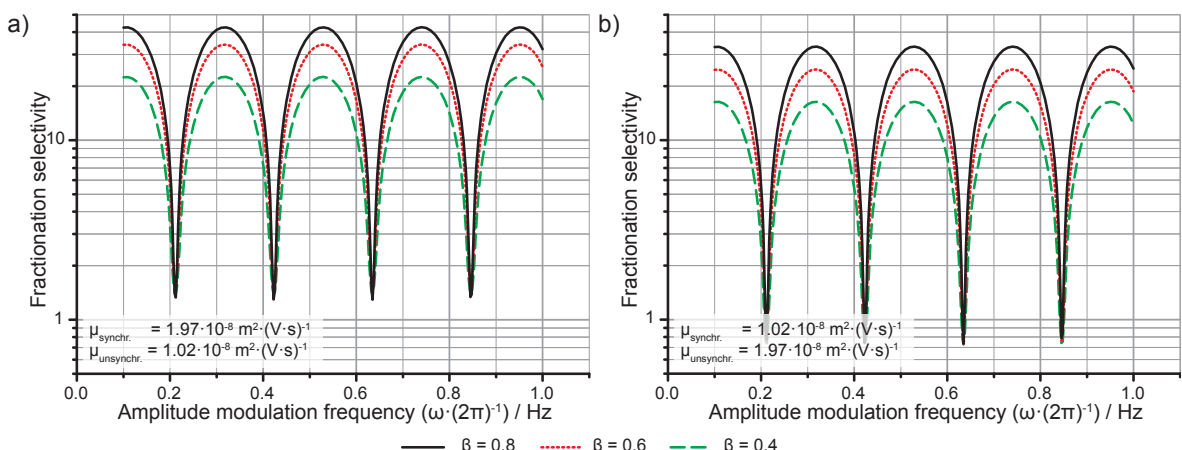

$-\beta=0.8$

$$
\beta=0.6--\beta=0.4
$$

Figure 8. Fractionation selectivity for the synchronized fractionation of a two-component mixture. Selectivity for collection of the component with higher (a) and lower (b) mobility. The selectivity exhibits periodic variations, dependent on the amplitude modulation frequency.

The selectivity values depend not only on the amplitude modulation frequency $\omega$ and the sample confinement coefficient $\beta$, but also on the sample chosen for collection. This effect is caused by the difference in the mobilities of components. The mobility determines not only the flux of a component, but also the residence time in the chamber (i.e. the time needed for the sample to travel the distance of the chamber length). Thus, the diffusional dispersion at the end of the chamber of the sample stream of the less mobile component is greater than that of the more mobile component. As a consequence of these phenomena, less material of the slower component is collected per time unit than of the faster component, even if their streams overlap continuously at the collection point (selectivity minima in Figure 8).

Table 2 summarizes the maximum and minimum values of purification ratios derived for the computed fractionation selectivities. The purification ratio is defined as:

$$
\text { Pur }=\frac{C_{s}}{C_{u s}} \frac{C_{u s}^{*}}{C_{s}^{*}}=\frac{\mu_{s}}{\mu_{u s}} S
$$

where $C$ is the concentration of the collected component and $C^{*}$ is its initial concentration. Ten-fold purification can be easily achieved for the tested mixture, and much higher rates are possible by adjusting fractionation parameters. 
Table 2. Maximum and minimum purification ratios for the synchronized fractionation of the two-component mixture, with component mobilities $\mu_{1}=1.97 \cdot 10^{-8} \mathrm{~m}^{2} \cdot(\mathrm{Vs})^{-1}$ and $\mu_{2}=1.02 \cdot 10^{-8} \mathrm{~m}^{2} \cdot(\mathrm{Vs})^{-1}$.

\begin{tabular}{|c|c|c|c|c|}
\hline & & Pur $_{\text {ma }}$ & & Pur $_{\min }$ \\
\hline & $\beta=0.4$ & $\beta=0.6$ & $\beta=0.8$ & $\beta$-independent \\
\hline $\begin{array}{l}\mu_{s}=1.97 \cdot 10^{-8} \\
\mathrm{~m}^{2} \cdot(\mathrm{Vs})^{-1}\end{array}$ & 43.51 & 65.92 & 88.32 & 2.66 \\
\hline $\begin{array}{l}\mu_{\text {S }}=1.02 \cdot 10^{-8} \\
\mathrm{~m}^{2} \cdot(\mathrm{Vs})^{-1}\end{array}$ & 8.48 & 12.84 & 17.20 & 0.38 \\
\hline
\end{tabular}

\section{Conclusions}

Microfluidic, synchronized, continuous-flow zone electrophoresis was successfully demonstrated for the first time. The theoretical model of the method was presented and applied in the experimental setup. The results positively validate the model. Continuous separation and continuous fractionation with collection of the purified components were achieved in the electrokinetic-only microchip device. The theoretical explanation of the influence of the fractionation parameter on the efficiency of the collection process was given, together with the derivation of the optimal values. By choosing appropriate operating parameters it is possible to perform tens-fold purification by SCFZE. Synchronized, continuous-flow zone electrophoresis can be relatively easily performed by extending standard microchip CE setups. Further improvement of the method is possible, both theoretical and practical by e.g. optimizing the device geometry, changing the amplitude modulation function and extending the theoretical description to include the effects not considered in the current model. SCFZE has a potential broad application area as a purification or pre-fractionation tool in integrated separation and analytical systems.

\section{Symbols}

$E_{S}-$ electric field throughout the separation chamber

$\mathrm{y}_{\mathrm{s}}-$ starting position of a sample stream

$t$ - time

$A$ - amplitude of a sample stream wave

$\omega-$ angular frequency of oscillation

$y(x, t)$ - shape function of a sample stream

$\mu_{S}-$ apparent mobility of a synchronized sample

$\mu_{u s}$ - apparent mobility of an unsynchronized sample 
$\phi_{1,3}$ - inlet sheath fluxes

$\phi_{4,6}$ - outlet sheath fluxes

$\phi_{2}-$ inlet sample flux

$\phi_{5}-$ outlet sample flux

$\sigma_{0^{-}}$conductivity of a running electrolyte

$\mu_{0}-$ mobility of a running electrolyte

$\alpha-\quad$ ratio of sample stream width and chamber width

$\beta$ - sample confinement coefficient (i.e. a fraction of the chamber width, where a sample wave is present

$\phi_{0}-$ total flux through a chamber

$L_{0}$ - length of a separation chamber

$w_{0}$ - width of a separation chamber

$\phi_{\min }-$ minimum sheath stream flux

$E_{T}$ - transient, transverse electric field, present in a small region near the chamber entrance

$f_{\text {cont }}$ - frequency at which contamination occurs

$C_{0 i}$ - initial sample concentration

$D_{i}$ - diffusion coefficient

$S$ - fractionation selectivity

$M_{s}$ - total mass of a synchronized component entering a separation chamber

$M_{u s}$ - total mass of an unsynchronized component entering a separation chamber

$m_{s}$ - collected mass of a synchronized component

$m_{u s}$ - collected mass of an unsynchronized component

C - concentration of a collected component

$C^{*}$ - initial concentration of a collected component

Pur-purification ratio

\section{References}

1. Vilkner, T., D. Janasek, and A. Manz, Micro total analysis systems. Recent developments. Analytical Chemistry, 2004. 76(12): p. 3373-3385.

2. Dittrich, P.S., K. Tachikawa, and A. Manz, Micro total analysis systems. Latest advancements and trends. Analytical Chemistry, 2006. 78(12): p. 3887-3907. 
3. Pamme, N., Continuous flow separations in microfluidic devices. Lab on a Chip, 2007. 7(12): p. 1644-1659.

4. Han, J. and H.G. Craighead, Separation of long DNA molecules in a microfabricated entropic trap array. Science, 2000. 288(5468): p. 1026-1029.

5. Pamme, N. and A. Manz, On-chip free-flow magnetophoresis: Continuous flow separation of magnetic particles and agglomerates. Analytical Chemistry, 2004. 76(24): p. 7250-7256.

6. Janasek, D., M. Schilling, J. Franzke, and A. Manz, Isotachophoresis in free-flow using a miniaturized device. Analytical Chemistry, 2006. 78(11): p. 3815-3819.

7. Song, Y.A., S. Hsu, A.L. Stevens, and J.Y. Han, Continuous-flow pl-based sorting of proteins and peptides in a microfluidic chip using diffusion potential. Analytical Chemistry, 2006. 78(11): p. 3528-3536.

8. Choi, S. and J.K. Park, Continuous hydrophoretic separation and sizing of microparticles using slanted obstacles in a microchannel. Lab on a Chip, 2007. 7(7): p. 890-897.

9. Fu, J.P., R.B. Schoch, A.L. Stevens, S.R. Tannenbaum, and J.Y. Han, A patterned anisotropic nanofluidic sieving structure for continuous-flow separation of DNA and proteins. Nature Nanotechnology, 2007. 2(2): p. 121-128.

10. Petersson, F., L. Aberg, A.M. Sward-Nilsson, and T. Laurell, Free flow acoustophoresis: Microfluidic-based mode of particle and cell separation. Analytical Chemistry, 2007. 79(14): p. 5117-5123.

11. Raymond, D.E., A. Manz, and H.M. Widmer, Continuous Sample Pretreatment Using a Free-Flow Electrophoresis Device Integrated onto a Silicon Chip. Analytical Chemistry, 1994. 66(18): p. 2858-2865.

12. Xu, Y., C.X. Zhang, D. Janasek, and A. Manz, Sub-second isoelectric focusing in free flow using a microfluidic device. Lab on a Chip, 2003. 3(4): p. 224-227.

13. Albrecht, J.W. and K.F. Jensen, Micro free-flow IEF enhanced by active cooling and functionalized gels. Electrophoresis, 2006. 27(24): p. 4960-4969.

14. de Jesus, D.P., L. Blanes, and C.L. do Lago, Microchip free-flow electrophoresis on glass substrate using laser-printing toner as structural material. Electrophoresis, 2006. 27(24): p. 4935-4942.

15. Fonslow, B.R. and M.T. Bowser, Optimizing band width and resolution in micro-free flow electrophoresis. Analytical Chemistry, 2006. 78(24): p. 8236-8244. 
16. Albrecht, J.W., J. El-Ali, and K.F. Jensen, Cascaded free-flow isoelectric focusing for improved focusing speed and resolution. Analytical Chemistry, 2007. 79(24): p. 9364-9371.

17. Kohlheyer, D., J.C.T. Eijkel, S. Schlautmann, A. van den Berg, and R.B.M. Schasfoort, Microfluidic high-resolution free-flow isoelectric focusing. Analytical Chemistry, 2007. 79(21): p. 8190-8198.

18. Jacobson, S.C. and J.M. Ramsey, Electrokinetic focusing in microfabricated channel structures. Analytical Chemistry, 1997. 69(16): p. 3212-3217.

19. Mesaros, J.M., G. Luo, J. Roeraade, and A.G. Ewing, Continuous Electrophoretic Separations in Narrow Channels Coupled to Small-Bore Capillaries. Analytical Chemistry, 1993. 65(22): p. 3313-3319.

20. Mesaros, J.M. and A.G. Ewing, Characterization of Continuous Electrophoretic Separations in Narrow Channels Coupled to Small-Bore Capillaries. Journal of Microcolumn Separations, 1994. 6(5): p. 483-494.

21. Belder, D., M. Ludwig, L.W. Wang, and M.T. Reetz, Enantioselective catalysis and analysis on a chip. Angewandte Chemie-International Edition, 2006. 45(15): p. $2463-2466$.

22. Besselink, G.A.J., P. Vulto, R.G.H. Lammertink, S. Schlautmann, A. van den Berg, W. Olthuis, G.H.M. Engbers, and R.B.M. Schasfoort, Electroosmotic guiding of sample flows in a laminar flow chamber. Electrophoresis, 2004. 25(21-22): p. 3705-3711.

23. Ermakov, S.V., S.C. Jacobson, and J.M. Ramsey, Computer simulations of electrokinetic injection techniques in microfluidic devices. Analytical Chemistry, 2000. 72(15): p. 3512-3517.

24. Kohlheyer, D., G.A.J. Besselink, R.G.H. Lammertink, S. Schlautmann, S. Unnikrishnan, and R.B.M. Schasfoort, Electro-osmotically controllable multi-flow microreactor. Microfluidics and Nanofluidics, 2005. 1(3): p. 242-248.

25. Zalewski, D.R., S. Schlautmann, R.B.M. Schasfoort, and J.G.E. Gardeniers, Electrokinetic sorting and collection of fractions for preparative capillary electrophoresis on a chip. Lab on a Chip, 2008. 8(5): p. 801-809.

26. Kohlheyer, D., G.A.J. Besselink, S. Schlautmann, and R.B.M. Schasfoort, Free-flow zone electrophoresis and isoelectric focusing using a microfabricated glass device with ion permeable membranes. Lab on a Chip, 2006. 6(3): p. 374-380.

27. Crank, J., The mathematics of diffusion. 2 ed. 1975, Oxford: Clarendon Press. 



\section{Chapter VII \\ Fractionation of a Two-Component Mixture by SCFZE}

\footnotetext{
Ynchronized continuous-Flow zone electrophoresis $\mathcal{S}$ (SCFZE), introduced in chapter 6 , is a recently demonstrated tool for performing electrophoretic fractionation of a complex sample. The method resembles free-flow electrophoresis, but unlike in that technique, no mechanical fluid pumping is required. Instead, fast electrokinetic flow switching is used to produce complex stream patterns, which results in lateral separation of components in a separation chamber. Although collection of separated fractions is non-trivial and previous developments permitted pooling of one component only, we present here a solution which allows for simultaneous collection of two fractions. Moreover, in contrast with the previous design, the collected samples are not cross-contaminated. We demonstrate the performance of the method on a model mixture and evaluate fractionation efficiency by performing capillary electrophoresis of the collected fractions. We also provide the necessary theoretical background.
} 


\section{Introduction}

Separation science is one of the most active domains benefiting from miniaturization through chip formats. ${ }^{1-3}$ Among the many separation methods that have been downscaled to the microscale, continuous separation techniques have attracted significant attention. ${ }^{4}$ The main benefit of performing continuous separations is the possibility of processing a substantial amount of material per time unit, as compared to sequentially operating methods. This ability makes the continuous separation techniques excellent preparative tools. Fractionated components can usually be pooled in individual containers and later either further processed in an integrated system or extracted from a chip.

Electrophoresis is one of the most commonly used separation routines and a continuous separation based on this principle, known as free flow electrophoresis (FFE) was introduced already in $1961 .{ }^{5}$ During FFE a sample is introduced into a rectangular separation bed by mechanical pumping and an electric field is applied in the direction transverse to the flow, causing the components to separate. A miniaturized FFE device was first demonstrated by Raymond and co-workers in $1994 .{ }^{6}$ The topic was further researched, attracting growing interest over the past years, manifested by an increasing number of publications. ${ }^{7}$ Many chip devices have been reported, demonstrating different FFE separation modes, including: zone electrophoresis, ${ }^{8-11}$ isoelectric focusing, ${ }^{12-14}$ and isotachophoresis. ${ }^{15}$

Recently, we proposed a new way of performing continuous electrophoretic separation in a microfluidic system, named continuous-flow zone electrophoresis (CFZE). ${ }^{16}$ Unlike in FFE, running a CFZE separation does not require mechanical pumping; the flow in the device is induced electrokinetically. Moreover, in contrast to FFE, where the electric field is applied in the direction transverse to the flow, in CFZE separation of the components occurs in the flow direction; i.e. the same electric field that drives the flow also separates the sample. This makes the collection of separated fractions non-trivial and a special technique of coordinated switching of the inlet and outlet fluxes, called synchronized continuous-flow zone electrophoresis (SCFZE), must be employed to pool one selected component. Furthermore, the collection of a completely uncontaminated fraction is physically impossible and thus only tens-fold purification was achievable.

Here we present a method for performing SCFZE with simultaneous collection of two components. This improvement is possible by changing the design with respect to the earlier version of the device and introducing a new fluxes synchronization 
scheme. Moreover, this new approach enables pooling of pure fractions with virtually no contamination. We describe the technique theoretically and validate it in the experiments, showing additional important changes to the steering model that improves the collection efficiency. Finally, we assess the purity of the fractionated components by capillary electrophoresis of the extracted samples.

\section{Model of operation}

The principle of CFZE was described elsewhere, ${ }^{16}$ here we briefly summarize it. For the description of the separation process, we refer to Figure 1. A multicomponent sample mixture is continuously injected into the separation chamber through the middle inlet. Within the chamber, the sample is sandwiched by two sheath streams and forms a narrow stream travelling in longitudinal direction under the applied electric field $E_{S}$. The position of the sample stream at the beginning of the chamber is varied over time in a predefined way by changing the fluxes of the sheath streams. ${ }^{17}$ The periodic variations of the starting position of the sample stream combined with the axial electric field, present in the chamber, produce a wavelike sample stream path - in case of sinusoidal modulation of the starting position the path takes the theoretical form:

$$
y(x, t)=-A \sin \left(\omega\left(\frac{x}{\mu_{S} E_{S}}-t\right)\right)
$$

Where $A$ is the amplitude of the modulation, $\mu_{s}$ - the apparent electrokinetic mobility of the sample component, $\omega$ - the angular frequency of the position modulation, $x$ the distance in the axial direction in the chamber and $t$ indicates time. Since the wavelength of the sample stream depends on the apparent mobility of a sample, for complex mixtures consisting of components with different mobility, more than one wave is observed in the chamber - each wave belonging to a different fraction. Figure 1 shows a typical wave pattern for a two-component mixture. 


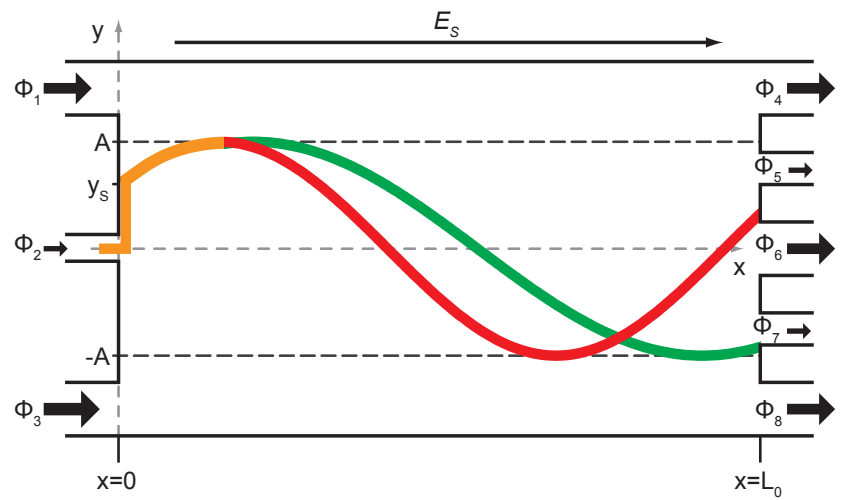

Figure 1. The principle of CFZE. Theoretical sample stream paths during CFZE of a twocomponent mixture.

Because the transverse position of the sample streams at the end of the chamber varies over time according to equation (1) as $y\left(L_{0}, t\right)$, where $L_{0}$ is the length of the chamber, the collection of the fractions is, unlike in free flow electrophoresis, not straightforward. The locations of the outlets cannot be varied to follow the changing stream position, instead switching of the outlet fluxes must be employed similar to the switching sequence at the entrance of the chamber. For the collection of one component only three outlets are needed and the flux variation pattern applied on the inlets is mirrored with an appropriate phase shift for the outlets. ${ }^{16}$

For simultaneous collection of two fractions a different strategy must be employed. The number of chamber outlets is increased to five. The outmost outlets are used as general purpose sheath stream outlets and the middle one is a waste sink. The two remaining outlets (5 and 7 in Figure 1) are utilized for collecting the separated fractions. The SCFZE cycle during which two components are simultaneously collected can be divided into two phases: the waste phase and the collection phase. The steering schemes for these phases differ significantly.

Consider a two-component mixture, consisting of component $A$ and component $B$, that is fractionated with SCFZE. For the purpose of this study, we assume that for the component A the sample outlet 5 was chosen as the collection channel and for the component $B$ the sample outlet 7 . The theoretical positions at the end of the chamber, $y_{A}\left(L_{0^{\prime}} t\right)$ and $y_{B}\left(L_{0^{\prime}} t\right)$, of both component streams vary over time according to equation (1) and thus two situations can be distinguished. The first, when $y_{A}\left(L_{0^{\prime}} t\right) \leq y_{B}\left(L_{0^{\prime}} t\right)$ - we refer to as the waste phase, and the second when $y_{A}\left(L_{0}, t\right)>y_{B}\left(L_{0}, t\right)$ - is the collection phase. Because the streams have finite widths, in real operation the 
discrimination between the waste and collection phases is done by testing the following condition

$$
y_{A}\left(L_{0}, t\right)-y_{B}\left(L_{0}, t\right) \leq c_{D} \cdot w_{\text {Sinj }}
$$

where $w_{\text {Sinj }}$ is the width of the injected sample stream and $c_{D}$ is an arbitrarily chosen discrimination coefficient.
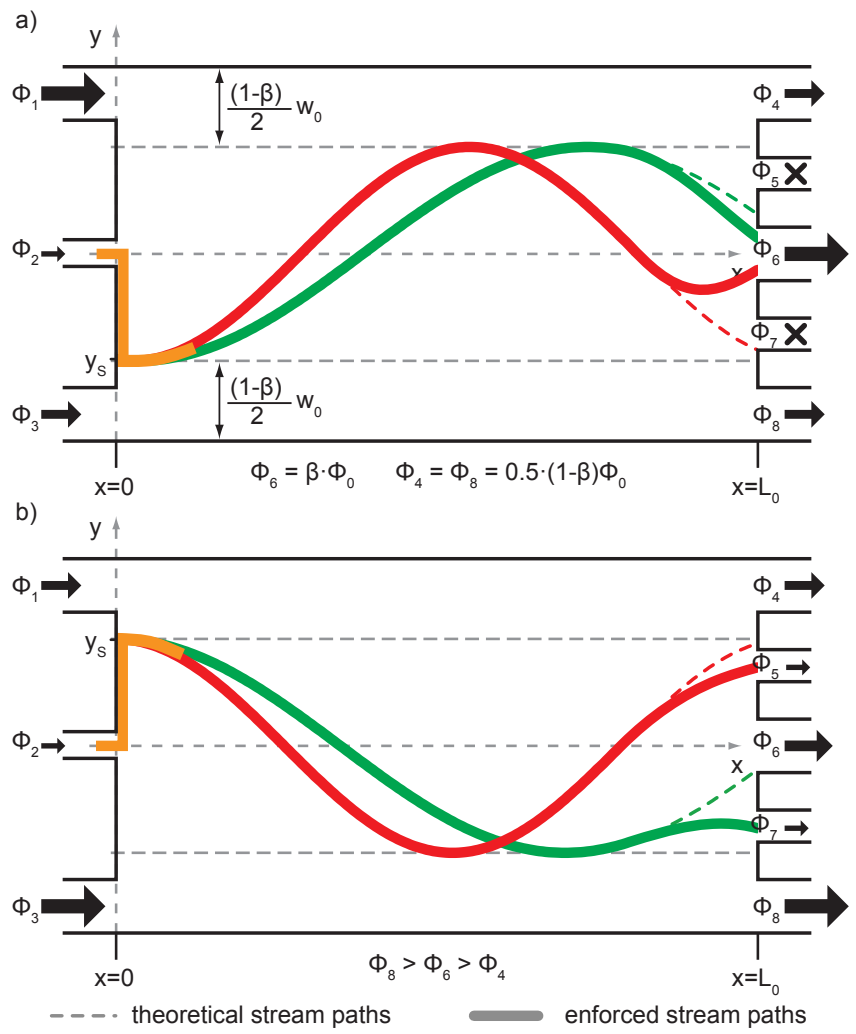

Figure 2. The principle of operation. (a) Guiding both streams into the waste outlet when components cannot be collected into their mutual outlets $-y_{A}\left(L_{0}, t\right) \leq y_{B}\left(L_{0}, t\right)$. (b) Simultaneous collection of both components by synchronized steering of outlet fluxes for time $t$ when $y_{A}\left(L_{0}, t\right)>y_{B}\left(L_{0}, t\right)$.

During the waste phase the exit positions of the component streams are reversed in reference to their designated sample outlet - the collection of the fractions is therefore physically impossible. However, to avoid contamination of the collection channels with incorrect components the fractions must be guided into other outlets. The most intuitive way to achieve this is to direct all the fluid flowing through the chamber to the sheaths outlets and the middle waste sink (Figure 2a). The ratio of these fluxes can be chosen arbitrary but the sum of them must equal the total 
inlet flux. The potentials that need to be applied to the channels can be obtained by applying a method used for the three-outlet SCZFE device. ${ }^{16}$

The collection phase, during which the sample streams at the exit of the chamber are positioned according their collection channels, requires a more elaborate steering scheme. In principle the system can be considered as two independently operated SCFZE separation devices with three outlets each, which share one sheath stream outlet. For the calculation of the fluxes during this phase the buffer conductivity $\sigma_{0}$ and mobility $\mu_{0}$ as well as detailed device geometry must be known. Also, the desired electric field strength of separation $E_{s}$ must be provided. We also define the following non-dimensional parameters: the sample stream width coefficient $\alpha$ - that is the width of the sample stream taken as a fraction of the chamber width; the sample wave confinement coefficient $\beta$ - understood as a fraction of the chamber width occupied by the sample wave (see Figure 2); $\delta$ - the sample diffusion coefficient - this number describes the ratio of the sample stream width at the end of the chamber to that at the beginning of the chamber, and is meant to compensate partly for the lateral diffusion of the stream. The following values are defined based on chip dimensions: the amplitude of the wave $A$

$$
A=\frac{\beta-\alpha}{2} w_{0}
$$

and the minimum sheath stream flux, that is the sheath flux that restricts the confinement of the sample wave within the limits set by wave confinement coefficient $\beta$

$$
\phi_{\min }=\frac{1-\beta}{2} \phi_{0}
$$

The total flux through the separation chamber is given by

$$
\phi_{0}=\mu_{0} E_{S} S_{0}
$$

where $S_{0}$ is the cross-sectional area of the chamber. Modification of the flux equations for the SCFZE device with three outlets yields the following 


$$
\begin{aligned}
& \phi_{1}(t)=\phi_{\min }+\frac{A-y_{s}(0, t)}{2 A}(\beta-\alpha) \phi_{0} \\
& \phi_{2}(t)=\alpha \phi_{0} \\
& \phi_{3}(t)=\phi_{\min }+\frac{A+y_{s}(0, t)}{2 A}(\beta-\alpha) \phi_{0} \\
& \phi_{4}(t)=\phi_{\min }+\frac{A-y_{A}\left(L_{0}, t\right)}{2 A}(\beta-\alpha \delta) \phi_{0} \\
& \phi_{5}(t)=\phi_{7}(t)=\alpha \delta \phi_{0} \\
& \phi_{6}(t)=\left[\frac{y_{A}\left(L_{0}, t\right)-y_{B}\left(L_{0}, t\right)}{2 A}(\beta-\alpha \delta)-\alpha \delta\right] \phi_{0} \\
& \phi_{8}(t)=\phi_{\min }+\frac{A+y_{B}\left(L_{0}, t\right)}{2 A}(\beta-\alpha \delta) \phi_{0}
\end{aligned}
$$

After defining the fluxes through all channels the corresponding electric currents can be obtained from

$$
i_{i}(t)=\phi_{i}(t) \frac{\sigma_{0}}{\mu_{0}}
$$

With all the currents known, it is straightforward to derive an equivalent electric circuit of the device ${ }^{18,19}$ and calculate, by using the Kirchhoff's laws, the differences between the potentials that must be applied to the channels. The lowest potential is assumed to equal zero and the remaining voltages are calculated.

\section{Experimental section}

\section{Microchip fabrication}

The chip was fabricated using standard microfabrication techniques. ${ }^{20}$ The device consisted of two, $1.1 \mathrm{~mm}$ thick, borosilicate glass plates. The top plate contained the fluidic channels etched $10 \mu \mathrm{m}$ deep in 10\% HF through a photolithography patterned $\mathrm{Cr} / \mathrm{Au}$ mask and reservoirs micro-powder blasted with $\mathrm{Al}_{2} \mathrm{O}_{3}$ particles. After stripping the masking material from the top plate and a cleaning step, the wafers were thermally bonded at $600{ }^{\circ} \mathrm{C}$. Afterwards the bonded stack was diced into $20 \mathrm{~mm}$ x $15 \mathrm{~mm}$ chip devices.

\section{Chemicals and procedures}

The chemicals were purchased from Sigma-Aldrich-Fluka. Stock solutions of $100 \mathrm{mM}$ MES/Histidine, $10 \mathrm{mg} \cdot \mathrm{mL}^{-1}$ rhodamine B and $10 \mathrm{mg} \cdot \mathrm{mL}^{-1}$ fluorescein were prepared and filtered through a $0.8 \mu \mathrm{m}$ membrane filter. The stock solutions were 
used to obtain $20 \mathrm{mM}$ MES/Histidine buffer and a sample mixture containing $500 \mu \mathrm{M}$ rhodamine B and $500 \mu \mathrm{M}$ fluorescein in this buffer. Subsequently the solutions were filtered with a $0.22 \mu \mathrm{m}$ membrane filter and degassed in a vacuum chamber for 15 minutes.

The chip was placed in a custom-made holder, which provided a flexible way of filling the reservoirs and connecting the voltage sources. Platinum electrodes submerged in buffer-filled reservoirs were used to connect the fluidic network to the voltage sources. Two high-voltage power supplies, with four independently operated channels each (CU-411, IBIS B.V., The Netherlands) were controlled by an in-house written C\# application with a frequency of $20 \mathrm{~Hz}$. An inverted fluorescence microscope (Olympus IX51) equipped with a 24 bit digital colour camera (ColorView II, Olympus) and a wide blue filter (11012v2, Chroma Technology, USA) was used for visualization.

Before the experiments, the holder reservoirs were filled with $20 \mu \mathrm{L}$ buffer solution each and the chip was flushed by inducing electroosmotic flow for 5 minutes. Afterwards visual inspection ensured lack of bubbles and obstacles in the chip fluidic network and the buffer in all but the sample source reservoirs was exchanged. The sample reservoir was filled with $20 \mu \mathrm{L}$ of the sample solution.

During the waste phase of synchronized collection the waste flux was set to the value of the sample wave confinement coefficient $\beta$, unless otherwise indicated.

\section{Results and discussion}

\section{Chip device}

Figure 3 shows a photograph of the fabricated chip device. The separation chamber dimensions were $4 \mathrm{~mm} \times 1.5 \mathrm{~mm}$. The depth of the whole fluidic network was $10 \mu \mathrm{m}$. All inlet and outlet channels consisted of two sections - a short and narrow part connected to the chamber and a much wider section following. The widening of the channels was used to reduce electrical resistance of the channels and thus enable higher separation fields to be used. The detailed dimensions of the channels are grouped in Table 1. 


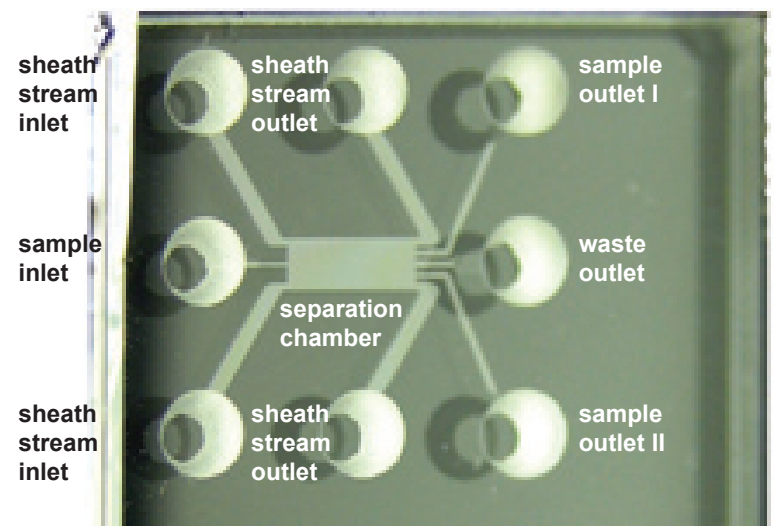

Figure 3. Photograph of a fabricated chip device with labelled inlets and outlets. The total dimensions of the chip were $20 \mathrm{~mm} \times 15 \mathrm{~mm}$.

Table 1. Dimensions of the channels of the SCFZE chip device.

\begin{tabular}{lll} 
& $\begin{array}{l}\text { Section I } \\
(\mathbf{L x W}) / \boldsymbol{\mu m}\end{array}$ & $\begin{array}{l}\text { Section II } \\
(\mathbf{L x W}) / \boldsymbol{\mu m}\end{array}$ \\
\hline Sheath inlet & $200 \times 200$ & $4120 \times 500$ \\
Sample inlet & $900 \times 100$ & $1180 \times 200$ \\
Sheath outlet & $200 \times 200$ & $4120 \times 500$ \\
Sample outlet & $400 \times 100$ & $4800 \times 200$ \\
Waste outlet & $1160 \times 200$ & $930 \times 500$
\end{tabular}

\section{Sample fractionation}

We started with performing CFZE of the sample mixture without synchronization of the outlet fluxes. Figure 4 shows example wave patterns obtained during the separation for $E_{S}=350 \mathrm{~V} \cdot \mathrm{cm}^{-1}, \omega=\pi \mathrm{rad}, \alpha=0.001$ and $\beta=0.6$. This step served two purposes: first it ensured a proper operation of the device, and secondly the fluorescence images taken during the separation were used to measure the wavelengths of both sample streams. With known separation field strength and angular modulation frequency the apparent mobility of the sample component can be calculated from the wavelength

$$
\mu_{S}=\frac{\lambda \omega}{2 \pi E}
$$

The average mobility of each component in the sample mixture, calculated by taking eight measurements for two different angular frequencies of modulation $\left(\omega_{1}=0.5 \cdot \pi \mathrm{rad}, \omega_{2}=0.025 \cdot \pi \mathrm{rad}\right)$ was: $\mu=8.42 \cdot 10^{-8} \mathrm{~m}^{2} \cdot(\mathrm{Vs})^{-1}$ for rhodamine $\mathrm{B}$ and $\mu=5.97 \cdot 10^{-8} \mathrm{~m}^{2} \cdot(\mathrm{Vs})^{-1}$ for fluorescein. These values were only estimates and they 
were later used during the synchronized collection as a starting point for finetuning of the synchronization parameters.

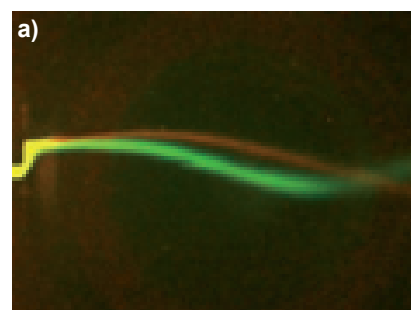

c)

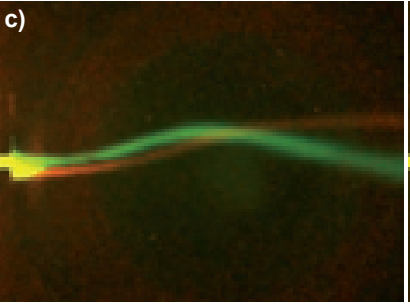

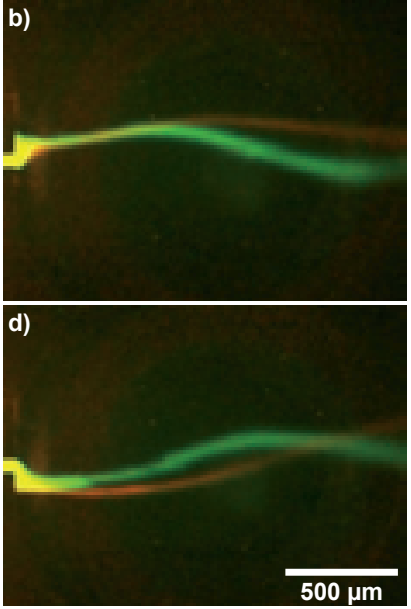

$500 \mu \mathrm{m}$

Figure 4. Continuous-flow zone electrophoresis of a rhodamine $\mathrm{B} /$ fluorescein mixture with the separation field strength $E_{s}=350 \mathrm{~V} \cdot \mathrm{cm}^{-1}$. The wavelength of the rhodamine $B$ wave (red) is greater than that of the fluorescein wave (green) because rhodamine $B$ has a larger apparent mobility. The time span between consecutive frames is $600 \mathrm{~ms}$.

Figure 5 shows a sequence of images taken during the synchronized collection of the fractions with an erroneous synchronization pattern observed during the search for the correct mobility values. The operating parameters for this run were: $E_{S}=350 \mathrm{~V} \cdot \mathrm{cm}^{-1}, \omega=0.5 \cdot \pi \mathrm{rad}, \alpha=0.05, \beta=0.5, \delta=1$ and $c_{D}=1$. The mobility values of the components were set in the steering script to the following values: $\mu_{A}=6 \cdot 10^{-8} \mathrm{~m}^{2} \cdot(\mathrm{Vs})^{-1}$ and $\mu_{B}=7 \cdot 10^{-8} \mathrm{~m}^{2} \cdot(\mathrm{Vs})^{-1}$. Consequently, this resulted in invalid collection of the fractions; the waste phase of the synchronization cycle is visible in Figure 5a,f - both component streams entered the waste outlet as predicted. Yet, during the collection phase, shown in Figure 5b-e, the fraction streams swept unpredictably over the whole chamber width, entering nearly all possible outlets and leaving traces of contamination in both sample collection channels. The large broadening of the streams, which increases as the stream approaches the end of the chamber during the collection phase (compare e.g. the green fluorescein stream in Figure $5 \mathrm{a}$ and $5 \mathrm{~b}$ ) is mainly caused by the synchronization steering scheme. The manipulation of the outlet stream fluxes causes focusing and defocusing of the streams, depending on the position in the chamber and time. This, combined with diffusion causes the visual effect of rapid stream broadening. 

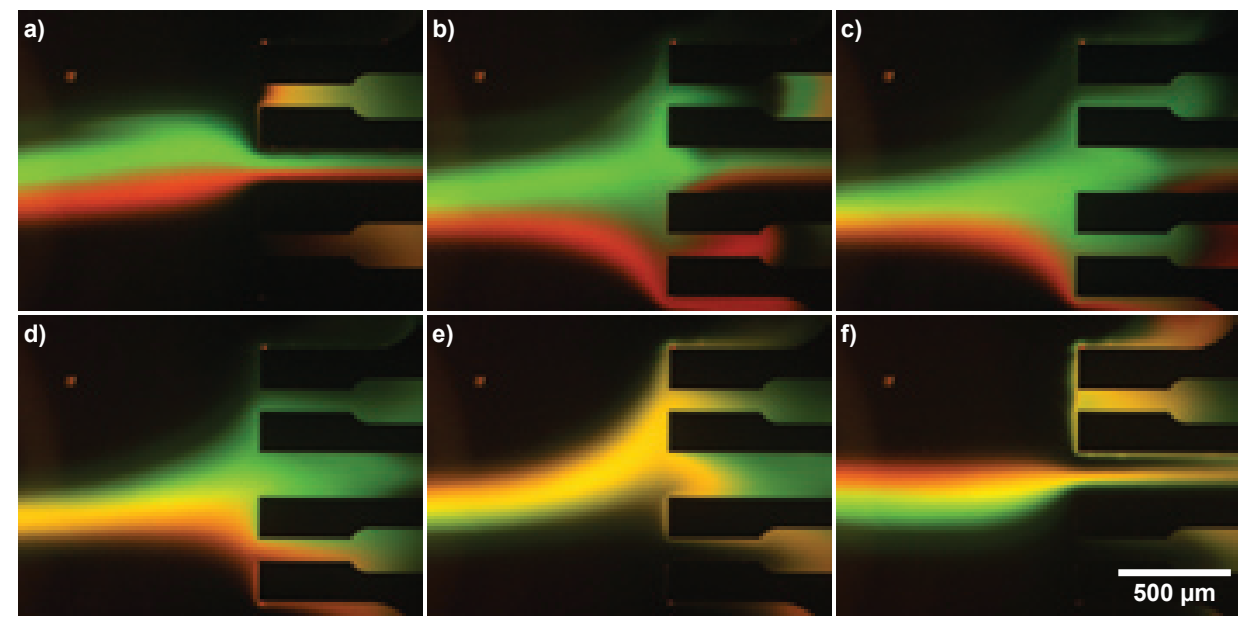

Figure 5. Collection of separated components with wrong synchronization pattern. Both streams enter various outlets and thus no fractionation occurs. The time span between consecutive frames is $\mathbf{4 0 0} \mathrm{ms}$.

By changing the component mobilities in the steering program in small steps of $\Delta \mu=0.05 \cdot 10^{-8} \mathrm{~m}^{2} \cdot(\mathrm{Vs})^{-1}$ and fine-tuning them, we found the values: $\mu_{A}=6.56 \cdot 10^{-8} \mathrm{~m}^{2} \cdot(\mathrm{Vs})^{-1}$ and $\mu_{B}=8.27 \cdot 10^{-8} \mathrm{~m}^{2} \cdot(\mathrm{Vs})^{-1}$, for which the collection was visually satisfactory - that is, the contamination of the collection channels by the non-matching component couldn't be observed and the collection phase occurred properly. Both values differ from the estimations given before, which can be partly accounted for by errors in measurements and changes in separation conditions caused by the long operation - the device had already been continuously used for over 60 minutes. Figure 6 shows a sequence of fluorescence images taken during the synchronized collection of two components for following operating parameters: $E_{S}=350 \mathrm{~V} \cdot \mathrm{cm}^{-1}, \omega=0.5 \cdot \pi \mathrm{rad}, \alpha=0.05, \beta=0.5, \delta=1$ and $c_{D}=2$. The sequence starts with an image taken before the start of collection (Figure 6a) - the fractions were still overlapping, however in the next image (Figure 6b) the separation between the streams increased and what followed was the collection phase (Figure 6c-f) during which the streams were injected into their designated collection channels. The waste phase (Figure $6 \mathrm{a}, \mathrm{b}, \mathrm{g}-\mathrm{l}$ ) lasted much longer than the collection - this was due to relatively wide sample stream $(\alpha=0.05)$, which combined with the discrimination coefficient $c_{D}=2$ and narrow wave confinement made the overlapping condition (2), valid throughout the majority of the cycle duration. 

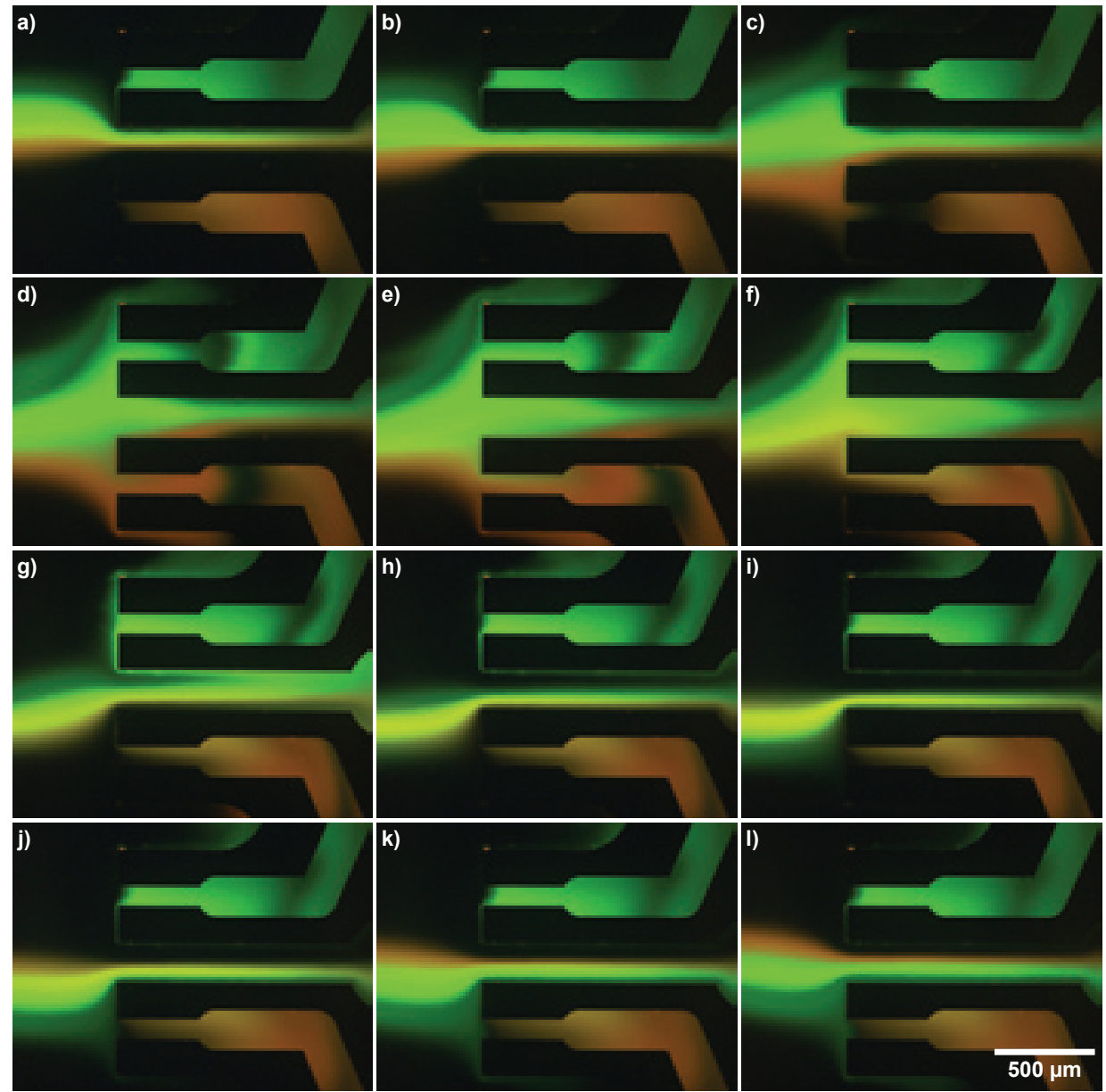

Figure 6. Sequence of fluorescence images taken during synchronized continuous-flow zone electrophoresis of rhodamine B / fluorescein mixture with simultaneous collection of both fractions. Almost a whole synchronization cycle is visualized the collection is shown in c-f; on the remaining images the separation between the streams is not large enough to make contamination-free collection $(a, b$, $g, h)$ or the streams positions do not permit switching: $y_{A}\left(L_{0}, t\right)<y_{B}\left(L_{0}, t\right)$, and thus both fractions are guided into the waste channel. The time span between consecutive frames is $\mathbf{4 0 0} \mathrm{ms}$.

In Figure 6i-l, there is a visible broadening of the fluorescein stream, a part of it expanded towards the lower collection channel - the situation lasted for a remarkable amount of time: 1.2 seconds and threatened the purity of the collected rhodamine B fraction. The broadening is caused by the already discussed phenomena - in this case the reason is the lower sheath stream, which drags a portion of the fluorescein stream and pushes it downwards in the direction of the sheath stream outlet. Such 
behaviour could lead to serious contamination if the sample outlet channels were not properly biased.

In the theoretical section, we discussed that during the waste phase the sample outlets can be electrically floating. Yet, we found that such an approach led to massive contamination of the sample outlet channels during the waste phase. To prevent this from happening, electric biasing of the sample outlets was used. The magnitude of the compensation voltage that needed to be applied to counteract the mechanism of contamination described above cannot be easily calculated analytically. Therefore instead of using a fixed number, this voltage was manually varied during the experiments by an operator until a proper value was found. In our experiments the usual biasing voltage applied to the sample outlet reservoirs was in the range of $(1.2 \div 1.6) \cdot \mathrm{U}_{\mathrm{C} 0}$, where $\mathrm{U}_{\mathrm{C} 0}$ was a theoretical potential at the end of the chamber (i.e. for $\mathrm{x}=\mathrm{L}_{0}$ ) calculated with the model. Figure 7 shows the behaviour of the stream during the waste phase for different sample outlet biasing scenarios. It can be noted that setting the bias voltage to a proper value can have a significant influence on the quality of fractionation (Figure $7 c, d$ - contamination due to underbiasing) and the quantity of collected material (Figure 7e,f - collected sample leak due to overbiasing).
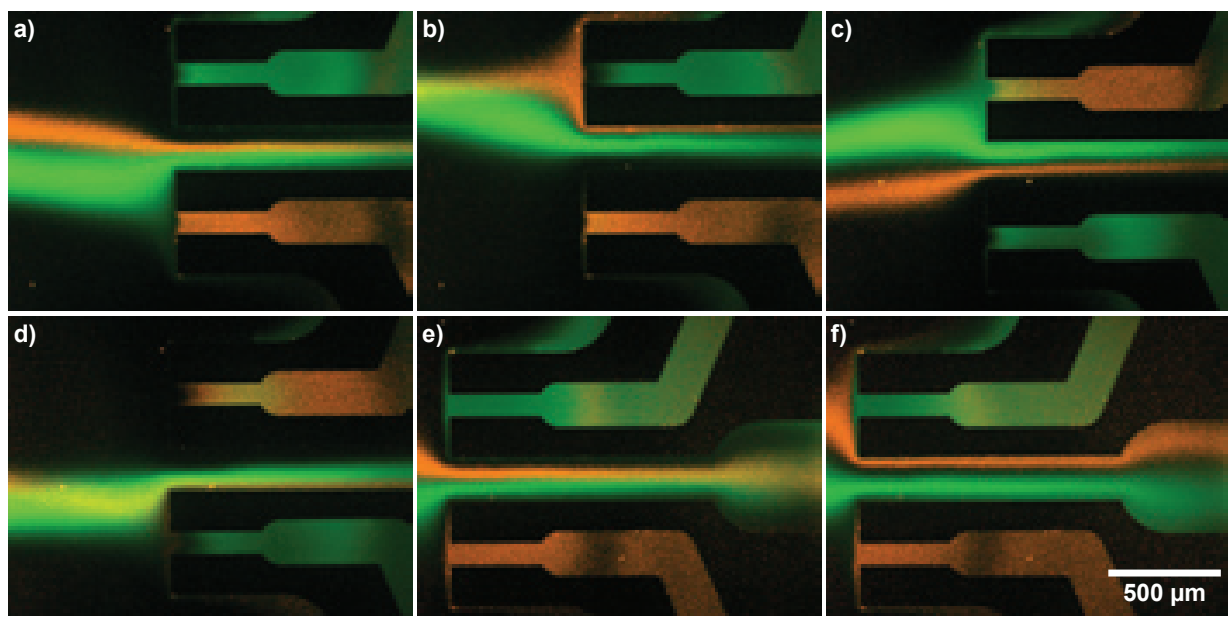

Figure 7. Fluorescence images of electrokinetic shielding of the collection channels in SCFZE during non-collection phase. $(a, b)$ Proper biasing - the sample streams do not contaminate the collection channels. (c,d) Underbiasing - the sample streams penetrate into the collection channels of the other fraction. $(e, f)$ Overbiasing - too much voltage is applied to collection channels causing leakage from the collection channels into the separation chamber and loss of already collected sample. 


\section{CE of fractionated sample}

After performing the SCFZE of the sample mixture for 15 minutes, under conditions described above, the fractionation was stopped. To assess the quality of fractionation the collected sample from the sample outlet reservoirs and from the waste reservoirs were extracted. Figure 8 shows fluorescence images of the reservoirs taken before the retrieval of the stored fluids. The collection reservoirs showed different fluorescence colours corresponding to the fractions they contained.
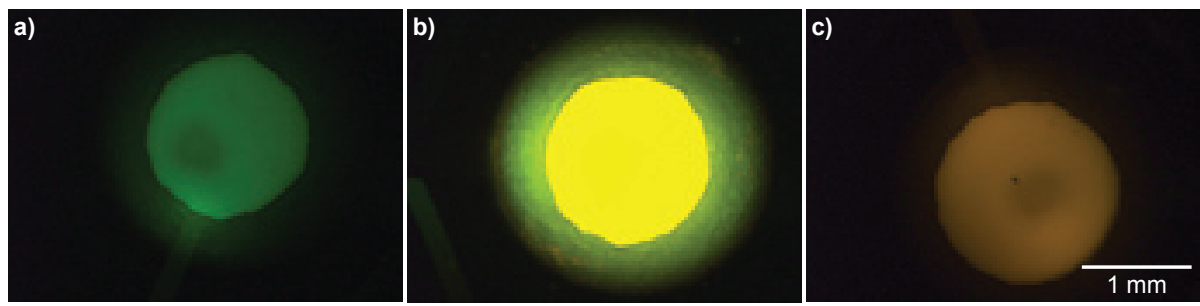

Figure 8. Fluorescence images of the collection $(a, c)$ and waste $(b)$ reservoirs after running the SCFZE of rhodamine B / fluorescein mixture for 15 minutes.

The extracted samples were transferred into a capillary electrophoresis chip. The CE chip was made of borosilicate glass and the separation channel was $100 \mu \mathrm{m}$ wide and $10 \mu \mathrm{m}$ deep. The $20 \mathrm{mM}$ MES/Histidine buffer was utilized as a running electrolyte and the separation field strength was set to $E_{s}=870 \mathrm{~V} \cdot \mathrm{cm}^{-1}$. Gated injections were used to introduce the sample into the separation channel with the injection time $t=1 \mathrm{~s}$. The PMT detector was located over the separation channel at a distance of $8.3 \mathrm{~mm}$ from the injection point.

Figure 9 shows the electropherograms registered during five consecutive separations of the samples extracted from the sample outlet reservoirs and the waste reservoir. The noise observed in the electropherograms was caused by the high sensitivity setting of the detector - the extracted samples were highly diluted and thus showed little fluorescence. The calculated dilution of the collected fraction due to buffer abundance in the outlet reservoirs was 75-fold. Clearly, the waste reservoir contained both rhodamine B and fluorescein fractions in amounts greater than the sample reservoirs. Yet, the sample outlets captured almost pure fractions of both rhodamine B and fluorescein, showing only very little contamination at the noise level. 


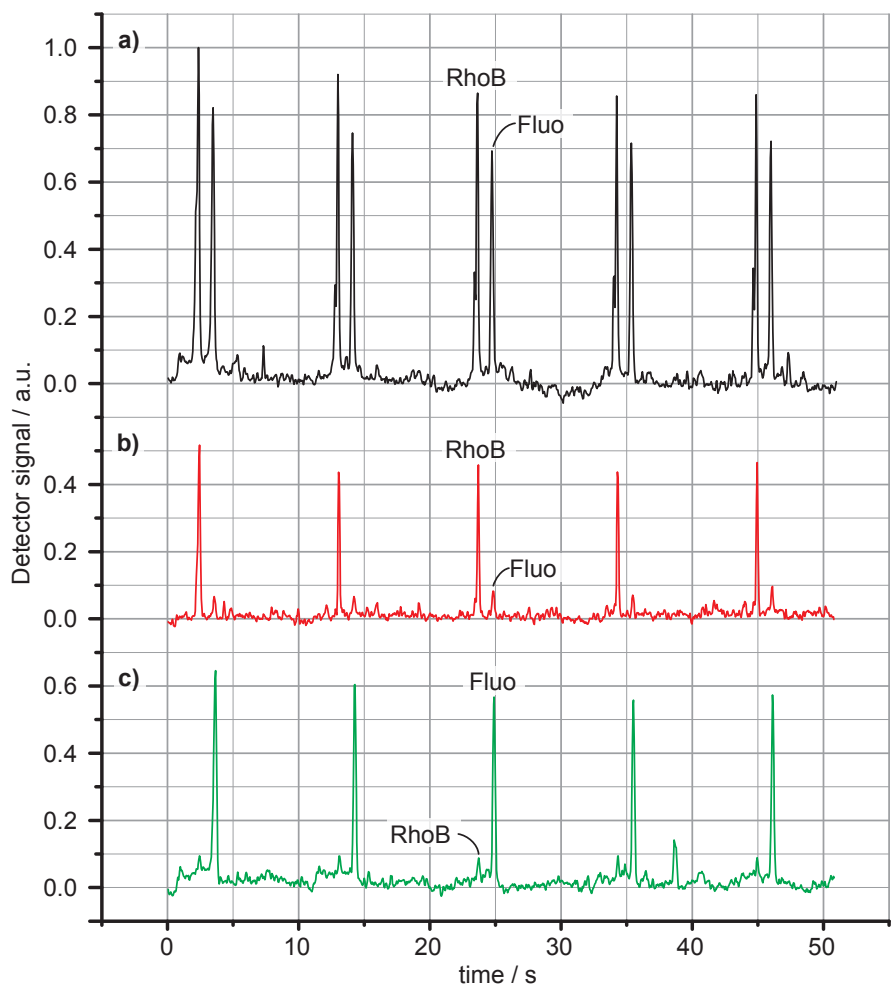

Figure 9. Electropherograms registered during the CE of the sample retrieved from the waste reservoir (a), rhodamine B collection reservoir (b) and fluorescein collection reservoir (c). The sample was collected during the SCFZE of the mixture containing both components over a period of 15 minutes. The dilution of the sample after the collection was substantial because of relatively big reservoir volumes $(20 \mu \mathrm{L})$.

\section{Performance measures of fractionation}

Theoretically, contamination of the collected fraction can be even further reduced by choosing greater values of the discrimination coefficient $c_{D}$. However, since increasing $c_{D}$ shrinks the duration of the collection phase, this could also affect the amount of collected material. To evaluate possible gains and losses of varying the discrimination coefficient, we performed a series of numerical computations in Matlab 7. The masses of both fractions collected by the sample outlets were computed by evaluating the integral

$$
m=\int_{T_{\text {col }}} \int_{y_{L}}^{y_{H}} C\left(L_{0}, y, t\right) d y d t
$$

where $T_{c o l}$ is the collection phase period, $\mathrm{C}_{\mathrm{i}}\left(\mathrm{L}_{0}, \mathrm{y}, \mathrm{t}\right)$ is the concentration function of 
the sample stream ${ }^{21}$ and $y_{L^{\prime}} y_{H}$ denote appropriate integration limits for the outlets. Subsequently the following measures were calculated: collection efficiency - the ratio of the collected mass to the total mass injected into the chamber for a given fraction

$$
E_{i}=\frac{m_{i i}}{M_{i}}
$$

and the fractionation selectivity computed as

$$
S_{i}=\frac{m_{i i} \cdot M_{j}}{M_{i} \cdot m_{j i}}
$$

Here $m_{i i}$ denotes the mass of the $i$-th fraction collected in the $i$-th sample outlet channel (i.e. its designated collection channel); $M_{i}$ and $M_{j}$ are the total masses injected into the separation chamber of the $i$-th and $j$-th fraction respectively; and $m_{j i}$ is the mass of the $j$-th fraction collected in the $i$-th sample outlet (i.e. the mass of contaminant in the collected $i$-th sample). Figure 10 shows the results of calculations for varying amplitude modulation frequency and different values of the discrimination coefficient $c_{D}$. For the computation, the remaining parameters were set to the values used in the fractionation experiment.

As can be seen in Figure 10a the selectivity of fractionation at the modulation frequency used in the experiments can be easily increased 75 times for the fluorescein fraction and 45 times for the rhodamine $\mathrm{B}$ fraction by using the discrimination coefficient $c_{D}=2.5$. In the same time the collection efficiency drops by $25 \%$ for both fractions (Figure 10b). Shifting the modulation frequency $\omega$ towards higher values boosts the selectivity even further, simultaneously increasing the mass of the collected sample.

It should be noted that even when working with low discrimination coefficients the selectivity of fractionation is still much higher than in the previously presented device. ${ }^{16}$ Under the fractionation conditions used in the experiments presented here, the selectivity for both fractions equalled roughly $S=17.5$. With the same conditions applied in the earlier device the selectivity would reach a maximum value of $S=6.4$ - a number almost three times smaller. 

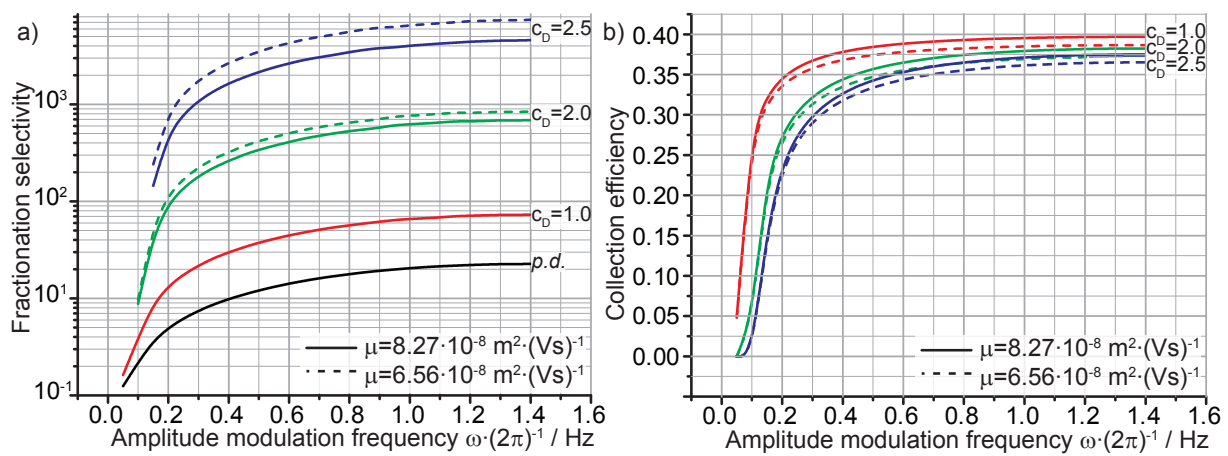

Figure 10. Plots of fractionation selectivity (a) and collection efficiency (b) for different values of the discrimination coefficient and varying amplitude modulation frequency. For comparison the selectivity of fractionation under same conditions in the previous SCFZE device is shown (p.d. in (a)). If only one line is visible for a given discrimination coefficient, the plots for both fractions overlap.

\section{Conclusions}

In conclusion, we proposed a method for performing synchronized, continuousflow zone electrophoresis with simultaneous collection of two components. The technique allows for electrophoretic continuous fractionation in an electrokinetic system, with virtually no cross-contamination of the collected samples. The method was successfully experimentally validated and the purity of the pooled fractions assessed by CE was good. Theoretical calculations show that further improvement of the fractionation quality is possible.

\section{Symbols}

$E_{S}$ - electric field throughout the separation chamber

$\mathrm{y}_{\mathrm{s}}$ - starting position of a sample stream

$A$ - amplitude of a sample stream wave

$\omega$ - angular frequency of oscillation

$\mu_{S}-$ apparent mobility of a sample component

$t$ - time

$y(x, t)$ - shape function of a sample stream

$L_{0}$ - length of a separation chamber

$\mu_{A}-$ apparent mobility of a sample component collected into the upper outlet

$\mu_{B}-$ apparent mobility of a sample component collected into the lower outlet 
$c_{D}-$ discrimination coefficient used to distinguish between the collection and waste phases

$\sigma_{0}-$ conductivity of a running electrolyte

$\mu_{0}-$ mobility of a running electrolyte

$\alpha-\quad$ ratio of sample stream width and chamber width

$\beta$ - sample confinement coefficient (i.e. a fraction of the chamber width, where a sample wave is present

$\delta$ - sample stream diffusion compensation coefficient - the ratio between the width of the sample stream at the end of the chamber to at the beginning of the chamber

$\phi_{0}-$ total flux through a chamber

$\phi_{1,3}$ - inlet sheath fluxes

$\phi_{4,8}$ - outlet sheath fluxes

$\phi_{2}-$ inlet sample flux

$\phi_{5,7}$ - outlet sample flux

$\phi_{6}-$ outlet waste flux

$w_{0}$ - width of a separation chamber

$\phi_{\min }-$ minimum sheath stream flux

E - efficiency of collection - a measure characterizing yield of collection

S - selectivity of fractionation - a measure characterizing quality of fractionation

$m_{i i}$ - mass of fraction collected into its designated reservoir

$m_{i j}$ - mass of fraction collected into incorrect reservoir

$M_{i}$ - total mass of fraction injected into a separation chamber

\section{References}

1. West, J., M. Becker, S. Tombrink, and A. Manz, Micro total analysis systems: Latest achievements. Analytical Chemistry, 2008. 80(12): p. 4403-4419.

2. Dittrich, P.S., K. Tachikawa, and A. Manz, Micro total analysis systems. Latest advancements and trends. Analytical Chemistry, 2006. 78(12): p. 3887-3907.

3. Vilkner, T., D. Janasek, and A. Manz, Micro total analysis systems. Recentdevelopments. Analytical Chemistry, 2004. 76(12): p. 3373-3385.

4. Pamme, N., Continuous flow separations in microfluidic devices. Lab on a Chip, 2007. 7(12): p. 1644-1659. 
5. Hannig, K., Die Tragerfreie Kontinuierliche Elektrophorese Und Ihre Anwendung. Fresenius Zeitschrift Fur Analytische Chemie, 1961. 181: p. 244-274.

6. Raymond, D.E., A. Manz, and H.M. Widmer, Continuous Sample Pretreatment Using a Free-Flow Electrophoresis Device Integrated onto a Silicon Chip. Analytical Chemistry, 1994. 66(18): p. 2858-2865.

7. Kohlheyer, D., J.C.T. Eijkel, A. van den Berg, and R.B.M. Schasfoort, Miniaturizing freeflow electrophoresis - a critical review. Electrophoresis, 2008. 29(5): p. 977-993.

8. Fonslow, B.R. and M.T. Bowser, Free-flow electrophoresis on an anodic bonded glass microchip. Analytical Chemistry, 2005. 77(17): p. 5706-5710.

9. Kobayashi, H., K. Shimamura, T. Akaida, K. Sakano, N. Tajima, J. Funazaki, H. Suzuki, and E. Shinohara, Free-flow electrophoresis in a microfabricated chamber with a micromodule fraction separator - Continuous separation of proteins. Journal of Chromatography A, 2003. 990(1-2): p. 169-178.

10. Zhang, C.X. and A. Manz, High-speed free-flow electrophoresis on chip. Analytical Chemistry, 2003. 75(21): p. 5759-5766.

11. Kohlheyer, D., G.A.J. Besselink, S. Schlautmann, and R.B.M. Schasfoort, Free-flow zone electrophoresis and isoelectric focusing using a microfabricated glass device with ion permeable membranes. Lab on a Chip, 2006. 6(3): p. 374-380.

12. Xu, Y., C.X. Zhang, D. Janasek, and A. Manz, Sub-second isoelectric focusing in free flow using a microfluidic device. Lab on a Chip, 2003. 3(4): p. 224-227.

13. Lu, H., S. Gaudet, M.A. Schmidt, and K.F. Jensen, A microfabricated device for subcellular organelle sorting. Analytical Chemistry, 2004. 76(19): p. 5705-5712.

14. Kohlheyer, D., J.C.T. Eijkel, S. Schlautmann, A. van den Berg, and R.B.M. Schasfoort, Microfluidic high-resolution free-flow isoelectric focusing. Analytical Chemistry, 2007. 79(21): p. 8190-8198.

15. Janasek, D., M. Schilling, J. Franzke, and A. Manz, Isotachophoresis in free-flow using a miniaturized device. Analytical Chemistry, 2006. 78(11): p. 3815-3819.

16. Zalewski, D.R., D. Kohlheyer, S. Schlautmann, and H.J.G.E. Gardeniers, Synchronized, Continuous-Flow Zone Electrophoresis. Analytical Chemistry, 2008.

17. Besselink, G.A.J., P. Vulto, R.G.H. Lammertink, S. Schlautmann, A. van den Berg, W. Olthuis, G.H.M. Engbers, and R.B.M. Schasfoort, Electroosmotic guiding of sample flows in a laminar flow chamber. Electrophoresis, 2004. 25(21-22): p. $3705-3711$. 
18. Berli, C.L.A., Theoretical modelling of electrokinetic flow in microchannel networks. Colloids and Surfaces a-Physicochemical and Engineering Aspects, 2007. 301(13): p. 271-280.

19. Berli, C.L.A., Equivalent circuit modeling of electrokinetically driven analytical microsystems. Microfluidics and Nanofluidics, 2008. 4(5): p. 391-399.

20. Zalewski, D.R., S. Schlautmann, R.B.M. Schasfoort, and H.J.G.E. Gardeniers, Electrokinetic sorting and collection of fractions for preparative capillary electrophoresis on a chip. Lab on a Chip, 2008. 8(5): p. 801-809.

21. Crank, J., The mathematics of diffusion. 2 ed. 1975, Oxford: Clarendon Press. 


\section{Chapter VIII \\ Conclusions and Outlook}

\footnotetext{
Certainly, the potential of electrokinetic flow control

$\checkmark$ for micropreparative electrophoresis on a chip was demonstrated in the previous chapters. The topic of performing micropreparative CE in chip devices slowly attracts attention of other groups. ${ }^{1,2}$ Yet, numerous problems remain unsolved - here some of them are addressed.
} 


\section{Hardware performance}

One of the main difficulties, briefly announced in the introduction to this thesis, is related to the control of external voltage sources. The time that is needed to switch the potential applied to the outlets of a microfluidic chip is still often counted in hundreds of milliseconds, yielding undesirable effects, and the exact reasons for such behaviour cannot be precisely identified. To give an example: a delayed system response can ruin the splitting of fractions (Figure 1). There exist devices on the market, that promise much faster responses (e.g. LabSmith, USA). However the advertised switching times are only achievable when an internal processor of the power supply is pre-programmed with a steering sequence - an approach certainly not applicable in actively controlled microfluidic systems.

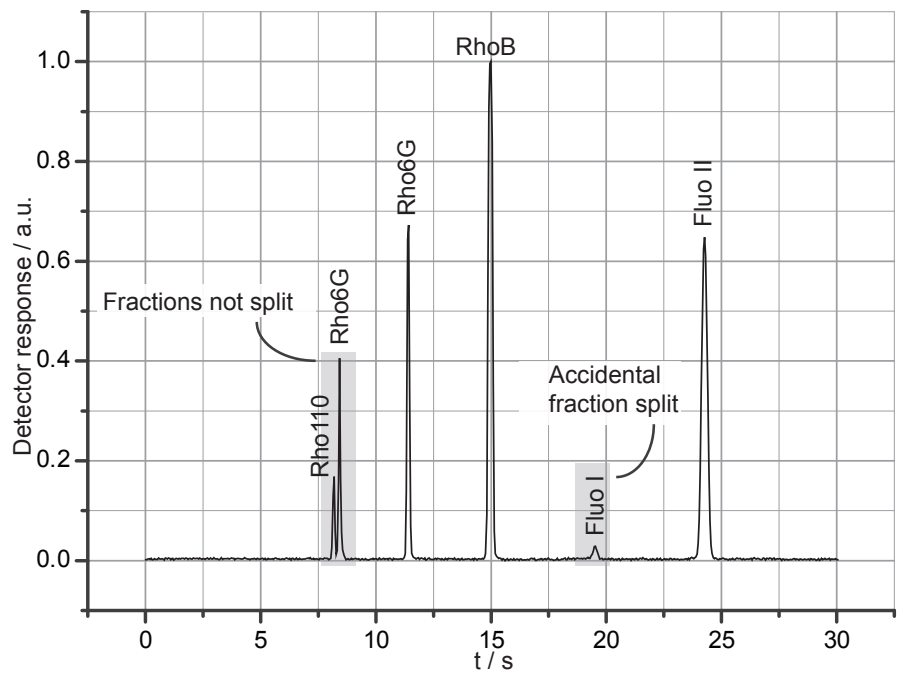

Figure 1. Typical errors encountered during fractions splitting in preparative CE chip.

\section{Junctions optimization}

Another problem frequently addressed throughout this thesis is the dispersion of analyte bands during passing of channel junctions. Not much research has been published on this topic - usually merely an identification of this phenomenon is claimed without any further studies. ${ }^{3}$ One working approach, besides precise junction biasing, is the integration of in-junction electrodes that can locally shape the electric field and thus minimize dispersion. ${ }^{4}$ However, this method is extremely demanding, as it involves additional fabrication steps and complicated steering logic. 
Li et al. showed that, alternatively, changing the junction geometry can seriously reduce the plug dispersion during manipulation. ${ }^{1}$ Interestingly, simulations seem to confirm the applicability of this approach to the peak splitting technique. As discussed in chapter 4 , the main concern during the splitting is not what happens to the band that passes the junction - since biasing of the side-channel can be applied during this process - but rather the dispersion that occurs to the awaiting fraction left behind in the separation channel.

Even a simple change of the junction type from a T-junction to a cross-junction seems to greatly reduce the dragging of the idle fraction and relax the magnitude of the pull-back biasing. Figure 2 shows simulations of concentration profiles of two, initially $25 \mu \mathrm{m}$ wide, sample plugs at $100 \mathrm{~ms}$ after starting the splitting, for both junction types. Identical potential configurations were used in the simulations. Using a symmetrical junction arrangement seems to greatly reduce the dragging of the idle plug. Needless to say, such an approach simplifies the steering schemes and contributes to the overall gain in splitting quality.

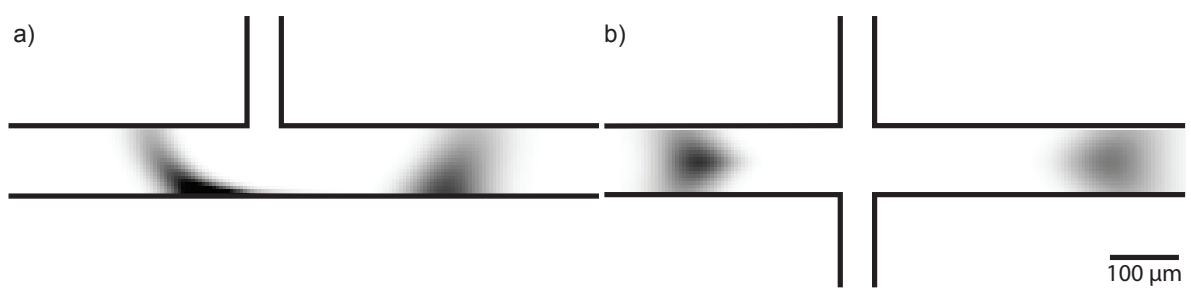

Figure 2. Simulations of $2 \mathrm{D}$ concentration profiles of fractions splitting for (a) a T-junction and (b) a cross-junction.

Also modifying the geometry of a T-junction can help reducing the dispersion. Figure 3 shows a comparison of simulations of equipotential lines for unbiased splitting in a straight and a modified T-junction. The use of the modified junction reduces the magnitude and the penetration distance of the electric field upstream the main channel, where the idle fractions reside.

Combination of both techniques could possibly lead to dismissal of the pull-back technique in most splitting scenarios. The gain in dispersion counteraction could be even greater because the idle fractions would not move upstream the separation channel during the transport of the split fraction and thus some time could be saved, normally needed to reposition them back near the junction. Any additional second gained this way would reduce the diffusional dispersion. 

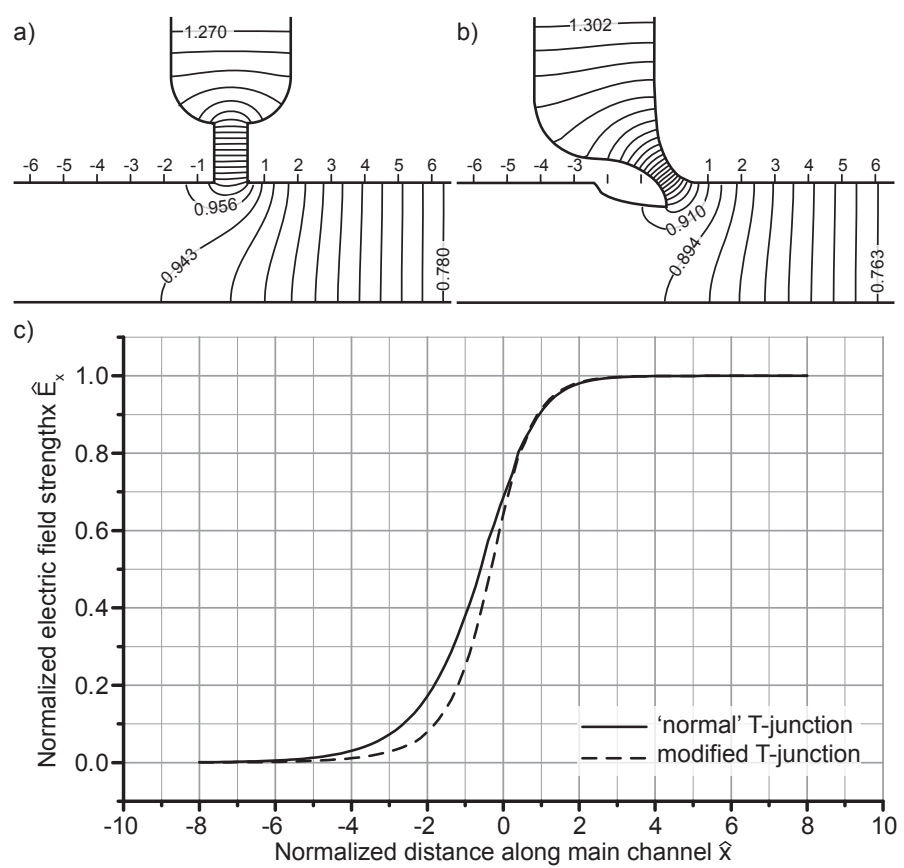

Figure 3. Simulations of equipotential lines at (a) a traditional T-junction; (b) modified T-junction geometry. (c) Electric field magnitude along the main channel for both junction geometries.

\section{Electric field in SCFZE}

The electric field uniformity is also a great concern in continuous-flow zone electrophoresis described in chapters 6 and 7. Figure 4 shows a fluorescence photograph of a sample stream near the exit of the SCFZE chamber. The end of the wave, in the case depicted, should be positioned in the vicinity of the upper sheath outlet if no synchronized steering was applied. Because of the conditions enforced by the outlet fluxes configuration, the wave sharply deflects towards the middle sample outlet. Such a situation obviously cannot be achieved with the uniform electric field in the chamber, as assumed in the theoretical description of SCFZE. 


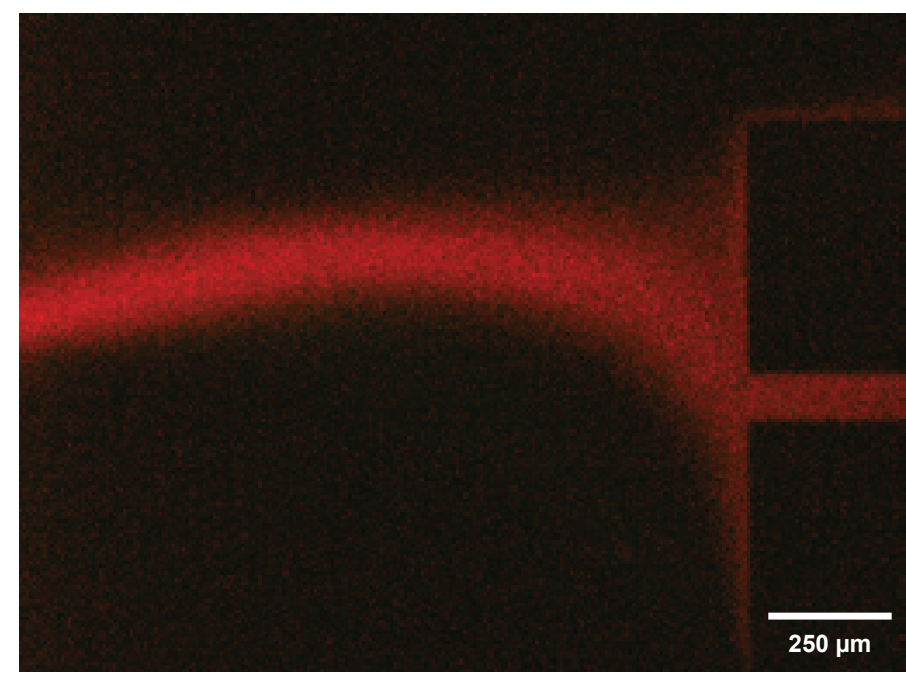

Figure 4. Maximum deflection of the rhodamine $B$ wave at the chamber exit.

Figure 5 shows simulations of equipotential lines and streamlines in the separation chamber for a case when the transverse starting position of the stream $y_{S}=0$ and for the maximum deflection case $y_{S}=A$. In both situations the electric field is not even nearly-uniform in the regions extending to over $500 \mu \mathrm{m}$ away from the chamber entrance and exit.

Detailed plots of the electric field magnitude in the separation chamber for both situations are shown in Figure 6. Obviously, such field distribution affects the sample wave shape (depicted as a blue line in Figure 4) and its influence grows with the increasing modulation amplitude. Figure 7 shows fluorescence photographs of sample waves obtained for extreme modulation amplitudes ( $\beta=0.8 . .0 .9)$, when the effects of electric field non-uniformity are most pronounced. The patterns that are observed are certainly interesting from the fluid mechanics theoretician point of view but completely ruin the fractionation because the prediction of the wave positions at the end of the separation chamber becomes virtually impossible. 

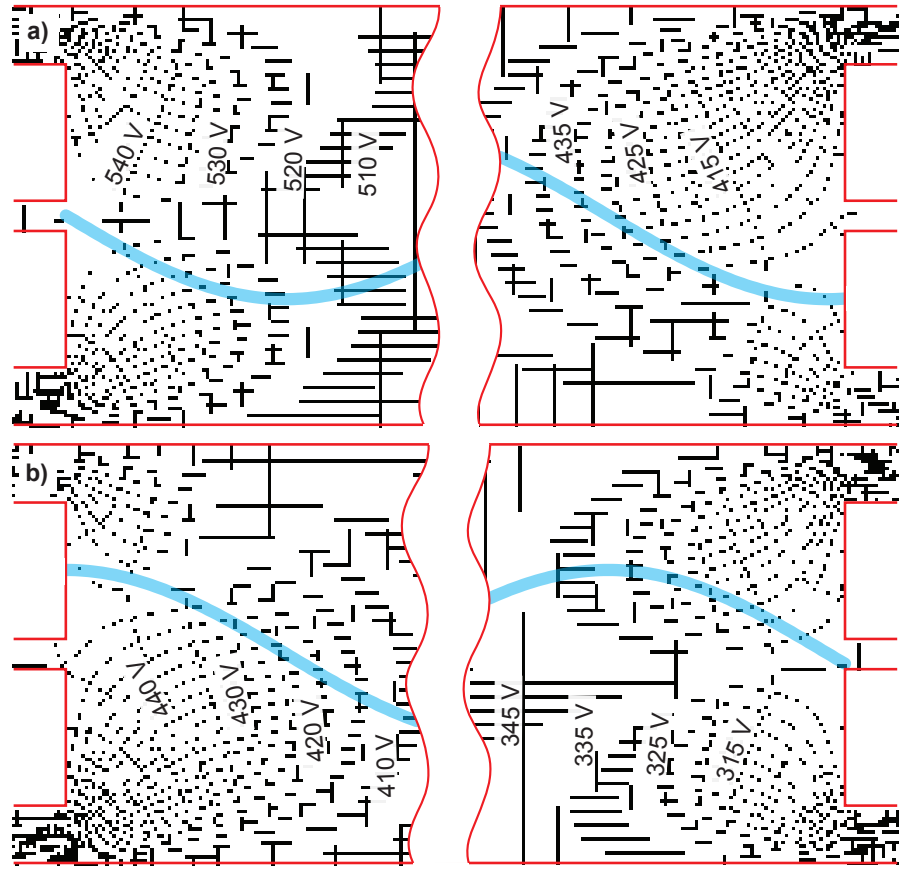

Figure 5. Simulations of streamlines and equipotential lines in a SCFZE device. The cases of the minimum deflection at the chamber entrance (a) and the maximum deflection at the chamber entrance (b) for the wave confinement coefficient $\beta=0.4$ are shown. (The blue line indicates theoretical sample wave path.)
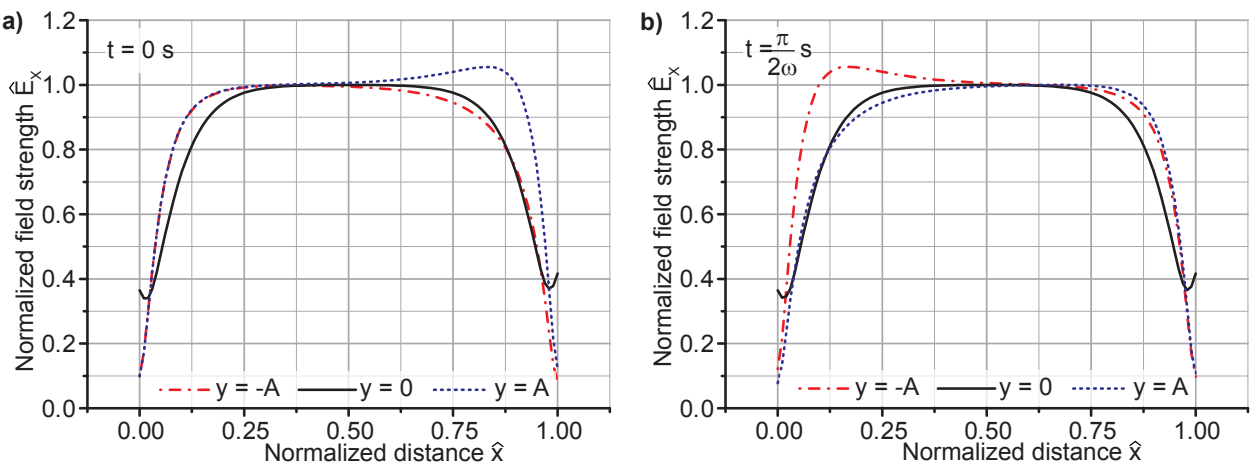

Figure 6. Electric field strength along the chamber for the zero entrance deflection (a) and the maximum entrance deflection (b). Plots for various transverse positions (y) are shown. The values are normalized by the electric field strength in the middle of the chamber, which equalled 0.965 of the desired separation field strength $E_{s}$. 


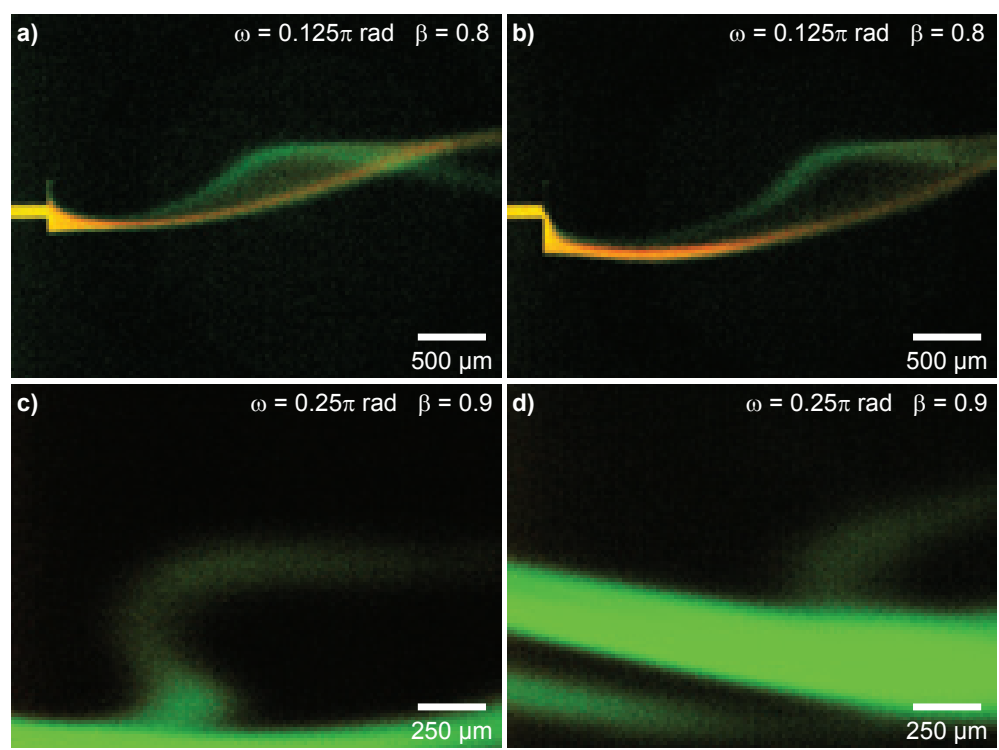

Figure 7. 'Strange' waves patterns observed during extreme modulation.

One approach that improves the field homogeneity in the electrokinetically operated microfluidic flow chambers is the addition of a number of parallel channels to the chamber structure. ${ }^{5-7}$ Those channels lay in the direction transverse to the flow direction and have their outlets positioned serially along the top and bottom walls of the chamber. Also introducing many parallel inlets and outlets seems to help. ${ }^{6}$ The second technique could be particularly interesting because putting many serially arranged sample inlets at the chamber entrance and applying a technique of synchronized sweeping of the active inlet - that is having only one of them introducing the sample stream for any instant, could possible reduce the effect observed during fast modulation of the wave amplitudes, when less mobile fractions produce waves with amplitudes smaller than predicted by theory. 


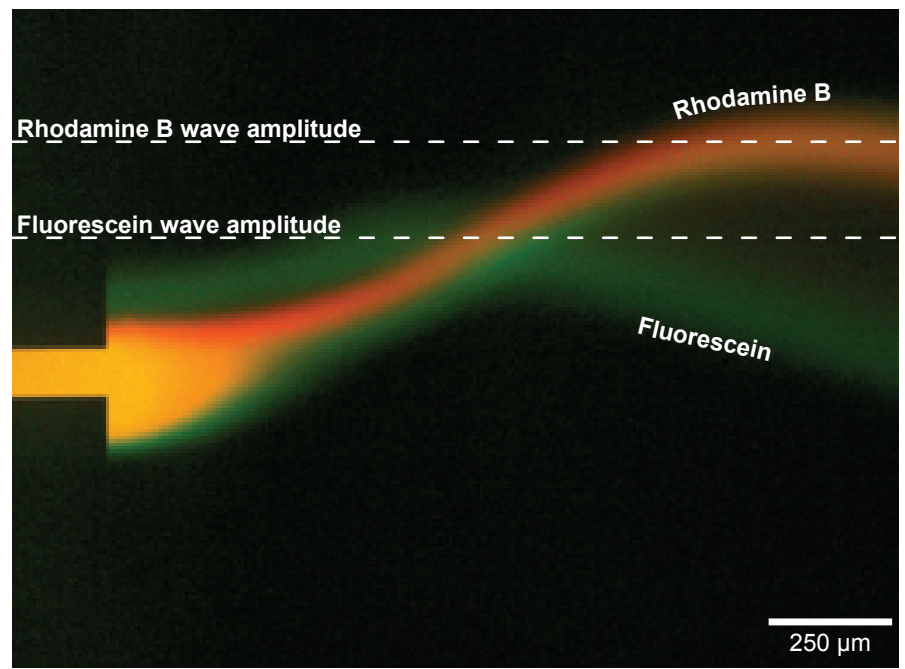

Figure 8. Different amplitudes of waves at the chamber entrance for fast amplitude modulation. Fluorescein is a less mobile fraction under the conditions used in the experiment.

\section{Outlook for SCFZE}

Even the performance of the current SCFZE device can be greatly improved by changing the synchronization schemes in such a way, that only half-period waves are injected. The starting stream position for which collection with a double-outlet SCFZE device is achievable can be easily calculated by using the theory introduced in this thesis. In such a scheme recovery of the injected material should at least double as compared to the currently used schemes.

The general idea of performing separation in the flow direction in a continuously operated device used in SCFZE has a great potential - not only for electrophoretic applications, where the clear benefit is the reduction of control hardware as compared to FFE. A similar technique could possibly also be applied to on-a-chip chromatography. The transition from SCFZE to continuous-flow electrochromatography seems straightforward.

\section{References}

1. Li, G., R. Ran, J.L. Zhao, and Y.S. Xu, Design, simulation, and optimization of a miniaturized device for size-fractioned DNA extraction. Electrophoresis, 2007. 28(24): p. 4661-4667. 
2. Sun, K., Z.Y. Li, K. Ueno, S. Juodkazis, S. Noji, and H. Misawa, Electrophoretic chip for high-fidelity fractionation of double-stranded DNA. Electrophoresis, 2007. 28(10): p. $1572-1578$.

3. Lin, R., D.T. Burke, and M.A. Burns, Selective extraction of size-fractioned DNA samples in microfabricated electrophoresis devices. Journal of Chromatography A, 2003. 1010(2): p. 255-268.

4. Lin, R., D.T. Burke, and M.A. Burns, Addressable Electric Fields for Size-Fractioned Sample Extraction in Microfluidic Devices. Analytical Chemistry, 2005. 77(14): p. $4338-4347$.

5. Estevez-Torres, A., C. Gosse, T. Le Saux, J.F. Allemand, V. Croquette, H. Berthoumieux, A. Lemarchand, and L. Jullien, Fourier analysis to measure diffusion coefficients and resolve mixtures on a continuous electrophoresis chip. Analytical Chemistry, 2007. 79(21): p. 8222-8231.

6. Lerch, M.A. and S.C. Jacobson, Electrokinetic fluid control in two-dimensional planar microfluidic devices. Analytical Chemistry, 2007. 79(19): p. 7485-7491.

7. Lerch, M.A., M.D. Hoffman, and S.C. Jacobson, Influence of channel position on sample confinement in two-dimensional planar microfluidic devices. Lab on a Chip, 2008. 8(2): p. 316-322. 



\section{Appendix A}

An example of an uExec script used for separation and accumulative fraction collection of a three-component mixture. The first and third components are pooled, the second component is discarded to the waste.

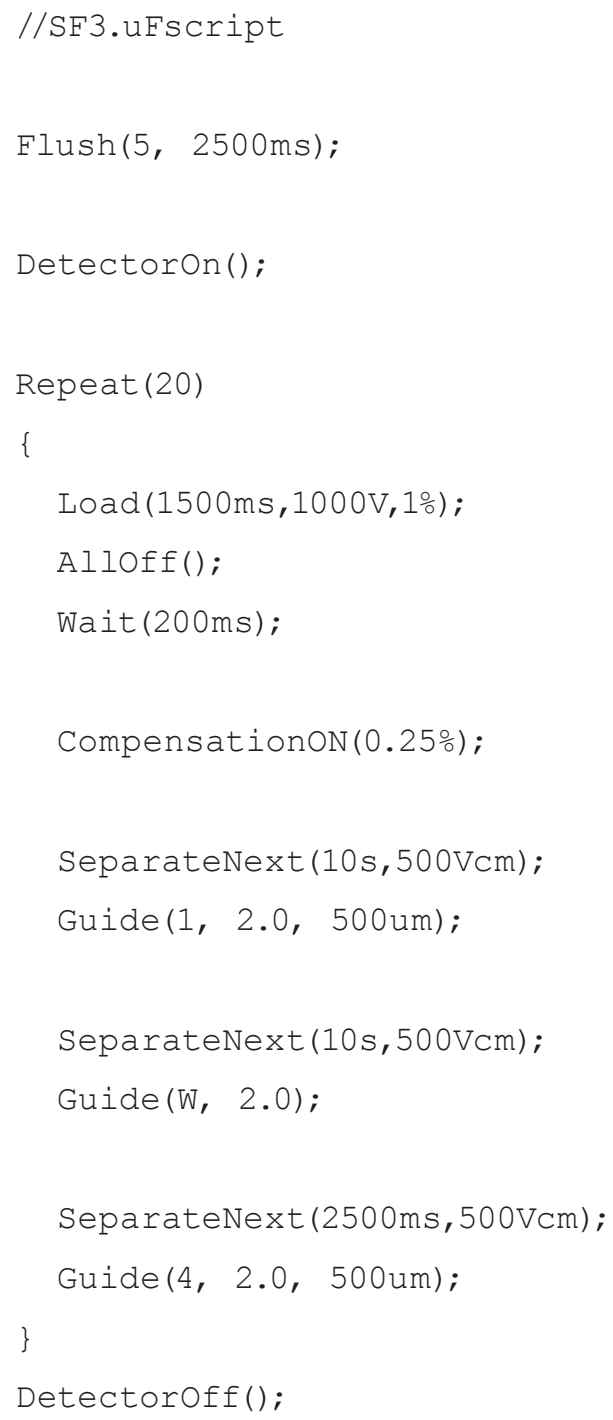





\section{Appendix B}

Matlab m-files for calculating performance measures of fractionation by SCFZE device described in chapter 6.

File 'SCFZE.m':

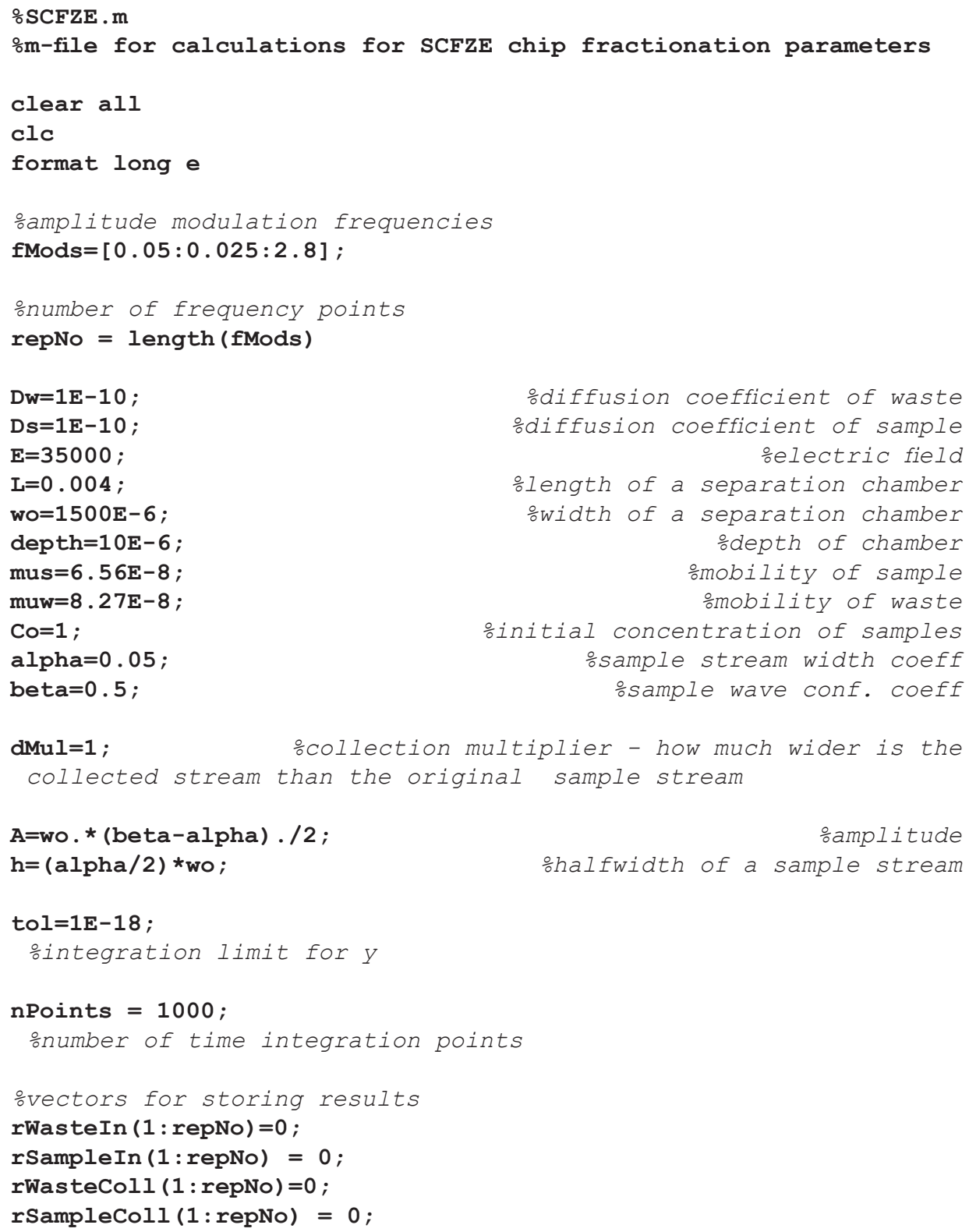


omain loop start

for repetition=1: repNo

$\mathrm{w}=2 \star \mathrm{pi}$ * $\mathrm{Mods}$ (repetition); periodTime $=1 . * 2 . * \mathrm{pi} . / \mathrm{w}$;

ts $=@(x) \quad x . /($ mus . *E); tw=@ (x) $x . /\left(\right.$ muw. $\left.{ }^{*} E\right)$; $y s=e(x, t)-A * \sin \left(w_{*}^{*}\left(x . / E_{0} / m_{u s}-t\right)\right) ;$ at the outlet

$\mathbf{y w}=@(\mathbf{x}, \mathbf{t})-\mathbf{A} .{ }^{*} \sin (\mathbf{w} . *(\mathbf{x} . / \mathbf{E} . / \mathrm{muw}-\mathrm{t}))$; $\frac{\circ}{0}$ waste stream position at the outlet

\section{oconcentration profile term for sample}

$\mathrm{Cs}=@(\mathrm{x}, \mathrm{y}, \mathrm{t})(\mathrm{Co} . / 2) . *(\operatorname{erf}((\mathrm{h}-\mathrm{y}+\mathrm{ys}(\mathrm{x}, \mathrm{t})) /$

$\left.\left.\left(2 .{ }^{*} \operatorname{sqrt}(D s . * t s(x))\right)\right)+\operatorname{erf}\left(\left(h+y-y_{s}(x, t)\right) /(2 . * \operatorname{sqrt}(D s . * t s(x)))\right)\right)$;

oconcentratio profile term for waste

$\mathrm{Cw}=@(\mathrm{x}, \mathrm{y}, \mathrm{t})(\mathrm{Co} . / 2) . *(\operatorname{erf}((\mathrm{h}-\mathrm{y}+\mathrm{yw}(\mathrm{x}, \mathrm{t})) /$

(2. * sqrt $\left.\left.\left(\mathrm{Dw} .{ }^{*} \operatorname{tw}(\mathrm{x})\right)\right)\right)+\operatorname{erf}\left(\left(\mathrm{h}+\mathrm{y}^{-}\right.\right.$

$\left.\left.\mathrm{yw}(\mathrm{x}, \mathrm{t})) /\left(2 .{ }^{*} \operatorname{sqrt}\left(\mathrm{Dw} .{ }^{*} \mathrm{tw}(\mathrm{x})\right)\right)\right)\right)$;

ocalculation of total streams entering the chamber during the ofull modulation period

rSampleIn (repetition) =mus . *E . *periodTime . *wo *alpha;

rWasteIn (repetition) =muw . *E . *periodTime . *wo . *alpha;

otime delta for manual integration

$\mathrm{dT}=($ periodTime -0$) . /$ nPoints;

ostart of integration for collection

SampleCollected $=0$;

WasteCollected $=0$;

sampleOut $=$ quad $(\Theta(y)$ Cs $(L, y, 0)$, ys $(L, 0)-h$, ys $(L, 0)+h$, tol) + quad(e(y) Cs (L,y,periodTime), ys (L, periodTime) $-\mathrm{h}$, ys (L, periodTime) th, tol);

wasteOut $=$ quad $(@(y) C w(L, y, 0), y s(L, 0)-h, y s(L, 0)+h$, tol) + quad(e (y) Cw (L, y, periodTime), ys (L, periodTime) $-\mathrm{h}$, ys (L, periodTime) th, tol) ;

for $i=1$ :nPoints-1

clc disp (['Repetition: ' num2str(repetition) '/'

num2str(repNo) ' timePoint: ' num2str(i) '/' num2str(nPoints)]) time $=0+i * d T$;

sampleOut $=$ sampleOut $+2 .{ }^{*}$ quad $(@(y)$ Cs ( $\mathrm{L}, \mathrm{Y}, \mathrm{time})$,

ys (L, time) $-h$, ys (L, time) $+h$, tol) ;

wasteOut $=$ wasteOut+2.*quad (@ (y) Cw (L, y, time),

ys ( $L$, time) $-h$, ys ( $L$, time) $+h$, tol) ;

end

ond of integration for collection 
ostoring the results of integration

rSampleColl (repetition) $=$ mus . * $. *(\mathrm{dT} . / 2) . *$ sampleOut. *depth;

rWasteColl (repetition) $=$ muw. ${ }^{\star} \mathrm{E} .{ }^{\star}(\mathrm{dT} . / 2) .{ }^{*}$ wasteOut. ${ }^{\star}$ depth

end

ond of main loop

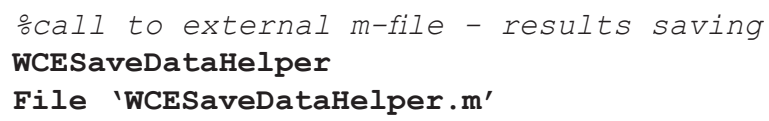


fprintf (fid,'Es - separation field / $\mathrm{V} / \mathrm{m} \backslash \mathrm{n}^{\prime}$ );

fprintf (fid,'LO - length of the chamber $/ \mathrm{m} \backslash \mathrm{n}^{\prime}$ );

fprintf (fid,'w0 - width of the chamber / $\mathrm{m} \backslash \mathrm{n}^{\prime}$ );

fprintf(fid,' alpha - sample stream width coeff $\backslash \mathrm{n}^{\prime}$ );

fprintf(fid,'beta - sample confinement coeff $\backslash \mathrm{n}^{\prime}$ );

fprintf(fid,'delta - sample stream diffusion coeff $\backslash \mathrm{n}^{\prime}$ );

fprintf(fid,'C0 - concentration of the analyte $\left.\backslash n^{\prime}\right)$;

fprintf (fid,'mus - mobility of the sample $1 / \mathrm{m}^{\wedge} 2 /(\mathrm{Vs}) \backslash \mathrm{n}^{\prime}$ );

fprintf (fid,'muw - mobility of the sample $2 / \mathrm{m}^{\wedge} 2 /(\mathrm{Vs}) \backslash \mathrm{n}^{\prime}$ );

fprintf (fid,'Ds - diffusion coefficient of sample $1 / \mathrm{m}^{\wedge} 2 / \mathrm{s} \backslash \mathrm{n}^{\prime}$ );

fprintf (fid,'Dw - diffusion coefficient of sample $2 / \mathrm{m}^{\wedge} 2 / \mathrm{s} \backslash \mathrm{n}^{\prime}$ );

fprintf(fid,' depth - depth of the chamber / $\left.m \backslash n \backslash n^{\prime}\right)$;

fclose (fid) ; 


\section{Appendix C}

Matlab m-files for calculating performance measures of fractionation by SCFZE device described in chapter 7.

\section{File 'DSCFZE.m'}

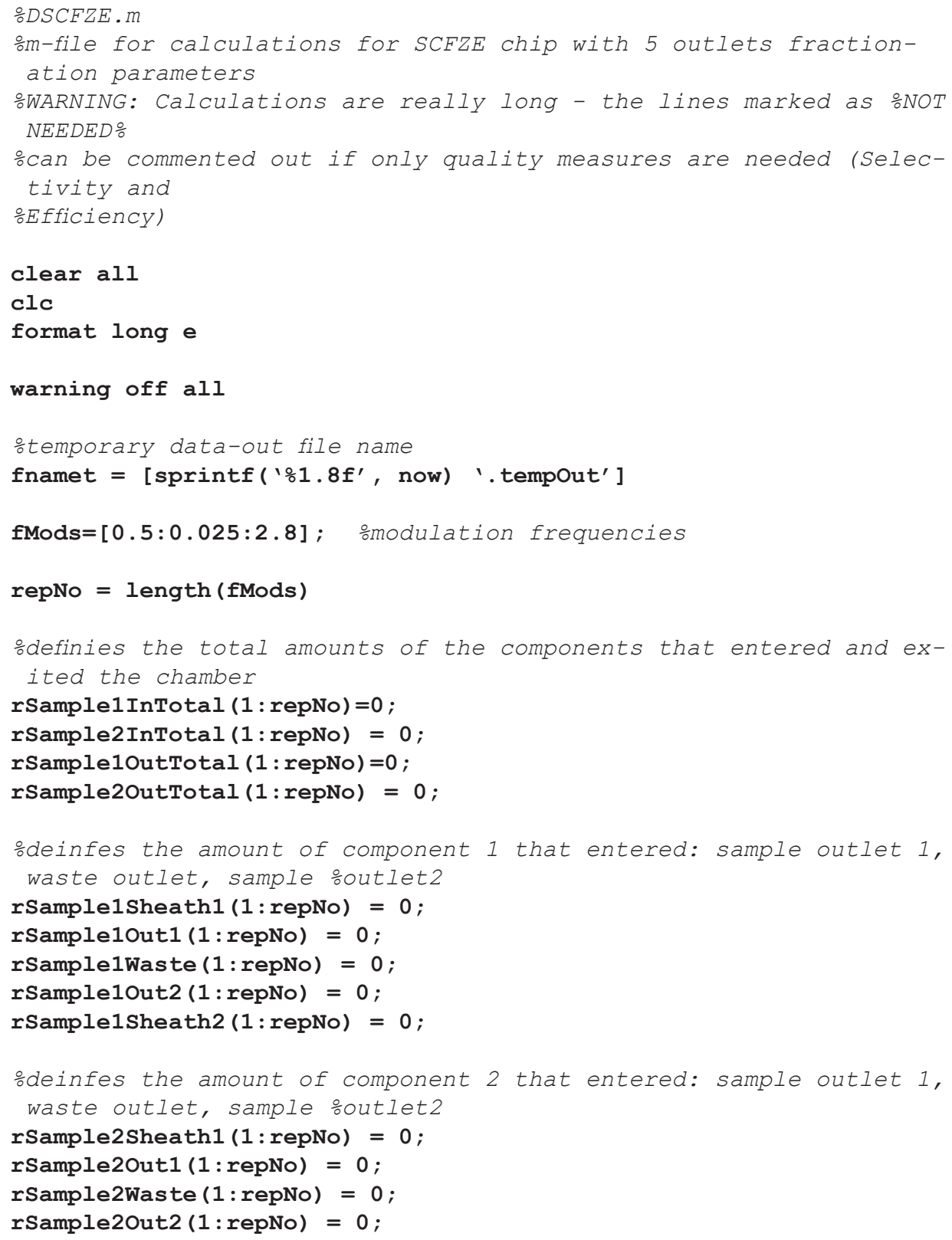


rSample2Sheath2 $(1:$ repNo $)=0$;

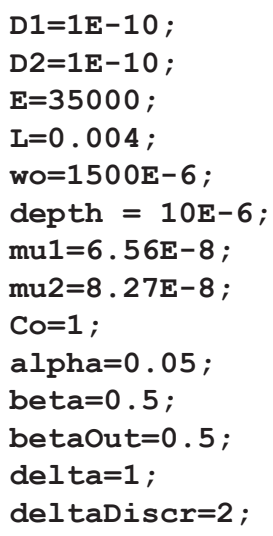

ohalfWidth of sample stream ohalfWidth of the sample stream for collection

ocall to external m-file for temporary data save

TemporarySaveBeginWrite;

omain loop start

for repetition=1 : repNo

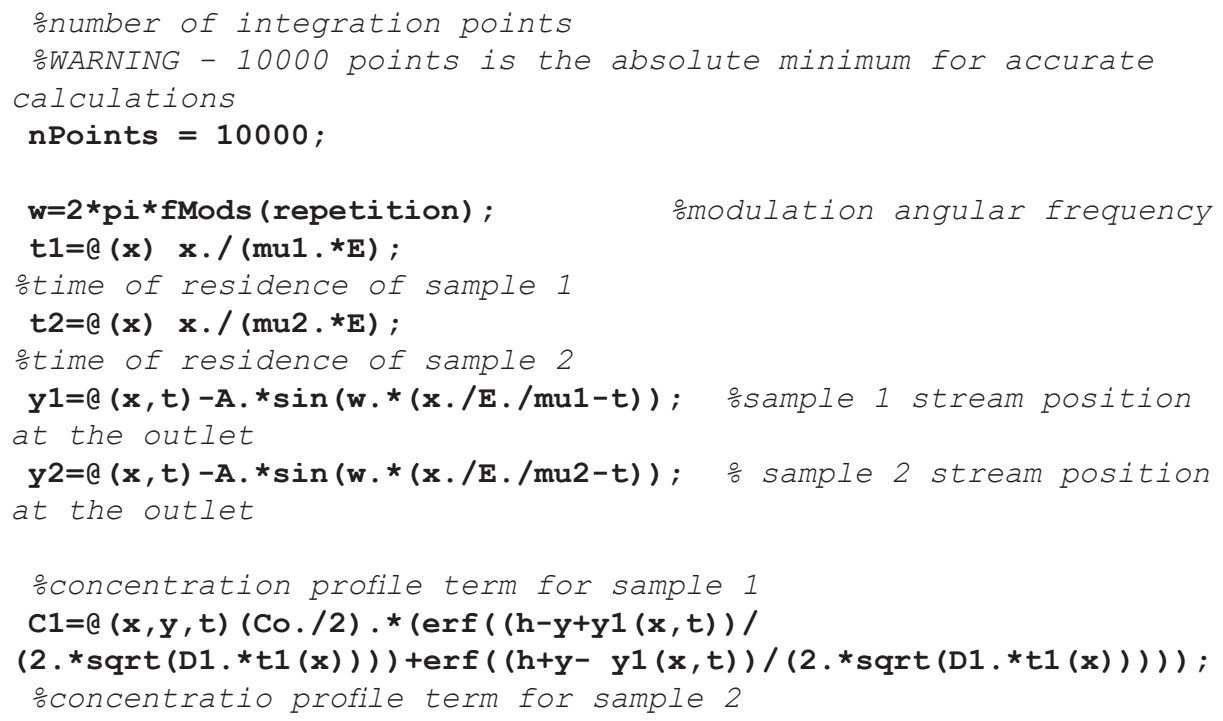


$\mathrm{C} 2=\mathrm{a}(\mathrm{x}, \mathrm{y}, \mathrm{t})(\mathrm{Co} \cdot / 2) \cdot *(\operatorname{erf}((\mathrm{h}-\mathrm{y}+\mathrm{y} 2(\mathrm{x}, \mathrm{t})) /$

$\left.\left.\left(2 .{ }^{*} \operatorname{sqrt}\left(\mathrm{D} 2 .{ }^{\star} t 2(\mathrm{x})\right)\right)\right)+\operatorname{erf}\left((\mathrm{h}+\mathrm{y}-\mathrm{y} 2(\mathrm{x}, \mathrm{t})) /\left(2 .{ }^{*} \operatorname{sqrt}(\mathrm{D} 2 . * t 2(\mathrm{x}))\right)\right)\right)$;

periodTime $=1 . * 2 . * \mathrm{pi} . / \mathrm{w}$;

ofull modulation period time

rSample1InTotal (repetition) =mu1 . *E . *periodTime . *wo . *alpha;

rSample2InTotal (repetition) =mu2 . *E . *periodTime . *wo . *alpha;

othere will be a total number of 10 integrals for each time

step!!! - keep

oit in mind

ohere they get defined:

Sample1Sheath $1=0$;

Sample10ut1= 0 ;

Sample1Waste $=0$;

Sample10ut2=0;

Sample1Sheath2=0;

Sample2Sheath1=0;

Sample2Out1=0;

Sample2Waste $=0$;

Sample2Out2 $=0$;

Sample2Sheath2=0;

\%end of definition

otime delta

$\mathrm{dT}=($ periodTime -0$) . /$ nPoints $;$

ofirst calculate integrals at the beginning of the period

if $\left((\mathrm{y} 1(\mathrm{~L}, 0)-\mathrm{y} 2(\mathrm{~L}, 0))>\right.$ deltaDiscr. $\left.{ }^{\star} \mathrm{h}\right)$

ocollectionPhase

NOT NEEDED:

Sample1Sheath1 = quad (@ (y) C1 $(\mathrm{L}, \mathrm{y}, 0), \mathrm{y} 1(\mathrm{~L}, 0)$ thout, woh, tol);

Sample10ut1 $=$ quad $\left(e(y) C 1(L, y, 0), y^{1}(\mathrm{~L}, 0)\right.$-hout, $\mathrm{y}^{1}(\mathrm{~L}, 0)$ thout, tol);

NOT_NEEDED

Sample1Waste $=$ quad $\left(@(y) C 1(L, y, 0), y 2(L, 0)\right.$ thout, $y^{1}(L, 0)-$ hout, tol);

Sample10ut2 $=$ quad $\left(\Theta(y) C 1(L, y, 0), y^{2}(L, 0)\right.$-hout, $\mathrm{y}^{2}(\mathrm{~L}, 0)$ thout, tol);

NOT_NEEDED:

Sample1Sheath2 = quad (@ (y) C1 (L,y,0), -woh, y2 (L, 0)-hout, tol);

NOT NEEDED :

Sample2Sheath1 = quad (@ (y) C2 $(L, y, 0), y 1(L, 0)$ thout, woh, tol);

Sample2Out1 $=$ quad $\left(\Theta(y) C 2(L, y, 0), y^{1}(L, 0)\right.$-hout, $y^{1}(L, 0)$ thout, tol);

\%NOT_NEEDED\%:

Sample2Waste $=$ quad $\left(@(y) C 2(L, y, 0), y^{2}(L, 0)\right.$ thout, $y^{1}(L, 0)-$ hout, tol);

Sample2Out2 $=$ quad $\left(\Theta(y) C 2(L, y, 0), y 2(L, 0)\right.$-hout, $y^{2}(L, 0)$ thout, 
tol);

NOT NEEDED:

Sample2Sheath2 = quad (@ (y) C2 $(\mathrm{L}, \mathrm{y}, 0)$, -woh, y2 (L, 0)-hout, tol);

else

oNon-Collection Phase

NOT NEEDED:

Sample1Sheath1 $=$ quad $(@(y) C 1(L, y, 0)$, betaOut. *wo./2, woh, tol) ;

○NOT NEEDED :

Sample1Waste $=$ quad $(\varrho(y) C 1(L, y, 0)$, -betaOut. *wo./2,

betaOut. *wo./2, tol);

NOT NEEDED:

Sample1Sheath2 $=$ quad $(@(y) C 1(L, y, 0),-$ woh, -betaOut. *wo./2, tol);

NOT NEEDED :

Sample2Sheath1 $=$ quad $(@(y) C 2(L, y, 0)$, betaOut. *wo./2, woh, tol);

NOT NEEDED:

Sample2Waste $=$ quad $(@(y) C 2(L, y, 0)$, -betaOut. *wo./2, betaOut. *wo./2, tol);

NOT NEEDED:

Sample2Sheath2 = quad(@ (y) C2 $(L, y, 0)$, -woh, -betaOut. *wo./2, tol);

end

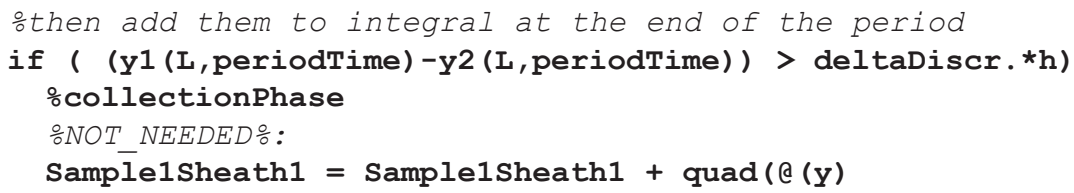




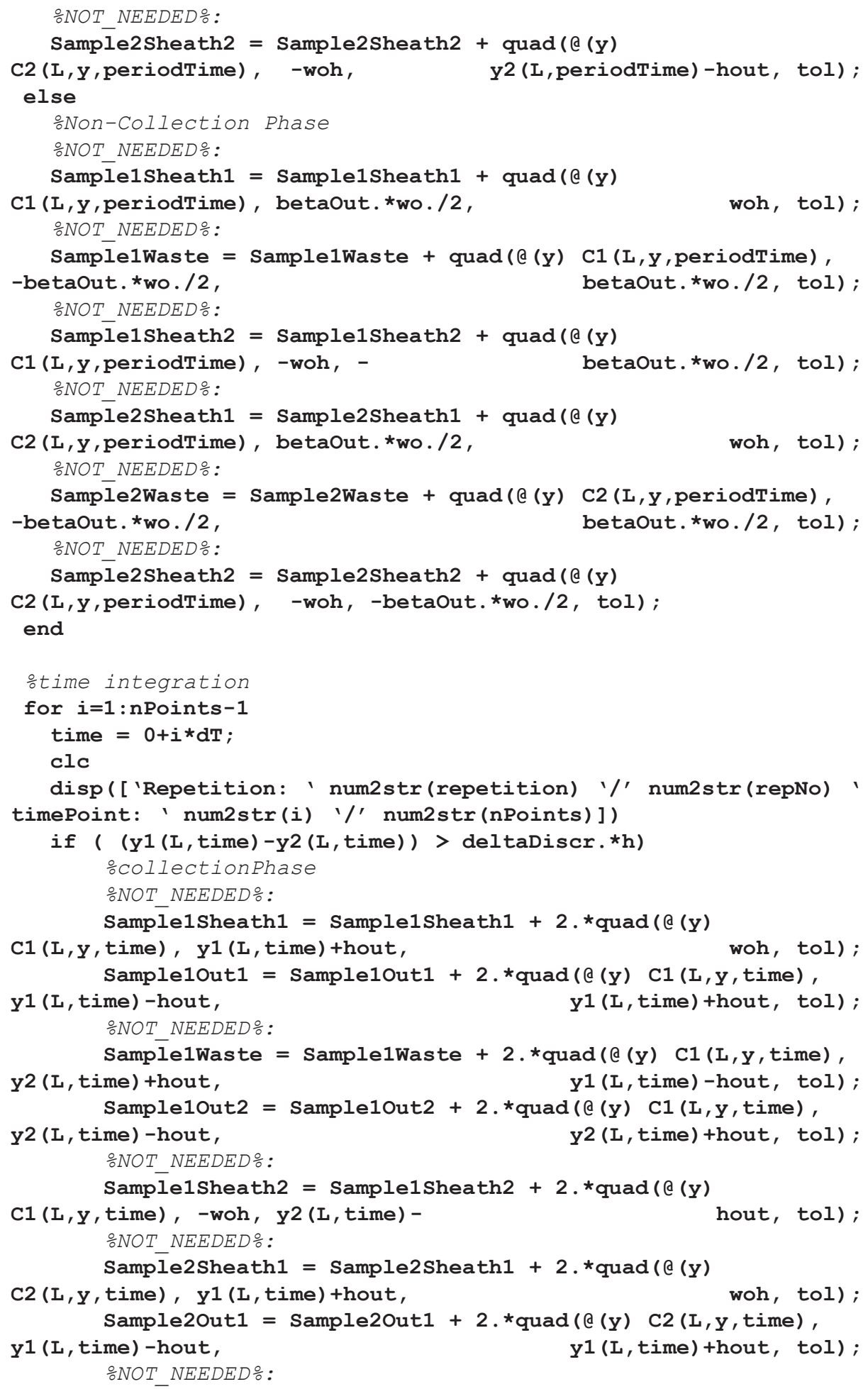




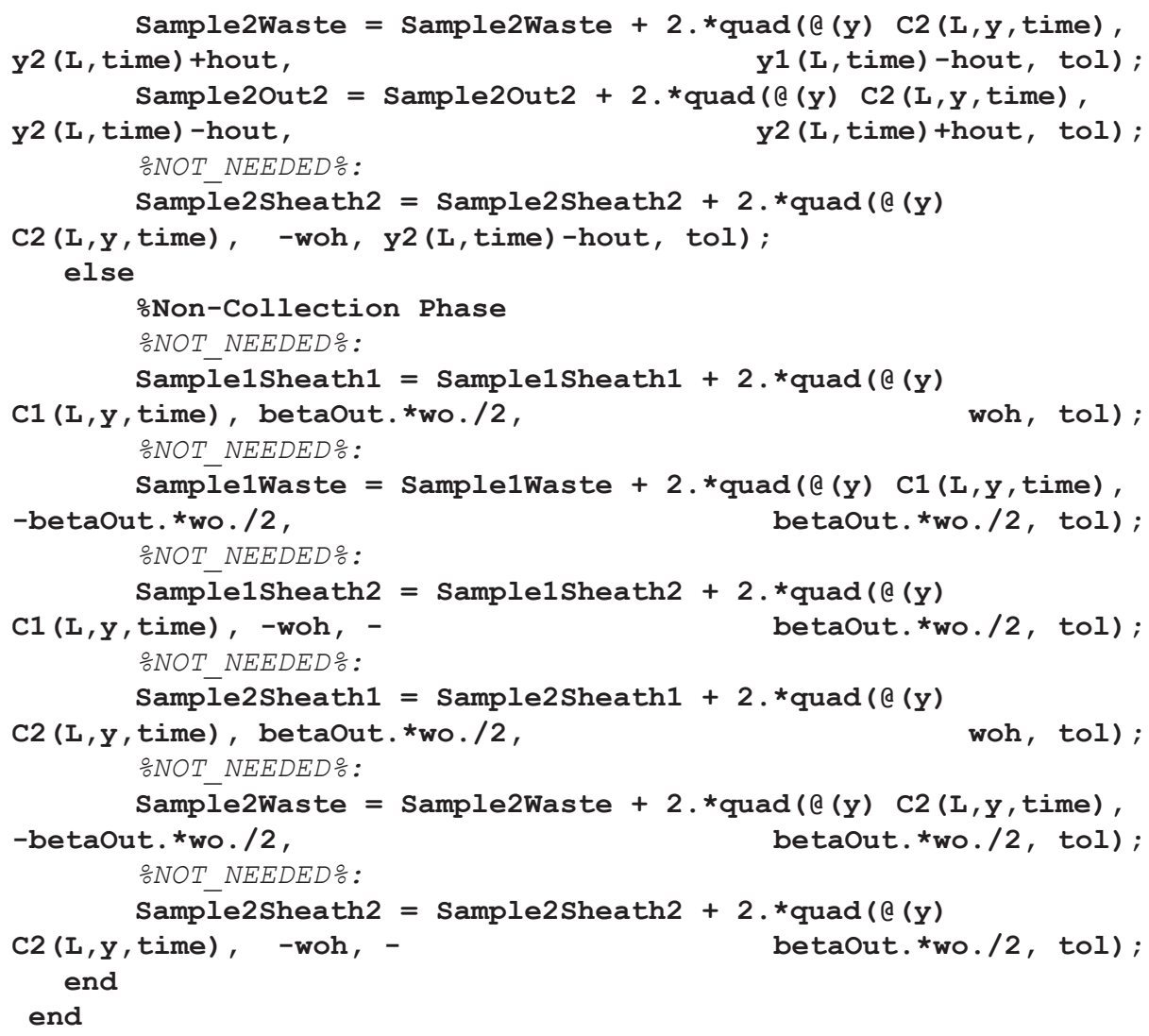


end

rSample1Sheath1 = depth.*rSample1Sheath1;

rSample10ut1 = depth. ${ }^{*}$ rSample10ut1;

rSample1Waste $=$ depth.${ }^{*}$ rSamplelWaste;

rSample10ut2 = depth. ${ }^{*}$ Sample10ut2;

rSample1Sheath2 = depth. ${ }^{*}$ Sample1Sheath2;

rSample2Sheath1 = depth. *rSample2Sheath1;

rSample2Out1 = depth. *rSample2Out1;

rSample2Waste $=$ depth.${ }^{*}$ rSample2Waste;

rSample2Out2 = depth. *rSample2Out2;

rSample2Sheath2 = depth. *rSample2Sheath2;

rSamplelInTotal $=$ depth.${ }^{*}$ rSamplelInTotal

rSampleloutTotal = depth. *rSampleloutTotal

rSample2InTotal = depth. *rSample2InTotal

rSample2OutTotal $=$ depth. ${ }^{*}$ rSample2OutTotal

ocall to external m-file for final data save

SaveDataHelper 
File 'TemporarySaveBeginWrite.m'

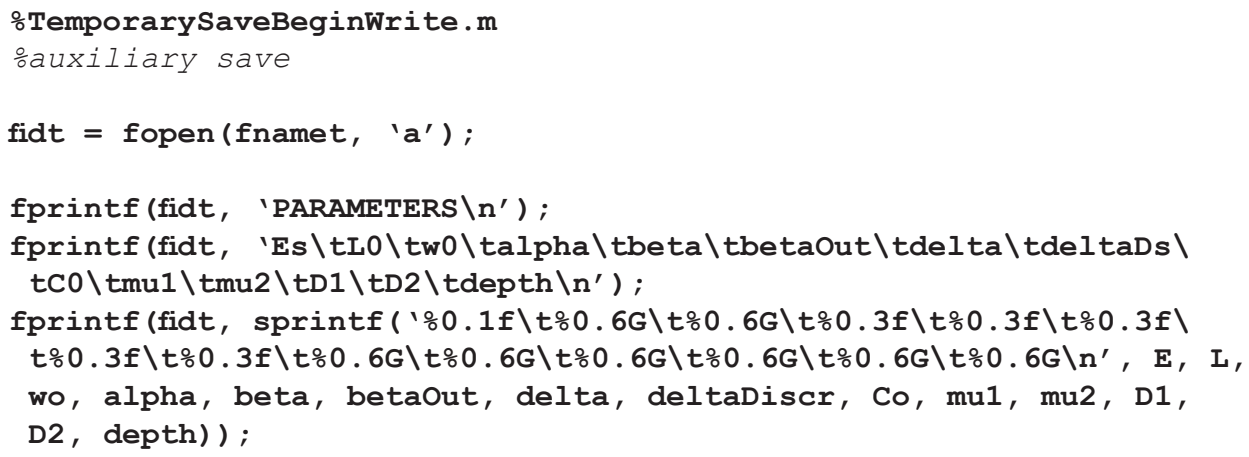

fprintf (fidt, ' $\backslash n \backslash n R E S U L T S \backslash n^{\prime}$ );

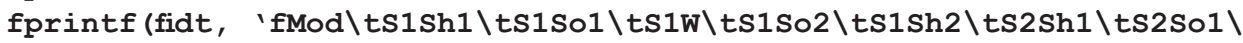
$t S 2 W \backslash t S 2 S o 2 \backslash t S 2 S h 2 \backslash t|\backslash t S 1 T I N \backslash t S 1 T O U T \backslash t S 2 T I N \backslash t S 2 T O U T \backslash t| \backslash t C 1 \backslash t C 2 \backslash$ $\left.t S 1 \backslash t S 2 \backslash n^{\prime}\right)$;

fclose (fidt)

ond of auxiliary save

File 'TemporarySave.m'

\%TemporarySave.m

oauxiliary save

fidt $=$ fopen $\left(\right.$ fnamet,$\left.{ }^{\prime} a '\right)$;

formatstringtt $=\backslash \div 3.6 \mathrm{G} \backslash t \div 3.6 \mathrm{E} \backslash t \div 3.6 \mathrm{E} \backslash t \div 3.6 \mathrm{E} \backslash t \div 3.6 \mathrm{E} \backslash t \div 3.6 \mathrm{E} \backslash t \div 3.6 \mathrm{E} \backslash$ $t \div 3.6 E \backslash t \div 3.6 E \backslash t \div 3.6 E \backslash t \div 3.6 E \backslash t|\backslash t \div 3.6 E \backslash t \div 3.6 E \backslash t \div 3.6 E \backslash t \div 3.6 E \backslash t| \backslash$ $t \div 3.6 E \backslash t \div 3.6 E \backslash t \div 3.6 E \backslash t \div 3.6 E \backslash n^{\prime}$;

tC1=rSample10ut1 (repetition). /rSample1 InTotal (repetition) ;

tC2=rSample2Out2 (repetition). /rSample2InTotal (repetition) ;

tS1=rSample1Out1 (repetition) . *rSample2InTotal (repetition) . /

rSample2Out1 (repetition). /rSample1InTotal (repetition);

tS2=rSample2Out2 (repetition) . *rSample1InTotal (repetition) . /

rSample10ut2 (repetition). /rSample2InTotal (repetition);

stringRowt $=$ sprintf (formatStringtt, fMods (repetition), depth.*rSa mple1Sheath1 (repetition), depth. *rSample10ut1(repetition), depth. ${ }^{*}$ rSample1Waste(repetition), depth. *rSample10ut2 (repetition), dept h. * ${ }^{*}$ Sample1Sheath2 (repetition), depth . *rSample2Sheath1 (repetitio n), depth.*rSample2Out1 (repetition), depth. *rSample2Waste (repetit ion), depth.*rSample2Out2 (repetition), depth. *rSample2Sheath2 (rep etition), depth.*rSamplelInTotal (repetition), depth. *rSample10utT otal (repetition), depth.*rSample2InTotal(repetition), depth.*rSam ple2OutTotal(repetition), tC1, tC2, tS1, tS2);

fprintf (fidt, stringRowt);

fclose (fidt)

ond of auxiliary save 


\section{File 'SaveDataHelper.m'}

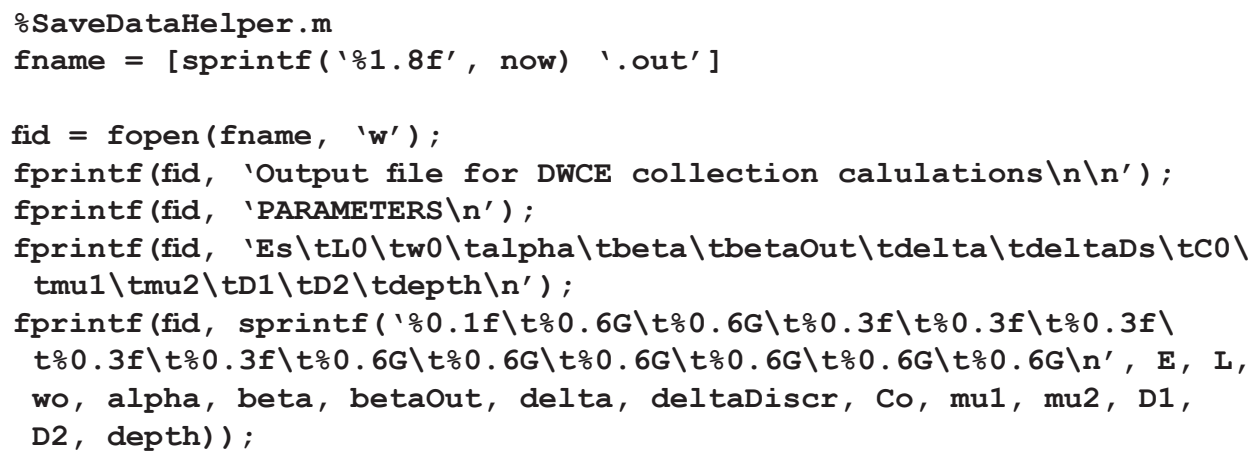


fprintf(fid,'S1Sh1 - Sample 1 collected in Sheath 1 reservoir $\backslash n^{\prime}$ ); fprintf(fid,'S1So1 - Sample 1 collected in Sample Outlet 1

reservoir $\left.\backslash n^{\prime}\right)$;

fprintf(fid,'S1W - Sample 1 collected in Waste reservoir $\backslash n^{\prime}$ );

fprintf(fid,'S1So2 - Sample 1 collected in Sample Outlet 2

reservoir $\left.\backslash \mathrm{n}^{\prime}\right)$;

fprintf(fid,'S1Sh2 - Sample 1 collected in Sheath 2 reservoir $\backslash \mathrm{n}^{\prime}$ );

fprintf(fid,'S2Sh1 - Sample 2 collected in Sheath 1 reservoir $\backslash \mathrm{n}^{\prime}$ );

fprintf(fid,'S2So1 - Sample 2 collected in Sample Outlet 1

reservoir $\left.\backslash n^{\prime}\right)$;

fprintf(fid,'S2W - Sample 2 collected in Waste reservoir $\backslash n^{\prime}$ );

fprintf(fid,'S2So2 - Sample 2 collected in Sample Outlet 2

reservoir $\left(\mathrm{n}^{\prime}\right)$;

fprintf(fid,'S2Sh2 - Sample 2 collected in Sheath 2 reservoir $\backslash \mathrm{n}^{\prime}$ );

fprintf (fid,'S1TIN - total Sample 1 which eneterd the chamber $\backslash \mathrm{n}^{\prime}$ ); fprintf(fid,'S1TOUT - total Sample 1 which left the chamber $\backslash n^{\prime}$ );

fprintf(fid,'S2TIN - total Sample 2 which eneterd the chamber $\backslash n^{\prime}$ ); fprintf(fid,'S2TOUT - total Sample 2 which left the chamber $\backslash n^{\prime}$ );

fprintf (fid,'Es - separation field / $\mathrm{V} / \mathrm{m} \backslash \mathrm{n}^{\prime}$ );

fprintf (fid,'LO - length of the chamber / $\left.m \backslash n^{\prime}\right)$;

fprintf (fid,'w0 - width of the chamber / $\left.m \backslash n^{\prime}\right)$;

fprintf(fid,' alpha - sample stream width coeff $\backslash n^{\prime}$ );

fprintf(fid,'beta - sample confinement coeff $\backslash \mathrm{n}^{\prime}$ );

fprintf (fid,'betaOut - waste Suck during NC phase coeff $\backslash n^{\prime}$ );

fprintf(fid,'delta - sample stream diffusion coeff $\backslash \mathrm{n}^{\prime}$ );

fprintf(fid,'deltaDs - sample stream diffusion coeff for

discrimination $\left.\backslash \mathrm{n}^{\prime}\right)$;

fprintf (fid,'C0 - concentration of the analyte $\left\langle\mathrm{n}^{\prime}\right)$;

fprintf (fid,'mu1 - mobility of the sample $1 / \mathrm{m}^{\wedge} 2 /(\mathrm{Vs}) \backslash \mathrm{n}^{\prime}$ );

fprintf (fid,'mu2 - mobility of the sample $\left.2 / \mathrm{m}^{\wedge} 2 /(\mathrm{Vs}) \backslash \mathrm{n}^{\prime}\right)$;

fprintf (fid,'D1 - diffusion coefficient of sample $1 / \mathrm{m}^{\wedge} 2 / s \backslash n^{\prime}$ );

fprintf (fid,'D2 - diffusion coefficient of sample $2 / \mathrm{m}^{\wedge} 2 / s \backslash \mathrm{n}^{\prime}$ );

fprintf(fid,' depth - depth of the chamber / $\mathrm{m} \backslash \mathrm{n} \backslash \mathrm{n}^{\prime}$ );

fprintf (fid,'CE1 - ratio of how much of sample 1 was collected to how much entered $\left.\backslash n^{\prime}\right)$;

fprintf(fid,'CE2 - ratio of how much of sample 2 was collected to how much entered $\left.\backslash n^{\prime}\right)$;

fprintf (fid,'S1 - Selectivity of collection of sample $1 \backslash \mathrm{n}^{\prime}$ );

fprintf(fid,'S2 - Selectivity of collection of sample $2 \backslash n^{\prime}$ );

fprintf(fid,' Pur1 - Purification ratio of sample $1 \backslash n^{\prime}$ );

fprintf(fid,'Pur2 - Purification ratio of sample $2 \backslash n^{\prime}$ );

fclose (fid) ; 


\section{Summary}

This thesis describes research on preparative capillary electrophoresis on a chip. The research started as an attempt to develop methods for efficient, post-separation sample manipulation and handling. Specifically, such methods should provide a way for either immobilizing separated components in individual compartments for in situ analysis or pooling of identical components in order to provide sufficient amount of a sample for further off-chip, lab-scale processing. One of the main requirements was the use of electrokinetic flow control only and lack of any kind of mechanical actuation.

The techniques for simple, post-separation single fraction handling are described in Chapter 3 of this thesis. Manipulation and arresting of fractions take place in a 2D laminar-flow bed. The sample is sandwiched between two sheath streams which are adjusted to control position and width of the sample stream. As a result separated fractions are guided one by one into different parallel lanes. The width of fractions during transfer is determined by the separation channel width and focusing rather than by injection volume and diffusion, by which cross-over between collection lanes can be avoided. The presented concept may be applied to deliver a separated sample to a secondary separation column, but also to enable in-situ measurements of separated fractions with optical techniques, where both considerable amount of sample and long measurements time are required.

The miniaturization of CE brings one important drawback: the resolution of separation is very limited as compared to traditional instruments. Consequently, separated fractions often overlap or are closely spaced at the end of a separation channel, making precise handling of individual peaks virtually impossible, which is also observed in experiments described in Chapter 3. Therefore in Chapter $4 \mathrm{a}$ method for forced electrokinetic splitting of adjacent fractions is proposed, which can be straightforwardly integrated into a micropreparative CE chip design. The method involves an extra T-junction at the end of a separation channel and detectortriggered reconfiguration of voltages at channel outlets. Forced splitting of a separated four-component mixture is demonstrated, and possible sources of errors leading to contamination of split fractions are also discussed in detail and illustrated both by computational fluid dynamics and experiments. The splitting method can be applied in preparative CE-on-a-chip systems, for which it greatly simplifies 
downstream fraction manipulation and helps in reducing cross-contamination between collected fractions.

The method of fractions splitting presented in Chapter 4 was applied in a micropreparative capillary electrophoresis chip described in details in Chapter 5. The device is capable of selecting and collecting multiple components from a separated mixture. This collection is automated and can be easily controlled by a set of rules defined by an operator, enabling fast and consistent operation. The device consists of an electrokinetically steered fluidic network that can be divided into three sections: a CE part, a fractions distribution region and a set of storage channels. Sample fractions leave the CE channel and are detected in the interfacial region by fluorescence intensity measurements. If an upcoming peak is detected, separation is withheld and the potentials are reconfigured to force the fraction into one of the collection channels, where they become available for further processing or analysis. The sequence of separation and collection is repeated until all the bands of interest are captured. Furthermore, it is possible to run the system in a repetitive mode for accumulative pooling if more fractionated sample is required.

Batch processing of a sample, as described in chapters 3-5 has one important drawback: the amount of an analyte obtained from a single run is very limited and collection of a sufficient amount is a time-consuming process. Therefore, in Chapter 6 a new method for performing continuous electrophoretic separation of complex mixtures in microscale devices, called continuous-flow zone electrophoresis (CFZE) is proposed. Unlike in free-flow electrophoresis devices, no mechanical pumping is required - both fluid transport and separation are driven electrokinetically. This gives the method a great potential for on-a-chip integration in multistep analytical systems. A preparative mode of the method, named synchronized, continuous-flow zone electrophoresis (SCFZE) enables to collect fractionated sample and tens-fold purification is possible. The model of the operation is presented and a detailed description of the optimal conditions for performing purification is given.

Chapter 7 describes a follow-up development of SCFZE described in Chapter 6. A solution is presented which allows for simultaneous collection of two fractions. Moreover, in contrast with the previous design, the collected samples are not crosscontaminated. The performance of the method is demonstrated on a model mixture and evaluated. by performing capillary electrophoresis of the collected fractions. Theoretical analysis shows that fractionation selectivity can be increased hundredsfold as compared to the earlier approach. 


\section{Samenvatting}

Dit proefschrift beschrijft onderzoek aan preparatieve capillaire elektroforese op een chip. Het onderzoek werd gestart met de bedoeling om methodes te ontwikkelen voor efficiënte monsterbehandeling na een analytische scheiding. Van deze methodes werd verwacht dat ze een manier zouden leveren voor ofwel het immobiliseren van gescheiden componenten in individuele compartimenten voor in situ analyse ofwel het ophopen van identieke componenten om daarmee voldoende monster te verkrijgen voor verdere verwerking buiten de chip, op laboratoriumschaal. Eén van de belangrijkste eisen was om uitsluitend gebruik te maken van gecontroleerde elektrokinetische stroming en geen enkele vorm van mechanische sturing.

Hoofdstuk 3 van dit proefschrift beschrijft technieken voor een eenvoudige behandeling van geïsoleerde fracties na hun scheiding. Manipulatie en vasthouden van fracties vindt plaats in een 2D laminaire-stromingskamer. Het monster wordt ingeklemd tussen twee beschermstromen die zodanig worden ingesteld dat de positie en de breedte van de monsterstroom zijn vastgelegd, met als resultaat dat de gescheiden fracties één voor één in afzonderlijke parallelle kanalen kunnen worden geleid. De breedte van de fracties gedurende dit proces wordt bepaald door de breedte van het scheidingskanaal en de focusseerprecisie en niet zozeer door injectievolume of diffusie. Hierdoor kan kruiselingse overdracht tussen de verzamelkanalen worden voorkomen. Het gepresenteerde concept kan worden toegepast om een gescheiden monster aan een tweede scheidingskolom over te dragen, maar ook om in-situ metingen te doen aan de gescheiden fracties met behulp van optische methodes, waarvoor zowel een aanzienlijke hoeveelheid monster als een lange meettijd vereist zijn.

Het miniaturiseren van CE heeft één belangrijk nadeel: de scheidingsresolutie is zeer beperkt in vergelijking met traditionele instrumenten. Dientengevolge overlappen gescheiden fracties vaak, of liggen ze aan het einde van de scheidingskolom te dicht bij elkaar om een nauwkeurige behandeling van individuele zones toe te laten. Dit laatste wordt ook waargenomen in de experimenten beschreven in Hoofdstuk 3. Om hierin verbetering te brengen is in Hoofdstuk 4 gekozen voor het geforceerd elektrokinetisch splitsen van naburige fracties, een methode die rechttoe-rechtaan kan worden geïntegreerd in een micropreparatief CE chip ontwerp. De methode behelst introductie van een extra T-junctie aan het einde van een scheidingskanaal en door een detector geïnitieerde reconfiguratie 
van de voltages op de kanaaluitgangen. Geforceerde splitsing van een gescheiden viercomponentenmengsel wordt gedemonstreerd, en mogelijke foutenbronnen die aanleiding geven tot verontreiniging van gesplitste fracties worden geïdentificeerd en zowel met computational fluid dynamics als met experimenten geïllustreerd. De splitsingsmethode kan worden toegepast voor preparatieve CE-op-een-chip, waarbij de stroomafwaartse fractiemanipulatie sterk wordt vereenvoudigd, en wederzijdse verontreiniging van verzamelde fracties sterk wordt verminderd.

De methode van fractiesplitsing uit Hoofdstuk 4 wordt gebruikt in een micropreparatieve capillaire elektroforese-chip in Hoofdstuk 5. Dit apparaat kan meerdere componenten uit een gescheiden mengsel selecteren en individueel opslaan. Het opslaan is geautomatiseerd en kan eenvoudig worden gestuurd via een aantal regels die door een operator kunnen worden gedefinieerd, waardoor een snelle en consistente werking kan worden gegarandeerd, Het apparaat bestaat uit een elektrokinetisch gestuurd vloeistofnetwerk dat drie sectoren heeft: een CE-gedeelte, een gebied waarin de fracties gedistribueerd worden, en een aantal opslagkanalen. Monsterfracties verlaten het CE-kanaal en worden met behulp van fluorescentie gedetecteerd in een overgangsgebied. Als een naderende zone wordt gedetecteerd, wordt de scheiding stopgezet en worden de potentialen gereconfigureerd om de fractie in één van de collectiekanalen te dirigeren, alwaar ze beschikbaar komen voor verdere verwerking of analyse. Deze sequentie van scheiding en collectie wordt herhaald totdat alle gewenste zones zijn opgevangen. Het is mogelijk om het systeem zodanig in te stellen dat de gehele loop zoals hiervoor beschreven herhaald blijft totdat voldoende van één of meerdere gewenste fracties is verzameld.

Partijgewijze ("batch") verwerking van een monster zoals beschreven in Hoofdstukken 3-5 heeft als belangrijkste nadeel dat de hoeveelheid stof die in één enkele cyclus wordt verkregen toch zeer beperkt is, en opvangen van voldoende van die stof via repeterende cycli een tijdrovend proces is. $0 \mathrm{~m}$ dit nadeel te ondervangen is in Hoofdstuk 6 een nieuwe methode voorgesteld om continue scheiding van complexe mengsels in miniatuurinstrumenten te bewerkstelligen, genaamd continue-stromings-zone-elektroforese (CFZE). Anders dan in vrije-stromings elektroforese, is hiervoor geen mechanische pomp vereist, zowel de vloeistof als de scheiding zijn elektrokinetisch gedreven. Hierdoor heeft de methode groot potentieel voor integratie in meertraps-analytische chipsystemen. Een preparatieve vorm van de methode, genaamd gesynchroniseerde continue-stromings zone elektroforese (SCFZE), bewerkstelligt de verzameling van gefractioneerd monster met een tienvoudige verhoging van de concentratie. Een model voor de werking 
hiervan wordt gepresenteerd en een gedetailleerde beschrijving van de optimale condities voor fractiezuivering wordt gegeven.

Hoofdstuk 7 beschrijft de doorontwikkeling van de SCFZE-techniek uit Hoofdstuk 6 tot een versie die de gelijktijdige collectie van twee fracties mogelijk maakt. In deze versie zijn, in tegenstelling tot het eerdere ontwerp, de verzamelde fracties niet verontreinigd. De werking van de methode wordt gedemonstreerd aan de hand van een modelmengsel en geëvalueerd via hernieuwde capillaire elektroforese van de verzamelde fracties. Een theoretische analyse toont aan dat de selectiviteit van het fractioneren honderdvoudig kan worden verhoogd in vergelijking met de eerdere aanpak. 



\section{Acknowledgements}

When you come to science and are given your first challenge to fight, there's usually a plentiful of, what you then think, brilliant, breakthrough ideas born in your mind - some of them surely worth the Nobel prize. As the time goes by, you learn that most of solutions to the problems already exist and the only thing you need to do is to spot and fish the right one. Surely, fishing is more enjoyable experience in a good company and it goes without saying that when you're after some really nippy fish a helpful hand is most needed and appreciated. Putting things this way, research is not much different from fishing - you just can't do it alone. And occasionally, when after weeks of fruitless tries, you get nothing but an old wellie and a rusty bike wheel and decide to abandon this whole fishing and turn to a more thrilling activity like ant-counting, on your way home you are lucky enough to meet a fisherman. With an expression showing a great shock he exclaims-'What were you doing over there with those sticks, you foolish boy, I ain't seen no fish for years in this lagoon'-and he invites you to follow him to his boat and presents you with the most fabulous fish you've ever seen. Well, that's what I think doing science looks like. Let me start with the fishermen I've met.

I wish to express my gratitude to Richard Schasfoort, who invited me to become a member of the Biochip Group and was my supervisor for nearly three years. Richard, your enthusiasm for science is highly contagious and I certainly picked up some of it. You also taught me to keep my mind open, as even what seems to be a most impossible or worthless idea can turn into gold. The friendliness, highly abundant in the group you created certainly made my moving to the Netherlands a soft landing.

It is beyond any doubt that this thesis would be completely different (if it existed) without the fantastic support I received from my second supervisor and promotor Han Gardeniers who, despite the depths of despair over my PhD project I was just in, took me into the Mesoscale Chemical Systems group. Han, I owe you a great deal, not only in a blink of an eye you taught me the importance of small discoveries - you also showed me that there is pride and enjoyment linked to them. I also highly value your understanding for my sometimes somewhat mulish way of working and the great patience you showed when I was writing this thesis and was always 'a bit late'. You keep surprising me with the little pieces of knowledge you come up with during our discussions, I wish one day I could learn even half of what you know. 
There is one person who helped me at a very demanding moment. During my transition from the Biochip group to the MCS Vinod Subramaniam and his group were marvellous hosts. Vinod, I remember you once told me not to worry, you were right.

I wouldn't have started in research without a person who taught me the first steps of the dance of science. Jan Dziuban, a supervisor of my master thesis at the Wroclaw University of Technology showed me that only hard work leads to success. He also pushed me to do a short student internship at the University of Twente, of which existence I was not aware then.

As I mentioned in the beginning, performing science in a good company is a cheerful experience, and assistance is most appreciated. It is my great belief that without the help of Stefan Schlautmann the technology, employed for the production of the chips I used, would have been at the level of development of the Stone Age - in the best case of course. I don't know how it happens, but when there's more than one beaker around me, something usually goes wrong, and cleanroom is exceptionally full of beakers, not to mention other equipment, the count of which goes beyond my understanding. Stefan, thanks a lot for all the hours you spent in this sanctuary of breaking glassware making the devices for me. I appreciate it a lot, especially knowing from experience how boring it is to sit there alone and watch deposition going at the tremendous speed of $\mathrm{nm} / \mathrm{min}$. There is also a lot to say about all the coffee breaks during which we sipped a 10th cup together (i.e. mine second and yours eighth) and all the evenings we spent trying to figure out the rate of bubble formation in a pint of Guinness. I think the best way I could express myself here is by saying: thanx a lot dude.

Hans de Boer is the most skilful magician I've ever met. I don't know the spells he used to transfer my curvy sketches into precise and ingenious instruments but I'm sure the power of them would knock out any skilled wizard. Hans, I'm really grateful for your support - you are also a most pleasant chap, you never even hinted your disapproval when a machine you'd made for me was used only once and dumped afterwards.

I already mentioned my violent behaviour when it comes to liquid matter enclosed in glass containers. Dietrich Kohlheyer certainly showed me a way to reduce my demolition powers in a lab. I could also always call him and ask for help when this dumb, thick-headed microscope just wouldn't read my thoughts properly. Didi, the 
chats we had during breaks and after work were always entertaining, I hope there are still unrevealed levels of humour, waiting for us out there.

I think I wouldn't have produced half of the results without a few little hints from Geert Besselink. Geert, I think you don't even remember those, but since my understanding of buffer chemistry is truly hopeless, if you hadn't tipped me off I would still be trying to get a decent separation with a sample dissolved in pure DI water.

There are a couple of extraordinary fellows who did they best to keep me in one piece when I was about to blow up and who were always there to cheer me up or just to gossip.

Wojtek is not a person of easy being and neither am I. Yet, astonishingly we are friends and during all this time he would always pick up my calls or go with me for a walk or just sit on a sofa and listen to what I had to say and then he would say what he thought about it. We shared all the frustrations of young researchers and all the fun that comes after it. Wojtek, thanks for all these years.

Bianca is somebody I can always drop by and chat a little about unimportant things as well as most serious matters. She would always listen patiently and talk back twice as much. Bianca, thank you for the optimism you share so generously.

Tomek was the lad I confided in most during my stay in the BPE and he was always extremely accommodating and always had time for me. Tomek, I'm really thankful for all the talks we had and occasionally still have.

The time I spent on my PhD couldn't be more enjoyable. And this is mostly thanks to wonderful people I had the pleasure to work and have fun with. Besides those I already mentioned I would like to thank all the members of the old Biochip group for all the crazy things we did together: Arnoud, Sandeep, Björn, Verena, Remco, Ada, Ganesh and others - thanks for all folks.

I am definitely a shy-monk type of person, yet my landing in the MSC group couldn't go with less effort I believe - that was mainly thanks to a smashing atmosphere that must be attributed to all the members of the MSC: David (my always laughing roommate), Maciek, Roald, Vincent, Jacob, Jacqueline, Nikolay, Regina, Selm, Wim, Eliza, Kevin, Anil, Manon, Sertan - I really owe you guys.

I would also like to thank the members of my new group, Cooling and Instrumentation in the Low Temperature Division, and especially my boss 
Marcel ter Brake for the patience I was treated with in the first two month of my post-doc, when I was still quite often occupied with the PhD-related tasks instead of spending time on the project.

Last but not least I would like to express my gratitude to my family. They were always there supporting me and pushing my ambitions higher. Mamo, tato dziękuję za wszystkie te lata, począwszy od pierwszej szkoły aż do teraz, kiedy mnie wspieraliście. Mamo, zawsze będę pamiętał czas, który spędziłaś na uczeniu mnie matematyki, wtedy nie znosiłem tych lekcji, dziś jestem dumny z tego, że każdy wzór rozumiem gdy tylko nań spojrzę. Tato, odkąd pamiętam namawiałeś mnie do uczenia się języków, byłeś moim pierwszym nauczycielem angielskiego, a potem utwierdzałeś mnie w przekonaniu że wyjazd za granicę to świetna decyzja. Jestem Wam za to bardzo wdzięczny.

My sister, Kaja, is now a charming young lady, as she would be called some hundred years ago. When we are together we always laugh a lot, that is, she laughs a lot and I try to keep up with her. Kaja, you are always a great joy and I easily forget about the burden of life when I talk with you.

The last and the most important paragraph of this thesis goes to my love and wife Kasia. We've spent so many years together I'm surprised every time I discover a new thing about her. Kasia, you've been always by my side - supporting me, cheering me up, having fun together or just being. I truly thank you for all the things you gave me - you are my sunshine on a gloomy day. 